\title{
Comparing tailored video- and text-based messages about smoking cessation in different socioeconomic groups : reach, adherence and (cost-) effectiveness
}

Citation for published version (APA):

Stanczyk, N. E. (2014). Comparing tailored video- and text-based messages about smoking cessation in different socioeconomic groups : reach, adherence and (cost-) effectiveness. [Doctoral Thesis, Maastricht University]. Maastricht University. https://doi.org/10.26481/dis.20141010ns

Document status and date:

Published: 01/01/2014

DOI:

10.26481/dis.20141010ns

Document Version:

Publisher's PDF, also known as Version of record

Please check the document version of this publication:

- A submitted manuscript is the version of the article upon submission and before peer-review. There can be important differences between the submitted version and the official published version of record.

People interested in the research are advised to contact the author for the final version of the publication, or visit the DOI to the publisher's website.

- The final author version and the galley proof are versions of the publication after peer review.

- The final published version features the final layout of the paper including the volume, issue and page numbers.

Link to publication

\footnotetext{
General rights rights.

- You may freely distribute the URL identifying the publication in the public portal. please follow below link for the End User Agreement:

www.umlib.nl/taverne-license

Take down policy

If you believe that this document breaches copyright please contact us at:

repository@maastrichtuniversity.nl

providing details and we will investigate your claim.
}

Copyright and moral rights for the publications made accessible in the public portal are retained by the authors and/or other copyright owners and it is a condition of accessing publications that users recognise and abide by the legal requirements associated with these

- Users may download and print one copy of any publication from the public portal for the purpose of private study or research.

- You may not further distribute the material or use it for any profit-making activity or commercial gain

If the publication is distributed under the terms of Article $25 \mathrm{fa}$ of the Dutch Copyright Act, indicated by the "Taverne" license above, 
Comparing tailored video- and text-based messages about smoking cessation in different socioeconomic groups:

Reach, adherence and (cost) effectiveness

Nicola Esther Stanczyk 


\section{COLOPHON}

Cover design and lay-out: Kikisoso creative studio | Nicola Esther Stanczyk

Production: Ridderprint BV, Ridderkerk, The Netherlands

(C) Nicola Esther Stanczyk, 2014

ISBN: 978-90-5335-909-9

The research presented in this thesis was conducted at the School for Public Health and Primary Care (CAPHRI), Department of Health Promotion, Maastricht University. CAPHRI participates in the Netherlands School of Primary Care Research (CaRe). CAPHRI was classified as "excellent" by the external evaluation committee of leading international experts that reviewed CAPHRI in December 2010. The research presented in this dissertation was funded by the Netherlands Organisation for Health Research and Development (ZonMW, Grant no. 20011007).

All rights are reserved. No part of this book may be reproduced or transmitted in any form or by any means, without the written permission from the author or, where appropriate, the publisher of the article. 
Comparing tailored video- and text-based messages about smoking cessation in different socioeconomic groups: Reach, adherence and (cost) effectiveness

\section{PROEFSCHRIFT}

ter verkrijging van de graad van doctor

aan de Universiteit Maastricht,

op gezag van de Rector Magnificus,

Prof. dr. L. L. G. Soete,

volgens het besluit van het College van Decanen,

in het openbaar te verdedigen

op vrijdag 10 oktober 2014 om 14:00 uur

door

Nicola Esther Stanczyk

geboren op 12 november 1986 te Keulen (Duitsland) 


\section{PROMOTOR}

Prof. dr. Hein de Vries

\section{COPROMOTOREN}

Dr. Catherine A. W. Bolman, Open Universiteit

Dr. Jean W. M. Muris

\section{BEOORDELINGSCOMMISSIE}

Prof. dr. Nanne K. de Vries (voorzitter)

Prof. dr. Rutger C. M. E. Engels, Radboud Universiteit Nijmegen

Prof. dr. Gerjo Kok

Prof. dr. Lilian Lechner, Open Universiteit

Prof. dr. Constant P. van Schayck 


\section{CHAPTER 1}

General introduction

\section{CHAPTER 2}

Study protocol of a Dutch smoking cessation e-health program

\section{CHAPTER 3}

How to encourage smokers to participate in web-based computer-tailored smoking cessation programs: a comparison of different recruitment strategies

\section{CHAPTER 4}

Influence of delivery strategy on message processing mechanisms and

future adherence to a Dutch computer-tailored smoking cessation intervention

\section{CHAPTER 5}

Comparison of text and video computer-tailored interventions for for smoking cessation: Randomized controlled trial

\section{CHAPTER 6}

Effectieveness of a video- and text-based computer-tailored smoking cessation intervention among LSES and HSES smokers

\section{CHAPTER 7}

An economic evaluation of a video- and text-based computer-tailored intervention for smoking cessation: a cost-effectiveness and cost-utility analysis of a randomized controlled trial

\section{CHAPTER 8}

General discussion 



\section{CHAPTER 1}

General introduction 



\section{Inequalities in smoking behaviour}

Smoking tobacco is one of the most preventable causes of illness and death (World Health Organization, 2008) and continues to be a major public health concern all over the world. Smoking is associated with an increased risk of different diseases, such as cardiovascular diseases (CVD), chronic obstructive pulmonary disease (COPD), asthmatic diseases and different types of cancer. Latest estimates from the World Health Organization (WHO) have suggested that about six million people worldwide die each year from smoking tobacco; approximately five million are smokers and ex-smokers and more than 600,000 are nonsmokers who were exposed to second-hand smoke (World Health Organization, 2011). Around two million people die each year from tobacco-induced CVD, followed by COPD and lung cancer which causes about one million deaths per year (Ezzati and Lopez, 2003, World Health Organization, 2008). Despite the negative effects of smoking, more than one billion people in the world remain smokers (World Health Organization, 2011). In the Netherlands, approximately 20,000 people die each year due to various smoking-related illnesses (Stivoro, 2011a). To date, Dutch smoking prevalence rates are still around $23 \%$ for women and $27 \%$ for men (Stivoro, 2011b).

Although smoking increases the risk of premature death among all consumers, the problem of tobacco use is greatest in groups of a lower socioeconomic status (LSES) (Giskes et al., 2005, Honjo et al., 2006, Kotz and West, 2009). Socioeconomic status (SES) can be defined as an individual's economic and social position, which is often based on several variables such as income, education and occupational status (Laaksonen et al., 2005). Disparities in SES related to smoking behaviour have become a progressively more serious problem over the last decades. Trends in SES related to inequalities in smoking over time have been described in detail in 1994 in the Smoking Epidemic Model (SEM) (Lopez et al., 1994). From an historical perspective, smoking tobacco first became socially accepted among people of a higher socioeconomic status (HSES) and then gradually increased among all population groups (Lopez et al., 1994). After some time, health campaigns on the hazards of cigarettes caused a decline in smoking prevalence rates especially among people of HSES, subsequently widening SES differences in the population. In most western countries, where smoking has reached the last stage of the SEM, socioeconomically disadvantaged people often smoke more than people with a higher HSES (Giskes et al., 2005, Harper and Lynch, 2007, Mackenbach et al., 2008, Rasmussen et al., 2009, Smith et al., 2009). During the last several years, SES inequalities in smoking prevalence have also increased in the Netherlands. Between the years 1988 and 2010, Dutch smoking prevalence rates declined rapidly within the more highly educated population, at $9 \%$, compared with people of middle and lower levels of education ( $2 \%$ and $5 \%$, respectively) (Nagelhout et al., 2011a). Overall, prevalence rates were shown to be higher among lower and middle educated groups compared to their counterparts. In 2010, $22 \%$ of those educated to a 
higher level smoked, whereas 30\% middle-level and 31\% lower-level educated people smoked. Additionally, SES differences in smoking were higher among women (30\% in HSES vs. $20 \%$ in LSES) compared to men (33\% in HSES vs. $24 \%$ in LSES) and most observable among people aged 25-44 (Nagelhout et al., 2011a). The large differences in smoking behaviour in the Netherlands make smoking an essential contributor to SES inequalities in health and give reason to develop programmes aimed at decreasing the number of smokers, especially in smokers educated to middle and lower levels.

Across the years, a number of different population-level tobacco control interventions such as bans on advertising, media campaigns and various health education programmes have been developed and implemented in an attempt to decrease smoking prevalence and to reduce SES differences in smoking behaviour (Kunst et al., 2004, Lemmens et al., 2008, Levy et al., 2004). Among population-level tobacco control interventions, cigarette tax increases have been considered as one of the most effective tobacco control interventions for reducing tobacco use (Levy et al., 2007, World Health Organization, 2010). Although tobacco tax increases have appeared to be successful in reducing tobacco consumption among LSES groups in the Netherlands and several other countries (Lee, 2008, Townsend et al., 1994), they may at the same time stimulate illegal tax evasion strategies, such as cross-border cigarette purchasing (Nagelhout et al., 2013). Evidence from national and international studies has indicated that further rises in tobacco prices may stimulate the smuggling of cigarettes, which could be seen by LSES groups as a way to deal with high prices (Nagelhout et al., 2013, Shelley et al., 2007, Wiltshire et al., 2001). Another policy concerns smoke-free legislation. Several laws that ban smoking in indoor workplaces and public places, such as restaurants and bars, have been implemented in the Netherlands. Although it is seen as an important policy under the WHO Framework Convention on Tobacco Control (FCTC) and was ratified by several countries, Dutch smoke-free legislation was shown to be less successful in decreasing tobacco consumption compared to other countries and was not particularly effective among LSES groups (Nagelhout et al., 2011b, World Health Organization, 2003). Another example of policies pertaining to health is that of placing warning messages on tobacco products, which may be effective in increasing knowledge and risk perception of smoking [28], although some authorities have expressed concerns about their effectiveness. The efficacy of this strategy may also be dependent on the degree of individuals' self-efficacy, which is often lower in LSES smokers (Peters et al., 2012, Siahpush et al., 2006). Since smoking behaviour is often determined by the social context, another way to reduce smoking is by the use of communitybased interventions. Yet, community-based interventions, aimed at reducing smoking in LSES communities, were not proven to be effective in producing higher cessation rates among the more disadvantaged groups in the Netherlands and several other countries (Bouwens et al. 2007, Fisher et al., 1998, Ronda et al., 2004, Secker-Walker et al., 2008, Stevens et al. 2002). 
The above mentioned findings show that most population-level interventions do not necessarily decrease consumption in LSES groups (Giskes et al., 2005, Schaap et al., 2008, Tariq et al., 2009). Ineffectiveness of these strategies in LSES smokers might be the result of a number of factors such as low exposure and attention to the intervention, low motivational impact, and the individual's inability to make behavioural changes in response to these strategies (Niederdeppe et al., 2008a, Niederdeppe et al., 2008b). Additionally, the higher proportion of smokers in LSES smokers' social networks and their lower social support may further reduce the chance of successful cessation (Chandola et al., 2004, Lindstrom et al., 2000). Possibly, socioeconomically deprived smokers may benefit from more intensive forms of smoking cessation support, such as programmes at a more individual level that help the person to quit smoking and to remain abstinent (Warner, 2008).

Different individual-level cessation interventions, such as individual and group counselling, nicotine replacement therapy, telephone counselling, smoking cessation counsellors and different Internet-based interventions have already been shown to be effective in supporting smokers when quitting smoking (Berndt et al., 2013, Civljak et al., 2013, Elfeddali et al., 2012a, Lancaster and Stead, 2005, Lemmens et al., 2008, Smit et al., 2012). Despite the existence of effective individual-level cessation interventions, their impact on LSES groups is likewise often suboptimal (Hiscock et al., 2012, Nevid et al., 1996). Up to the present, weak evidence has been found regarding the effectiveness of individual behavioural therapies among LSES groups (Bauld et al., 2010, Murray et al., 2009). Furthermore, Internet-based support has been shown to be especially effective for HSES smokers who are often more likely to engage in, adhere to and successfully quit as a result of these programs (McClure et al., 2006, Schaap et al., 2008, Seidman et al., 2010, Strecher et al., 2008a). In the Netherlands and other countries, however brief interventions were similarly unsuccessful in reducing smoking inequalities (Giskes et al., 2007). Given the sparse evidence regarding interventions that have succeeded in reducing high smoking prevalence rates among the LSES groups (Giskes et al., 2007, Hiscock et al., 2012, Nagelhout et al., 2012), there is a need for more smoking cessation programmes that help LSES smokers to quit smoking. This has stimulated the idea of developing a more personalised intervention aimed at increasing smoking cessation rates among LSES smokers and reducing SES inequalities in smoking in the Netherlands.

\section{Explaining socioeconomic inequalities in smoking}

In order to understand why people smoke, especially those of LSES, it is important to examine the underlying mechanisms and factors that may lead to an individual's decision to continue smoking, or to stop. Research on smoking cessation has been largely based on different theoretical models that emphasise the importance of a social cognitive approach. These models focus on the behaviour decision-making process and the underlying motives regarding 
smoking and smoking cessation (Ajzen, 1985, Bandura, 1986, De Vries et al., 2008, Prochaska and DiClemente, 1983). According to these health behaviour theories, the decision process is often determined by the individual's intention to perform a behaviour (Prochaska et al., 1997, Prochaska and DiClemente, 1983), which can be in turn influenced by several cognitive factors, such as knowledge, attitudes, social norms, perceived behavioural control and other personal resources (Fishbein and Ajzen, 1975, Fishbein and Ajzen, 2010).

During the last decades, various studies have revealed that LSES smokers often smoke more cigarettes per day and are more addicted, compared to HSES smokers (Fidler et al., 2008, Siahpush et al., 2006). Moreover, LSES smokers often have a lower intention/motivation to quit smoking (Siahpush et al., 2006) and are less successful in their quit attempts (Hiscock et al., 2011, Kotz and West, 2009). Similar findings, with regard to these SES differences have been identified in the Dutch setting (Nagelhout et al., 2011a, Nagelhout et al., 2012, Van Lenthe et al., 2004). Research studies have already examined the role of motivational factors in explaining socioeconomic differences with regard to smoking. Motivation to stop smoking has been found to be an important factor underlying smoking cessation. There is some evidence that LSES groups are often less likely to intend and have the desire to quit (Smit et al., 2011). This lower intention/motivation can be explained in terms of differences in attitudes, social influence beliefs and self-efficacy. A range of studies has indicated that LSES smokers are less convinced of the advantages of quitting (Dotinga et al., 2005, Lim et al., 2009, Manfredi et al., 2007, Rise et al., 2008); providing information about the benefits of quitting may therefore increase LSES people's motivation to change (Michie et al., 2009).

Smoking cessation is also often impeded by factors in the social environment of LSES groups; for example less favourable norms towards smoking cessation and more smoking in their environment (Chandola et al., 2004, Ellaway and Macintyre, 2009). Several studies have shown that socioeconomically deprived smokers experience more difficulties with quitting; they are less likely to quit when they perceive no support from their social environment (Chandola et al., 2004, Ellaway and Macintyre, 2009, Patterson et al., 2004). Additionally, LSES smokers are often more exposed to higher levels of stress and possess less effective strategies to cope with these stressors compared to their counter group (Gallo and Matthews, 2003). Identification of such barriers and an emphasis on social support may therefore be helpful to include in smoking cessation interventions targeting LSES smokers (Michie et al., 2009).

A successful quit attempt may be also determined by the individual's perceived belief in his or her ability to quit smoking. Studies observing this pattern found that LSES smokers repeatedly showed a lower sense of self-efficacy, which is often associated with relapse (Siahpush et al., 2006). Another reason for a lower rate of smoking cessation in LSES groups may be the lack of successful action planning to prepare and cope with difficult situations that may trigger relapse. In general, action planning has shown to be effective for smoking cessation, especially when action plans are also enacted upon (De Vries et al., 2013). The 
formulation of realistic goals, as well as the identification of several barriers, have already proved to be helpful for people of LSES by helping them to translate their goals into actions and should therefore be included in smoking cessation interventions (Michie et al., 2009).

To summarise, it is possible that LSES smokers may not receive adequate support, adapted to their individual situation. Additionally, they may not be sufficiently convinced of the advantages of quitting, and may be given low levels of support for quitting by their social environment as well as possessing low self-efficacy and making inadequate action plans. In order to offer effective and feasible support to LSES smokers, improved smoking cessation interventions based upon the above-mentioned factors are therefore necessary to improve smoking cessation interventions, targeting LSES smokers.

\section{Web-based computer-tailored smoking cessation interventions for LSES groups: a challenge}

Because of the high level of reach and availability of the Internet, more and more smoking cessation interventions are delivered through the Web (Civljak et al., 2013). Web-based computer-tailored smoking cessation interventions have already been shown to be effective (Civljak et al., 2010, Civljak et al., 2013, Etter, 2005, Shahab and McEwen, 2009, Strecher et al., 2008b) and may provide an opportunity for quitting smoking in different SES groups.

In computer-tailored smoking cessation interventions, smokers are provided with personalised and unique information on their smoking behaviour. Computer tailoring can be defined as a communication strategy in which personal data related to a specific health outcome is assembled, and personalised information is then given based on this specific data (Rimer and Kreuter, 2006). This personal information is gathered by means of the individual's answers to a questionnaire about his or her smoking behaviour, beliefs, social support, selfefficacy, motivation to change and other circumstances (De Vries and Brug, 1999). Usually, computer-tailored interventions entail a screening instrument which assembles the personal information by a questionnaire related to the person's behaviour. Next, the personal data collected during the questionnaire is matched with feedback messages from a file, consisting of all possible feedback answers. A computer software program combines the relevant feedback messages with the personal answers given in the questionnaire to create a personal tailored feedback advice. In this way, the person receives only information relevant to his or her personal situation (Dijkstra and De Vries, 1999). Over the years, tailored information has been shown to be more likely to be read, remembered and regarded as personally important, compared to non-tailored information (Brug et al., 1999, De Vries and Brug, 1999, Kreuter et al., 1999a, Ruiter et al., 2006). Next to this, tailored messages also appear to stimulate a deeper processing of the health information (Dijkstra, 2005, Kreuter and Wray, 2003, Petty et al., 1981). While past research has shown that a single section of tailored smoking advice was 
effective in increasing abstinence rates, the effects of tailoring might be further increased by providing multiple tailored feedback moments (Borland et al., 2004, Dijkstra et al., 1998a, Smit et al., 2012). Within multiple computer-tailored interventions, feedback can be provided at multiple moments in time, which is assumed to be essential in order to realise sustainable changes in health behaviour (Lenert et al., 2003, Wantland et al., 2004). Research has also shown, however, that intensive interventions might cause an overload of information which may have a negative impact on respondents' participation (Elfeddali et al., 2012a).

Although web-based computer-tailored smoking cessation interventions seem to be a promising strategy to help smokers to quit smoking, they offer a number of problems and challenges. Firstly, despite expectations, the reach of these interventions is often suboptimal (Brouwer et al., 2010). Although, it is expected that a large number of people can be reached, the actual reach of these web-based interventions is often inadequate (Brouwer et al., 2010, Evers et al., 2005, Eysenbach, 2005). Secondly, attrition from web-based intervention sessions is a common problem (Eysenbach, 2005). In particular, LSES groups often leave the program before completing all the intervention elements and show a lower adherence towards these programs compared to HSES groups (Strecher et al., 2008b, Warren-Findlow et al., 2003). Furthermore, LSES smokers, who are often unmotivated to quit smoking, are often not included in these programs since most of the interventions have been developed for smokers motivated to quit (Civljak et al., 2013, Lancaster and Stead, 2005).

An essential step in improving the reach, exposure and adherence to web-based computer-tailored smoking cessation interventions among LSES and HSES smokers is to identify important determinants of these factors, which will be the focus of this dissertation. In the following section, the different problems will be described in detail and then used to explain the rationale for the most important decisions made in the development of our smoking cessation intervention for LSES and HSES smokers.

\section{How to improve the reach, adherence and effectiveness of computer-tailored smoking cessation interventions in LSES and HSES groups?}

Over the past years, the many advantages of the Internet have made it a promising channel to deliver health-related interventions. Since more and more Dutch households use the Internet, it is a powerful way to reach a large part of the population, with relatively low costs (Strecher, 2007). Recent statistics in the Netherlands have shown that the Internet is highly accessible to LSES smokers (more than 90\% have Internet access), and medium and HSES smokers (more than 98\% have Internet access) (CBS, 2011a). Another advantage of web-based interventions is that they are easily accessible, enabling people with Internet access to use the intervention anonymously anytime and anywhere (Bennett and Glasgow, 2009). Since the Internet is a 
useful medium for the delivery of these interventions, web-based computer-tailored smoking cessation interventions make it possible to combine an individual approach with a large reach.

Despite the usefulness of the Internet, previous research has indicated that only a small part of the population is actually reached by these interventions. As already mentioned above, people of HSES with a healthier lifestyle pattern tend to make more use of these interventions (Brouwer et al., 2011, Brouwer et al., 2010, Crutzen, 2009a). However, since especially LSES smokers in the Netherlands show the highest addiction levels and experience most problems in quitting (Nagelhout et al., 2012, Peters et al. 2005), more attention should be paid to how best to target this particular segment of the smoking population. Past research has indicated that large Internet campaigns have the potential to reach a large group with relatively low costs, but its actual reach among LSES and HSES groups is not clear (Swartz, et al., 2006). Recruitment via general practitioner (GP) practices was successful in reaching lower educated smokers in particular, although the net effect upon those trying to stop smoking still favoured the mass media approach (Smit, et al., 2011). To enhance the public health impact of webbased computer-tailored smoking cessation interventions, it is important to identify the strategies that are effective in recruiting different SES groups, especially LSES groups. The research described in this dissertation therefore tested four different recruitment channels to investigate the dissemination and reach of a web-based computer-tailored smoking cessation intervention among LSES and HSES smokers.

In order to improve the overall impact of computer-tailored smoking cessation interventions among different SES groups, it is not only important to investigate the reach of an intervention, but also to assess its use and exposure towards the intervention and provide recommendations for optimalisation (Leslie et al., 2005). Although web-based delivered smoking cessation interventions have shown to be effective, results from past studies have indicated a relatively low adherence towards these programs (Strecher et al., 2008a, WarrenFindlow et al., 2003). In addition, it is often difficult to maintain respondent loyalty (future adherence) towards an intervention (Crutzen et al. 2011); loyalty may be necessary since Internet delivered behaviour change interventions often require repeated visits (Verheijden et al., 2007, Wantland et al., 2004). Internet interventions implemented in a real life setting often have to deal with low exposure rates (Evers et al., 2005), since respondents often choose to access only a limited part of the program (Eysenbach, 2005, Martinez, 2003, Tyler-Smith, 2006). Rates of engagement and completion of these smoking cessation interventions are even lower in high-risk groups such as LSES smokers (Moser et al., 2000, Strecher et al., 2008a, Warren-Findlow et al., 2003). To achieve sustainable changes in health behaviours, it is necessary that respondents follow the most important parts of the intervention, pay attention to the different intervention components, and internalize the health-related messages (McGuire, 1985). Consequently, it is essential to investigate how LSES and HSES smokers actually use these interventions, whether they intensely process the health-related 
information and, whether they are likely to revisit the Internet intervention (Crutzen et al., 2011). Therefore, this dissertation aims to investigate factors stimulating processing mechanisms of the health message, and future adherence in order to optimise computertailored smoking cessation interventions among different SES groups.

Up to now, most computer-tailored smoking cessation interventions have been developed for smokers highly motivated to quit (Civljak et al., 2013, Echer and Barreto, 2008); LSES smokers often show low motivation, however, and may benefit from interventions which give them the possibility and time to reflect on their smoking behaviour and intention to quit at the time when they are ready to prepare for their quit attempt. As a result, web-based computer-tailored smoking cessation interventions should be adapted to the needs of groups with different levels of education and should take into account the user's motivation to quit.

At the present, web-based computer-tailored smoking cessation interventions are mainly based on simple text-based messages. Since Internet users often scan a text for relevant information, such interventions may not be sufficiently attractive for smokers, especially LSES smokers (Liu, 2005, Vandelanotte and Mummery, 2011). Since more and more websites increasingly make use of interactive elements such as graphics or videos to increase their attractiveness (Purcell, 2010), it is considered fruitful to apply such elements in a computertailored intervention for several reasons. Firstly, past research has indicated that video-based messages might require less mental effort and might help the person to concentrate on the most important elements of the message (Salomon, 1984, Salomon and Gardner, 1986). As suggested by previous research, video messages may activate both visual and verbal channels and may therefore lead to better processing of the messages (Mayer and Massa, 2003). Secondly, different studies have shown that the processing of information in LSES groups might be more influenced by visual information (Campbell et al., 1999, Danaher et al., 2005, Houts et al., 2006). Findings using the Elaboration Likelihood Model indicated that LSES groups often do not process the information deeply (Cacioppo et al., 1996). As video messages use images that do not require the translation of abstract concepts into concrete actions, LSES groups may profit from video information (Krouse, 2001). Video messages may help to focus the person more on the basic element of the message (Mayer and Massa, 2003). As the video content might improve the appeal of health interventions, the integration of video messages might be a promising strategy to attract and engage people, especially those of with a LSES (Calvert et al., 2005, Ma et al., 2005). To date, little is known about the specific effect of tailored video messages on smoking cessation among different SES groups. To clarify the full potential of these interventions, further research is required to investigate the degree to which these interventions are effective among smokers with different SES and their short- and long-term effects in a real-life setting. Therefore, this dissertation aims at testing video- and text-based tailored messages for smoking cessation in LSES and HSES smokers. 


\section{This dissertation}

Development of two web-based multiple computer-tailored smoking cessation interventions As mentioned earlier, there are indications that LSES smokers may profit from video information and from an intervention that is also suitable for those who are less motivated to quit. Hence, two multiple web-based computer-tailored smoking cessation interventions were developed, incorporating two different delivery approaches (video- vs. text-based computertailored messages) to determine which intervention is most effective in promoting smoking cessation among LSES and HSES smokers. One of the interventions provided respondents with video-based messages whereas the other intervention provided respondents with text-based messages. The content of the two interventions was exactly the same to test the added effect of one of the two delivery strategies. In the video-based intervention, the tailored advice was translated into videos. The video-based messages were delivered by five different adults in a TV 'news program' format. Furthermore, the intervention entailed two different motivational routings tailored to the smoker's motivation to quit. A more detailed description of the two interventions can be found in Chapter 2 of this dissertation (Stanczyk et al. 2011). The following section will describe the theoretical framework as well as details of the content of the two computer-tailored smoking cessation interventions.

\section{The theoretical framework and content of the smoking cessation interventions}

The two multiple web-based computer-tailored smoking cessation interventions were based mainly on the I-Change model (ICM) (De Vries et al., 2003) (Figure 1). A predecessor of the ICM was the Attitude-Social-Influence-Efficacy (ASE) Model (De Vries and Mudde, 1998) which has been applied in several Dutch smoking cessation studies (Ausems et al., 2002, Bolman and De Vries, 1998). The ICM includes different theoretical concepts from different cognitive models, such as the Transtheoretical Model (TTM) (Prochaska et al., 1997), the Theory of Planned Behaviour (TPB) (Ajzen, 1985), the Social Cognitive Theory (SCT) (Bandura, 1986), and the Health Belief Model (HBM) (Janz et al., 2002). In the ICM, intention is seen as the most important proximal predictor of a particular health behaviour and is divided into three phases: precontemplation, contemplation, and preparation. 


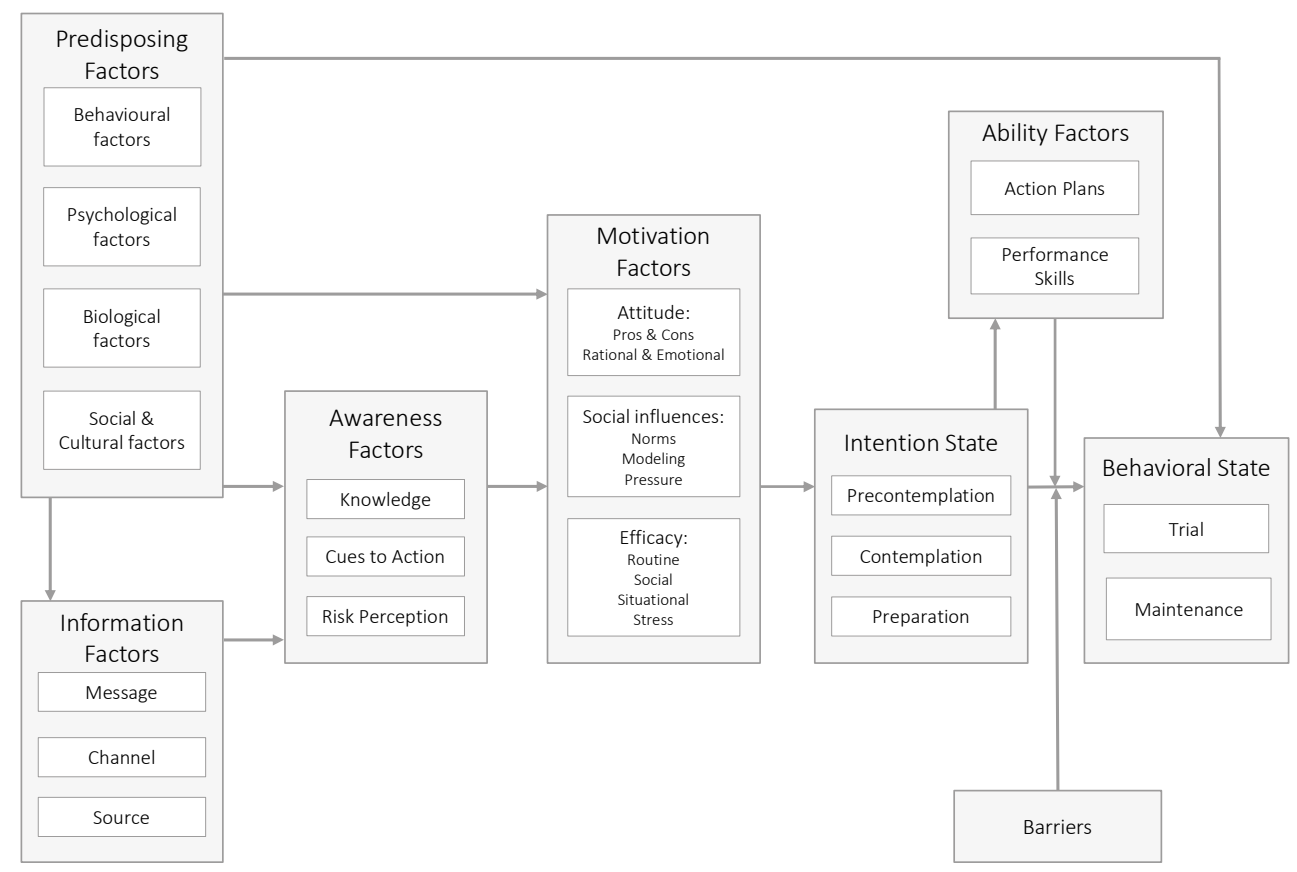

Figure 1 The I-Change Model (ICM)

The ICM further hypothesises that intention for behaviour change is predicted by three different motivational concepts: attitude, social influence, and self-efficacy. The ICM proposes that attitude consists of perceived advantages (pros) and disadvantages (cons) of a health behaviour. Social influence includes three different concepts: social norms, social modeling, and social pressure. Social norms refer to the perceived opinions of others within the person's environment, such as friends, family and the partner. Social modelling is regarded as the perceived behaviour of others around the person, whereas social pressure refers to the perceived pressure from others to execute a certain behaviour. Self-efficacy refers to a person's level of confidence that he or she is able to perform a specific behaviour. Research on smoking behaviour has shown that these motivational factors are important predictors of the intention to quit smoking, and are therefore included in the current intervention (De Vries and Mudde, 1998, Hoving et al., 2006a, Rise et al., 2008).

According to the ICM, the motivational concepts of attitude, social influence and selfefficacy are influenced by different pre-motivational factors. Awareness factors influence motivation and incorporate factors such as knowledge, cues to action, and risk perception. Furthermore, motivational processes are also determined by predisposing factors referring to behavioural psychological, biological and social-cultural factors. Additionally, these motivational factors can be also influenced via health communication by which its effectiveness is determined by various message, channel and source determinants. In line with 
other self-management theories that stress the importance of post-motivational processes (Cohen et al., 2008, Sheeran et al., 2005, Sniehotta et al., 2005), the ICM suggests that there often exists a discrepancy between the intention to change a special behaviour and the actual performance of the behaviour; this is known as the intention-behaviour gap (Armitage and Conner, 2001, Sniehotta et al., 2005). According to the ICM, different post-motivational factors are important in order to bridge the gap between intention and the health behaviour, as follows. Firstly, specific perceived barriers to execute the behaviour may increase the intention-behaviour gap. However, different ability factors, such as the person's individual skills and action plans can enable him or her to cope with these barriers; the result may be a decrease in the gap between intention and behaviour. According to the ICM, action plans are subdivided into preparatory plans and coping plans. Preparatory plans refer to planning actions, needed to perform a new behaviour for the first time, whereas coping plans refer to planning actions, in order to cope with barriers and relapse and to maintain the realised changes. Past research has already found evidence that preparatory and coping plans are effective in increasing quit attempts and actual smoking cessation (De Vries et al., 2013, Elfeddali et al., 2012a). An important element of action planning is action enactment, implying that action plans are effectively executed (De Vries et al., 2013).

The two web-based multiple computer-tailored smoking cessation interventions are partly based on two previously effective computer-tailored smoking cessation interventions (Smit et al., 2012, Te Poel et al., 2009). An important extension implied the development of a routing for smokers motivated to quit within one month, and a routing for smokers not motivated to quit within the next month, allowing the latter group more time to become motivated and to prepare for their quit attempt. Since LSES smokers are often less motivated to quit and most cessation interventions are often developed for highly motivated smokers, we decided to develop two different motivational routings in our smoking cessation intervention to give less motivated smokers the possibility and time to reflect on their intention to quit and to prepare successfully for quitting. After filling in the baseline questionnaire, respondents received their first tailored feedback (session 1), including feedback about their smoking behaviour, attitude, perceived self-efficacy, and how to plan a quit date. Depending on their readiness to plan a quit date within the following month, respondents received tailored feedback during several sessions and were directed either into routing 1 or 2 (see Figure 2). After each feedback session, respondents were provided by e-mail with an overview of their tailored feedback. There follows a brief description of the two different routings.

\section{Routing 1}

Respondents who were ready to quit smoking within the following month were directed into routing 1 , which contained six feedback moments. The aim of this routing was to help smokers to enable the translation into action of their intention to quit smoking within one month by 
providing personalised feedback messages. After the first session, respondents were asked to set a quit date within the next month. One week before their quit date, respondents were invited by e-mail to follow the second session. They received feedback on the extent to which they had already made concrete plans for their quit attempt (preparatory planning), since research has shown that preparation increases the chances of quitting (Te Poel et al., 2009). Additionally, they received feedback on their perceived self-efficacy to quit, including feedback on how to deal with barriers and risk situations (coping planning). As recent studies have found that ex-smokers often relapse shortly after their quit attempt, respondents were provided with different relapse prevention strategies (Elfeddali et al., 2012b, Hughes et al., 2004). During the third session (three days after the quit date), respondents received tailored feedback on their perceived self-efficacy and feedback on how to cope with risk situations. During the fourth session (two weeks after the quit date), respondents received personalised feedback on their self-efficacy, including feedback on how to deal with difficult situations and their attitude towards smoking and quitting. Four and eight weeks after their quit date, respondents were again invited to follow sessions five and six; during these two sessions a similar strategy was used as in session four.

\section{Routing 2}

Respondents who were not ready to quit smoking within the following month were directed into routing 2 , which contained three feedback moments. The aim of routing 2 was to increase the smoker's motivation by increasing perceptions of the pros of quitting and how to obtain support for quitting. During the first session, (directly after the baseline questionnaire) smokers were encouraged to use the next month to reflect on their intention to quit smoking. During the second session (one month after the baseline questionnaire), respondents received tailored feedback on their smoking behaviour, different cognitive variables, such as their attitude (the pros and cons of smoking and quitting), their perceived social support, and their readiness to quit smoking. If they were ready to quit within one month, they were directed to routing 1 . 


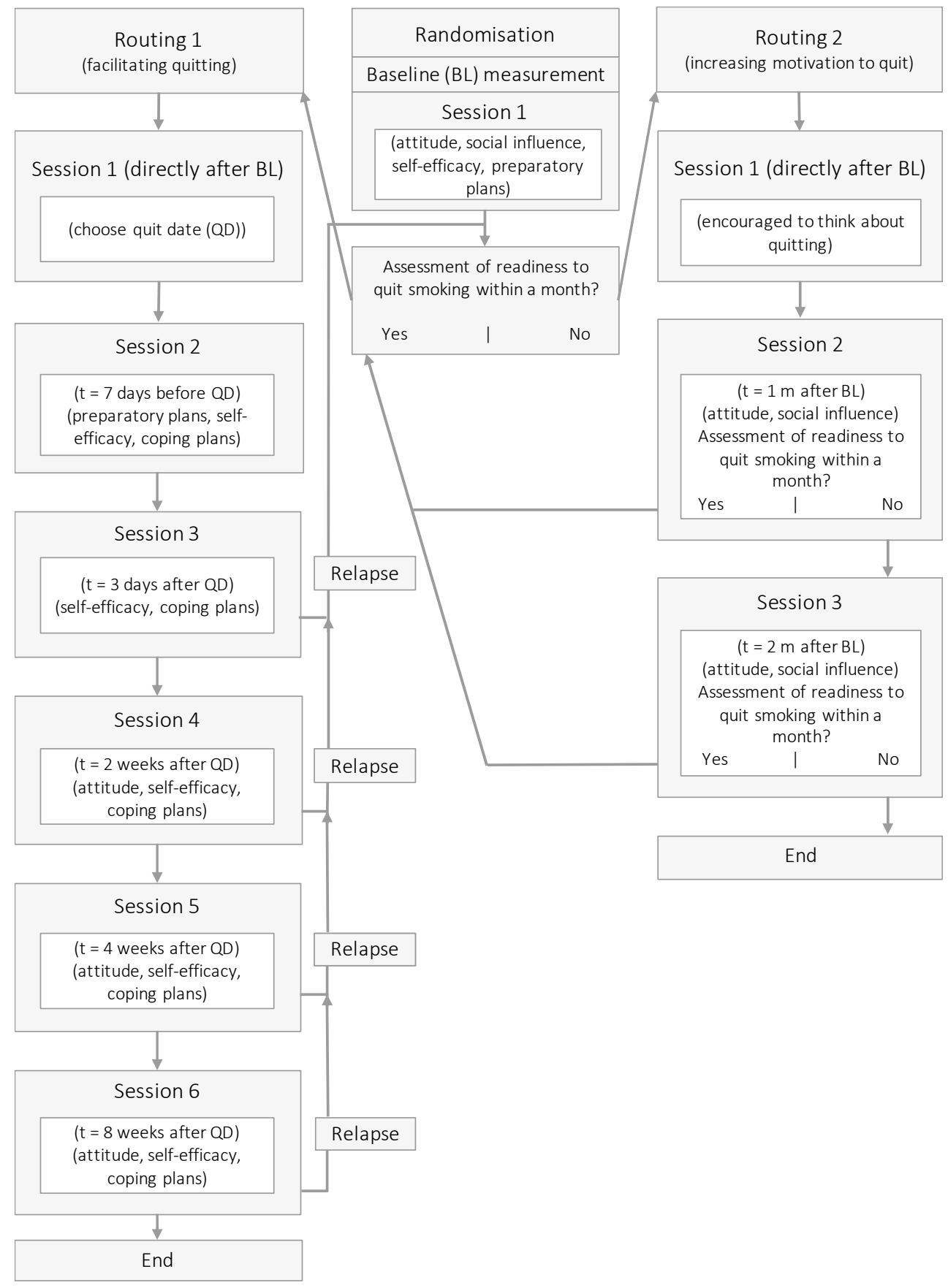

Figure 2 Intervention design for the video and text tailored intervention, following 2 routings 
Respondents not ready to quit after one month again received an invitation to follow the next session (session 3), by means of a similar strategy used in session 2. If, at the end of session 3 , respondents were not prepared to quit they received an e-mail indicating that we would send no further invitations and that the intervention would end at this stage. In the following section, a brief overview is provided of the different studies conducted to investigate the reach, adherence, processing and (cost) effectiveness of the web-based computer-tailored smoking cessation intervention.

\section{Rationale and aim of the dissertation}

\section{Reach of the computer-tailored intervention}

To recruit different SES smokers for participation in the computer-tailored smoking cessation intervention, four different recruitment strategies were used (Dutch GP practices, newspaper advertisement, Internet advertisement and other strategies, such as e-mailing to companies and referrals). This procedure enabled us to investigate whether different recruitment strategies resulted in different samples of smokers with regard to sample size as well as demographic and smoking-related characteristics. Moreover, we were able to investigate whether possible differences existed between the four differently recruited samples regarding retention rates. As mentioned before, this might be especially important, since web-based interventions often have to deal with high attrition rates (Blankers et al., 2010, Eysenbach, 2005, Shahab et al., 2009, Webb, 2009). We were also able to assess whether the choice for a particular recruitment strategy resulted in differences among the four different samples regarding quit attempts and smoking abstinence. The first study of this dissertation (Chapter 3) therefore aimed to answer the following research questions:

1) Do the different four recruitment strategies for participation in the computertailored smoking cessation intervention result in different sample sizes, different characteristics and different socioeconomic groups of smokers?

2) Do significant differences exist between the four recruited samples regarding retention rates after six month follow-up measurements?

3) Do significant differences exist between the four recruited samples regarding smoking behaviour (quit attempts and smoking abstinence) after six month followup measurements? 


\section{Future adherence and processing towards the computer-tailored intervention}

As mentioned in the previous section, evidence from different studies indicates that the actual use and exposure rates of health-related interventions are often low (De Nooier et al., 2005). Exposure rates might be even lower among LSES groups (Moser et al., 2000, Strecher et al., 2008a). Respondents often spend a limited amount of time using the intervention, which makes optimal exposure not feasible. Health communication research showed that different underlying message-processing mechanisms play an important role in enhancing the healthrelated messages, namely: attention, comprehension, processing, self-reference, and appreciation (Hawkins et al. 2008). The effects of these tailoring processing mechanisms might be further strengthened by the use of an appropriate delivery strategy (video- vs. text-based messages). Additionally, future adherence/loyalty might be also increased by a suitable delivery strategy (video- vs. text-based messages). The second study of this dissertation (Chapter 4) aimed to assess whether differences exist in message-processing mechanisms and future adherence, according to delivery strategy and educational level. By this study it was aimed to answer the following research questions:

1) Do the effects of message-processing mechanisms according to a different delivery strategy (video-vs. text-based messages) differ among LSES and HSES smokers?

2) Do the effects of future adherence according to a different delivery strategy (videovs. text-based messages) differ among LSES and HSES smokers?

\section{(Cost) effectiveness evaluation of the computer-tailored intervention}

Effective smoking cessation interventions can influence the population's health by reducing death and smoking-related illnesses (World Health Organization, 2011). The intervention's effect is ideally measured by randomly assigning respondents to an intervention group or a control group (respondents who will not receive the intervention). As a third and fourth study of this dissertation (described in Chapters 5 and 6) a randomized controlled trial (RCT) was conducted within the Dutch smoking population to test the effectiveness of the two versions of the multiple computer-tailored interventions compared to a control condition at six and 12 months follow-up. Respondents in the two experimental conditions received either several text-based or video-based messages. Respondents in the control condition received a generic text advice about quitting smoking at the end of their baseline questionnaire. During the whole study period, respondents also were able to use other smoking cessation aids. As mentioned earlier, we expected video-based messages to be more successful in LSES smokers, since the processing of information in LSES groups might be more influenced by visual information (Danaher et al., 2005). Conversely, we expected that text-based messages may be more suitable for HSES smokers who might profit more from in-depth processing of the message. To explore possible differential intervention effects for different SES groups, we took different SES 
measures into account (educational level and occupational status) when examining long-term abstinence. Additionally, in both the video- and text-based intervention, smokers were provided with the opportunity to follow different motivational routings, adapted to their readiness to quit smoking. Consequently, there is a need to assess the success of the two interventions in respondents with a lower motivation to quit smoking. The effectiveness of the intervention was measured by two different measures of smoking abstinence: prolonged abstinence and seven-day point prevalence abstinence.

Finally, in times of rising health-care costs and limited funds in the Netherlands, economic evaluations of computer-tailored smoking cessation interventions are needed in order to choose the most cost-effective interventions (Evers et al., 2010). Different economic evaluations of smoking cessation interventions have already shown that these interventions are cost-effective (Feenstra et al., 2005, Mudde et al., 1996, Song et al., 2002, Woolacott et al., 2002). A recent study in the Netherlands has reported that a web-based computer-tailored smoking cessation intervention in a GP setting seemed to be more cost-effective regarding smoking abstinence, compared to usual smoking cessation care (Smit et al., 2013). The fifth study (Chapter 7) of this dissertation aimed to investigate the cost-effectiveness of the two web-based computer-tailored smoking cessation interventions. With regard to the effectiveness and cost-effectiveness of the video-based and text-based intervention on smoking abstinence, the dissertation aimed to answer the following research questions:

1) Do the two multiple computer-tailored smoking cessation interventions (video vs. text condition) have significant effects after six and 12 months on smoking abstinence when compared to a control condition among a sample of Dutch smokers?

2) Are there different effects of the intervention for respondents with different SES levels, and for respondents varying in their motivation to quit smoking?

3) Is the video- or the text-based computer-tailored smoking cessation intervention more cost-effective, when compared to each other and when compared to the control condition?

\section{Outline of the dissertation}

Chapter 2 provides a detailed description of the multiple computer-tailored smoking cessation intervention (see also Table 1). Chapter 3 describes the impact of recruitment method on the reach and retention of the intervention. Chapter 4 presents the results of a study investigating message-processing mechanisms and future adherence regarding the two web-based multiple computer-tailored smoking cessation interventions. Chapter 5 provides the results of the sixmonth effectiveness study of the intervention among a sample of Dutch smokers, motivated to quit smoking within six months. Chapter 6 describes the 12-month effectiveness study of the intervention among a sample of Dutch smokers. Chapter 7 presents the results from the 
economic evaluation that was executed to assess the cost-effectiveness and cost-utility of the web-based computer-tailored smoking cessation intervention. In the last chapter of this dissertation, Chapter 8 , the results from all the different chapters are combined and discussed, and suggestions are made for future smoking cessation studies. 


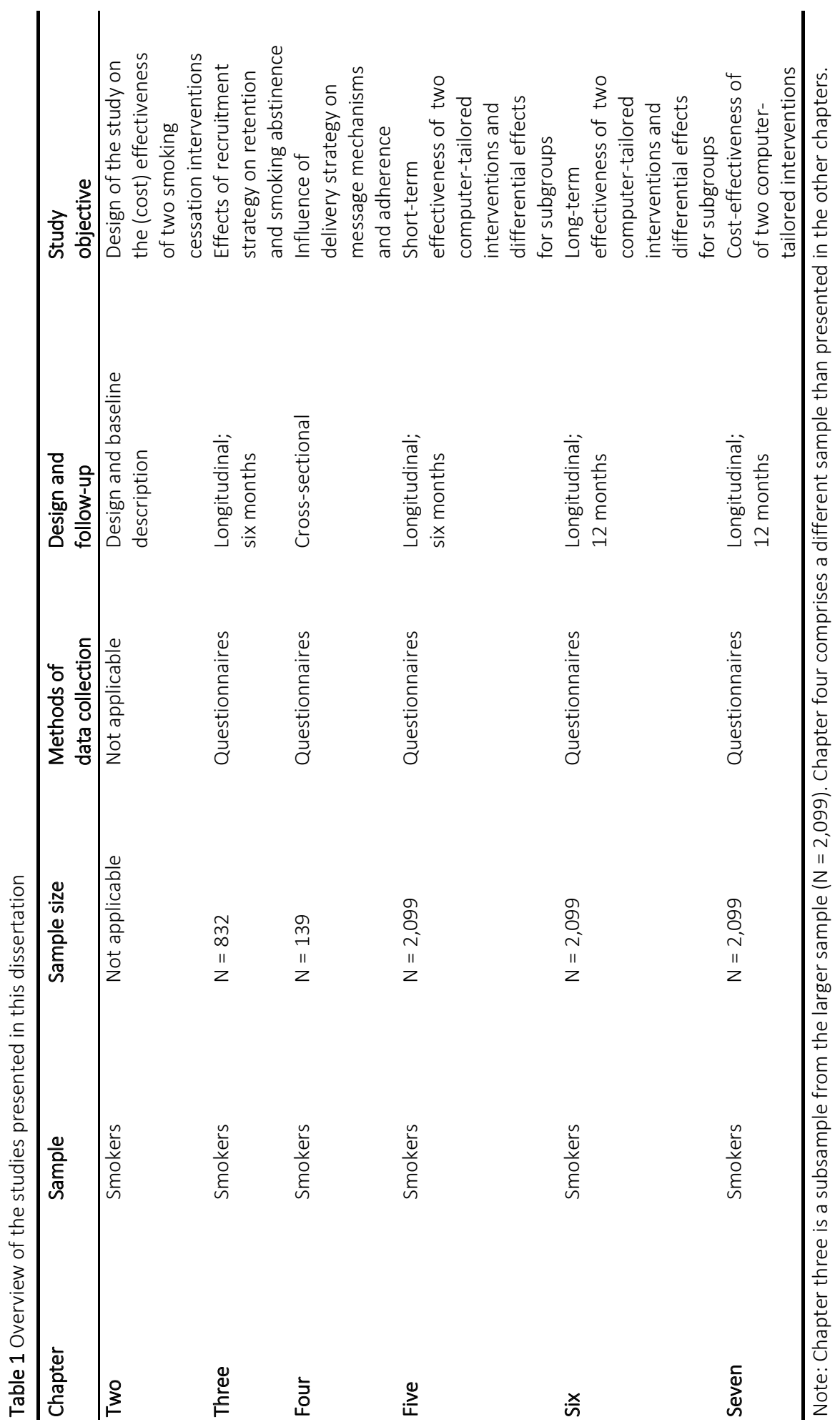




\section{CHAPTER 2}

Study protocol of a Dutch smoking cessation e-health program

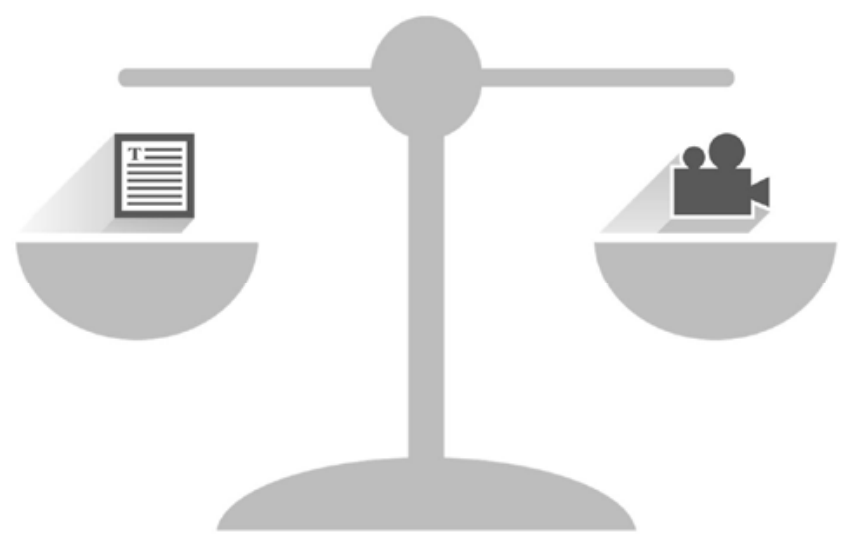

Published as:

Stanczyk, N. E., Bolman, C., Muris, J. W. M. \& de Vries, H. (2011). Study protocol of a Dutch smoking cessation e-health program. BMC Public Health, 11, 847. 


\begin{abstract}
Background: The study aims to test the differential effects of a web-based text and a webbased video-driven computer-tailored approach for lower socioeconomic status (LSES) and higher socioeconomic status (HSES) smokers which incorporate multiple computer-tailored feedback moments. The two programs differ only in the mode of delivery (video- vs. text-based messages). The paper aims to describe the development and design of the two computertailored programs.
\end{abstract}

Methods/Design: Respondents who smoked at the time of the study inclusion, who were motivated to quit within the following six months and who were aged 18 or older were included in the program. The study is a randomized control trial with a two (video/text) * two (LSES/HSES) design. Respondents were assigned either to one of the intervention groups (text vs. video tailored feedback) or to the control group (non-tailored generic advice). In all three conditions respondents were asked to fill in the baseline questionnaire based on the I-Change model. The questionnaire assessed socio-demographics, attitude towards smoking, knowledge, self-efficacy, social influence, depression, level of addiction, action planning, goal actions, intention to quit smoking, seven-day point prevalence and continued abstinence. Follow-up measurements were conducted at six- and 12-months after baseline.

Discussion: The present paper describes the development of the two computer-tailored smoking cessation programs, their components and the design of the study. The study results reveal different working mechanisms of multiple tailored smoking cessation interventions and will help us to gain more insight into effective strategies to target different subgroups, especially smokers with a LSES (Dutch Trial Register NTR3102). 


\section{Background}

Smoking tobacco is one of the most preventable causes of illness and premature death in the world (CDC, 2004). The development of effective smoking cessation programs is essential to prevent illnesses (Fiore et al., 2008).

One effective strategy for health promotion that has been developed during the last decades concerns computer-tailored interventions (De Vries and Brug, 1999, Lustria et al., 2009). Computer tailoring has developed since the 1990s as a new technique for health promotion, as it provides the individual with personalised information and feedback on health behaviour. Tailored health messages are based on knowledge of a person generated from his or her answers to a questionnaire on issues related to health behaviour (in the case of smoking, for example, the perceived advantages of smoking and perceived support to quit smoking). Research has demonstrated that tailored communication attracts and keeps the individual's attention (Kreuter et al., 1999a). Tailored information has furthermore been shown to be more likely to be read, remembered and considered personally relevant (Smeets et al., 2008). Computer-tailored programs can be either print delivered (delivered by post) or web-based. One advantage of web-based computer-tailored intervention is that the user can follow the program in privacy at any preferred time (Oenema et al., 2008). Furthermore, multimedia components can be incorporated and it has the potential to reach large audiences. Computer tailoring is therefore increasingly offered by the web.

In the Netherlands, a range of empirical studies has demonstrated the efficacy of webbased computer-tailored programs compared with non-tailored programs for several lifestyle behaviours (Smeets et al., 2008, Te Poel et al., 2009) including smoking cessation (Dijkstra and De Vries, 2000a, Dijkstra et al., 1998b, Dijkstra et al., 1999, Te Poel et al., 2009). The effectiveness of computer-tailored technology to smoking cessation has been reviewed and tested in various studies (Lancaster and Stead, 2005, Shahab and Mc Ewen, 2009).

Smoking prevalence in Dutch people with a low socioeconomic status (LSES) is higher than in people with a high socioeconomic status (HSES) (CBS, 2011a). People with a low SES also begin to smoke at an earlier age (Michie et al., 2009) and have more difficulties in quitting (Bell et al., 2006). Several studies suggest that health communication probably needs to be different for LSES populations and suggest an approach with less cognitive effort (Brouwner et al., 1996, Haug et al., 2010, Murphy et al., 2000) that focuses more on visual than on text information (Cacioppo et al., 1996).

Consequently, the potential problem with computer-tailored smoking cessation programs is that they rely heavily on text-based messages and might therefore be less attractive to LSES smokers. Cognitive psychologists emphasize learning from more than one modality source, since humans actively process information with separate systems for visual and verbal representations (Mayer and Massa, 2003). Several studies suggest that videos may 
be more effective in attracting attention and stimulating comprehension, especially in LSES smokers and smokers unmotivated to change (Nielsen and Sheppard, 1988). Video messages have been shown to require less mental effort and less translation of abstract concepts to create images (Salomon, 1984). Findings using the Elaboration Likelihood Model suggest that LSES groups often do not process information deeply (Cacioppo et al., 1996). Furthermore, research has identified that video presentation activates both visual and verbal channels, which together may lead to better learning outcomes (Mayer and Massa, 2003). Whereas textbased messages require translation of abstract ideas to concrete situations, video messages may help to focus the recipient more on the basic message and help their understanding of the core message and reasons for engaging in the desired behaviour. Lower educated and motivated groups may thus profit in various ways from the utilization of videos and illustrations in smoking cessation programs.

To test the effectiveness of video-based messages it is important to use an experimental design in which the information provided by the videos is comparable to the textbased messages and a control condition. The video information should therefore use the same basic messages as the text version (Tversky et al., 2002). Yet, as far as we know no studies have been executed which test whether the use of video-based messages alone (without any other animation effects, like cartoons, hyperlinks, etc.) has an added effect on smoking cessation especially when targeting less literate groups. Also little attention has been given yet to the possible efficacy of video-based computer-tailored smoking cessation interventions targeting LSES smokers.

Besides method strategy it is also important to consider the dosage of computer tailoring. Research has demonstrated that multiple smoking cessation computer tailoring results in greater cessation effects than single tailored feedback letters (Dijkstra et al., 1998b). Similar effects have also been found for other behaviours than smoking, e.g. physical activity and dietary behaviour (De Vries et al., 2008, Oenema et al., 2008). Multiple tailoring feedback moments are therefore considered to be a useful strategy to implement for web-based computer-tailored smoking cessation programs as well.

The study whose protocol is described here aims to include these different elements by developing and testing two new e-health programs, incorporating multiple computertailored feedback moments. The two e-health programs differed only in the mode of delivery that was used (video- vs. text-based messages). The main aim of this paper is to describe the development of the two computer-tailored smoking cessation programs, their components and the design of the efficacy study. 


\section{Methods}

The study protocol was submitted for approval to the Medical Research Ethics Committee (MREC) of Atrium Medical Centre Heerlen. The MREC reviewed the research protocol and judged that no further MREC approval was necessary for this study because patients were not obliged to a certain act. Furthermore, the questionnaires of the intervention were judged not to have a deep psychological impact. Human subjects' approval was obtained in line with the American Psychological Association (APA) informed consent ethical principles. At the beginning of the study, all eligible respondents were provided with information on the study and asked to sign informed consent forms.

\section{Target population and Inclusion criteria}

Only respondents who smoked at the time of the study inclusion, who were motivated to quit within the following six months and who were aged 18 or older were included in the study. Furthermore, respondents needed to have Internet access and had to be able to understand the Dutch language sufficiently.

\section{Study design}

Respondents were randomly assigned to one of the two experimental conditions or the control condition.

1) Text computer tailoring: respondents received computer-tailored text messages during several feedback sessions. The number of feedback sessions was dependent on the respondents' intention to quit smoking and their readiness to set a quit date within a month.

2) Video computer tailoring: respondents received computer-tailored video messages during several feedback sessions. The number of feedback sessions was dependent on the respondents' intention to quit smoking and their readiness to set a quit date within a month (the text messages mentioned in point one were replaced by videos).

3) Control group: respondents received one short piece of generic text advice. More detailed information is provided in the 'Intervention' section.

\section{Procedure}

Recruitment

Respondents were recruited by several recruitment strategies. First, smokers were recruited through general practitioner (GP) practices. We asked about $150 \mathrm{GP}$ practices to refer smoking patients to our intervention website. The GP practices received a letter which entailed a description of the project, tasks for the GP and the assistant and all necessary recruitment 
materials. GPs willing to participate in our study were asked to refer a minimum of 20 patients who met the inclusion criteria to the intervention website over a period of 12 months.

Second, respondents were recruited by a mass media approach, which consisted of calls in local newspapers and websites of national health funds (the Dutch Diabetes Foundation, the Dutch Cancer Society, the Dutch Foundation for a Smoke-free Future and the Dutch Asthma Foundation). The calls directly referred smokers who were interested in participating in the study to the intervention website where they could find more information about the project and registration.

Third, smokers were also recruited via several Dutch companies. We asked companies to bring the program to the attention of their employees by means of advertisements, announcements in staff magazines, internal websites and/or by any other channels they used to communicate with their employees. Similarly to the other recruitment strategies, calls referred employees to the intervention website.

The intervention website gave information about the content of the program and explained that three different versions of the program were to be tested. Respondents were informed that they would be randomly allocated to one of the three different program versions. Respondents were told that they could win $€ 100$ after completing all parts of the program and the follow-up measurements at six and 12 months.

It was decided to use these mixed recruitment strategies because previous studies by the Department of Health Promotion at Maastricht University showed that these strategies are sufficient to reach many smokers who are representative of the Dutch population of smokers and could also help to reach a sufficient number of LSES smokers (Smit et al., 2010, Te Poel et al., 2009).

\section{Randomization}

Smokers interested in quitting within the following six months were invited to visit the intervention website and could sign up with their own username and password. After signing up for participation, giving online informed consent and passing through the inclusion criteria, respondents were allocated to one of the three conditions of the program. Randomization was performed automatically by Tailor Builder computer software (OSE, Sittard, the Netherlands). This software was especially developed for the execution of web-based computer-tailored programs (OSE, 2011).

\section{Study course}

The course of the study is described briefly below. The main elements of the intervention are outlined in the Materials section.

The Tailor Builder software randomly assigned respondents either to one of the intervention groups (text- vs. video-tailored feedback) or to the control group (non-tailored 
generic advice). Respondents were not informed of the group to which they were allocated (see also Figure 1). The video-based condition used the same computer-tailored messages as the text-based condition. Respondents were asked to answer three questions concerning the inclusion criteria (18 years or older, smoker, intention to quit within a year or earlier). Respondents who did not meet the inclusion criteria were subsequently excluded from the study and received a message explaining why they could not participate in the study.

All other respondents were assigned to one of the three conditions, continued with the program and were asked to fill out the baseline questionnaire. At the end of the baseline questionnaire, respondents were asked to fill in whether they had plans to quit within the following month. Depending on their answer, respondents were allocated to one of two possible routings of the program.

Respondents with the intention to quit within the following month followed route 1 (detailed description of route 1 , see Materials section). Respondents were asked to choose a quit date and were invited seven days before their quit date to participate in the second session of the program. Respondents subsequently received an invitation for the third session three days after their quit date. Additionally, respondents received an invitation for the fourth, fifth and sixth feedback sessions two, four and eight weeks after their chosen quit-date.

Respondents not intending to quit within the next month followed route 2 (for details see Figure 2). One month after the baseline questionnaire respondents were invited by the program to follow the second session. At session two they were asked again to indicate their intention to quit within the following month. Respondents prepared to quit were subsequently directed to route 1 , as described above. Respondents not prepared to quit received their last invitation for the third session one month later.

Six and 12 months after filling out the baseline questionnaire, all respondents of the three conditions were asked to complete the follow-up measurements. In the e-health programs, invitations for the feedback sessions were sent by e-mail. Next, respondents received a personalised summary report by e-mail after each feedback session. Respondents in the text condition had the opportunity to reread and print the tailored text messages. Respondents in the video condition were able to watch the tailored video messages again. Respondents in the control condition were not able to reread the short piece of generic text advice. The average time of the different intervention sessions was about 20 minutes. 


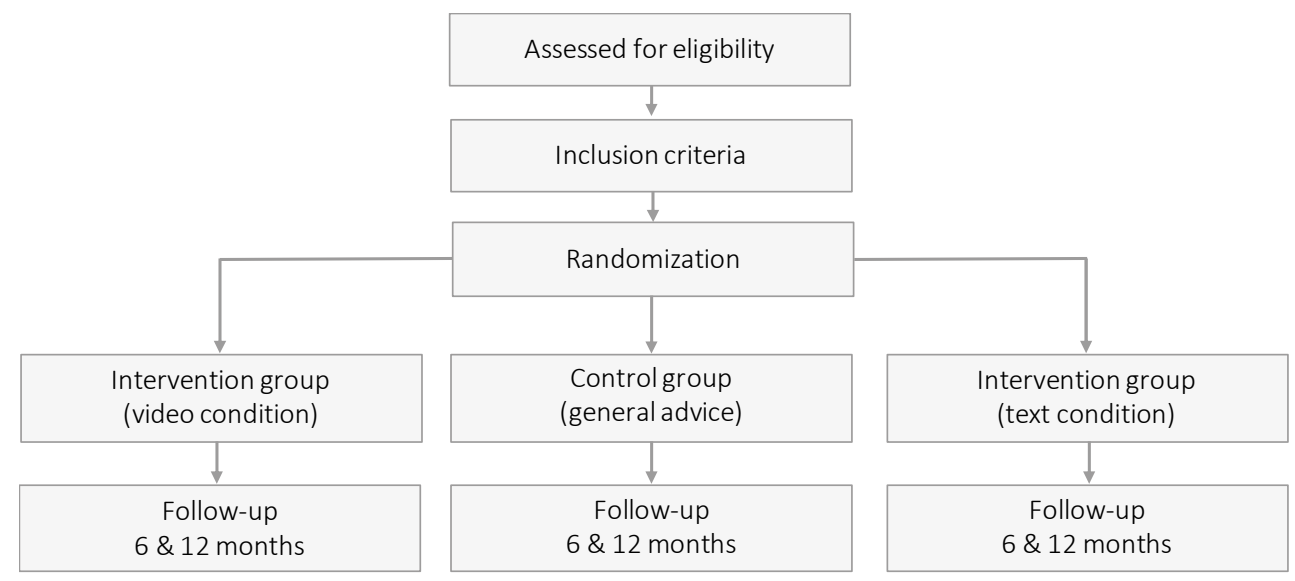

Figure 1 Flowchart of the randomized controlled trial

\section{Materials}

\section{Questionnaires}

In all three conditions respondents were asked to fill in the baseline questionnaire. The baseline questionnaire was based on the I-Change model (De Vries and Brug, 1999) and has previously been used in other studies that assessed the impact of computer tailoring on lifestyle changes, like smoking (Smit et al., 2010, Te Poel et al., 2009). The baseline questionnaire consisted of questions on smoking behaviour, smoking-related beliefs, social influence, self-efficacy to quit, preparatory planning and stage of change (Elfeddali et al., 2012c, Hughes et al., 2004, Te Poel et al., 2009). Furthermore, existing scales were used to measure cognitive processing (Cacioppo and Petty, 1982) and depressive complaints (Cole et al., 2004). The follow-up questionnaires at six and 12 months assessed smoking behaviour (Hughes et al., 2004), stage of change (Te Poel et al., 2009), quality of life (Qol) and health-care costs (EuroQol, 1990).

Social demographic information: Variables assessed were: age, gender, marital status, religious background, ethnic background, education level, current work status, income level.

Health status: Occurrence of smoking-related diseases was measured by four questions on a dichotomous scale (Do you suffer from chronic respiratory disease, cancer, diabetes or cardiovascular disease?; 0 = no; 1 =yes; index: 0 = no disease to 4 = occurrence of four diseases) (Te Poel et al., 2009).

Level of depression: Level of depression was measured by the abbreviated CES-D by four items (e.g. During the past week I felt depressed; 1 = rarely or none of the time (less than one day); 2 = some or a little of the time (one to two days); 3 = occasionally or a moderate amount of time (three to four days); 4 = most or all of the time (five to seven days) (Cole et al., 2004). 
Cognitive processing: Cognitive processing was measured by six items on a five-point scale of the Heuristic Systematic Processing Questionnaire (e.g. I like tasks where I do not have to think much; 1 = I totally disagree; 2 = I disagree; 3 = I do not know; 4 = I agree; $5=$ I totally agree) (Cacioppo and Petty, 1982).

Media preference style: Media preference style was assessed by a six-item questionnaire. The questionnaire which was developed by the research team assessed whether respondents preferred the visual or auditory channel in order to understand and remember new information (e.g. I can understand new information better if 1 = I can read it; $2=$ the information is reported by a person in a film; I remember information better if $1=\mid$ can read it; 2 = the information is reported by a person in a film).

Level of addiction: Level of addiction was measured by six items, asking respondents how many cigarettes/shags they smoked per day, at which time points and whether they had difficulties not smoking in smoke-free places. The answers to these six questions were converted into an overall score, ranging from one to 10 (Heatherton et al., 1991).

Smoking behavior: Smoking behaviour was measured by asking respondents how many cigarettes/shags they smoked per day. Smoking behaviour was converted into an overall score (number of cigarettes a day) (De Vries et al., 2008).

Habit: Smoking habit was assessed by an abbreviated version of the Verplanken and Orbell's Self-Reported Habit Index of six items with which respondents could agree or disagree on a five-point scale (e.g. Smoking is something which I do automatically; 1 = I totally disagree; 2 = I disagree; 3 = I do not know; 4 = I agree; 5 = | totally agree) (Verplanken and Orbell, 2003).

Attitude: Attitude towards the rational and emotional pros and cons of smoking was measured by 12 items with which respondents could agree or disagree on a five-point scale (e.g. When I do not smoke, my condition improves; $1=1$ totally disagree; $2=1$ disagree; $3=1$ do not know; 4 = I agree; 5 = I totally agree) (De Vries et al., 2008, Dijkstra et al. 1996, Te Poel et al., 2009).

Social influence: Social influence was measured by two existing scales; a social support scale and a social modelling scale (De Vries et al., 2008). Two items measured whether people in the respondents' environment smoked (respectively partners and people in the direct environment (Does your partner smoke?; 0 = no; 1 = yes; 9 = not applicable; how many of the people in your environment smoke?; 1 = none of them; 2 = the minority; 3 = half of them; 4 = the majority; 5 = all of them; 9 = not applicable). The second scale assessed whether the person received social support in favour of quitting or against quitting on a five-point scale (My partner/ people in my environment; 1 = do not support me; 2 = support me a bit; 3 = support me; 4 = support me a lot; 9 = not applicable) (De Vries et al., 2008).

Self-efficacy: Self-efficacy was measured by nine items on a five-point scale (e.g. Do you think you will manage not to smoke when you drink a cup of coffee?; 1 = definitely not; 2 = probably not; 3 = maybe yes; 4 = probably yes; 5 = definitely yes) (Dijkstra et al., 2000b). 
Action plans: Action plans were assessed by five items. Respondents had to indicate on a five-point scale whether they planned to execute different preparatory plans for their quit attempt (e.g. removing ashtrays, asking guests not to smoke and thinking about difficult situations they might encounter after quitting; 1 = surely not $-5=$ surely yes) (De Vries et al., 2008).

Coping plans: Coping plans were assessed by nine items. Respondents had to indicate whether they had made any plans to prevent relapse in difficult situations (e.g. plans how to cope with withdrawal symptoms, how to cope with negative mood and how to cope with high risk situation such as e.g. parties or being with friends; $0=$ no; $1=$ yes) (Hoving et al., 2006b).

Readiness to quit smoking: Readiness to quit smoking was measured by one item assessing whether the respondent intended to quit smoking on a six-item scale ( $1=$ yes, within the following month; 2 = yes, within one to three months; 3 =yes, within four to six months; 4 =yes, within one year; 5 =yes, within one and five years; $6=$ yes, but not within the following five years) (De Vries et al., 2008, Dijkstra et al., 1996).

\section{Intervention}

\section{Intervention elements of the two e-health programs}

The theoretical rationale behind computer-tailoring is to make the information that the user receives as personally relevant as possible. According to theories of information processing, e.g. the Elaboration Likelihood Model, people pay more attention to personally relevant information, thoughtfully consider it and appreciate it more than non-personally relevant information (De Vries et al., 2008). Research has furthermore shown that information which is attended to and thoughtfully considered is more likely to influence a person's beliefs and behaviours (Kreuter et al., 1999a).

The tailored program consisted of a screening instrument for smoking behaviour, and a feedback library with all pre-written feedback messages and tailoring algorithms. The tailoring algorithms linked up the specific answers of the respondent with the relevant corresponding health message in the feedback library. The screening instrument consisted of different questionnaires, which were developed to assess the smoking behaviour itself, the perception of the smoking behaviour and determinants such as attitude, social norms and selfefficacy towards smoking. After completion of the questionnaires, automatized feedback was generated and provided based on the specific answers the respondent gave. Feedback was provided on item level (e.g. for each coping plan). The video-based condition used exactly the same tailored messages as the text-based condition. The video had a new reading format with five different adults delivering the messages (two males, three females) who were chosen as a result of screen tests. In the context of learning, research has shown that students learn significantly more and are more motivated when information is presented through multiple visual agents of different gender and race. This can be explained by the fact that different 
information might be better understood and separated if it is delivered by separate agents (Baylor, 2009). With regard to the selection of the speakers we therefore used a mix of adults in terms of age, gender and ethnicity. Research has also shown that individuals are more influenced by visual agents who look similar to themselves regarding appearance-related characteristics (Bailenson et al., 2008). We therefore tried to choose normal adults with normal everyday clothes with which smokers can easily identify. We furthermore aimed to exclude adults with strong dialects in order to avoid distraction or lack of comprehension. Respondents in the two experimental conditions received personalised information in several feedback sessions, depending on a smoker's readiness to plan a quit date within the following four weeks (see previously described study course). The feedback messages of the program aimed to increase the respondents' positive attitude toward quitting, to increase the respondents' motivation to make a quit attempt and to support the respondents during the period after their quit attempt. Multiple feedback moments were included in the program because they have been shown to be more effective in encouraging health behaviour than a single feedback moment (Smeets et al., 2008).

\section{Baseline computer-tailored session}

The baseline computer-tailored session consisted of a shortened version of a previously tested computer-tailored program that was found to be more effective in increasing smoking cessation than the provision of generic advice and information (De Vries et al., 2008, Te Poel et al., 2009). The baseline session was intended to increase the respondent motivation to quit smoking and to encourage the respondents to set a quit date in the next few months. Respondents received tailored feedback on the perceived advantages and disadvantages of quitting. Tailored feedback was offered with regard to the different pros of quitting (e.g. decreased likelihood of diseases) in order to convince the respondents of the advantages of quitting. Tailored feedback further addressed the disadvantages of quitting (e.g. withdrawal symptoms) in order to combat possible negative existing beliefs. With respect to the respondent's perceived social support, tailored feedback dealt with how to deal with smokers in their environment (e.g. asking them not to smoke in their environment in order to avoid a relapse). Furthermore, respondents received feedback on their perceived self-efficacy, the importance of self-efficacy for successful quitting and tips for increasing self-efficacy. Lastly, information was provided on how to plan quitting (e.g. planning a quit date and using smoking cessation aids). Depending on the readiness to quit within one month or not, respondents received feedback in one of the two routings that are explained in detail below. 


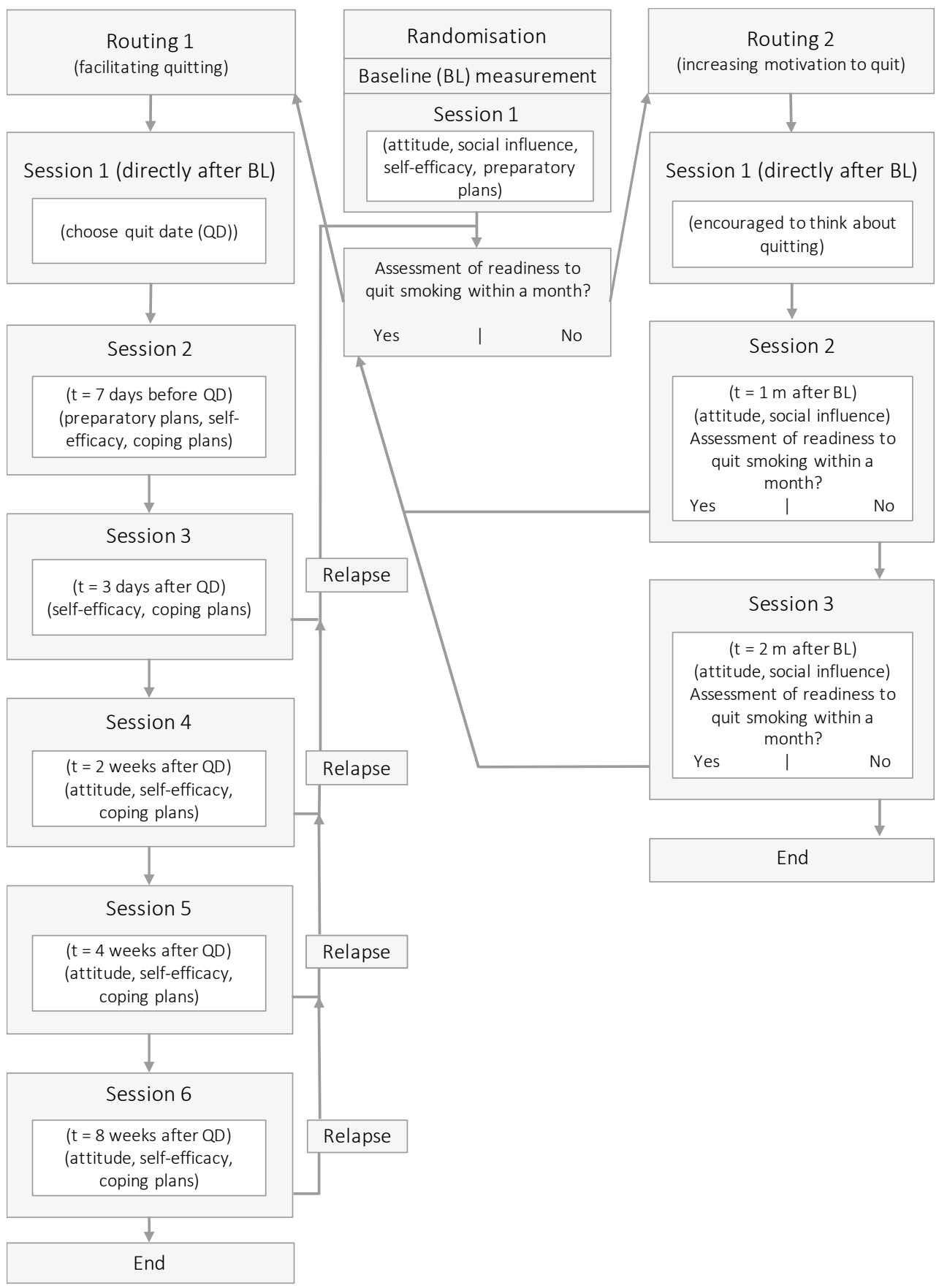

Figure 2 Flowchart of the intervention 


\section{Routing 1}

Respondents who were ready to quit in the next month were directed into routing 1 . After the baseline questionnaire, respondents in session one were asked to set a quit date. Respondents were requested to choose a quit date between eight days and one month from baseline. At the end of the first session, smokers were informed by e-mail that they would be invited for the next session of the program one week before their quit date in order to receive new support and help in preparing their quit attempt in the following days.

One week before their quit date respondents were invited by e-mail to follow the next session of the program. The second session was aimed at preparing the smoker to quit successfully and to be prepared for the difficulties that might arise after quitting. Respondents were first asked to fill out a short questionnaire on self-efficacy and action planning items. Several studies identified a low level of self-efficacy as a good predictor of smoking relapse (Elfeddali et al., 2012b, Gwaltney et al., 2005, Gwaltney et al., 2009). Therefore, respondents received feedback on how to increase self-efficacy. Tailored feedback on self-efficacy was provided in order to help the respondent to gain confidence to quit and to help him/her to deal with the possible difficulties and negative consequences of their quit attempt. Next, respondents were encouraged to make preparatory plans for their quit attempt. Tailored feedback addressed the advantages of making plans in advance and respondents were furthermore stimulated to think about concrete plans (e.g. removing ashtrays, asking guests not to smoke and thinking about difficult situations they might encounter after quitting). Lack of preparatory planning has been shown to be a good predictor of smoking relapse and preparing for quitting has been shown to increase smoking cessation (Te Poel et al., 2009). The second session not only aimed at preparing the smoker to quit successfully (by encouraging preparatory planning) but also to prepare the smoker to cope with the challenges/difficulties that might arise after quitting. This so-called coping planning has been shown to be a helpful strategy for dealing with high-risk situations (Gollwitzer, 1999). To encourage coping planning, respondents were first asked to choose from a list of 11 risk situations (e.g. I find it difficult not to smoke if I am angry, I find it difficult not to smoke when I see another person enjoying a cigarette) the three most personally relevant high-risk situations. Next, respondents were asked to indicate whether they had already thought about how to deal with these high-risk situations. If they had not thought about coping plans, respondents were instructed to formulate specific coping plans for their personal high-risk situations. Examples were given of how to make concrete plans (e.g. Next time you feel angry, instead of smoking a cigarette, go outside and have a walk).

Session three took place three days after the quit date of the respondent. At that time, respondents were invited by e-mail to turn to the computer-tailored program again and to fill out a brief questionnaire on self-efficacy and coping planning items. The third session was aimed at encouraging the respondent again to think about possible coping plans in order to 
deal with high-risk smoking-related situations. The same strategy was used as in session two. Respondents were also asked about their smoking behaviour since their quit date. In cases of lapse/relapse the respondent was told that smoking cessation is a process in which lapses may occur and that they can be used positively in continuing the quit attempt. Respondents were also encouraged and invited to continue their quit attempt or to plan a new quit date. This strategy derived from a computer-tailored relapse prevention preprogram that has shown to be effective in preventing relapse (Elfeddali et al., 2012b).

Two weeks after the quit date respondents were invited for session four. Respondents were instructed to fill out again a brief questionnaire on self-efficacy and coping planning items. The same procedure was used as in session two and three of the program. Furthermore, pros and cons of quitting were assessed again as in session one. This was done in order to encourage the respondents to rethink about the pros and cons of smoking and quitting that they perceived and to assess whether respondents had acquired possible new pros of quitting since the first session of the program.

In sessions five and six, after four and eight weeks, a similar strategy was used to that in session four. During the last three sessions, respondents could still choose to receive tailored feedback on different items (such as how to cope with negative moods or how to cope with high-risk situations). This option was provided since we expected them to encounter different problems throughout their quit attempt. In all these sessions, smokers who had reverted to smoking were invited and encouraged to restart their quit attempt.

\section{Routing 2}

Respondents who were not ready to quit in the next month followed routing 2. At the end of session one, that followed immediately after the baseline session, respondents were encouraged to use the next month to reflect on their quitting intention and the tailored information they received during session one. After one month these smokers received an email with an invitation for the second session. In session two, the respondent was asked about his/her smoking behaviour. Subsequently respondents again received feedback on the perceived advantages and disadvantages of smoking and quitting and how best to obtain social support from their environment. At the end of the session smokers were asked to indicate their readiness to quit in the next month. Respondents who were ready to quit in the next month and ready to plan a quit date were sent to routing 1 . Respondents not intending to quit in the next month, were informed that they would receive an invitation for session three one month later to reassess their smoking behaviour and their motivation to quit.

As in session two, respondents received in session three tailored feedback on the perceived advantages and disadvantages of smoking and quitting and how best to obtain social support from others. Smokers were again encouraged to quit and were asked at the end of the session to indicate their intention to quit in the next month. Respondents ready to quit within 
the next month and ready to plan a quit date were sent to routing 1 . Smokers not prepared to plan a quit date within a month received an empathic message, indicating that it was respected that they were not ready to quit smoking and that we would therefore send no further e-mails at this stage.

Finally, at the end of the program, all respondents were informed that they would receive an invitation (by e-mail) after six and 12 months to fill out a brief follow-up questionnaire about their smoking behaviour and a process evaluation questionnaire on the programs after six months.

\section{Control group}

The control group received brief generic text advice about smoking cessation. The content of the generic text was similar to the experimental conditions. Although brief advice was given on how to quit successfully, the generic text advice was not tailored to personal factors and respondents in the intervention group all received the same information. Furthermore, respondents were not provided by e-mail with an overview of the brief non-tailored text advice.

\section{Outcomes and biochemical validation}

Primary outcome measurement

Primary outcome measures of this study measured at six and 12 months after baseline were seven-day point prevalence abstinence from smoking, continued abstinence and prolonged abstinence. Seven-day point prevalence was defined as not having smoked during the last seven days (measured from follow-up). Continued abstinence was defined as not having smoked since the last quit date, whereas prolonged abstinence took into account a grace period of two weeks (Hughes et al., 2004, Mudde et al., 2006). During this grace period reinitiating of smoking behaviour after the quit date was not defined as a relapse. All outcome variables were measured according to the definitions in Hughes et al. (Hughes et al., 2004, Mudde et al., 2006). Smoking during the last seven days and after the personal quit date was coded as relapse (0), whereas non-smoking during the last seven days and since the quit date was coded as abstinence (1).

\section{Secondary outcome measures}

Secondary outcome variables measured at six and 12 months after baseline included 24-hour point prevalence abstinence (having smoked during the last 24 hours; $1=$ no; $0=$ yes), having made a serious quit attempt (not having smoked for at least 24 hours; $1=$ no; $0=$ yes) and changes in smoking behaviour since baseline measurements (Mudde et al., 2006). All respondents were further asked to fill out a process evaluation questionnaire at the six-month follow-up to evaluate their experience of the program. 


\section{Biochemical validation}

At the last follow-up measurement (12 months after baseline) self-reports regarding cessation will be biochemically validated by means of a cotinine test. We aim to validate self-reports randomly in a subsample of respondents who have indicated abstention from smoking. At least $50 \%$ of this sample will be invited to participate. We aim to conduct the biochemical validation within one week after the 12-month follow-up measurement. Respondents reporting abstention at the 12-month follow-up will be approached by a research assistant to make an appointment for the cotinine test (e.g. at home or at work). Saliva of the respondents will be collected with a swab stick and will be applied to a test strip. Respondents who decline to undergo the test will be asked by the research assistant for the reason(s) why.

\section{Statistical analysis}

Sample size calculation

Sample size calculations were based on the ability to detect $10 \%$ differences between the three conditions with a power of 0.80 and an alpha of $5 \%$. To calculate the sample size, seven-day point prevalence abstinence at the six-and 12-month follow-up was taken as the base since it is considered to be the most sensitive and valid measure of smoking cessation (Velicer and Prochaska, 2004).

Power calculations were made separately for LSES and HSES groups. For the LSES group we expected that the video condition will result in an $18 \%$ quit rate, and the text condition in an $8 \%$ quit rate, requiring 176 respondents in both conditions. For the HSES group we expected quit rates of $22 \%$ in the text-based condition and $12 \%$ in the video-based condition, requiring 220 smokers for both conditions.

\section{Attrition prevention}

Different strategies have been applied in order to prevent high attrition rates. First, respondents completing all questionnaires are eligible to win a price of $€ 100$. Second, the follow-up questionnaires at six- and 12-months are brief, only aiming to assess behaviour, readiness to quit smoking, since this strategy has been found to increase participation rates by approximately $8 \%$ to $10 \%$. Respondents who do not respond to the follow-up measurement at six- and 12-months receive two reminders inviting them again to fill in the follow-up measurements. Respondents who still do not react will receive an invitation by e-mail to briefly indicate their current smoking status by indicating whether they have smoked during the last 24 hours, in the last week or in the last months, since this strategy was shown in another study to result in an additional response rate of $8 \%$ to $10 \%$. 


\section{Analysis}

We will conduct logistic regression analysis at six- and 12-months to assess program effects on the main outcome variables. We will further perform linear regression analysis and covariance analysis to assess effects on secondary outcome variables at six- and 12-months. We will correct for baseline factors (e.g. demographics, smoking behaviour, attitude, intention) in both types of regression analyses by adding these variables as possible confounders. Interaction effects of experimental condition (text vs. video) and SES will be explored by moderation in the logistic regression analysis since we expect an interaction effect between SES and condition.

\section{Additional studies}

Additional to the effect study we will conduct two other studies in the same trial, a process evaluation and an economic evaluation, which are described briefly below.

\section{Process evaluation}

We will conduct a process evaluation to assess the respondents' reactions to the program. Furthermore we will assess differences in evaluation between LSES and HSES smokers. The questionnaire of the process evaluation is based on previously used questionnaires (De Vries et al., 2008, Smeets et al., 2008) and assesses information appraisal of the tailored advice. The concepts attention, comprehension and appreciation of the tailored advice are measured on a five-point scale. Attention is measured by assessing whether the respondents felt that the messages attracted their attention and retained it. Comprehension is measured by asking whether they understood the messages and whether they found them difficult or encountered difficulties with words or passages. Appreciation of the advice is measured by asking whether the respondents liked the messages and whether they found the message personally relevant.

\section{Economic evaluation}

The economic evaluation involves a combination of a cost-effectiveness analysis (CEA) and a cost-utility analysis (CUA) to analyse whether the e-health program is preferable in terms of cost, effects and utilities from a societal perspective. In the CEA, the incremental costeffectiveness ratio (ICER) is expressed as the incremental costs per additional quitter (measured as 12 months point prevalence abstinence). In the CUA, the outcome measure is quality-adjusted life years (QALYS), which is based on the EuroQol utility score (EuroQol, 1990). 


\section{Discussion}

The paper describes two multiple computer-tailored smoking cessation programs and the design of an efficacy study that will be conducted to test the effects of the programs on smoking cessation. The computer-tailored e-health approaches are currently tested by Dutch smokers who are motivated to stop smoking. The tailored messages are the same in both conditions; the two conditions only differ in the mode of delivery. One experimental condition uses video-driven messages whereas the other condition uses text-driven messages. The two experimental conditions (video vs. text) will be compared with each other and with a control condition which receives general smoking cessation advice. Furthermore, the effects of the two different multiple tailored smoking cessation programs will be tested among smokers with a lower and higher socioeconomic background.

The present study has several strengths. First, it addresses the efficacy of two computer-tailored programs by means of a strong experimental design. A randomized control trial was used to exclude possible biases with regard to assignment of respondents to the different conditions. Allocation of the different conditions was performed automatically with the Tailor Builder computer software.

Second, the intervention is one of the first programs to test video-based messages as a potential new communication strategy for LSES smokers. So far computer-tailored smoking cessation interventions have tended to rely on text-based messages. Several studies however suggest that videos may be more effective in attracting attention and stimulating comprehension, especially in LSES smokers (Nielsen and Sheppard, 1988).

Third, an important strength of our smoking cessation programs concerns the inclusion of multiple tailored feedback moments. Tailored information has been shown to be more likely to be read, remembered and considered personally relevant. Our study made use of a multiple computer-tailored smoking cessation intervention with different feedback moments. Although previous programs did focus on smoking cessation tailored messages, few of them provided smokers with multiple tailored feedback moments.

Finally, one strategy the study used to collect respondents was via the GP setting. The GP is considered as an important access point to many smokers (Van den Berg, 2003). Advice from GPs is an effective way to help smokers to quit and is seen as valuable by the Dutch patient (Wensing et al., 2002).

There may be limitations to the study. First, the multiple tailoring e-health programs could be too intensive and therefore lead to an increased drop-out rate. Furthermore, the program is only available on the Internet, so smokers with no Internet access were excluded from the study. Although $90 \%$ of Dutch households are equipped with Internet access this could still result in selection bias (CBS, 2011a). 


\section{Conclusion}

A description was provided of the e-health smoking cessation intervention SteunbijStoppen.nl, which was developed especially to attract smokers with a LSES. The paper also explained the classification of the study into efficacy and cost-effectiveness of the two programs compared with a control condition and user evaluation. The study results reveal different working mechanisms of multiple computer-tailored smoking cessation interventions. The results will help us to gain more insight into effective strategies to target different subgroups and especially smokers with a LSES. Finally, the results of the study will contribute to the development of future smoking cessation e-health programs. The different behavioural effects of the Steunbijstoppen.nl intervention will be published in other papers. 



\section{CHAPTER 3}

How to encourage smokers to participate in web-based computertailored smoking cessation programs: a comparison of different recruitment strategies

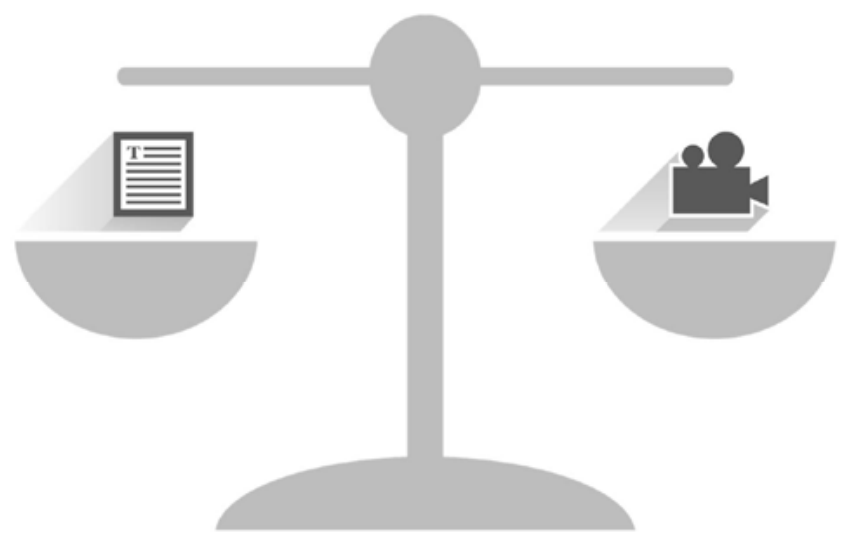

Published as:

Stanczyk, N. E., Bolman, C., Smit, E. S., Candel, M. J. J. M., Muris, J. W. M. \& de Vries, H. (2013). How to encourage smokers to participate in web-based computer-tailored smoking cessation programs: a comparison of different recruitment strategies. Health Education Research, cyt104v1-cyt104. 


\begin{abstract}
Objective: The reach, retention, and costs of four strategies aimed to recruit smokers for participation in a computer-tailored smoking cessation intervention was explored.

Methods: The study was part of a randomized controlled trial whereby 832 respondents were randomised to three conditions. Smokers were invited by general practitioners (GP), newspapers, Internet and other strategies (i.e. mailing organizations) to take part. ANOVA's /Chi-square tests explored sample differences. Logistic regression analyses investigated differences between the samples regarding retention and smoking behaviour.

Results: Smokers recruited via GPs $(n=144)$ had a lower educational level and suffered more from chronic obstructive pulmonary disease compared to respondents recruited via Internet $(\mathrm{n}=307)\left(\chi^{2}=11.55, \mathrm{df}=3, p=.009\right)$. Less motivated respondents, recruited by GPs were more likely to return to study compared to the less motivated respondents recruited by 'other recruitment' strategies $\left(\chi^{2}=6.42, \mathrm{df}=3, p=.09\right)$. Highly addicted respondents recruited from newspapers ( $n=213$ ) were less likely to make a quit attempt compared to highly addicted respondents recruited by GPs $(\mathrm{OR}=0.33, p=.04)$. Females from newspapers were less likely to remain abstinent compared to the GP sample $(\mathrm{OR}=0.34, p=.005)$. Recruitment via GPS showed highest costs.
\end{abstract}

Conclusion: Recruitment strategy influenced the type of smokers. Group differences were associated with different patterns of quitting.

Keywords: smoking cessation, recruitment strategies, computer tailoring, web-based interventions 


\section{Introduction}

Although web-based computer-tailored interventions have been developed to aid smokers in quitting (Civljak et al., 2010, Dijkstra et al., 1998a, Elfeddali et al., 2012a, Smit et al., 2012, Te Poel, et al., 2009), reaching and recruiting smokers to participate and adhere to these interventions is a challenge (McDonald, 1999). It might become especially challenging to reach the remaining segment of the smoking population, namely those who are least ready to quit (Cavelaars et al., 2000, Jha et al., 2006, Lopez et al., 1994). Although past research indicates that web-based smoking cessation interventions are potentially effective (Hoving et al., 2010, Krebs et al., 2010, Myung et al., 2009, Rooke et al., 2010, Shahab and McEwen, 2009), less is known about strategies to encourage smokers to enrol in these programs and about their adherence to these programs (Richardson et al., 2013).

Even though web-based interventions are believed to be able to reach a large number of people (Balmford et al., 2008, Griffiths et al., 2006), research has shown that only a small part of the smoking population is actually reached by these interventions (Evers et al., 2005). Mostly higher educated respondents with a healthy lifestyle pattern make use of web-based lifestyle interventions (Brouwer et al., 2011, Brouwer et al., 2010, Crutzen, 2009a). Moreover, people with a lower education level may less likely to be reached by online interventions (Lorence and Park, 2008, Van't Riet et al., 2010). As lower educated smokers show high addiction levels and experience the most problems in quitting (Fidler et al., 2008, Kotz and West, 2009, Nagelhout et al., 2012, Peters et al., 2005), it is important to obtain more insight in how different educational groups and especially lower educated smokers can be best recruited to participate in these interventions. Past research has already indicated that computer-tailored smoking cessation interventions have the potential to positively influence lower educated groups (Stanczyk, et al., 2013). Investigating user's characteristics and their reach for participation in smoking cessation interventions can therefore be helpful in optimizing ways of disseminating smoking cessation interventions among different educational groups.

Strategies to recruit smokers into smoking cessation programs often include face-toface referral (e.g. by general practitioners (GPs)), media campaigns, Internet campaigns and other methods such as postal or e-mail invitations. Past research has already distinguished between those seeking information regarding quitting, who are often highly motivated to quit versus a cold-contacted group, recruited via an unsolicited form, who are on average often less motivated to quit (Balmford et al., 2013). A recent study has already indicated that large media campaigns were an effective recruitment tool, but are associated with high costs (Czarnecki et al., 2010). Internet campaigns, in contrast, have the potential to reach a large audience against low costs, but its actual reach among different educational groups is not yet clear (Swartz et al., 2006). In contrast, recruitment via mass media channels has shown to be effective in 
recruiting numerous smokers from different educational levels (Graham et al., 2008) and is also associated with lower retention and success rates compared with recruitment via GPS (Smit et al., 2011). Recruitment via GPs, on the other hand, was assumed to be successful in reaching lower educated smokers in particular, although the net effect in quitters was still in favour of the mass media approach (Hoving et al., 2007, Smit et al., 2011). To optimise the public health impact of smoking cessation interventions, it is therefore, important to examine which recruitment strategy is most effective, and to further explore effects of additional strategies and whether or not these effects differ depending on the educational level of respondents.

This study explored the reach, retention and costs of four different strategies aimed to recruit smokers for participation in a web-based computer-tailored smoking cessation intervention. First, we examined how many smokers were reached with each strategy and whether the recruitment strategies (recruitment via GPs, newspapers, Internet and other strategies (e.g. e-mailing to companies, referrals)) resulted in samples consisting of different types of smokers. Second, we investigated whether possible differences existed between the recruited samples regarding retention, quit attempts and smoking abstinence, measured six months after baseline. With respect to the first two goals, we were especially interested in smokers with lower educations since they are most 'in need' and hard to reach (Nagelhout et al., 2012, Peters et al., 2005). Finally, different costs of the recruitment strategies were examined in relation to recruitment success to determine the costs per recruited respondent, per respondent who made a quit attempt and per abstinent respondent.

\section{Methods}

\section{Study design}

The study was part of a randomized controlled trial (Dutch Trial Register (NTR3102)) (Stanczyk et al., 2011) in which three conditions were compared. Respondents were randomized to one of the two experimental conditions (video- or text-based computer-tailored intervention) or the control condition (respondents received a short general text advice after baseline on smoking cessation). Respondents were not informed which group they had been allocated and had no information about the content of the other experimental conditions. In the text condition, respondents were presented multiple text-based computer-tailored messages on smoking cessation. In the video condition, the same tailored messages were used but were translated into video-driven messages (without graphics/animations). 
This study was submitted for approval to the Medical Research Ethics Committee (MREC) of Atrium Medical Centre Heerlen. The MREC decided that no MREC approval was necessary because respondents were not obliged to engage in medical acts. The study was conducted according to the American Psychological Association (APA) principles (American Psychological Association, 2002).

\section{Target population and inclusion criteria}

Respondents were recruited in the Netherlands from December 2010 until November 2011. Daily smokers of 18 years and older, who were motivated to quit within the following six months and had access to the Internet were eligible for study participation.

\section{Recruitment methods and related costs}

Respondents were recruited by several strategies. The goal of the recruitment was to encourage smokers to visit the Dutch Intervention website: www.steunbijstoppen.nl (Steunbijstoppen.nl, 2012) and to register for participation.

GP practice: Respondents were recruited through GPs. About 2,000 GPs in the Netherlands were invited by mail, whereof about 150 GP practices agreed to refer a minimum of 20 smoking patients, who met the inclusion criteria, to the intervention website. In the Netherlands, smoking cessation treatment recommendations for GPs are to ask patients with smoking-related illnesses about their smoking behaviour, to give stop smoking advice and to refer the patient to stop smoking treatment programmes (Chavannes et al., 2007). During our study, GP practices received recruitment materials, including a description of the project, business cards and flyers that they could give to eligible patients visiting their practice (instead of referring them to the usual channels). Their only task was to refer patients to the intervention website. GPs were not compensated for their time spent on recruiting smokers for our study. Since the GP practices often have a limited amount of time to ask patients about their smoking and do not reach all potential smokers, a mix of recruitment strategies was used. In this way, we assumed to reach a broader group of potential quitters and we were able to investigate whether different strategies attract similar or different samples of smokers.

Newspaper advertisement: Respondents were therefore also recruited by several paid advertising campaigns in national newspapers and different free insertions in local newspapers. Advertisings directly referred smokers to the intervention website, where they could receive information about the intervention and participation in the study.

Internet advertisement: Recruitment via the Internet consisted of paid advertisings on newspaper websites and free insertions on websites of national health funds (e.g. the Dutch Diabetes Foundation). These insertions were directly linked with the intervention website. This procedure enabled people to visit our intervention website for further information. 
Companies: Several companies recruited respondents. Targeted mailings were sent to approximately 33 different companies; companies were asked to bring the intervention to the attention of their employees by means of advertisements or announcements. The same as with the other recruitment strategies, advertisings referred employees to the intervention website.

Local free media: Respondents were also recruited through local free media (e.g. TV interviews and information on teletext).

Referral: It was also registered whether respondents received a referral of a friend or family member to visit the intervention website.

\section{Procedure}

Respondents were randomized into one of the three groups regardless of method of recruitment. After giving online informed consent to participate, respondents were invited to fill out the baseline questionnaire, assessing their demographics, smoking behaviour, beliefs about quitting, occurrence of different diseases and mode of recruitment. Depending on their readiness to quit, respondents in the two experimental conditions received tailored feedback over a period of one to three months whereas respondents in the control condition received general text advice about smoking cessation after baseline. Six months after baseline, all respondents were asked to complete the follow-up measurement. Several strategies were also applied to prevent attrition at follow-up (see Figure 1).

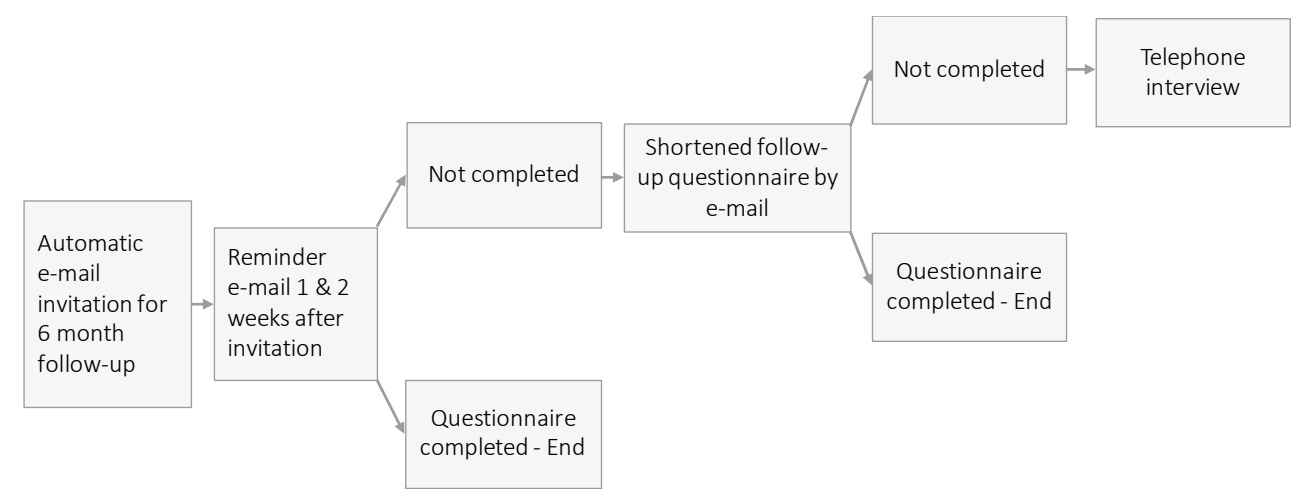

Figure 1 Procedure of reminder e-mails 


\section{Intervention}

The web-based multiple computer-tailored smoking cessation intervention was based on the I-Change model (ICM), a model integrating various social cognition models to explain and change health behaviours (De Vries et al., 2003). The model recognizes three phases for behavioural change: awareness, motivation and action. This implies that in order to motivate people to develop intentions to quit smoking, they need to become convinced of the pros and cons of the health behaviour (i.e. smoking cessation), need to perceive social support, and feel self-efficacious. In order to facilitate the translation of intentions into action, an individual is encouraged to make a specific action plan to prepare the new behaviour, to cope with challenges to prevent relapse, and to act on these plans (De Vries et al., 2013). The content of our intervention was partly based on earlier effective computer-tailored smoking cessation interventions (Smit et al., 2010, Smit et al., 2012, Te Poel et al., 2009). Depending on smokers' readiness to quit smoking within one month or not, respondents in the two experimental conditions (video vs. text computer tailoring) received personalised feedback during multiple computer-tailored sessions, using two separate routings. In routing 1, respondents motivated to quit within one month were asked to set a quit date and received support in preparing quitting and staying abstinent during the following three months. In routing 2 , respondents who were not motivated to quit within one month were invited to follow the next session one month after baseline. They then received feedback on advantages and disadvantages of smoking and quitting smoking and were encouraged to set a quit date. Smokers who were motivated to quit were directed to routing 1 , if not, smokers were invited to the next session (one month later) to again reassess their smoking behaviour (see Figure 2). The feedback sessions each took about 20 - 30 minutes. A detailed description of the intervention and its components is reported elsewhere (Stanczyk et al., 2011). 


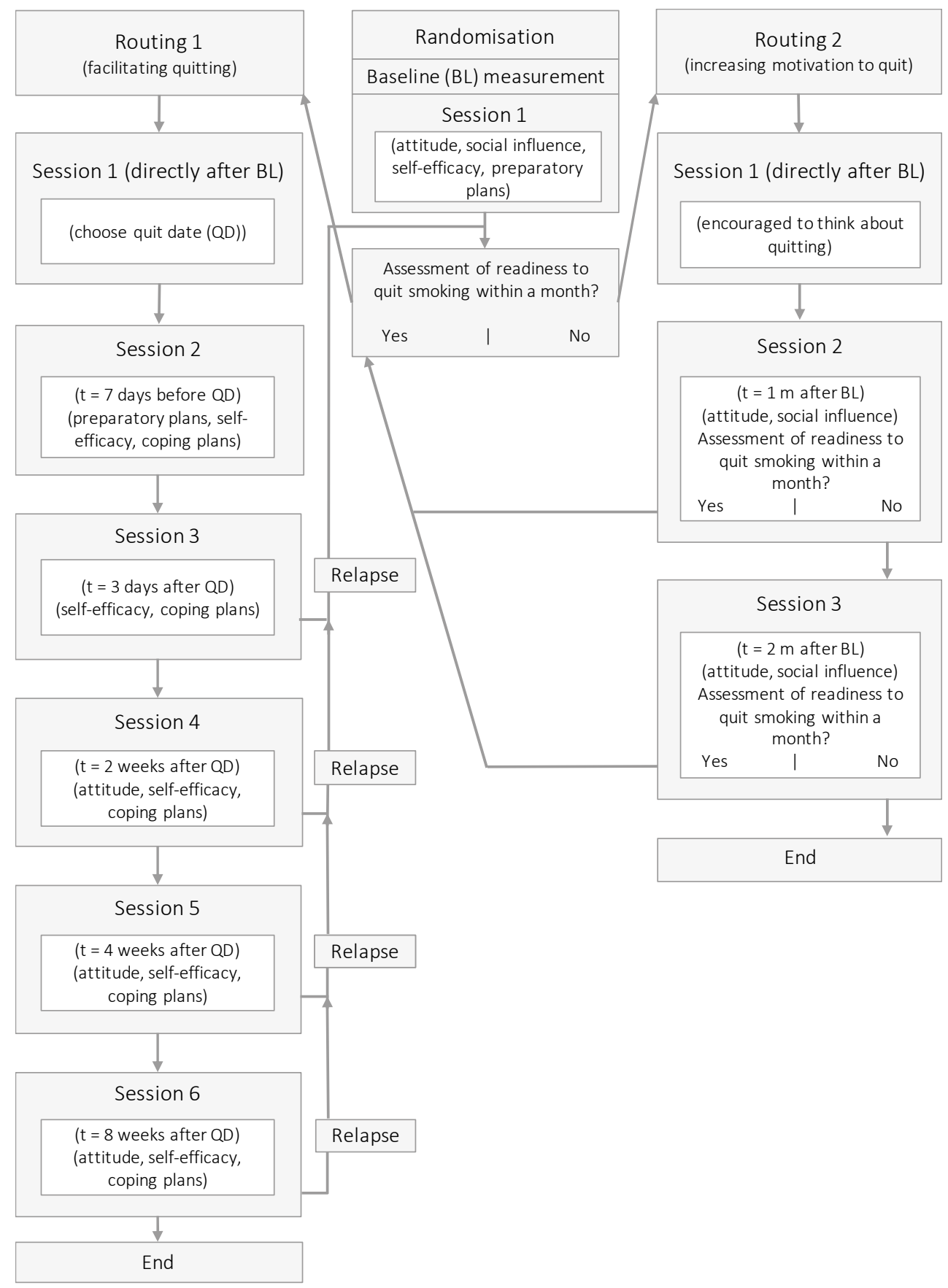

Figure 2 Intervention design 


\section{Questionnaire}

\section{Baseline measures}

The following demographic variables were measured at baseline: gender ( $0=$ male; $1=$ female $)$, educational level ( $3=$ high (higher vocational school or university level); 2 = intermediate (higher general secondary education, preparatory academic education, medium vocational school); 1 = low (primary, basic vocational, lower general school)) (CBS, 2012), nationality (0 = other nationality; 1 = Dutch nationality) and age.

Addiction level was assessed by six items using the Fagerström Test for Nicotine Dependence (FTND), asking respondents how many cigarettes they smoked per day, at which time points and whether they had difficulties not to smoke in smoke-free places. The answers were converted into an overall sum score ( 0 = not addicted; $10=$ highly addicted) (Heatherton et al., 1991).

Readiness to quit smoking was measured by one item asking respondents when they intended to quit smoking, resulting in five categories ( $5=$ yes, within the following month; $4=$ yes, within one to three months; 3 = yes, within four to six months; 2 = yes, within one year; 1 = yes, though not within a year, but within one and five years or later) (De Vries et al., 2008).

The occurrence of chronic obstructive pulmonary disease (COPD), cardiovascular disease, cancer, diabetes and asthma were assessed by four questions, asking respondents whether they suffered from these diseases ( $0=$ no; 1 = yes).

Mode of recruitment was measured by one item asking respondents how they learned about the intervention website, resulting in several categories: 1 = via GP; 2 = via newspaper; $3=$ via Internet; 4 = via companies; 5 = local free media and 6 = via a referral of a family member or friend. Recruitment categories $4(n=81), 5(n=14)$ and $6(n=73)$ were combined into one single category mentioned in the following part as 'other' strategies due to the fact that there were insufficient cases to analyse them separately.

Retention was defined as still being in the study after six months, measured by whether or not the respondent returned to fill out the six-month follow-up measurement $(0=$ not filled out; 1 = filled out).

Outcome measures at six-month follow-up

At the six-month follow-up questionnaire, seven-day point prevalence abstinence was assessed by asking respondents whether they had smoked one or more cigarettes during the last seven days ( 0 = yes; 1 = no) (Mudde et al., 2006). Respondents were also asked whether they had undertaken a serious quit attempt (defined as having refrained from smoking more than 24 hours) since baseline ( $1=$ yes; $0=$ no). 


\section{Analyses}

First, descriptive analyses were used to investigate baseline differences of the four samples. Analyses of variance were used to assess differences in continuous baseline variables. If the $F$ test showed a $p<.05$, the Tukey-HSD method was used for post-hoc pairwise comparisons. Chi-square tests were used to assess differences in categorical baseline variables. If the Chisquare test showed a $p<.05$, post-hoc pairwise comparisons with Bonferroni correction alpha $=.05 / 3=.017$ were used. Baseline differences were included as potential covariates in all analyses.

Next, logistic regression analyses using complete cases were conducted to assess whether the recruitment samples differed with regard to retention. Finally, logistic regression analyses were conducted to investigate whether mode of recruitment had an effect on the outcome measures. A top down procedure was used, beginning with the most extensive regression model, including recruitment mode, covariates (gender, education, level of addiction, age, occurrence of diseases, experimental condition and motivation to quit) and possible interactions between mode of recruitment and covariates. We first used the GP sample as the reference group and afterwards reran the same analyses, this time with the other three strategies, respectively, as the reference group to investigate possible differences between the other three strategies. Non-significant interaction effects were removed from the regression model. Interaction effects were regarded to be significant when $p<.10$; this was done in an effort to lower the risk of mistakenly overlooking a 'true' interaction effect (Marshall, 2007). In the case of a significant interaction effect, subgroup analyses were done. Main effects were considered to be significant when $p<.05$. To test the robustness of the results, a sensitivity analysis was conducted regarding all drop-outs as smokers.

All analyses were conducted with SPSS 19.0 (SPSS Inc., Chicago, IL, USA).

\section{Results}

\section{Sample characteristics related to recruitment strategy}

Figure 3 displays the flow of respondents. From the 1,016 potential respondents, 20 did not meet the inclusion criteria, 31 declined participation and 133 did not finish the baseline questionnaire and were therefore excluded from all further analysis. Thus, 832 respondents were randomized into the video condition $(n=270)$, the text condition $(n=272)$ and the control condition $(n=290)$. The baseline differences between the recruitment samples are presented in Table 1. The four recruitment strategies attracted somewhat different samples of smokers. We found differences in educational level $\left(\chi^{2}=24.41, \mathrm{df}=6, p=.000\right)$ between the samples, such that the GP recruitment yielded a larger proportion of lower educated respondents compared with the other three recruitment strategies. Furthermore, differences were found in the samples with regard to gender $\left(\chi^{2}=14.04, \mathrm{df}=3, p=.003\right)$. Internet advertisements 
yielded a larger proportion of female smokers compared with respondents recruited via 'other' strategies. In addition, there were differences in age $(F(3,828)=8.21, p=.000)$, such that those recruited from newspapers and GPs were older compared with the other two strategies.

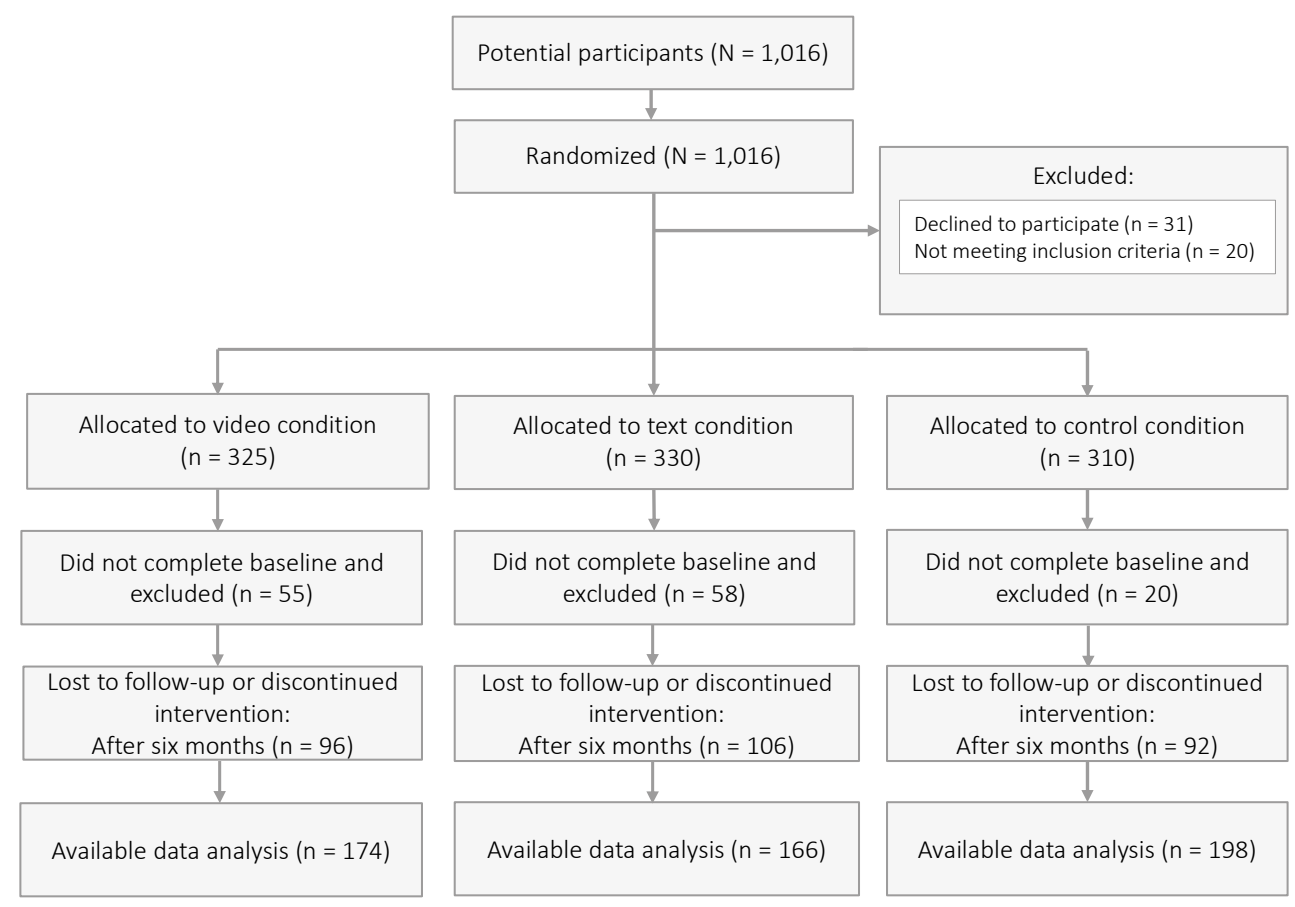

Figure 3 Flow diagram of Dutch adults $(N=1,016)$ randomized to three different conditions

Differences were also found in the level of addiction $(F(3,828)=6.48, p=.000)$. Those recruited from newspapers were less nicotine dependent than those recruited by the other three recruitment strategies. Moreover, there were differences in the samples with regard to the occurrence of COPD $\left(\chi^{2}=11.55, \mathrm{df}=3, p=.009\right)$. The GP sample consisted of more smokers who indicated to have COPD compared to recruitment via Internet. Finally, we found a significant difference between the samples regarding readiness to quit $\left(\chi^{2}=15.13, \mathrm{df}=3, p=\right.$ .002). Those recruited via 'other' strategies were less motivated to stop smoking compared with respondents recruited via GPs and the Internet. 


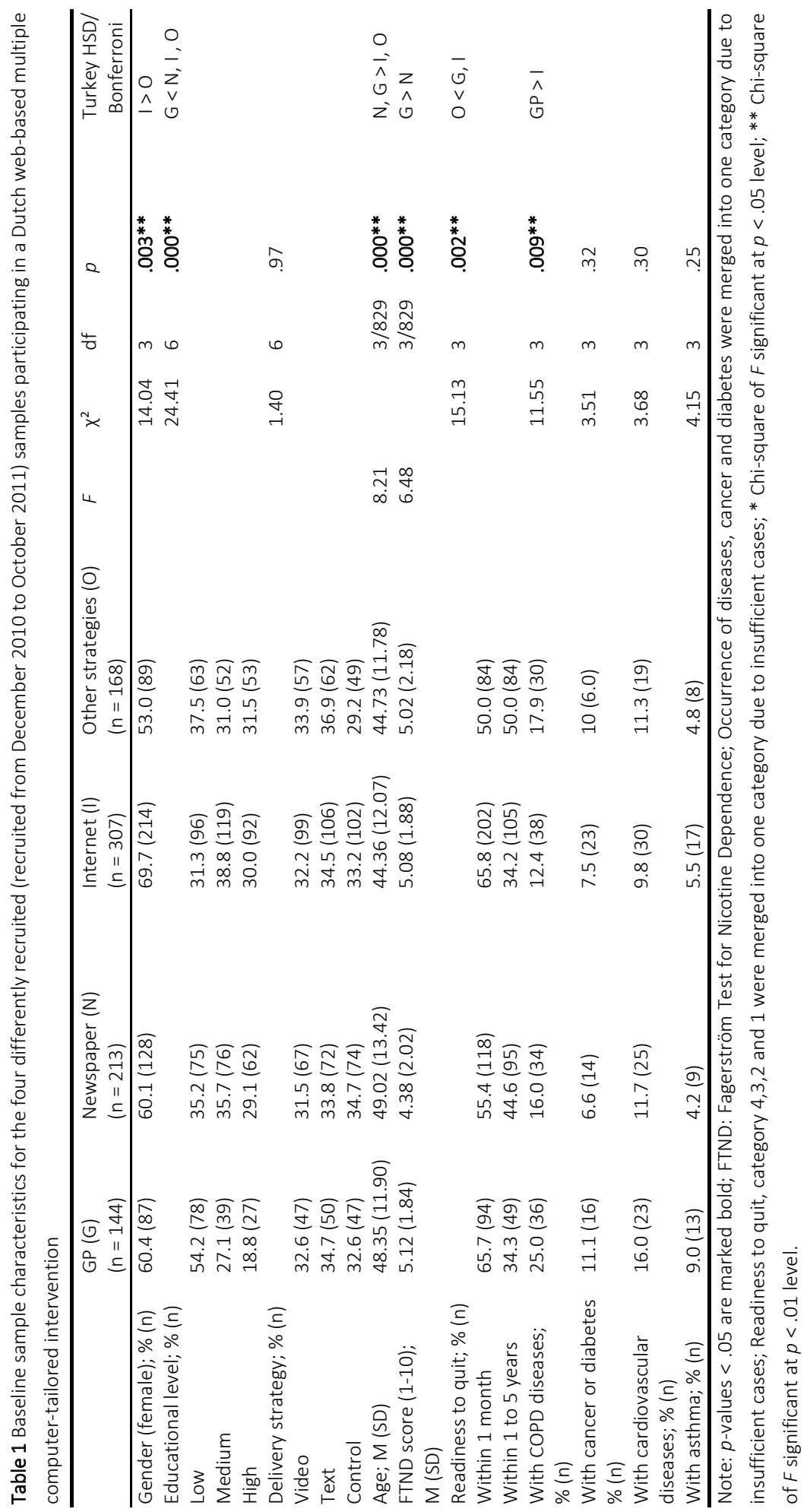




\section{Retention analysis}

Of the 144 eligible respondents who were recruited via GP practices, 99 (68.8\%) returned to the study after six months. Of the 213 eligible respondents who were recruited via newspaper advertisements, 151 (70.9\%) came back to fill out the follow-up. For the sample recruited via Internet advertisements ( $\mathrm{n}=307), 200$ (65.1\%) came back to fill out the follow-up questionnaire, whereas 88 (52.4\%) of the 168 eligible respondents recruited via other recruitment strategies returned to fill out the follow-up questionnaire. Possible predictors of retention for respondents who returned to the follow-up are presented in Table 2.

No interaction effect was found between educational level and mode of recruitment regarding retention $\left(\chi^{2}=2.97, \mathrm{df}=6, p=.81\right)$. Furthermore, no significant influence on retention was found for any of the three experimental conditions $\left(\chi^{2}=1.86, \mathrm{df}=6, p=.93\right)$. However, retention was significantly predicted by older age $(O R=1.02, p=.001)$ and a significant interaction effect was found between mode of recruitment and readiness to quit smoking $\left(\chi^{2}=6.42, d f=3, p=.09\right)$. Subsequent subgroup analysis revealed that for respondents who were less motivated to quit, those who were recruited by 'other' strategies were significantly less likely to return to the study compared with respondents recruited by GPS.

To test all possible combinations of each parameter pair, the same subgroup analysis was run again but newspaper, Internet and 'other recruitment' strategies were used as the reference group respectively (not presented in table). We found that respondents, within the group of less motivated respondents (one month to five years), and who were recruited via the Internet were significantly less likely to return to the study compared with respondents recruited via newspapers $(O R=0.91, p=.01)$. The same was true for 'other' strategies; respondents within the group of less motivated (one month to five years) and recruited from 'other' strategies were less likely to return to the study compared with respondents recruited via newspapers (OR $=0.34, p=.001$ ). Finally, we found that respondents within the group of highly motivated respondents (those wanting to quit 'within the next month') and recruited by 'other' strategies were less likely to return to the study compared with those recruited via the Internet $(\mathrm{OR}=0.53, p=.02)$.

\section{Differences in smoking behaviour: Quit attempts and seven-day point prevalence abstinence}

At follow-up, 72 (73.5\%) respondents recruited via GP practices indicated having made a serious attempt to quit smoking during the last six months, whereas 95 (65.1\%) respondents in the newspaper sample, 150 (77.7\%) respondents within the Internet advertisement sample and $55(67.1 \%)$ respondents within the 'other' strategies sample reported so. The differences in quit attempts between the recruitment groups were borderline significant $\left(\chi^{2}=7.62, \mathrm{df}=3\right.$, $p=.05)$. Predictors of having made a quit attempt in the previous six months are presented in Table 3. 
Table 2 Predictors of retention in Dutch adults recruited from December 2010 to October 2011- final regression model and subgroup analysis based on readiness to quit smoking

\begin{tabular}{|c|c|c|c|c|c|}
\hline \multicolumn{6}{|l|}{ After 6 months follow up } \\
\hline Final regression model & $\chi^{2}$ & $\mathrm{df}$ & OR & $95 \% \mathrm{Cl}$ & $p$ \\
\hline Newspaper recruitment $^{a}$ & & & 1.13 & $0.51-2.48$ & .77 \\
\hline Internet recruitment a & & & 0.54 & $0.26-1.14$ & .11 \\
\hline Other recruitment ${ }^{a}$ & & & 0.38 & $0.18-0.82$ & .01 \\
\hline Gender b & & & 1.21 & $0.89-1.65$ & .23 \\
\hline Low educational level c & & & 0.86 & $0.59-1.26$ & .44 \\
\hline Medium educational level ${ }^{c}$ & & & 0.96 & $0.66-1.39$ & .82 \\
\hline Age & & & 1.02 & $1.01-1.04$ & .001 \\
\hline FTND score (addiction level) & & & 1.03 & $0.95-1.11$ & .45 \\
\hline Experimental group (text) ${ }^{d}$ & & & 0.88 & $0.62-1.26$ & .50 \\
\hline Experimental group (video) $d$ & & & 0.96 & $0.66-1.38$ & .81 \\
\hline COPD e & & & 1.04 & $0.69-1.57$ & .86 \\
\hline Readiness to quit (within next month) ${ }^{f}$ & & & 0.78 & $0.36-1.68$ & .52 \\
\hline Readiness to quit $*$ recruitment & 6.42 & 3 & & & .09 \\
\hline
\end{tabular}

Subgroup analyses

\begin{tabular}{|c|c|c|c|}
\hline Readiness to quit (within next month) $(n=498$ ) & OR & $95 \% \mathrm{Cl}$ & $p$ \\
\hline Newspaper recruitment $^{a}$ & 1.03 & $0.57-1.87$ & .92 \\
\hline Internet recruitment ${ }^{a}$ & 1.27 & $0.74-2.19$ & .36 \\
\hline Other recruitment ${ }^{a}$ & 0.67 & $0.36-1.25$ & .21 \\
\hline Gender ${ }^{b}$ & 1.45 & $0.96-2.19$ & .08 \\
\hline Low educational level ${ }^{c}$ & 0.96 & $0.59-1.56$ & .86 \\
\hline Medium educational level c & 1.06 & $0.66-1.71$ & .82 \\
\hline Age & 1.02 & $1.00-1.04$ & .02 \\
\hline FTND score (addiction level) & 1.01 & $0.91-1.12$ & .88 \\
\hline Experimental group (text) ${ }^{d}$ & 0.69 & $0.43-1.10$ & .12 \\
\hline Experimental group (video) d & 0.80 & $0.49-1.31$ & .38 \\
\hline COPD e & 0.86 & $0.49-1.52$ & .61 \\
\hline Readiness to quit ( 1 month to 5 years) $(n=333)$ & OR & $95 \% \mathrm{Cl}$ & $p$ \\
\hline Newspaper recruitment $^{\mathrm{a}}$ & 1.10 & $0.49-2.46$ & .81 \\
\hline Internet recruitment ${ }^{a}$ & 0.51 & $0.24-1.08$ & .08 \\
\hline Other recruitment ${ }^{a}$ & 0.37 & $0.17-0.81$ & .01 \\
\hline Gender b & 0.98 & $0.60-1.58$ & .92 \\
\hline Low educational level c & 0.74 & $0.40-1.39$ & .36 \\
\hline Medium educational level c & 0.81 & $0.43-1.50$ & .50 \\
\hline Age & 1.02 & $1.00-1.04$ & .03 \\
\hline FTND score (addiction level) & 1.06 & $0.94-1.19$ & .35 \\
\hline Experimental group (text) ${ }^{d}$ & 1.26 & $0.72-2.21$ & .42 \\
\hline Experimental group (video) ${ }^{d}$ & 1.16 & $0.66-2.03$ & .61 \\
\hline COPD e & 1.30 & $0.70-2.42$ & .41 \\
\hline
\end{tabular}

Note: $p$-values < .05 are marked bold; ${ }^{a}$ General practitioner is the reference group (score $=0$ ); ${ }^{b}$ Female is the reference group (score $=0$ ); ${ }^{c}$ High educational level is the reference group (score $=0$ ); ${ }^{d}$ Control group is the reference group $\left(\right.$ score $=0$ ); ${ }^{\circ}$ Suffering from the disease is the reference group (score $=0) ;{ }^{f}$ Readiness to quit after 1 month to 5 years of later is the reference group (score $=0$ ).

No interaction effect was found between educational level and mode of recruitment regarding quit attempts $\left(\chi^{2}=3.66, d f=6, p=.72\right)$. Again, no differences in quit attempts were found between the three experimental condition $\left(\chi^{2}=1.77, \mathrm{df}=6, p=.94\right)$. 
Subgroup analysis among respondents with lower (FTND score < 5) and higher (FTND score > 5) levels of addiction showed that highly addicted respondents, recruited via newspapers, were significantly less likely to have made a quit attempt compared with the GP sample.

To test all possible combinations of each parameter pair, the same subgroup analyses were run again but the newspaper, Internet and 'other' recruitment strategies, respectively, were used as the reference group (not presented in Table). In the group of highly addicted smokers, respondents who were recruited via the Internet were more likely to make a quit attempt compared with those recruited via newspapers (OR $=2.86, p=.02$ ). In Table 4, descriptive data of the differently recruited samples are shown, stratified by level of addiction. The GP sample included especially higher addicted older women. When drop-outs were regarded as smokers, interaction effects between addiction level and mode of recruitment were no longer detected $\left(\chi^{2}=4.65, \mathrm{df}=3, p=.12\right)$.

At follow-up, 36 (36.4\%) respondents recruited via GP practices reported being abstinent during the last seven days. For the sample recruited via newspapers, 40 (26.5\%) respondents reported seven-day point prevalence abstinence, 64 (32.0\%) respondents recruited via Internet advertisements and 20 (22.7\%) respondents recruited via other recruitment strategies reported so. Though abstinence rates did not differ significantly between the different recruitment groups $\left(\chi^{2}=5.40, d f=3, p=.15\right)$. Logistic regression analysis regarding seven-day point prevalence abstinence did not reveal an interaction between educational level and mode of recruitment $\left(\chi^{2}=1.79, \mathrm{df}=6, p=.94\right)$ nor an interaction between addiction level and mode of recruitment $\left(\chi^{2}=2.60, d f=3, p=.46\right)$. Furthermore, no differences were found with regard to seven-day point prevalence abstinence between the three experimental conditions $\left(\chi^{2}=1.89, \mathrm{df}=6, p=.93\right)$. However, there was a significant interaction effect between mode of recruitment and gender $\left(\chi^{2}=8.08, \mathrm{df}=3, p=.04\right)$. Subsequent subgroup analysis among gender revealed that women, recruited via newspapers were significantly less likely to report being abstinent compared with women, recruited via GPs (see Table 5). Next, women in the video condition were more likely to report being abstinent compared to women in the control condition.

To test all possible combinations of each parameter pair, the same subgroup analysis was run but newspaper, Internet and 'other' strategies were used respectively as the reference group (not presented in Table). It was shown that men recruited via 'other' strategies were less likely to quit compared to men recruited via newspapers (OR $=0.32, p=.03$ ) and compared with men recruited via the Internet $(O R=0.34, p=.04)$. When drop-outs were regarded as smokers, no significant differences were found between gender $\left(\chi^{2}=5.12\right.$, $\left.d f=3, p=.16\right)$. It was shown that men recruited via 'other' strategies were less likely to quit compared to men recruited via newspapers $(\mathrm{OR}=0.32, p=.03)$ and compared with men recruited via the Internet $(\mathrm{OR}=0.34, p=.04)$. When drop-outs were regarded as smokers, no significant differences were found between gender $\left(\chi^{2}=5.12, \mathrm{df}=3, p=.16\right)$. 


\section{Costs per smoker reached}

On average, €4,643.81 was spent on the recruitment via GPs for recruitment materials (e.g. addresses of GPs and flyers). Time that the research team as well as the GPs spent on the inclusion and preparation (e.g. GPs had to get informed about the intervention) were not included in the calculation. Furthermore, $€ 1,792.42$ was spent on the advertisements in the newspapers, $€ 750.00$ was spent on the recruitment via Internet advertisements and $€ 378.03$ was spent on the recruitment via companies. Investments in terms of the research team were again not taken into account. One hundred and forty-four eligible smokers were recruited through GPs. The costs per eligible respondent to initiate the intervention were thus $€ 32.24$ (€442.81/144). Two hundred and thirteen eligible smokers were recruited via newspaper advertisements, resulting in $€ 8.42(€ 1,792.42 / 213)$ spent per recruited smoker. Three hundred and seven smokers were recruited via the Internet resulting in $€ 2.44$ (€750.00/307) spent per recruited smoker whereas 168 eligible smokers were recruited by 'other' strategies resulting in $€ 2.25$ (€378.03/168) spent per recruited smoker. For every respondent having made a serious quit attempt approximately, €64.48 (GPs), €18.87 (newspaper), €5 (Internet) and €6.87 ('other' strategies) were spent. For every respondent who reported being abstinent during the seven-day point prevalence abstinence prior to the measurement $€ 128.96$ (GPs), $€ 44.81$ (newspaper), €11.72 (Internet) and €18.90 ('other' strategies) were spent on recruitment. 


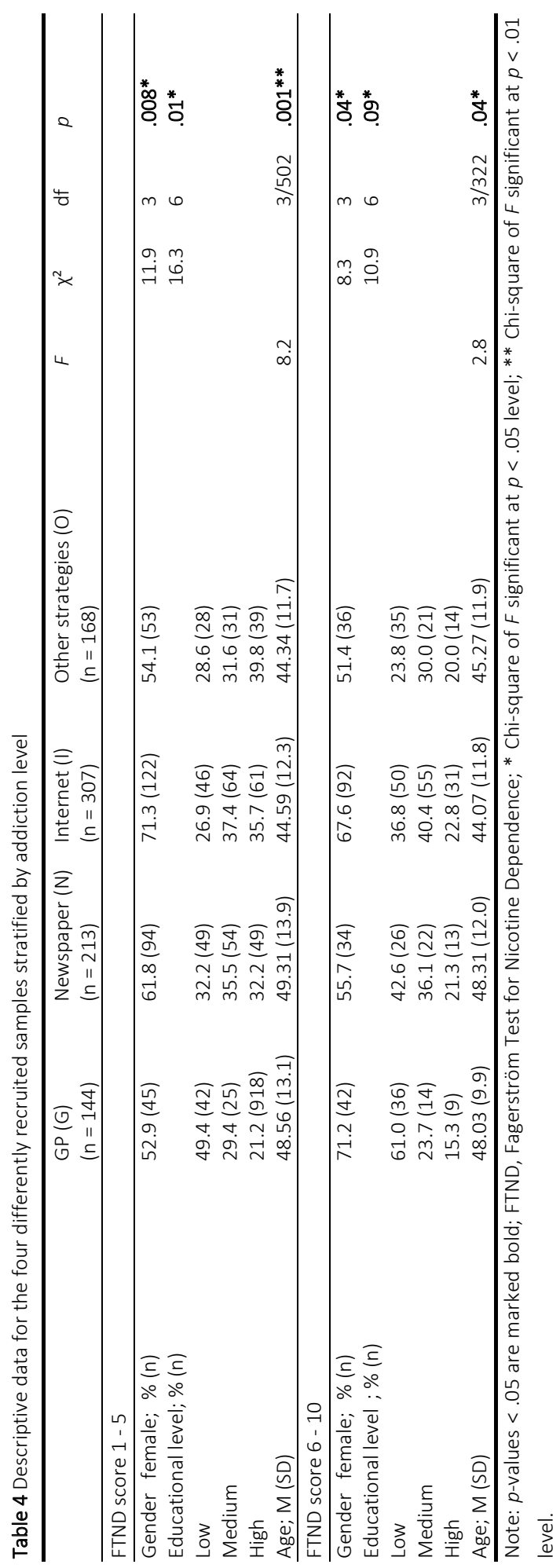


Table 5 Predictors of seven-day point prevalence abstinence in Dutch adults recruited from December 2010 to October 2011final regression model and subgroup analysis based on gender

\begin{tabular}{|c|c|c|c|c|c|}
\hline \\
\hline$\frac{\text { After } 6 \text { months follow up }}{\text { Final regression model }}$ & $\chi^{2}$ & df & OR & $95 \% \mathrm{Cl}$ & $p$ \\
\hline Newspaper recruitment ${ }^{a}$ & & & 0.35 & $0.17-0.73$ & .005 \\
\hline Internet recruitment a & & & 0.59 & $0.30-1.13$ & .11 \\
\hline Other recruitment ${ }^{a}$ & & & 0.60 & $0.26-1.40$ & .23 \\
\hline Gender b & & & 0.55 & $0.23-1.30$ & .17 \\
\hline Low educational level $\mathrm{c}$ & & & 0.97 & $0.60-1.58$ & .91 \\
\hline Medium educational level ${ }^{c}$ & & & 1.12 & $0.69-1.82$ & .64 \\
\hline Age & & & 1.01 & $0.99-1.03$ & .31 \\
\hline FTND score (addiction level) & & & 0.98 & $0.89-1.08$ & .74 \\
\hline Experimental group (text) ${ }^{d}$ & & & 1.03 & $0.64-1.65$ & .92 \\
\hline Experimental group (video) ${ }^{d}$ & & & 1.31 & $0.82-2.08$ & .27 \\
\hline COPD e & & & 1.12 & $0.66-1.89$ & .68 \\
\hline Readiness to quit (within next month) ${ }^{f}$ & & & 1.29 & $0.86-1.92$ & .22 \\
\hline Recruitment * Gender & 8.078 & 3 & & & .04 \\
\hline
\end{tabular}

Subgroup analyses

\begin{tabular}{|c|c|c|c|}
\hline Men $(n=212)$ & OR & $95 \% \mathrm{Cl}$ & $p$ \\
\hline Newspaper recruitment ${ }^{a}$ & 1.50 & $0.60-3.72$ & .39 \\
\hline Internet recruitment ${ }^{a}$ & 1.39 & $0.58-3.36$ & .46 \\
\hline Other recruitment ${ }^{a}$ & 0.48 & $0.16-1.43$ & .19 \\
\hline Low educational level ${ }^{c}$ & 1.14 & $0.51-2.51$ & .75 \\
\hline Medium educational level c & 1.10 & $0.50-2.40$ & .82 \\
\hline Age & 1.00 & $0.98-1.03$ & .84 \\
\hline FTND score (addiction level) & 1.01 & $8.75-1.18$ & .85 \\
\hline Experimental group (text) $d$ & 0.94 & $0.46-1.93$ & .86 \\
\hline Experimental group (video) ${ }^{d}$ & 0.68 & $0.32-1.47$ & .33 \\
\hline COPD e & 1.04 & $0.42-2.55$ & .94 \\
\hline Readiness to quit (within next month) ${ }^{f}$ & 1.15 & $0.61-2.19$ & .67 \\
\hline Women $(n=325)$ & OR & $95 \% \mathrm{Cl}$ & $p$ \\
\hline Newspaper recruitment $^{\text {a }}$ & 0.34 & $0.16-0.72$ & .005 \\
\hline Internet recruitment ${ }^{a}$ & 0.59 & $0.30-1.17$ & .13 \\
\hline Other recruitment ${ }^{a}$ & 0.58 & $0.24-1.37$ & .21 \\
\hline Low educational level c & 0.89 & $0.47-1.69$ & .73 \\
\hline Medium educational level c & 1.26 & $0.67-2.39$ & .48 \\
\hline Age & 1.02 & $0.99-1.04$ & .18 \\
\hline FTND score (addiction level) & 0.94 & $0.82-1.07$ & .33 \\
\hline Experimental group (text) ${ }^{d}$ & 1.23 & $0.64-2.34$ & .54 \\
\hline Experimental group (video) ${ }^{d}$ & 2.13 & $1.14-4.01$ & .02 \\
\hline COPD e & 1.21 & $0.62-2.37$ & .57 \\
\hline Readiness to quit (within next month) ${ }^{f}$ & 1.34 & $0.80-2.27$ & .27 \\
\hline
\end{tabular}

Note: $p$-values < .05 are marked bold; a General practitioner is the reference group (score $=0$ ); ${ }^{b}$ Female is the reference group $($ score $=0) ;{ }^{c}$ High educational level is the reference group (score $\left.=0\right) ;{ }^{d}$ Control group is the reference group $(\operatorname{score}=0)$; ${ }^{e}$ Suffering from the disease is the reference group (score $=0) ;{ }^{f}$ Readiness to quit after 1 month to 5 years of later is the reference group (score $=0$ ).

\section{Discussion}

\section{Main findings}

The aim of this study was to investigate whether different recruitment strategies resulted in different samples of smokers participating in the computer-tailored smoking cessation intervention with emphasis on differences between lower and higher educated smokers. 
First, our results revealed that the GP recruitment attracted a larger proportion of lower educated smokers and more smokers suffering from COPD compared with the other recruitment strategies. Second, smokers recruited via newspapers were shown to be less nicotine dependent compared with smokers recruited via other methods. In addition, the results showed that older respondents were more likely to return to the study at follow-up. The effect of recruitment strategy also depended on the readiness of the respondent to quit smoking. Retention rates among people with a lower readiness to quit were higher among those recruited by GPs compared with those recruited via 'other strategies'. Third, our results furthermore suggested that highly addicted respondents recruited via newspapers were less likely to make a quit attempt compared with highly addicted respondents recruited via GPs or the Internet. With regard to seven-day point prevalence abstinence, women recruited via newspapers were less likely to be abstinent compared with women recruited via GPs. None of the variables were predictive for men. No significant differences were found between the three conditions regarding retention, quit attempts and seven-day point prevalence abstinence. Fourth, our study findings clearly demonstrate that costs when using GPs, recruitment and quitting behaviours were much higher than for any other modality. No significant differences were found between lower and higher educated smokers regarding retention and smoking behaviour.

Supporting findings of previous national and international studies, the recruitment via GPs resulted in a relatively larger population of lower educated smokers and smokers with smoking-related diseases who participated and made more quit attempts compared with recruitment via other strategies (Belisario et al., 2012, Hoving et al., 2007, Smit et al., 2011). It is conceivable that lower educated smokers with smoking-related diseases visit a GP more often than other educational groups, thus resulting in the fact that recruitment via GPs reaches relatively more lower educated smokers. However, when absolute numbers are used, the results show that recruitment via the other three strategies was more successful in reaching more smokers of a lower educated level. Hence, from a public health impact point of view (Graham et al., 2008), this would favour approaches other than recruitment via GPs. If the target group of smokers is not limited to lower educated smokers, recruitment via the Internet and newspaper advertisements may possibly be a better and cheaper strategy to recruit a high number of smokers, something also found in previous research (Smit et al., 2011). Furthermore, our results showed that the more traditional recruitment approaches (e.g. the recruitment via newspapers) seemed to attract a lower percentage of nicotine dependent smokers compared with recruitment via the Internet (Graham et al., 2008). A possible explanation could be that the less addicted smokers, which could be also categorized into the cold-contacted group (Balmford et al., 2013), might have become by chance attentive of the newspaper advertisement compared with higher addicted respondents who might be more 
proactive in seeking cessation guidance (e.g. asking GPs for advice or searching on the Internet).

In line with previous research (Smit et al., 2011), we furthermore found that retention rates among people with a lower readiness to quit were higher among those recruited by GPS compared with recruitment via 'other strategies'. Next, our results revealed that highly addicted smokers recruited by the GPs and the Internet, were more likely to make a quit attempt compared with highly addicted people recruited by newspapers. A possible reason could be that respondents recruited via the GPs received more smoking cessation support, were more motivated to quit and more interested in quitting compared with the sample recruited via newspapers (Webb et al., 2010). Again perhaps, respondents recruited via the Internet or GPs might have actively searched for quit programs and were more involved in quitting compared with the more 'cold-contacted' groups, such as the newspaper sample (Balmford et al., 2013). Furthermore, the three conditions to which respondents were assigned showed no impact on retention and smoking-related outcomes. Although this was not our main research question in this study, we would have expected respondents in the experimental conditions to be more likely to return to the study and quit smoking compared with respondents in the control condition. In line with past research, the costs per recruited smokers, per quit attempt made and per abstinent respondent were the highest among the GP sample compared with the other strategies (Smit et al., 2011). We were not able to replicate our findings when drop-outs were regarded as smokers. However, since we had no information about why respondents did not return to the follow-up measurements, treating all missing cases as treatment failures may be too conservative.

In sum, the recruitment via GPs might be an effective, but also expensive, way of recruiting lower educated smokers and respondents with more smoking-related diseases, whereas recruitment via mass media channels resulted in a larger absolute number of smokers, and also lower educated smokers. This study therefore suggests that future trials might use the support of mass media channels to recruit respondents since many more smokers were recruited with this strategy.

\section{Limitations}

Several limitations should be noted. First, it was only known how many smokers enrolled in the intervention but not known how many smokers were actually invited per strategy (e.g. by GPs) and how many respondents visited the website. Therefore, it was not possible to calculate the intervention's public health impact (Abrams et al., 1996), which might be valuable to include in future studies. Second, GP recruitment resulted in relatively few smokers. Since the procedure might have been unclear to them, future research should include face-to-face meetings, to avoid indistinctness. In addition, the changes in financial compensation for smoking cessation pharmacotherapy in the Netherlands (2011) might have also influenced the 
enrolment of smokers. Third, the evaluation of the recruitment strategy mainly relied on one question, asking respondents how they learned about the intervention. Because several recruitment strategies were used simultaneously, and respondents may have become aware of the intervention via different channels, this could have caused errors in recall. Fourth, given the fact that socioeconomic status (SES) is a multidimensional construct, it might be that educational level alone was not sufficient to accurately assess SES and therefore limited our results. However, past research has already indicated educational level to be a good indicator of SES (Hiscock et al., 2011, Schaap et al., 2009). Fifth, due to financial reasons, we were not able to conduct biochemical validation to confirm self-reported smoking status. Quit rates may therefore have been overestimated. It is however unlikely that misreports of smoking status varied between the recruitment channels and is therefore not expected to bias the results on our main research questions (Velicer et al., 1992). Sixth, referring respondents to a web-based Internet intervention might have influenced the sample being studied. However, since the Internet in the Netherlands is highly accessible to smokers with different educational levels, we do not think that this could have biased our results. In addition, we measured retention after six months, however, it might be also valuable to investigate how respondents adhere to the computer-tailored program during the intervention period itself (e.g. which sessions they followed, whether there are differences in adherence between routing 1 and 2) and to assess long-term effects. Finally, smokers were recruited to participate for a smoking cessation trial; however the results might be different when recruiting smokers for interventions.

\section{Conclusion}

The purpose of this study was to obtain a better understanding of which recruitment strategies are most effective when inviting smokers for a smoking cessation intervention. We can conclude that mode of recruitment influenced the type of smokers, participating in the computer-tailored smoking cessation intervention. The results of our study showed that GPs recruited a higher percentage of lower educated smokers who participated and made a quit attempt compared with the other strategies. However, when using absolute numbers, our results showed that recruitment via other strategies resulted in a larger absolute number of (lower educated) smokers who participated and made a quit attempt. Finally, our findings clearly show that the costs when using GP recruitment were much higher than for any other recruitment strategy. 


\section{CHAPTER 4}

Influence of delivery strategy on message processing mechanisms and future adherence to a Dutch computer-tailored smoking cessation intervention

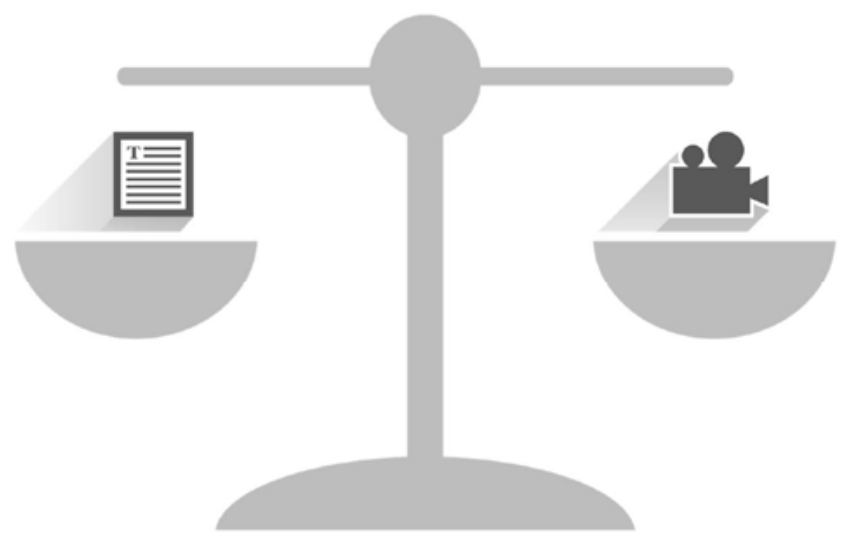

Published as:

Stanczyk, N. E., Crutzen, R., Bolman, C., Muris, J. W. M. \& de Vries, H. (2013). Influence of delivery strategy on message processing mechanisms and future adherence to a Dutch computer-tailored smoking cessation intervention. Journal of Medical Internet Research, 15, e28. 


\section{Abstract}

Background: Smoking tobacco is one of the most preventable causes of illness and death. Webbased tailored smoking cessation interventions have shown to be effective. Although these interventions have the potential to reach a large number of smokers, they often face high attrition rates, especially among lower educated smokers. A possible reason for the high attrition rates in the latter group is that computer-tailored smoking cessation interventions may not be attractive enough as they are mainly text-based. Video-based messages might be more effective in attracting attention and stimulating comprehension in people with a lower educational level and could therefore reduce attrition rates.

Objective: The objective of the present study was to investigate whether differences exist in message-processing mechanisms (attention, comprehension, self-reference, appreciation, processing) and future adherence (intention to visit/use the website again, recommend the website to others) according to delivery strategy (video- or text-based messages) and educational level toward a Dutch computer-tailored smoking cessation program.

Methods: Smokers who were motivated to quit within the following six months and who were aged over 16 were included in the program. Respondents were randomly assigned to one of two conditions (video/text computer tailoring). The sample was stratified into two categories: low and high educated respondents. In total, 139 respondents completed the first session of the web-based tailored intervention and were subsequently asked to fill out a questionnaire assessing message-processing mechanisms and future adherence. ANOVAs and regression analyses were conducted to investigate the differences in message-processing mechanisms and future adherence with regard to delivery strategy and education.

Results: No interaction effects were found between delivery strategy (video vs. text) and educational level on message-processing mechanisms and future adherence. Delivery strategy had no effect on future adherence and processing mechanisms. However, in both groups results indicated that lower educated respondents showed higher attention $(F(1,138)=3.97$, $p=.05)$ and processing levels $(F(1,138)=4.58, p=.04)$. Results revealed also that lower educated respondents were more inclined to visit the computer-tailored intervention website again $(F(1,138)=4.43, p=.04)$.

Conclusions: Computer-tailored programs have the potential to positively influence lower educated groups as they might be more involved in the computer-tailored intervention than higher educated smokers. Longitudinal studies with a larger sample are needed to gain more insight into the role of delivery strategy in tailored information and to investigate whether the intention to visit the intervention website again results in the ultimate goal of behaviour change.

Keywords: computer tailoring, smoking cessation, message-processing mechanisms, future adherence 


\section{Introduction}

During the last decade, an increasing number of people used the Internet to obtain healthrelated information. In the field of health promotion, the Internet has become an important medium for the delivery of behavioural change interventions (Leung, 2008, Oenema et al., 2008). Health professionals have started to deliver several lifestyle behaviour interventions through the web, including interventions aimed at smoking cessation (Te Poel et al., 2009), nutrition behaviour (Oenema et al., 2001), and physical activity (Peels et al., 2012, Steele et al., 2007).

A successful interactive strategy consists of computer-tailored interventions (De Vries and Brug, 1999) through which individuals receive personalised information and feedback on health behaviour and motives for this behaviour. Tailored health messages are based on a person's answers to a questionnaire on individual characteristics related to health behaviour. Current research has shown the superiority of tailored materials over existing standard materials (Civliak et al., 2010, Dijkstra, 2005). In the field of smoking cessation, recent studies have revealed computer-tailored smoking cessation interventions to be more effective than non-tailored interventions (Civliak et al., 2010, Myung et al., 2009). Yet, as with many e-health interventions, smoking cessation interventions delivered via the Internet have high attrition rates, especially among people with lower education (Bennett and Glasgow, 2009, Eysenbach, 2005). Yet, less educated people are often those who smoke more cigarettes (Nagelhout et al., 2012) and show higher nicotine addiction rates, less quit-attempts, and more relapses compared to people with a higher level of education (Brouwer et al., 2010, Hiscock et al., 2011). Past research in the Netherlands has shown that smoking prevalence was significantly higher among less educated people (29\%) compared to people with a higher level of education (20\%) (Nagelhout et al., 2012). Smokers of lower socioeconomic status (LSES) are therefore a highly relevant target group for using these computer-tailored programs. Hence, it is important to investigate how attrition rates among lower educated groups can be reduced and how computer-tailored interventions can be optimised to attract different groups of different education levels.

A possible reason for the high attrition rates in computer-tailored interventions is that they heavily rely on text-based messages. Research suggested that video-based messages might be more effective in attracting attention and stimulating comprehension in people with a lower educational level (Houts et al., 2006, Nielsen and Sheppard, 1988). Furthermore, videobased messages have been shown to require less mental effort and may help the person focus on the core elements of the message (Salomon, 1984, Salomon and Gardner, 1986). It is therefore conceivable that video-based messages may be better for reaching lower educated groups and realizing behaviour change. Additionally, higher educated groups may benefit more 
from in-depth processing and accordingly may be stimulated more by text-based messages (Soetens et al., 2014).

Recent research examining computer tailoring identified different underlying messageprocessing mechanisms that play an important role in enhancing health communication (Hawkins et al., 2008). In tailored communication, five important message-processing mechanisms have been identified: attention, comprehension, processing, self-reference, and appreciation. Attention refers to the ability to attend the receiving message. Due to the fact that paying less attention to a message lowers the overall effect, one purpose of tailored material is to increase the attention to the message. A recent study has shown stronger attention processes in people reading tailored material (Kessels et al., 2011). Comprehension refers to the ability to understand the content of the message. Past studies have shown that the better the message was matched to personal attributes and preferences, the more the message was understood and remembered (Campbell et al., 1994, Skinner et al., 1994). A psychological theory that has been used to explain the effects of tailoring concerns the Elaboration Likelihood Model (ELM). This theory states there are two processing routes, the peripheral and the central. Related to this theory, personally relevant messages are processed by the "central route" and therefore take more effort to process (Petty and Cacioppo, 1986). The idea of tailoring is to increase the perceived relevance of the message in order to elicit a careful consideration of the message, which leads to a deeper impact of the received content. Indeed, a study about weight loss information indicated respondents engaged in deeper processing of tailored information when compared to non-tailored information (Kreuter et al., 1999b). Besides effortful processing, a further aim of tailoring is to stimulate self-referential thinking. Self-referential thinking refers to the ability to refer the given information to one's own situation. For example, tailored weight loss materials have been shown to encourage respondents to link the information to their own situation (Kreuter et al., 1999b). Furthermore, it is also theorized that tailoring influences the appraisal of a message. Tailored materials may enhance the feeling of being well understood and would therefore lead to more appreciation compared to non-tailored information. To sum up, tailoring is used to increase the relevance of a health message by stimulating attention, comprehension, and the overall depth of message processing. The effects of tailoring on message-processing mechanisms might be further increased by the use of a suitable delivery strategy. However, as already indicated, these possible effects might be different for people with a lower or higher educational level.

To our knowledge, no previous studies have empirically examined the effects of delivery strategies (video vs. text) and their impact on message-processing mechanisms among different educational groups. The first aim of this paper was to explore whether there exist differences in message-processing mechanisms according to delivery strategy (video- or textbased messages) and educational level. Past research has shown that information processing in lower educated groups was less profound and more influenced by visual than textual 
information (Cacioppo et al., 1996). Based on this, it was expected that tailoring would result in deeper information processing in lower educated smokers who received the video-based messages. In contrast, it was expected that the effects of tailoring would increase for higher educated smokers who received the text-based messages.

Additionally, e-health research has acknowledged the importance of user experience of the intervention website. User experience refers to what a person thinks and feels during and after being exposed to a website (Crutzen et al., 2009b). Past research demonstrated the importance of user experiences (e.g. trustworthiness, enjoyment) with regard to re-visiting the website (Cyr et al., 2009). A positive user experience is related to an increased website use (Crutzen et al., 2009b), resulting in future adherence (Crutzen et al., 2011). Future adherence can comprise two components: (1) the intention to revisit an Internet-delivered intervention, and (2) recommending an Internet-delivered intervention to others (Crutzen et al., 2011). Internet delivered interventions and especially computer-tailored interventions often consist of several modules/feedback sessions. Since several sessions are often necessary to achieve behaviour change in the long run (Strecher et al., 2008b, Verheijden et al., 2007), it is important to know whether people would like to revisit the intervention website. People with a high intention to revisit the website intervention might be likely to follow further important parts/sessions of the intervention and could therefore benefit more from the intervention than people leaving the program after their first visit. Next, it is important to know whether people would recommend the Internet-delivered intervention to others since previous studies demonstrated that the "word of mouth" strategy is effective in order to increase the use of ehealth interventions (Crutzen et al., 2009c). In order for web-based interventions to have an impact on public health, it is important that the intervention is also disseminated by the target population (Glasgow et al., 1999). Now that computer-tailored interventions have become an increasingly popular strategy in the field of smoking cessation interventions, it is important to examine whether future adherence of computer-tailored intervention websites is influenced by delivery strategy (video vs. text) and whether these effects vary among different educational groups.

The second aim of this study was to investigate whether there exist differences in future adherence according to delivery strategy (video- or text-based messages) and educational level. It was expected that future adherence would increase in lower educated smokers who received the video-based messages; whereas we expected the same effects for higher educated smokers who received the text-based messages.

In summary, the current study was designed to assess whether a different delivery strategy (video vs. text) interacted with educational level on message-processing mechanisms and future adherence of a Dutch computer-tailored smoking cessation program. 


\section{Methods}

\section{Background}

This study aims to provide an in-depth exploration of the effects of video and text computer tailoring on message-processing mechanisms, which are also tested within the currently tested randomized controlled trial (RCT) (Stanczyk et al., 2011). Hence, the sample and the study we describe in this paper are different from the currently tested RCT. The described study uses only one session of the intervention tested within the RCT to test a different hypothesis, (i.e. differences between video/text on outcome measures regarding message-processing mechanisms and future adherence, which are not directly related to health, and not the hypothesis stated in the RCT protocol concerning the effect on smoking cessation) and was therefore not registered as a trial.

\section{Sample}

Respondents for the study were recruited in May 2011 through the Dutch Internet research agency Flycatcher (Flycatcher Internet Research, 2012). From this panel, a sample of 11,583 potential respondents was approached to fill in a short web-based questionnaire about their smoking behaviour, their motivation to quit, and their educational level. The main purpose of this pre-analysis was to include respondents who smoked only at the time of the study inclusion, who were aged 16 years or older, who were motivated to quit, and who could be categorized as lower or higher educated respondents. They were first asked to indicate whether they smoked. Respondents who indicated they smoke were next asked to indicate how often they smoked ranging from daily to once a month or less. Motivation to quit smoking was measured by one item assessing whether the respondent intended to quit smoking in the future on an eight-item scale ranging from 'definitely not' (1) to 'definitely yes' (8). All respondents who at least indicated that they were most likely (6) to quit in the near future were categorized as motivated. Educational level was divided into low (primary, basic vocational, lower general school), intermediate (higher general secondary education, preparatory academic education, medium vocational school) and high (higher vocational school or university level). Only respondents with a low or high educational level (categorized to the standards of Statistics Netherlands) were invited to take part in the study (CBS, 2012). In total, a random sample of 300 panel members who met these inclusion criteria was invited to participate in the study. From these, 240 clicked on the link of the intervention website and agreed to participate in the study (response rate: 80\%); 36 respondents did not fill out the questionnaire resulting in a sample of 204 respondents who finished the study (retention rate: $85 \%)$. From these 204 , only smokers who stayed more than five minutes on the intervention website ( $N=139$ ) were included in the main analysis. We used this inclusion criterion since a minimum of five minutes is needed to process the information in both conditions. In order test 
our hypotheses on message-processing mechanisms and future adherence correctly, we found it necessary to include only smokers who thoroughly completed the program. This resulted in a sample of 139 respondents (see Figure 1). As an additional strategy, we also conducted an intention-to-treat analysis of the data from all respondents, also including those who stayed less than five minutes on the website.

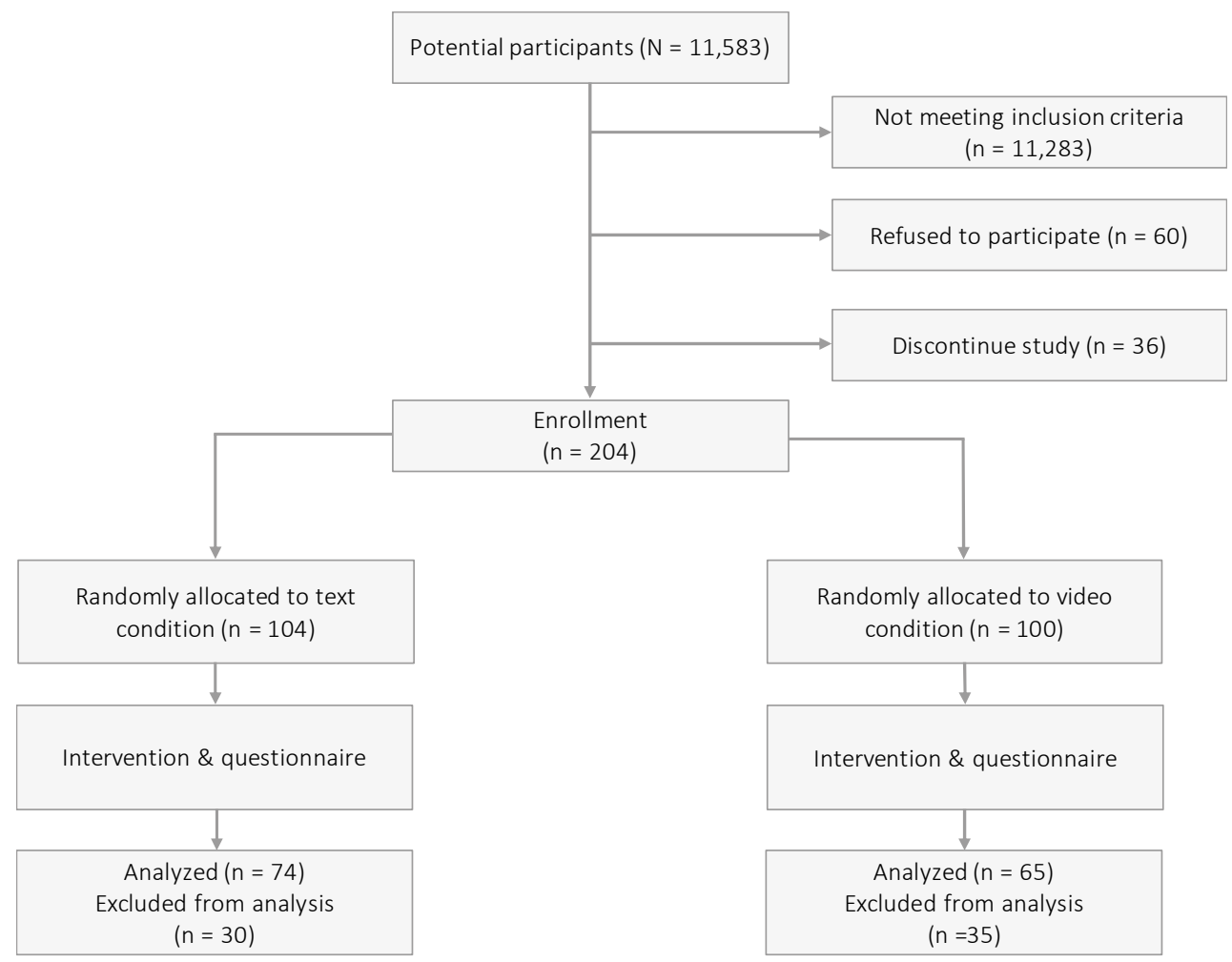

Figure 1 Flowchart of the study

\section{Procedure}

After signing up for participation and giving online informed consent, all included smokers were exposed to the website of a Dutch smoking cessation computer-tailored intervention (Steunbijstoppen.nl, 2012). Respondents were informed that they were able to discontinue their participation in the study at any time without any consequences. Smokers were randomly allocated to either the text condition or the video condition. Allocation to the two conditions was executed by the Tailor Builder computer software program (OSE, Sittard, the Netherlands). This software was developed for the execution of different web-based tailored interventions (OSE, 2011). Smokers had to follow only one session of the currently tested computer-tailored smoking cessation intervention. After completing this session, they were asked to fill out a 
web-based questionnaire assessing message-processing mechanisms and the intention to revisit the website and recommend it to others.

\section{Content}

Intervention

The computer-tailored smoking cessation intervention was based on the I-Change model (De Vries et al., 2003). Respondents in the text condition received computer-tailored text messages, whereas respondents in the video condition received computer-tailored video messages. In the text condition, respondents were presented text phrases without any further graphics or animations. For the video condition, the text-driven messages were translated into narrated video-driven messages that had a news-driven format with different adults delivering the tailored messages. We used simple videos without any other animation effects, like cartoons, hyperlinks, etc. In the video condition, the same tailored advice was used as in the text condition. The only difference between the two conditions was the strategy of delivery; the content of the advice was the same in both conditions (see Figure 2 and 3). Feedback messages were based on respondents answers to a questionnaire and tailored to their individual characteristics, such as their beliefs towards smoking, their intention to quit, and their overall smoking behaviour. The session smokers had to follow in this study was intended to increase respondents' motivation to quit smoking and to encourage smokers to quit smoking in the near future. First, smokers received three tailored feedback messages on their perceived advantages and disadvantages of quitting. Next, one piece of advice was offered with respect to respondents perceived social support. Last, one piece of tailored advice was provided on their perceived self-efficacy to quit smoking. A detailed description of the different intervention components that are assessed in the currently tested RCT are reported elsewhere (Stanczyk et al., 2011). 


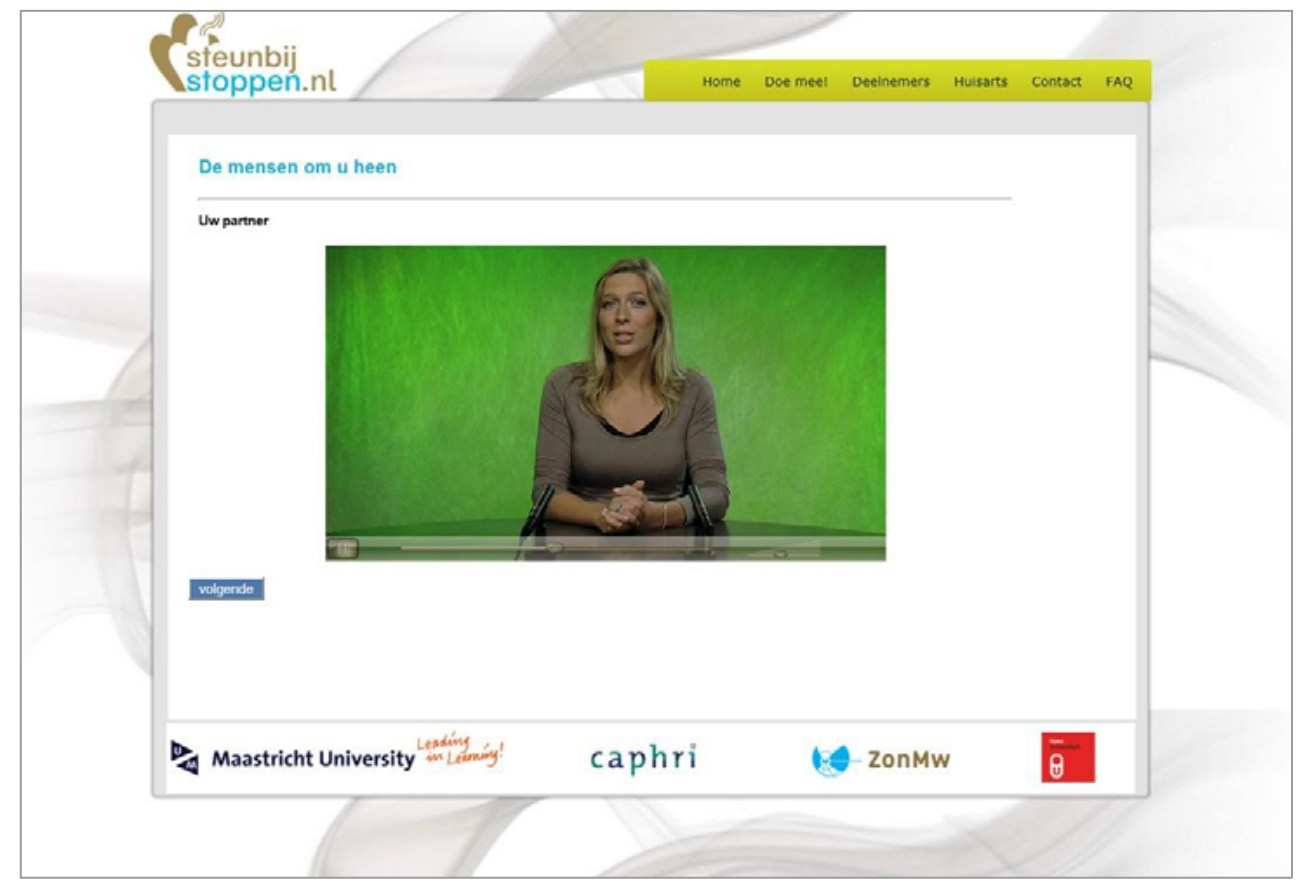

Figure 2 Screen shot of a personal advice of the video condition

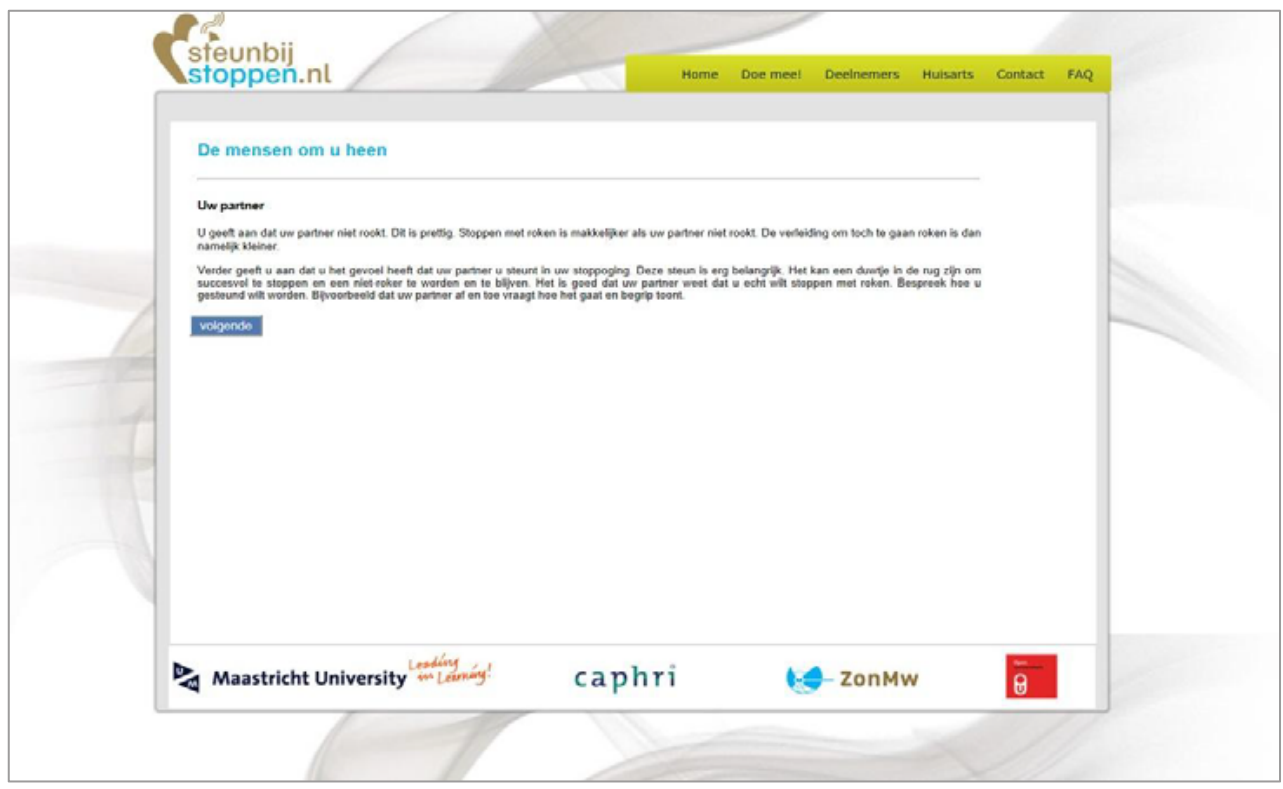

Figure 3 Screen shot of a personal advice of the text condition 


\section{Measures}

The following demographic variables were measured: age, gender ( $0=$ male; $1=$ female), and educational level $(0=$ low; 1 = high) (CBS, 2012). Furthermore, time spent on the intervention website was logged by the program system in minutes and seconds.

Addiction level was measured by six items using the Fagerström Test for Nicotine Dependence (FTND), asking respondents how many cigarettes they smoked per day, at which time points, and whether they had difficulties not smoking in smoke-free places. The answers were converted into an overall sum score ( 0 = not addicted; 10 = highly addicted) (Heatherton et al., 1991).

Readiness to quit smoking was measured by one item asking respondents whether they intended to quit smoking, resulting in six categories ( $6=$ yes, within the following month; 5 = yes, within one to three months; 4 =yes, within four to six months; 3 = yes, within one year; 2 = yes, within one and five years; 1 = yes, but not within the following five years) (De Vries et al., 2008, Dijkstra et al., 1996).

Cognitive processing (e.g. I like tasks where I do not have to think much, assessing to what extent people engage in effortful processing) was measured by six items on a five-point scale of the Heuristic Systematic Processing Questionnaire ranging from 5, "I totally agree" to 1 ,"I totally disagree" (Cronbach's alpha = .74) (Cacioppo and Petty, 1982).

Future adherence was measured by two concepts: intention and recommendation. Intention to revisit (e.g. It is likely that I will visit the website again in the future) was measured by three items on a seven-point scale, ranging from 7, "I totally agree" to 1,"I totally disagree" (Cronbach's alpha $=.91)$. Recommendation to others (e.g. It is likely that I will recommend this website to others) was measured by three items on a seven-point scale ranging from 7 "I totally agree" to 1, "I totally disagree" (Cronbach's alpha = .97) (Crutzen et al., 2011, Cyr et al., 2005).

User experiences (also known as user perceptions) (Crutzen et al., 2011) were measured by four concepts: trustworthiness, enjoyment, active trust, and design aesthetic. Trustworthiness (e.g. I trust the information presented on this website) was measured by three items on a seven-point scale ranging from 7, "I totally agree" to 1, "I totally disagree" (Cronbach's alpha = .87). Enjoyment (e.g. I found my visit to this website enjoyable) was measured by three items on a seven-point scale ranging from 7 , "I totally agree" to 1 , "I totally disagree" (Cronbach's alpha = .92). Active trust (e.g. I would act on the information presented on this website if needed) was measured by three items on a seven-point scale ranging from 7, "I totally agree" to 1 , "I totally disagree" (Cronbach's alpha = .91). Design aesthetic (e.g. The whole design of the website/program is attractive) was measured by three items on a sevenpoint scale ranging from 7, "I totally agree" to 1 , "I totally disagree" (Cronbach's alpha = .93) (Crutzen et al., 2011).

Tailored-processing mechanisms were measured by five concepts. Attention for the tailored advice (e.g. The advice was interesting) was measured by four items on a seven-point 
scale ranging from 7, "I totally agree" to 1 , "I totally disagree" (Cronbach's alpha = .89). Comprehension of the advice (e.g. The advice was clear to me) was measured by four items on a seven-point scale ranging from 7, "I totally agree" to 1, "I totally disagree" (Cronbach's alpha $=.91$ ). Self-reference towards the advice (e.g. The advice was personally relevant for me) was measured by four items on a seven-point scale ranging from 7,"I totally agree" to 1 , "I totally disagree" (Cronbach's alpha $=.91)$. Appreciation of the advice (e.g. I appreciated the advice) was measured by three items on a seven-point scale ranging from 7, "I totally agree" to 1, , I totally disagree" (1) (Cronbach's alpha = .94). Processing of the advice (e.g. The advice encouraged me to think more about smoking cessation) was measured by four items on a seven-point scale ranging from 7, "I totally agree" to 1, "I totally disagree" (1) (Cronbach's alpha $=.93$ ) (Hawkins et al., 2008).

An overall grade for the advice was measured by one item asking respondents to give an overall score for the provided advice from 1 (very bad) to 10 (very good).

\section{Statistical analysis}

First, descriptive analyses were used to determine the sample's characteristics. To test whether any baseline differences existed between the video and text condition, $t$-tests were performed for interval scaled variables, whereas Chi-square tests were conducted for categorical variables. Additionally the same analyses were executed to investigate whether baseline differences existed between highly educated and less educated respondents. Those variables that differed between condition, and or educational levels were included as covariates in all further analyses.

Second, two-way analyses of co-variance (ANCOVA) were carried out to assess whether any differences existed in message-processing mechanisms according to delivery strategy and educational level. The same analyses were executed to detect any differences in future adherence according to delivery strategy and educational level.

Last, a linear regression analysis was conducted to determine the unique predictive power of delivery strategy and educational level on future adherence when user experiences were included as independent predictors in the analysis. All analyses were conducted with SPSS 17.0.

In the main analyses we used the sample of the 139 respondents who stayed more than five minutes on the intervention website. These analyses were repeated using an intention-totreat analysis of the data from all 204 respondents to verify whether we did not introduce a selection bias by our restriction criterion. 


\section{Results}

\section{Sample characteristics}

There were no significant differences between respondents in the video and text condition with regard to gender, educational level, age, cognitive processing, addiction level, and intention to quit smoking.

Respondents in the video condition spent more time on the website in comparison with respondents in the text condition $(t(137)=5.06, p<.001)$, most probably due to the fact that the video condition lasted slightly longer than the text condition. Age of respondents included in the analysis varied from 20 to 72 years (mean 47.39, SD 11.94). Overall, men were slightly underrepresented $(37.4 \%)$. A description of the overall sample at baseline can be found in Table 1.

Table 1 Sample characteristics of adult Dutch smokers ( $N=139)$

\begin{tabular}{|c|c|c|c|c|}
\hline & Overall sample & Text condition & Video condition & $p$ \\
\hline Gender, \% (n) & & & & .42 \\
\hline Female & $62.6(87)$ & $59.5(44)$ & $66.2(43)$ & \\
\hline Male & $37.4(52)$ & $40.5(30)$ & $33.8(22)$ & \\
\hline Educational level, n (\%) & & & & .45 \\
\hline Low & $48.9(68)$ & $45.9(34)$ & $52.3(34)$ & \\
\hline High & $51.1(71)$ & $54.1(40)$ & $47.7(31)$ & \\
\hline Age, mean (SD) & $47.39(11.94)$ & $46.66(11.90)$ & $48.22(12.03)$ & .45 \\
\hline Cognitive processing, M (SD) & $3.56(0.61)$ & $3.55(0.62)$ & $3.57(0.60)$ & .83 \\
\hline FTND score (1-10), M (SD) & $3.57(2.54)$ & $3.41(2.52)$ & $3.75(2.57)$ & .43 \\
\hline Readiness to quit, $\mathrm{n}(\%)$ & & & & .93 \\
\hline Within 1 months & $18.8(26)$ & $19.2(14)$ & $18.5(12)$ & \\
\hline Within 1-3 months & $31.25(43)$ & $30.1(22)$ & $32.3(21)$ & \\
\hline Within 4-6 months & $20.3(28)$ & $19.2(14)$ & $21.5(14)$ & \\
\hline Within 1 year & $17.4(24)$ & $16.4(12)$ & $18.5(12)$ & \\
\hline Within $1-5$ years & $10.9(15)$ & $13.7(10)$ & $33.3(5)$ & \\
\hline After 5 years & $1.4(2)$ & $1.4(1)$ & $1.5(1)$ & \\
\hline $\begin{array}{l}\text { Time spent on website (min), } \\
\text { M (SD) }\end{array}$ & $8.11(12.82)$ & $7.15(9.82)$ & $7.81(16.24)$ & $<.001$ \\
\hline
\end{tabular}

Note: $p$-values $<.05$ are marked bold.

Table 2 shows that respondents with either a high or low educational level did not differ with respect to condition, gender, readiness to quit smoking, and time spent on the website. However, educational level differed significantly with respect to age ( $t(137)=1.90, p=.05)$ and nicotine addiction $(t(136)=4.69, p<.001)$. Lower educated respondents were older and more addicted to nicotine compared to higher educated respondents. Respondents with a higher educational level scored significantly higher on cognitive processing than those with a lower educational level $(t(137)=-5.00, p<.001)$. 
Table 2 Sample characteristics of adult Dutch smokers ( $N=139)$

\begin{tabular}{|c|c|c|c|c|}
\hline & Overall sample & High level & Low level & $p$ \\
\hline Condition, \% (n) & & & & .45 \\
\hline Text & $53.2(74)$ & $56.3(40)$ & $50.0(34)$ & \\
\hline Video & $46.8(65)$ & $43.7(31)$ & $50.0(34)$ & \\
\hline Gender , \% (n) & & & & .39 \\
\hline Female & $62.6(87)$ & $59.2(42)$ & $66.2(45)$ & \\
\hline Male & $37.4(52)$ & $20.0(29)$ & $33.8(23)$ & \\
\hline Age, M (SD) & $47.39(11.94)$ & $45.52(13.63)$ & $49.34(9.60)$ & .05 \\
\hline Cognitive processing, $M(S D)$ & $3.56(0.61)$ & $3.79(0.56)$ & $3.31(0.57)$ & $<.001$ \\
\hline FTND score (1-10), M (SD) & $3.57(2.54)$ & $2.65(2.45)$ & $4.54(2.27)$ & $<.001$ \\
\hline Readiness to quit, \% (n) & & & & .11 \\
\hline Within 1 months & $18.8(26)$ & $23.9(17)$ & $13.4(9)$ & \\
\hline Within $1-3$ months & $31.25(43)$ & $38.0(27)$ & $23.9(16)$ & \\
\hline Within 4-6 months & $20.3(28)$ & $16.9(12)$ & $23.9(16)$ & \\
\hline Within 1 year & $17.4(24)$ & $11.3(8)$ & $23.9(16)$ & \\
\hline Within $1-5$ years & $10.9(15)$ & $8.5(6)$ & $13.4(9)$ & \\
\hline After 5 years & $1.4(2)$ & $1.4(1)$ & $1.5(1)$ & \\
\hline $\begin{array}{l}\text { Time spent on website (min), } \\
M(S D)\end{array}$ & $12.82(8.11)$ & $13.49(9.92)$ & $12.13(5.62)$ & .33 \\
\hline
\end{tabular}

Note: $p$-values $<.05$ are marked bold.

\section{Differences in message-processing mechanisms}

To test possible interaction effects of delivery strategy and educational level on messageprocessing mechanisms, ANOVAs were conducted, using each of the five message-processing measures as dependent variables. For the five measures, no interaction effects were found between delivery strategy and educational level. Subsequently, main effects of delivery strategy were tested on message-processing measures. As shown in Table 3, none of the measures approached significance. Furthermore, main effects of educational level were tested on message-processing measures. As shown in Table 4, lower educated respondents devoted more attention towards the tailored advice compared to higher educated respondents ( $F$ ( 1 , $138)=3.97, p=.05)$. Also, the extent to which respondents processed the information was shown to be higher among lower educated groups $(F(1,138)=4.58, p=.04)$. No differences between lower and higher educated smokers could be found with regard to understanding, self-reference, appreciation, and the overall grade for the advice.

\section{Differences in future adherence}

To test for possible interaction effects of delivery strategy and educational level on future adherence, ANOVAs were conducted, using each of the two measures as dependent variables. For both measures, interaction effects between delivery strategy and educational level were found to be insignificant. Next, main effects of delivery strategy were tested on future adherence. As shown in Table 5, the two measures did not approach significance. Furthermore, main effects of educational level on future adherence were conducted. 
As shown in Table 6, lower educated respondents had a higher intention to visit the website again compared to respondents with a higher educational level $(F(1,138)=4.43, p=.04)$. Recommending the website to others did not differ among lower and higher educated smokers.

Table 3 Differences in variances of delivery strategy with regard to message-processing mechanisms $(N=139)$

\begin{tabular}{llllll}
\hline & Text group & Video group & $F$ & $p$ & $\eta^{2}$ \\
& M (SD) & M (SD) & & & \\
\hline Attention & $4.85(1.43)$ & $4.83(1.28)$ & 0.00 & .97 & .000 \\
Comprehension & $5.50(1.12)$ & $5.41(1.04)$ & 1.15 & .29 & .008 \\
Self-reference & $4.93(1.47)$ & $4.97(1.20)$ & 0.06 & .81 & .000 \\
Appreciation & $5.23(1.43)$ & $5.08(1.32)$ & 0.40 & .53 & .003 \\
Processing & $4.56(1.54)$ & $4.83(1.38)$ & 0.97 & .33 & .007 \\
Grade advice & $7.19(1.36)$ & $6.91(1.56)$ & 1.89 & .17 & .014 \\
\hline
\end{tabular}

Note: $p$-values $<.05$ are marked bold.

Table 4 Differences in variances of socioeconomic status with regard to message-processing mechanisms ( $N=$ 139)

\begin{tabular}{llllll}
\hline & $\begin{array}{l}\text { High level } \\
\text { M (SD) }\end{array}$ & Low level & $F$ & $p$ & $\eta^{2}$ \\
\hline Attention & $4.51(1.46)$ & $5.21(1.14)$ & 3.97 & .05 & .029 \\
Comprehension & $5.41(1.16)$ & $5.54(0.98)$ & 0.02 & .67 & .001 \\
Self-reference & $4.74(1.42)$ & $5.19(1.23)$ & 2.10 & .15 & .016 \\
Appreciation & $4.88(1.50)$ & $5.47(1.16)$ & 1.75 & .19 & .013 \\
Processing & $4.32(1.53)$ & $5.09(1.31)$ & 4.58 & .04 & .033 \\
Grade advice & $6.92(1.46)$ & $7.21(1.54)$ & 1.45 & .23 & .011 \\
\hline
\end{tabular}

Note: $p$-values $<.05$ are marked bold.

Table 5 Differences in variances of delivery strategy with regard to future adherence $(N=139)$

\begin{tabular}{llllll}
\hline & Text group & Video group & $F$ & $p$ & $\eta^{2}$ \\
& $\mathrm{M}(\mathrm{SD})$ & $\mathrm{M}(\mathrm{SD})$ & & & \\
\hline Intention & $4.61(1.58)$ & $4.54(1.61)$ & 0.00 & .95 & .000 \\
Recommendation & $4.65(1.63)$ & $4.74(1.58)$ & 0.03 & .87 & .000 \\
\hline
\end{tabular}

Note: $p$-values $<.05$ are marked bold.

Table 6 Differences in variances of socioeconomic status with regard to future adherence $(\mathrm{N}=139)$

\begin{tabular}{llllll}
\hline & High level & Low level & $F$ & $p$ & $\eta^{2}$ \\
& $\mathrm{M}(\mathrm{SD})$ & $\mathrm{M}(\mathrm{SD})$ & & & \\
\hline Intention & $4.23(1.76)$ & $4.96(1.28)$ & 4.43 & .04 & .032 \\
Recommendation & $4.37(1.72)$ & $5.05(1.41)$ & 2.72 & .10 & .020 \\
\hline
\end{tabular}

Note: $p$-values $<.05$ are marked bold.

\section{Predictors of future adherence}

In order to test whether delivery strategy, educational level, age, smoking dependency, cognitive processing, and time spent on the website were independent predictors of future adherence, multiple regression analysis was executed. User experiences were included as well as independent predictors in the analysis to determine the unique predictive power of delivery 
strategy and educational level in addition to user experiences. Therefore, the first model consisted of user experiences. Second, we investigated whether these results would change after controlling for delivery strategy, educational level, age, gender, and smoking behaviour. Results of the multiple regression analysis are presented in Table 7. User experiences did not alter the observed results.

Table 7 Multiple regression analysis for future adherence $(N=139)$

\begin{tabular}{lll}
\hline $\begin{array}{l}\text { Predictor variable } \\
\text { (Beta) }\end{array}$ & Intention & Recommendation \\
\hline Trustworthiness & -0.145 & -0.093 \\
Enjoyment & -0.057 & -0.171 \\
Active trust & $-0.544^{\mathrm{b}}$ & $-0.487^{\mathrm{b}}$ \\
Design aesthetics & -0.128 & $-0.158^{\mathrm{b}}$ \\
Condition & -0.053 & -0.010 \\
SES & -0.156 & -0.032 \\
Age & -0.086 & 0.017 \\
Gender & -0.061 & -0.028 \\
Smoking dependency & -0.008 & -0.015 \\
Cognitive processing & 0.015 & -0.033 \\
Time on the website & 0.006 & 0.075 \\
R square & 0.697 & 0.721 \\
\hline
\end{tabular}
${ }^{\mathrm{a} p} p<.01 .{ }^{\mathrm{b}} p<.05$.

\section{Intention-to-treat analysis}

The intention-to-treat analysis revealed no different results with regard to sample characteristics. For both, message-processing mechanisms and future adherence interaction effects between delivery strategy and educational level were found to be insignificant. Next, main effects of delivery strategy and educational level were tested on message-processing mechanisms and future adherence. We found a significant difference between the two message methods only regarding the concept of comprehension. Respondents in the text condition showed a higher comprehension of the tailored messages compared to people in the video condition $(F(1,201)=4.34, p=.04)$. However, the results of this analysis should be carefully interpreted since people staying less than five minutes on the website could not possibly have read the advices in-depth.

\section{Discussion}

The first objective of the present study was to investigate whether differences exist in message-processing mechanisms according to delivery strategy and education level for a computer-tailored smoking cessation intervention. Results revealed that delivery strategy did not interact with education on message-processing mechanisms. This means that delivery strategy had no influence on the processing of the message by respondents with different educational levels. Moreover, the processing of the tailored information did not differ between the two conditions. The idea that the processing of computer-tailored information would 
depend on the delivery strategy did not hold for this computer-tailored intervention. This conclusion is in line with results found in a recent study concerning physical activity, which demonstrated no differences between video- and text-generated computer-tailored messages (Vandelanotte et al., 2012). One explanation for these findings may be the exposure time of the intervention. Respondents may have needed to follow more than one session of the intervention in order to detect differences between the two delivery modes. Next, our findings revealed that lower educated smokers paid more attention to the tailored advice and seemed to process information more deeply. As such, lower educated smokers seemed to be more involved in the computer-tailored intervention than higher educated smokers. The findings imply that the current intervention session succeeded in approaching those smokers in the general population who might profit the most from these computer-tailored interventions. Our findings correspond with those from a recent study that found that lower educated respondents were more likely to finish a module of a computer-tailored life style intervention (Schneider et al., 2012). We did not find other differences between educational groups with respect to the other message-processing mechanisms: comprehension, self-reference, and appreciation, which is contrary to previous research that did find higher appreciation rates of computer-tailored advice among lower educated respondents (Brug et al., 2003).

The second objective of this study was to examine whether differences exist in future adherence (intention to revisit, recommend the website to others) with regard to delivery strategy and education. The results revealed no interaction between delivery strategy and education on the intention to adhere; implying that a different delivery strategy did not influence future adherence of lower and higher educated smokers. Again, delivery strategy was found to have no particular effect on future adherence. However, the results demonstrated that lower educated smokers were more inclined to revisit the website compared to higher educated smokers. Although not significant, our findings revealed a slightly better appreciation of the website by lower educated respondents, which may have contributed to the positive intention to revisit the website. As already mentioned, this is in line with findings of a previous study that lower educated respondents were more inclined to initiate a lifestyle program (Schneider et al., 2012). Additionally, our lower educated respondents were slightly less motivated $(p<.11)$ to quit immediately, which also could explain a need for continued help to prepare them to quit in the future. Although lower educated smokers were more inclined to visit the website again, we could not find any educational differences with regard to recommending the website to others. As the results indicate that the "word of mouth" strategy might be not sufficient to recruit respondents for Internet interventions, other recruitment strategies may also be needed such as recruitment through general practitioners (Smit et al., 2011). A question raised by our results is why delivery strategy did not influence message-processing mechanisms and future adherence among different educational groups. One explanation could be that we recruited persons via an Internet 
research agency, which may have more innovative members that have already an open mind to Internet interventions. It might be possible that for those people delivery mode did not differ that much. It is conceivable that delivery mode preference might have been different if we had recruited respondents via other strategies, e.g. newspaper advertisements. Furthermore, it might be possible that respondents were not engaged by the design of the intervention components. The two conditions might have been presented in a more engaging way with further images, graphics, hyperlinks, and other animation effects. However, an important precondition for our experimental design was that the information of the video condition was contingent on the information provided by text condition to reveal the added effect of presenting information through a video format.

Our findings reveal that the given tailored advice was more positively evaluated by lower educated smokers. This outcome is in contrast with earlier studies indicating that webbased programs may result in a digital divide between lower and higher educated groups. Perhaps high Internet use among the Dutch (over 90\%) may explain these effects (CBS, 2011a). Moreover, since our less educated smokers often show higher addiction rates, less fewer quit attempts, and more relapses, (Brouwer et al., 2010, Hiscock et al., 2011) approaching them via Internet may have added potential.

\section{Study limitations}

The present study is subject to certain limitations. First, as with many health communication studies, we could not objectively assess quality of information processing as we could not measure it. Yet, we did assess how long respondents stayed on the website via server registrations and therefore could exclude all respondents who briefly visited the intervention website and probably did not accurately process the given information. Second, our sample size was limited. Additional longitudinal research with a larger sample size is needed to investigate the role of delivery strategy in tailored information and to examine whether smokers will actually revisit the website and whether this will result in the ultimate goal of behaviour change.

\section{Conclusion}

Delivery strategy did not play a role in the processing of the tailored information. Lower educated respondents showed higher attention and processing levels. Lower educated respondents were also more inclined to visit the intervention website again compared to higher educated respondents. Due to the fact that all respondents were members of an Internet research panel, the results can be only applied to people who are already regularly using the Internet. This study can be seen as an important first step to assess the influence of delivery strategy among different educational groups and especially among lower educated 
smokers in the Netherlands. Effective smoking cessation interventions are important to decrease the gap between lower and higher educated smokers. Yet, longitudinal studies with larger sample sizes are needed to see whether these counterintuitive findings still hold true and to further assess additional aspects that we could not assess, such as actual revisit and long-term behavioural effects of the two strategies among lower and higher educated smokers in order to improve computer-tailored smoking cessation interventions. 


\section{CHAPTER 5}

Comparison of text and video computer-tailored interventions for smoking cessation: Randomized controlled trial

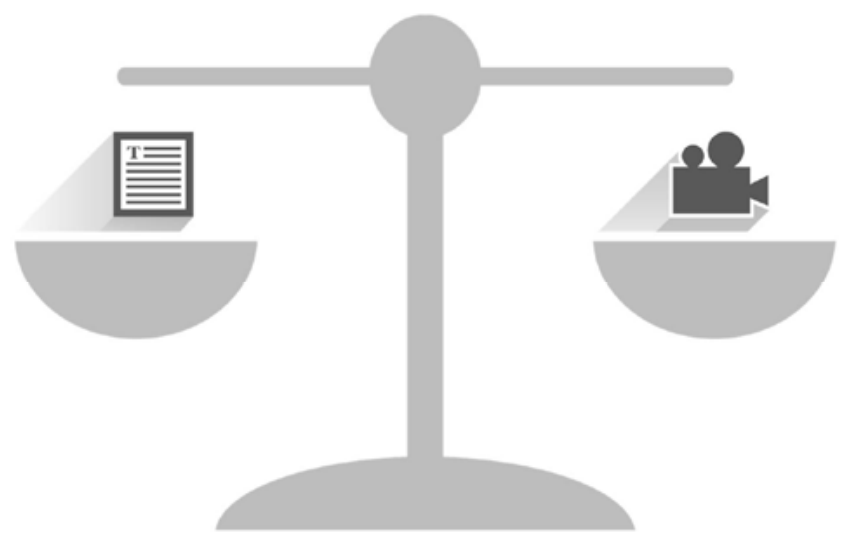

Published as:

Stanczyk, N. E., Bolman, C., Candel, M. J. J. M., van Adrichem, M. J. G., Muris, J. W. M. \& de Vries, H. (2014). Comparison of text and video computer-tailored interventions for smoking cessation: Randomized controlled trial. Journal of Medical Internet Research, 16, e69. 


\begin{abstract}
Background: A wide range of effective smoking cessation interventions have been developed to help smokers to quit. Smoking rates remain high, especially among people with a lower level of education. Multiple tailoring adapted to the individual's readiness to quit and the use of visual messaging may increase smoking cessation.
\end{abstract}

Objective: The results of video computer tailoring (VCT) and text computer tailoring (TCT) were compared with the results of a control condition (CC). Main effects and differential effects for subgroups with different educational levels and different levels of readiness to quit were assessed.

Methods: During a blind randomized controlled trial, smokers willing to quit within six months were assigned to VCT $(n=670)$, TCT $(n=708)$ or to CC (short generic text advice) $(n=721)$. After six months, effects on seven-day point prevalence abstinence and prolonged abstinence were assessed using logistic regression analyses. Analyses were conducted in two samples: (1) respondents (as randomly assigned), who filled in the baseline questionnaire and completed the first session of the program and (2) a subsample of sample 1, excluding respondents who did not adhere to at least one further intervention session. In primary analyses we used a negative scenario in which respondents lost to follow-up were classified as smokers. Complete case analysis and multiple imputation analyses were considered as secondary analyses.

Results: In sample 1, the negative scenario analyses revealed that VCT was more effective in increasing seven-day point prevalence abstinence than CC (OR = 1.45, 95\% Cl 1.09-1.94, $p=$ .01). Next, VCT resulted in significantly higher prolonged abstinence rates than CC among smokers with a low (ready to quit within four to six months) readiness to quit (OR $=5.13,95 \%$ Cl 1.76-14.92, $p=.003$ ). Analyses of sample 2 showed similar results, although TCT was also more effective than $\mathrm{CC}$ in realizing seven-day point prevalence abstinence. No differential effects were found for level of education. Complete case analyses and multiple imputation yielded similar results.

Conclusion: In all analyses, VCT was effective in realizing smoking cessation. Furthermore, VCT was especially successful for smokers with a low readiness to quit smoking. TCT was only effective for sample 2. Results suggest that video-based messages with personalised feedback adapted to the smoker's motivation to quit might be effective in increasing abstinence rates for smokers with diverse educational levels.

Keywords: smoking cessation, multiple computer tailoring, delivery strategy, educational level, text-based messages, video-based messages 


\section{Introduction}

A wide range of different smoking cessation interventions have been developed and implemented. In spite of this smoking rates remain high, especially among people with a lower level of education (Hiscock et al., 2011, Kotz and West, 2009, Nagelhout et al., 2012, Stivoro, 2011b). This illustrates the need to improve smoking cessation intervention strategies for this group. Computer-tailored smoking cessation interventions have already shown to be effective in increasing abstinence rates (Civljak et al., 2013, Elfeddali et al., 2012a, Te Poel et al., 2009). A main characteristic of computer-tailored smoking cessation interventions is that respondents are provided with personalised feedback on their smoking behaviour and motivational characteristics such as attitudes, social support, self-efficacy, intentions and action planning (De Vries and Brug, 1999). Compared to non-tailored information, tailored messages enhance the processing of the health information, are more likely to be read, remembered, and perceived as personally relevant. (Dijkstra, 2005, Kreuter et al., 2003, Spittaels et al., 2007). Additionally, past research has indicated that the effects of tailoring can be enhanced by providing multiple tailored feedback moments (Elfeddali et al., 2012a, Smit et al., 2012) and has suggested a dose-response relationship between the number of feedback moments and smoking abstinence (Borland et al., 2004).

The Internet has become a promising method of delivering smoking cessation interventions and has increased opportunities to reach large numbers of people (Civliak et al., 2010, Shahab and Mc Ewen, 2009). Although web-based computer-tailored smoking cessation interventions have been shown to be potentially effective (Noar et al., 2007, Shahab and Mc Ewen, 2009), they often report problems in attracting, engaging, and retaining smokers and quitters (Balmford et al., 2013, Bennett and Glasgow, 2009, Brouwer et al., 2010, Elfeddali et al., 2012a, Eysenbach, 2005). Smokers with a lower level of education often leave the program before completing all intervention elements and show a lower adherence towards these programs (Moser et al., 2000, Strecher et al., 2008b, Warren-Findlow et al., 2003). Because smokers with a lower level of education appear to be more addicted, show fewer quit attempts, and are often more vulnerable to relapse (Brouwer et al., 2010, Fidler et al., 2008, Kotz and West, 2009), they constitute an important target group for participation in these computer-tailored smoking cessation interventions.

To date, web-based computer-tailored smoking cessation interventions delivered via the Internet often consist of simple text-based messages. However, this might be not attractive enough for Internet users, especially less educated groups (Danaher et al., 2005). Internet users often scan a text for relevant information but do not read the whole text (Liu, 2005, Vandelanotte and Mummery, 2011). Websites increasingly make use of pictures, graphics, and videos, and are often interactive to increase attractiveness (Purcell, 2010). Additionally, previous studies have suggested that the use of rich media, such as videos, may improve the 
appeal of health interventions (Alexander et al., 2010, Frenn et al., 2005, Glasgow, 2007, Tuong et al., 2012) and may attract and stimulate comprehension among low health literacy groups (Campbell et al., 1999, Danaher et al., 2005, Houts et al., 2006). Because video-based information seems to require less mental effort and may help the person to concentrate on the core elements of the message (Guimarães et al., 2000), the use of videos might be a possible strategy to attract, engage, and retain less educated respondents in web-based computer-tailored smoking cessation interventions (Danaher et al., 2005). In contrast, people with a higher level of education might profit more from in-depth processing; therefore, they may be more attracted by text-based messages (Soetens et al., 2014). Studies have already tested the effects of a combination of interactive components, such as graphics, audio-clips and video-clips (Irvine et al., 2004, Swartz et al., 2006, Vandelanotte and Mummery, 2011), to our knowledge no previous study has assessed the specific effect of tailored video-based messages on behavioural change, and in particular, on smoking cessation among groups with different levels of education.

Another strategy to improve the success of computer-tailored smoking cessation interventions is by focusing on the smoker's motivation to quit smoking. Until now, most computer-tailored smoking cessation interventions have been developed for smokers with a high motivation to quit (Civliak et al., 2010, Lancaster and Stead, 2005), whereas less educated smokers often show a lower motivation to quit and might benefit from interventions which give them the possibility and time to reflect on their smoking behaviour and intention to quit and to prepare successfully for their quit attempt. Consequently, web-based computertailored smoking cessation interventions should be adapted to the needs of groups with different levels of education and should take the user's motivation to quit into account.

The study described in this paper was designed to investigate the effectiveness of two multiple computer-tailored smoking cessation interventions after six months: 1) a text-based multiple computer-tailored intervention where smokers received tailored text-based messages during several feedback moments, and 2) a video-based multiple computer-tailored intervention where smokers received tailored video-based messages during several feedback moments. In both interventions, smokers with a high or low readiness to quit were able to choose different routings and received tailored feedback adapted to their readiness to quit. The effectiveness of the two interventions was compared to a control condition (respondents received a generic short text advice).

We hypothesized video-based computer tailoring (VCT) to be more effective in smokers with a lower level of education, whereas text-based computer tailoring (TCT) was expected to be more effective in smokers with a higher level of education. Because the interventions included different routings tailored according to the smokers' readiness to quit, we expected less motivated smokers to be equally successful in their quit attempts as more motivated smokers. Therefore, we explored whether the effects of the two interventions were different 
for individuals with a high or low readiness to quit. Moreover, we conducted our analyses in two different samples: 1) respondents who filled in baseline questionnaire and completed the first session of the program and 2) a subsample of sample 1, excluding respondents who did not adhere to at least one further intervention session (Borland et al., 2004, Elfeddali et al., 2012a). Finally, an overview of the program evaluation of respondents will be shown.

\section{Methods}

The current study was submitted for approval to the Medical Research Ethics Committee (MREC) of Atrium Medical Centre Heerlen. The MREC decided that no MREC approval was necessary because respondents were not required to undertake any particular action. The study was registered at the Dutch Trial Register (NTR3102). The study was in line with the ethical codes of conduct of the American Psychological Association (APA) (American Psychological Association, 2002).

\section{Respondents and recruitment}

Respondents were recruited from December 2010 to June 2012 to participate in the webbased multiple computer-tailored smoking cessation intervention. Respondents were eligible for participation if they were motivated to quit smoking within the next six months, were 18 years or older and had access to the Internet.

Respondents were recruited by several channels. First, a random sample of approximately 150 general practitioners (GPs) was asked to refer smoking patients to the intervention website. The GP practices were provided with recruitment materials (flyers, business cards, etc.) for this purpose. Second, respondents were also recruited to participate through advertising campaigns in local newspapers, newspaper websites, and Dutch health fund websites. Lastly, we used several national and international online social networking websites, such as Hyves and Facebook, to invite smokers to participate in our smoking cessation study. All advertisements provided a link to the intervention website that enabled people to find out more information about the intervention and participation.

\section{Design and procedure}

The current study was a randomized controlled trial with two experimental conditions (TCT vs. VCT) and a control condition ((CC) in which respondents received only a single generic short text advice). Interested respondents could sign up via the intervention website (http://www.steunbijstoppen.nl) (Steunbijstoppen.nl, 2012). On the intervention website, respondents were informed that they could be randomly allocated to one of the three conditions and that they would have the chance to win $€ 100$ if they completed all the assessments (before decision to participate, registration, and baseline measurement). After 
creating a personal login and account, respondents were randomized into one of the three conditions. Respondents were not told about the content of the other experimental condition.

After giving online informed consent, respondents were asked to fill out the baseline questionnaire. Respondents in the text-based and video-based condition received tailored feedback over three months (see Intervention description and Figure 1 for details). At sixmonth follow-up, all respondents were sent an e-mail invitation with a link to the intervention website to fill out the six-month follow-up measurement. Respondents who did not complete the follow-up measurement after one week were reminded by e-mail to fill out the online questionnaire. A further reminder was sent after two weeks if necessary. Respondents who did not respond to the e-mail invitation or the two reminders received another e-mail, inviting them to briefly indicate their current smoking status. This e-mail requested completion of a shortened version of the six-month follow-up measurement, consisting of 10 (instead of 95) important smoking-related questions, which they could return by e-mail. Lastly, if this abbreviated e-mail assessment was still not completed, respondents were called for a short telephone interview, asking the same questions as in the shortened online questionnaire.

\section{Intervention}

The two web-based multiple computer-tailored smoking cessation interventions (TCT vs. VCT) varied only in their mode of delivery (see Figure 1). The intervention was based on two previously tested computer-tailored interventions which were found to be effective in smoking cessation (Smit et al., 2012, Te Poel et al., 2009). The I-Change model, integrating various social cognitive theories (De Vries et al., 2003, De Vries et al., 2013, De Vries et al., 2008), was used as a theoretical framework of the currently tested intervention. After completing the baseline assessment, respondents in the two experimental conditions first received tailored feedback on their smoking behaviour, followed by feedback about their attitude (pros and cons toward smoking and quitting), their perceived social influence (modeling and support), their perceived self-efficacy and how to prepare to quit (e.g. how to plan a quit date). Next, respondents were asked whether they wanted to quit within a month. Depending on this readiness to quit smoking within the following month, respondents received more personalised feedback during subsequent multiple computer-tailored sessions, defined in routing 1 or 2 . These routings varied between those who already wanted to set a quit date within a month compared to those who did not plan a quit date in the forthcoming month (see Figure 1). Respondents in the two experimental conditions (TCT vs. VCT) also received an overview of the tailored advice by email after each session. 


\section{Routing 1}

Respondents who had set the goal to quit within one month were directed to routing 1 . The goal of routing 1 was to help smokers translate their intention to quit into action by providing tailored feedback to increase self-efficacy and effective action planning. In the first session, after receiving feedback on their smoking behaviour, attitude, social influences and selfefficacy with respect to quitting, respondents were asked to choose a quit date (between eight days and one month from the first session). At the end of this first session, respondents were informed that they would be invited to the next session one week before their quit date, to receive help with quitting. During the second session (one week before their quit attempt), respondents received feedback on the extent to which they had already made concrete plans for their quit attempt, because past research has revealed that preparing for quitting increases the likelihood of quitting (Te Poel et al., 2009). In addition, they received feedback on their perceived self-efficacy, including tailored feedback on coping planning to help them to deal with difficult situations that may cause relapse. Different studies have found that ex-smokers often relapse shortly after their quit attempt; therefore, respondents were provided with these different relapse prevention strategies as described previously (De Vries et al., 2013, Elfeddali et al., 2012b, Van Osch et al., 2008). During the third session, three days after their quit attempt, feedback was given on the quitter's perceived self-efficacy. Respondents also received personalised tips on how to deal with personal risk situations and were invited to formulate coping plans again to prevent potential relapse. During the fourth session, two weeks after their quit date, respondents received tailored feedback on their perceived selfefficacy, including feedback on how to deal with difficult situations and attitude toward smoking and quitting (perceived pros and cons of smoking and quitting). In session five and six, four and eight weeks, respectively, after their quit date, a similar strategy was used as for session four. Respondents could choose to receive feedback on different items (e.g. how to cope with negative moods (coping plans) or how to deal with difficult risk situations (selfefficacy)). This option was provided because we expected respondents to encounter different problems throughout their quit attempt. During all sessions, respondents were invited to continue their quit attempt or, if they had relapsed, to indicate their readiness to quit smoking and plan a new quit date. Respondents could restart their quit attempt several times (no maximum) during the program, if they wanted. 


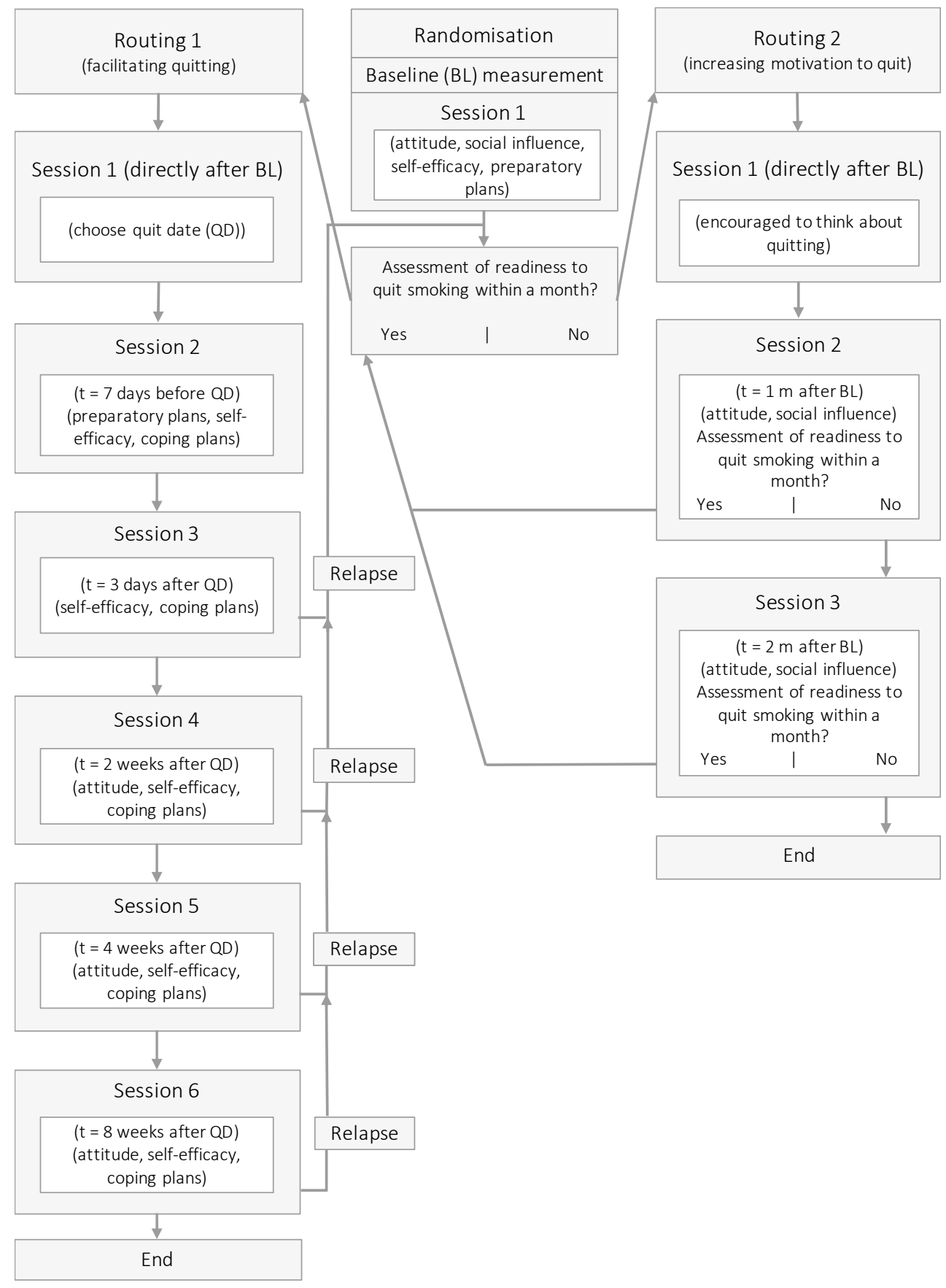

Figure 1 Intervention design of a video- and text-based computer-tailored intervention for smoking cessation, following two routings 


\section{Routing 2}

Respondents who were not ready to quit within one month were directed to routing 2 . The goal of routing 2 was to increase motivation by increasing perception of the pros of quitting and knowledge of how to obtain support for quitting. In session one, directly after completion of the baseline assessment, smokers were encouraged to use the following month to reflect on their smoking behaviour and motivation to quit. In session two, one month after baseline, respondents were invited by e-mail for the next session. Respondents received tailored feedback on their smoking behaviour, their attitude (pros and cons of smoking and quitting) and their perceived social support. Next, they were invited to indicate their readiness to quit smoking. Respondents who indicated an intention to quit within one month were directed to routing 1 and were asked to set a quit date. Respondents who were not ready to quit received an invitation to take part in the next session (session three); this session used a similar strategy that was used in session two. Respondents ready to quit were directed to routing 1 and were asked to set a quit date. Respondents who indicated at the end of session three that they were not prepared to quit received a kind message, indicating that the intervention program would respect the fact that they were not ready to quit smoking and that they would receive no further invitations.

\section{Mode of delivery}

The content of the feedback messages was exactly the same in both the text- and video-based conditions. In the text-based condition, respondents received multiple sessions of text-based computer-tailored advice without any graphics or animations. In the video-based condition, the same tailored advice was presented by adults in a video message. Five different adult presenters (two males, three females) were selected out of a screening test of 20 persons who delivered the tailored advice in a TV 'news program' format. We used a mix of adults during the different sessions who presented the different pieces of tailored advice.

\section{Measurements}

\section{Baseline measurement}

The following demographic variables were assessed: age, gender ( 0 = male; $1=$ female), educational level ( 1 = low (corresponding to primary, basic vocational, lower general school, or no education); 2 = intermediate (corresponding to higher general secondary education, preparatory academic education, or medium vocational school); 3 = high (corresponding to higher vocational school or university level) $)$ and nationality $(0=$ other nationality; $1=$ Dutch nationality).

Addiction level was measured by six items using the Fagerström Test for Nicotine Dependence (FTND), asking respondents how many cigarettes they smoked per day, at which 
time points, and whether they had difficulties not to smoke in smoke-free places $(0=$ not addicted; 10 = highly addicted) (Fagerström, 2012).

Readiness to quit smoking was assessed with a single item asking respondents whether and when they intended to quit smoking, resulting in three categories ( $1=$ yes, within four to six months; 2 =yes, within one to three months; 3 = yes, within the following month) (De Vries et al., 2008).

Smoking habit was assessed using an abbreviated version of Verplanken and Orbell's Self-Reported Habit Index of six items (e.g. Smoking is something which I do automatically) with which respondents could agree or disagree, resulting in a five-point scale ( $1=$ I totally disagree; $5=$ I totally agree). A mean scale score was included in the analyses (Cronbach's alpha $=.78$ ) (Verplanken and Orbell, 2003).

Depressive symptoms were measured by the abbreviated CES-D by four items that asked respondents whether they felt depressed during the past week, for example, resulting in a four-point scale ( 1 = rarely or none of the time; $4=$ most or all of the time) (Cole et al., 2004). A sum score was included in the analyses (Cronbach's alpha $=.85$ ).

Occurrence of smoking-related diseases was measured by four questions on a dichotomous scale (Do you suffer from chronic obstructive pulmonary disease (COPD), cancer, diabetes or cardiovascular disease?) ( 0 = no; 1 = yes)

Attitude was measured by three items assessing the pros and cons of quitting (quitting smoking would be reasonable, bad or enjoyable), resulting in a five-point scale ( $1=$ I totally disagree; 5 = I totally agree). A mean scale score was included in the analyses (Cronbach's alpha $=.52$ ). A higher score represents a positive attitude toward quitting.

Social influence was measured by two scales: a social modelling and a social support scale. Social modelling was assessed by two items that measured whether other people in the environment smoked (respectively partners ( $1=$ no; $2=$ yes; $9=$ not applicable) and social environment (e.g. family or friends; 1 = none; 2 = a minority; 3 = half; 4 = a majority; 5 = all; 9 $=$ not applicable)). A total of 552 respondents for the partner question and 80 respondents for the social environment question filled in 'not applicable' when they were asked whether their partner or their social environment smoked. Social support was measured by two items that asked whether smokers received social support (partners and social environment, respectively) in favour of quitting on a four-point scale ( 1 = no; 2 =yes, a bit; 3 = yes, moderate; 4 =yes, a lot; $9=$ not applicable). A total of 787 respondents for the partner question and 229 for the social environment question filled in 'not applicable' when they were asked whether they received support from their partner or their social environment. Not applicable was recoded into the lowest value ( 1 = no support) for the social influence measure. The items were summed and formed an index that was included in the analyses.

Preparatory plans were assessed by three items that measured whether respondents planned to execute different preparatory plans for their quit attempt (removing ashtrays, 
telling their environment to quit smoking, quitting without decreasing smoking first) on a fivepoint scale ( 1 = surely not; 5 = surely yes). The items were summed and formed an index that was included in the analyses.

Coping plans were assessed by four items that measured whether respondents had made specific plans to prevent relapse in difficult situations (such as plans how to cope with negative mood, plans how to cope when being at a party or drinking a cup of coffee, or being offered a cigarette $(0=$ no; $1=$ yes $))$. Difficult situations were selected and predefined based on previous studies (Elfeddali et al., 2012b, Smit et al., 2012, Te Poel et al., 2009). The items were summed and formed an index that was included in the analyses.

Self-efficacy was measured by three items asking respondents whether they would be able to refrain from smoking in these difficult situations (Do you think you will manage not to smoke when you drink a cup of coffee, when you are in a negative mood, or when you visit a party?), resulting in a five-point scale ( 1 = definitely not; $5=$ yes, definitely). A mean scale score was included in the analyses (Cronbach's alpha $=.62$ ).

The variables attitude, self-efficacy, preparatory plans and coping plans were also used to determine the tailored advice during the first session of the intervention.

\section{Follow-up measures}

At the six-month follow-up measurement, seven-day point prevalence abstinence was selfassessed by one item asking respondents whether they had refrained from smoking during the past seven days ( $0=$ no; 1 = yes) (Hughes et al., 2004, Mudde et al., 2006).

In addition, prolonged abstinence was self-assessed by one item asking respondents whether they had refrained from smoking since their last quit attempt (allowing for a twoweek grace period during which the respondent could smoke one to five cigarettes) ( $0=$ no; 1 = yes) (Hughes et al., 2004, Mudde et al., 2006). In line with the definition of prolonged abstinence, those who reported that they had quit less than three months before the followup measurements were not included as quitters in the prolonged abstinence measurement (Hughes et al., 2004).

\section{Evaluation of the program}

Process evaluation was conducted by measuring five concepts, each measured on a five-point scale ( $1=$ totally disagree to 5 = totally agree).

Attention to the tailored advice (e.g. The advice was interesting) was measured by three items (Cronbach's alpha $=.94)$.

Comprehension of the advice (e.g. The advice was clear to me) was measured by three items (Cronbach's alpha $=.78$ ).

Adaptation towards the advice (e.g. The advice was personally relevant for me) was measured by three items (Cronbach's alpha $=.79$ ). 
Appreciation of the advice (e.g. I appreciated the advice) was measured by three items (Cronbach's alpha $=.93$ ).

Processing of the advice (e.g. The advice encouraged me to think more about smoking cessation) was measured by eight items. For all process evaluation scales a mean scale score was included in the analyses (Cronbach's alpha $=.91$ ).

\section{Statistical analysis}

The inclusion of all randomly assigned respondents is a common approach to analyse the effects of an intervention (Heritier et al., 2003, Hollis and Campbell, 1999). Because not all respondents (video and text conditions) adhered to all intervention elements, the inclusion of these respondents in the effect analyses might distort the assessment of an intervention's effectiveness. It might be adequate to include only respondents in the analyses who actually followed the intervention for at least one session (Elfeddali et al., 2012a). Therefore, we chose to analyse two different samples. The first sample included all randomly assigned respondents that filled in baseline questionnaire and session 1 (directly after baseline assessment, including setting a quit date). The second sample included only respondents in the experimental conditions who at least finished one further session of the two different routings of the intervention.

As a preliminary, descriptive analyses were conducted to check for baseline differences between the three conditions. Chi-square tests were used for categorical variables whereas analyses of variance (ANOVAs) were used for continuous variables. If the Chi-square test showed a $p$-value $<.05$, post-hoc pairwise comparisons with Bonferroni correction (alpha $=$ $.05 / 3=.017$ ) were used. If the overall F-test showed a $p$-value $<.05$, the Tukey-HSD (Honestly Significant Difference) method was used for post-hoc pairwise comparisons. Second, logistic regression was used to analyse attrition, including baseline factors and condition as predictors. Baseline differences and significant predictors of dropout were included in all logistic regression effect analyses explained subsequently. Third, logistic regression analyses were conducted to investigate the effectiveness of the intervention on the outcome measures assessed at the six-month follow-up measurements. The analyses were performed adjusting for potential confounders, including demographic variables (e.g. age, educational level, gender and ethnicity) and possible moderators of the intervention effect (e.g. addiction level, recruitment strategy, readiness to quit smoking, depression, smoking-related illnesses, selfefficacy, preparatory planning and coping planning), baseline differences, drop-out predictors and two interaction terms (readiness to quit smoking by condition and educational level by condition). Where significant interaction terms were found, stratified analyses were performed separately for each group.

In the effect analyses a negative scenario was used in which every respondent missing at follow-up was regarded as a smoker. In addition, we also used multiple imputation (Schafer, 
1999) to fill in missing values. Missing values for outcome variables were imputed based on the regression of all relevant variables that were used in the main effect analyses. The number of imputations was set at 30 . This was done according to the recommendation to create as many imputed datasets as the percentage of cases with missing data (Bodner, 2008).

Lastly, we also conducted complete case analyses, in which we only took respondents into account who filled out the six-month follow-up measurements (these results are presented in the Appendix).

Data were analysed using SPSS 19.0 (SPSS, Inc., Chicago, IL, USA).

\section{Results}

\section{Sample characteristics and attrition analysis}

Figure 2 shows the flow of respondents for the three conditions. Of the 2,551 potential respondents who were randomized to one of the three conditions, overall 49 (1.9\%) declined to participate, 138 (5.4\%) did not meet inclusion criteria and 265 (10.4\%) did not complete the baseline questionnaire or had no baseline quit date (within routing 1). As Figure 2 illustrates eligibility was checked after randomization. The different tailored feedback sessions were organized around the quit date; therefore, respondents had to fill in their quit date (in the experimental conditions) otherwise they were excluded from the study. Finally, 2,099 respondents were included to the VCT condition $(n=670)$, TCT condition $(n=708)$, and the CC $(n=721)$.

Table 1 shows the characteristics of the total sample and the baseline differences between the three conditions in terms of demographic and smoking-related variables. Mean age of respondents was 45.7 years (SD 12.8). Of the 2,099 respondents, 1,278 (60.9\%) of them were female and 705 (33.6\%) had a low level of education. Furthermore, respondents smoked on average approximately 19 (SD 8.6) cigarettes per day. Respondents in the three conditions differed significantly in terms of readiness to quit smoking. Respondents in the VCT and TCT conditions were less ready to quit smoking than respondents in the CC. Moreover, there were also differences between the three conditions regarding preparatory planning and coping planning. Respondents in the two experimental conditions were more likely to have made preparatory and coping plans compared to respondents in the CC. In sample 1, 238 of 670 (35.5\%) were lost to follow-up in the VCT condition, versus 212 of 708 (29.9\%) in the TCT condition versus 196 of 721 (27.2\%) in the CC. Attrition analysis showed that respondents were significantly more likely to complete the follow-up assessment if they were in the TCT and CC ( $O R=1.32,95 \% \mathrm{Cl} 1.05-1.67, p=.02 ; \mathrm{OR}=1.47,95 \% \mathrm{Cl} 1.16-1.87, p=.001$, respectively), if recruited by Internet or Newspaper advertisements ( $O R=0.62,95 \% \mathrm{Cl} 0.40-0.97, p=.04)$, if they were older $(\mathrm{OR}=1.02,95 \% \mathrm{Cl} 1.01-1.03, p=.001)$, were of Dutch nationality $(\mathrm{OR}=1.63$, $95 \% \mathrm{Cl} 1.06-2.52, p=.03)$, and had higher levels of self-efficacy $(\mathrm{OR}=1.12,95 \% \mathrm{Cl}$ 1.06-1.33, 
$p=.003)$. Baseline differences and significant predictors at dropout were included in all further analyses as potential confounders.

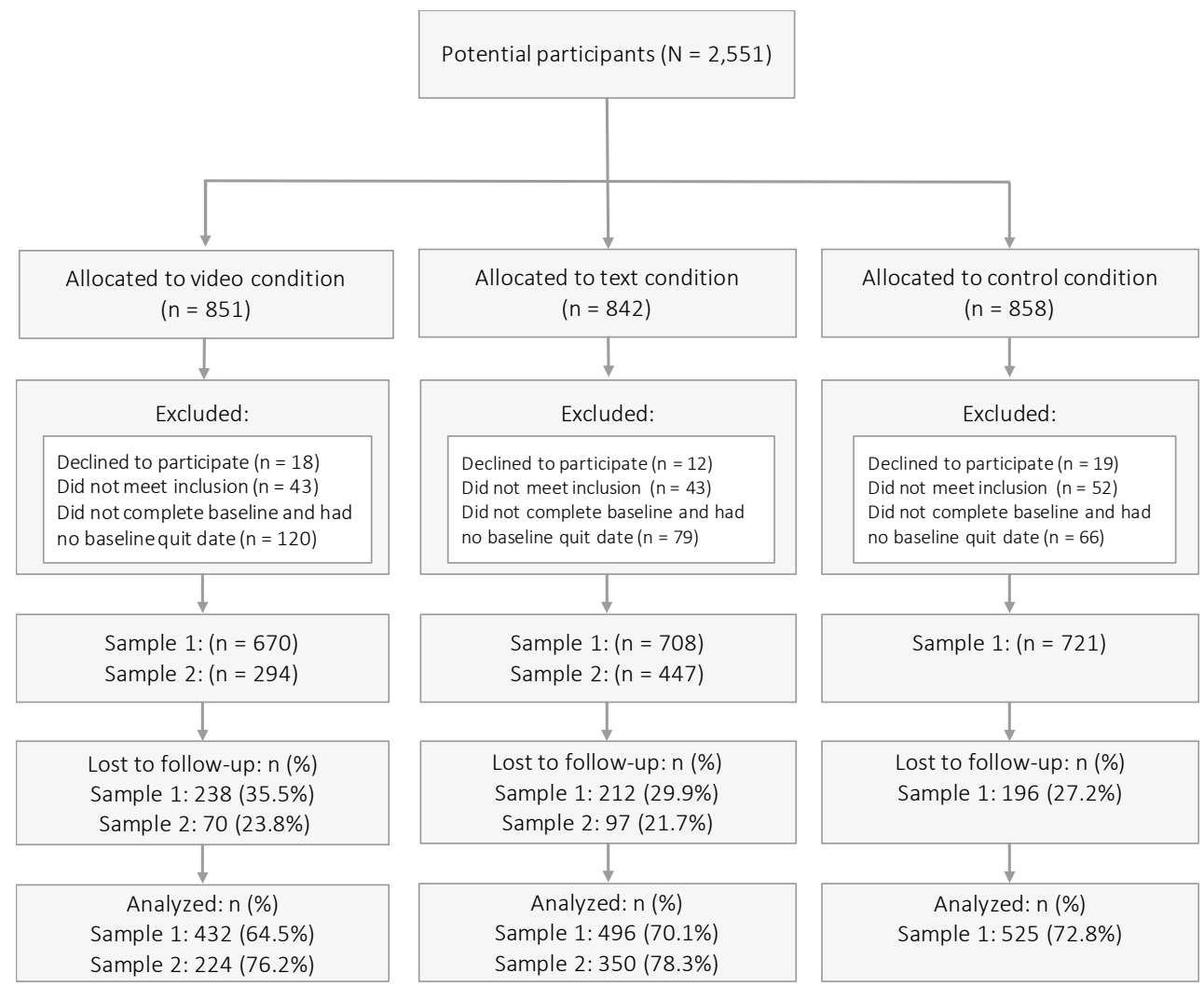

Figure 2 Flowchart of respondent's enrolment and inclusion

\section{Quit rates at six-month follow-up}

Table 2 shows the raw abstinence rates for sample 1 for the negative scenario. When respondents lost to follow-up were regarded as smokers (negative scenario), seven-day point prevalence abstinence rates in sample 1 were $20.9 \%$ in the VCT condition, $17.9 \%$ in the TCT condition and $14.6 \%$ in the CC. In sample 2 , seven-day point prevalence abstinence rates were $30.6 \%$ in the VCT condition, $22.6 \%$ in TCT condition and $14.6 \%$ in the CC (see Appendix 1, Table 1). Prolonged abstinence rates are also presented stratified by readiness to quit smoking. In sample 1, prolonged abstinence rates in the VCT, TCT and the CC (for people with a lower readiness to quit (four to six months)) were $14.4 \%, 8.3 \%$, and $3.3 \%$, respectively. Finally, sample 2 showed prolonged abstinence rates (for people with a lower readiness to quit (within four to six months)) of $23.1 \%, 9.5 \%$, and, $3.3 \%$ in the VCT condition, TCT condition and the CC (see Appendix 1, Table 1). 


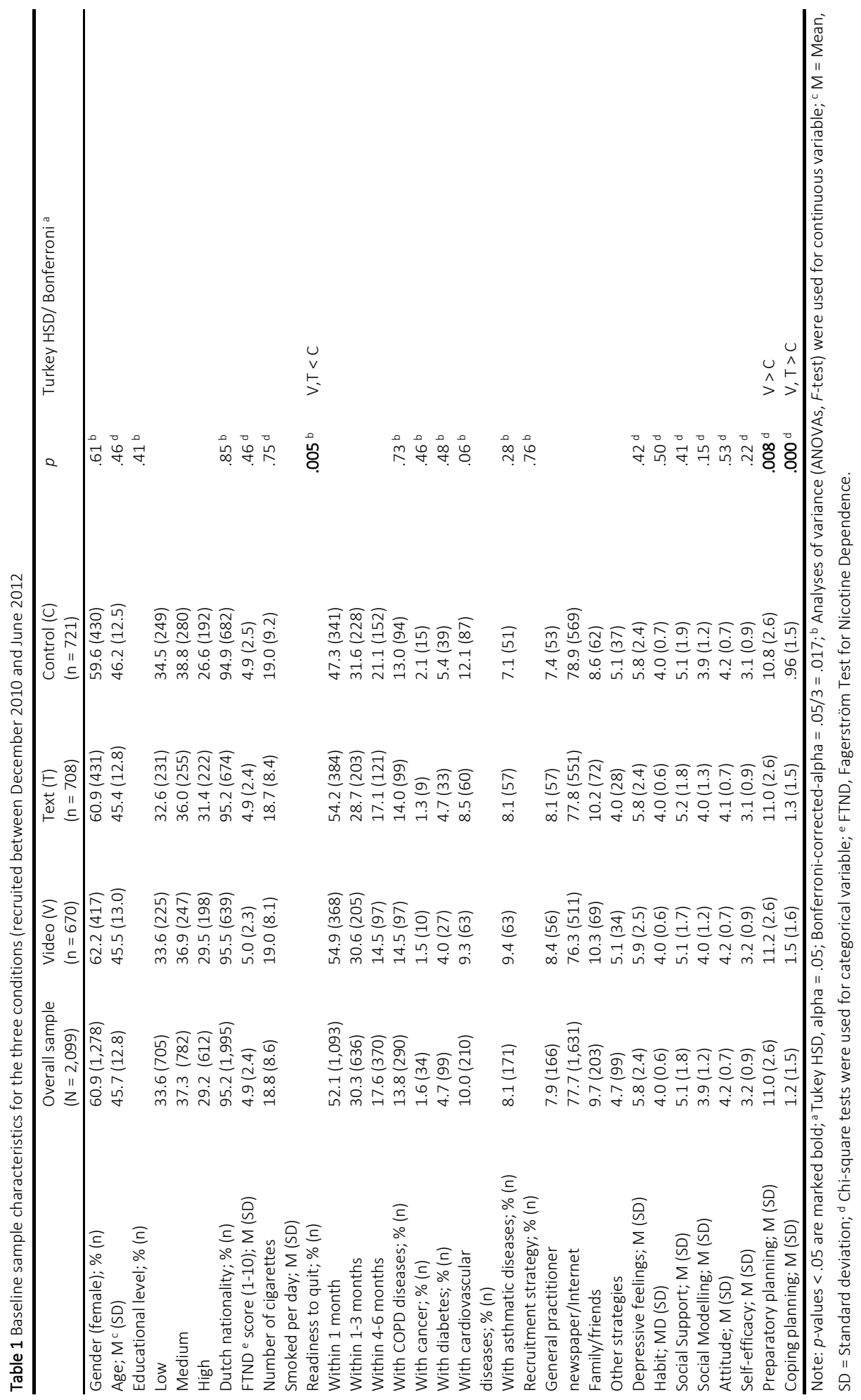


Table 2 Six-month abstinence rates (Seven-day point prevalence abstinence and prolonged abstinence) per condition for sample 1 (negative scenario)

\begin{tabular}{|c|c|c|c|c|c|}
\hline Negative scenario & Total $(\mathrm{N})$ & Video & Text & Control & $p$ \\
\hline Seven-day PPA ${ }^{a} ; \%(n)$ & 2,099 & $20.9(140)$ & $17.9(127)$ & $14.6(105)$ & .008 \\
\hline$P A$ b; $\%(n)$ & 2,099 & $14.6(98)$ & $14.0(99)$ & $12.1(87)$ & .34 \\
\hline $\mathrm{PA}{ }^{\mathrm{b}} ; \%(\mathrm{n})$ & 2,099 & & & & \\
\hline \multicolumn{2}{|c|}{ Readiness to quit within 1 month } & $16.8(62)$ & $18.5(71)$ & $15.2(52)$ & .51 \\
\hline \multicolumn{2}{|c|}{ Readiness to quit within $1-3$ months } & $10.7(22)$ & $8.9(18)$ & $13.2(30)$ & .36 \\
\hline \multicolumn{2}{|c|}{ Readiness to quit within $4-6$ months } & $14.4(14)$ & $8.3(10)$ & $3.3(5)$ & .006 \\
\hline
\end{tabular}

Note: $p$-values < .05 are marked bold; ${ }^{a}$ Point prevalence abstinence (PPA); ${ }^{\text {b }}$ Prolonged abstinence (PA).

\section{Differences in seven-day point prevalence abstinence between conditions at follow-up}

When respondents lost to follow-up were regarded as smokers in the analyses, no significant interaction was found between the type of condition and educational level $\left(\chi^{2}=6.30, \mathrm{df}=4, p\right.$ $=.18$ ) nor between condition and respondents readiness to quit smoking $\left(\chi^{2}=3.14, \mathrm{df}=4, p=\right.$ .54) on seven-day point prevalence abstinence. Our analysis, however, revealed a main intervention effect on seven-day point prevalence abstinence. In sample 1 (including all respondents as randomly assigned), VCT was significantly more effective than the CC (OR = $1.45,95 \% \mathrm{Cl} 1.09-1.94, p=.01$ ). In sample 2 (with only those who followed at least one further session of the intervention), both experimental conditions were significantly more effective than the CC (VCT: OR = 2.29, 95\% Cl 1.64-3.20, $p=.000$; TCT: OR = 1.57, 95\% Cl 1.15-2.15, $p=$ .005) (see Appendix 1, Table 2). Next to this, in sample 2, the VCT condition was significantly more effective than the TCT condition ( $\mathrm{OR}=1.46,95 \% \mathrm{Cl} 1.04-2.05, p=.03)$. Other predictors of seven-day point prevalence abstinence were a higher readiness to quit, a lower degree of depressive symptoms, making more preparatory plans, having a higher self-efficacy, and being recruited by GPs (see Table 3). The multiple imputation procedure revealed similar results. In samples 1 and 2 again VCT was significantly more effective than the CC (sample 1: OR $=1.55$, $95 \% \mathrm{Cl} 1.16-2.08, p=.003$; sample 2: OR $=1.93,95 \% \mathrm{Cl} 1.38-2.70, p=.000)$. The TCT condition in sample 2 did not reach significance when compared to the CC (TCT: OR $=1.31,95 \% \mathrm{Cl} 0.96$ $1.78, p=.09$ ). Complete case analyses revealed comparable results (see Appendix 2, Table 1). 
Table 3 Factors associated with seven-day point prevalence abstinence in sample 1 (negative scenario) in the present study

\begin{tabular}{|c|c|c|c|}
\hline \multirow{2}{*}{$\begin{array}{l}\text { Negative scenario } \\
\text { Variable }\end{array}$} & \multicolumn{3}{|c|}{ Sample $1(N=2,099)$} \\
\hline & $\mathrm{OR}$ & $95 \% \mathrm{Cl}$ & $p$ \\
\hline VCT vs. CC & 1.45 & $1.09-1.94$ & .01 \\
\hline TCT vs. CC & 1.22 & $0.92-1.63$ & .17 \\
\hline Gender (male) & 0.90 & $0.70-1.14$ & .38 \\
\hline Age & 1.01 & $1.00-1.02$ & .07 \\
\hline Dutch nationality & 1.23 & $0.71-2.15$ & .46 \\
\hline Middle education level ${ }^{a}$ & 1.08 & $0.81-1.45$ & .58 \\
\hline High education level a & 1.17 & $0.87-1.59$ & .30 \\
\hline Readiness to quit within 1 month ${ }^{b}$ & 1.71 & $1.16-2.50$ & .006 \\
\hline Readiness to quit within $1-3$ months $^{b}$ & 1.41 & $1.17-2.10$ & .09 \\
\hline FTND score & 0.96 & $0.91-1.00$ & .07 \\
\hline CES-D score & 0.94 & 0.89-.99 & .03 \\
\hline With COPD ${ }^{\mathrm{c}}$ & 1.03 & $0.71-1.50$ & .86 \\
\hline With cancer ${ }^{c}$ & 1.00 & $0.40-2.50$ & .99 \\
\hline With diabetes ${ }^{c}$ & 1.22 & $0.69-2.20$ & .51 \\
\hline With cardiovascular diseases ${ }^{c}$ & 1.18 & $0.78-1.78$ & .43 \\
\hline With asthma ${ }^{c}$ & 0.89 & $0.58-1.39$ & .61 \\
\hline Recruitment strategy & 0.67 & $0.45-.99$ & .04 \\
\hline \multicolumn{4}{|l|}{ Newspaper/Internet ${ }^{d}$} \\
\hline Preparatory planning & 1.07 & $1.02-1.12$ & .009 \\
\hline Coping planning & 1.01 & $0.94-1.10$ & .72 \\
\hline Self-efficacy & 1.15 & $1.01-1.33$ & .04 \\
\hline
\end{tabular}

Note: $p$-values < .05 are marked bold; Interaction terms are not included in the final model since they were not significant and ORs are adjusted for variables significant at baseline and drop-out; ${ }^{a}$ Low education is the reference category; ${ }^{b}$ Willingness to quit within 4-6 months is the reference category; ${ }^{c}$ Not suffering from the disease is the reference category; ${ }^{d}$ General practitioner (GP) is the reference category.

\section{Differences in prolonged abstinence between conditions at follow-up}

In the negative scenario, no significant interaction was found between condition and educational level on prolonged abstinence $\left(\chi^{2}=3.12, \mathrm{df}=4, p=.54\right)$. However, analysis revealed a significant interaction effect between the type of intervention and respondents readiness to stop smoking with regard to prolonged abstinence in sample 1 ( $\chi^{2}=11.98, \mathrm{df}=4$, $p=.02$ ). Subsequent subgroup analysis showed that VCT was significantly more effective than the $\mathrm{CC}$ in increasing prolonged abstinence rates among respondents who were less motivated to quit, i.e. those who had stated they were ready to quit within four to six months (see subgroup analyses in Table 4). Similarly, in sample 2, a significant interaction was found between the type of intervention and respondents readiness to quit smoking with regard to prolonged abstinence $\left(\chi^{2}=10.55, \mathrm{df}=4, p=.03\right)$. Subsequent subgroup analysis showed that VCT was more effective in increasing prolonged abstinence rates among respondents with high (within one month) and low (within four to six months) readiness to quit (see Appendix 1, Table 3). The multiple imputation analyses yielded similar results. A significant interaction was shown, in which the VCT condition was still effective for smokers who were ready to quit within four to six months in both samples (Sample 1: OR = 2.75, 95\% Cl 0.94-8.10, $p=.06$; Sample 2: 
$\mathrm{OR}=3.17,95 \% \mathrm{Cl}$ 0.94-10.73, $p=.06)$. The complete case analyses yielded similar results compared to the other analyses (see Appendix 2, Table 2).

Table 4 Factors associated to prolonged abstinence in sample 1 (negative scenario) in the present study

\begin{tabular}{|c|c|c|c|}
\hline \multirow{2}{*}{$\begin{array}{l}\text { Negative scenario } \\
\text { Variables }\end{array}$} & \multicolumn{3}{|c|}{ Sample 1} \\
\hline & OR & $95 \% \mathrm{Cl}$ & $p$ \\
\hline VCT vs. CC & 5.13 & $1.76-14.92$ & .003 \\
\hline TCT vs. CC & 2.79 & $0.92-8.46$ & .07 \\
\hline Gender (male) & 0.72 & $0.54-0.95$ & .02 \\
\hline Age & 1.01 & $1.00-1.02$ & .05 \\
\hline Dutch nationality & 1.31 & $0.69-2.44$ & .40 \\
\hline Middle Education level ${ }^{a}$ & 1.16 & $0.84-1.61$ & .35 \\
\hline High education level a & 1.01 & $0.72-1.43$ & .94 \\
\hline Readiness to quit within 1 month ${ }^{b}$ & 4.18 & $1.61-10.85$ & .003 \\
\hline Readiness to quit within $1-3$ months $^{b}$ & 4.11 & $1.55-10.95$ & .005 \\
\hline FTND score & 0.95 & $0.89-1.00$ & .04 \\
\hline CES-D score & 0.90 & $0.84-0.96$ & .002 \\
\hline With COPD ${ }^{c}$ & 1.23 & $0.80-1.89$ & .34 \\
\hline With cancer ${ }^{c}$ & 0.66 & $0.26-1.66$ & .37 \\
\hline With diabetes ${ }^{c}$ & 1.04 & $0.55-1.96$ & .90 \\
\hline With cardiovascular diseases ${ }^{c}$ & 1.24 & $0.78-1.89$ & .37 \\
\hline With asthma ${ }^{c}$ & 1.12 & $0.67-1.90$ & .66 \\
\hline Recruitment strategy & .62 & $0.41-0.95$ & .03 \\
\hline \multicolumn{4}{|l|}{ Newspaper/Internet ${ }^{d}$} \\
\hline Preparatory planning & 1.08 & $1.02-1.14$ & .007 \\
\hline Coping planning & 1.07 & $0.98-1.17$ & .14 \\
\hline Self-efficacy & 1.18 & $1.01-1.38$ & .04 \\
\hline \multicolumn{4}{|l|}{ Interaction } \\
\hline High readiness to quit $*$ VCT & 0.21 & $0.07-0.66$ & .007 \\
\hline High readiness to quit $*$ TCT & 0.45 & $0.12-1.45$ & .18 \\
\hline Middle readiness to quit $* \mathrm{VCT}$ & 0.15 & $0.04-0.51$ & .002 \\
\hline Middle readiness to quit $*$ TCT & 0.23 & $0.06-0.81$ & .02 \\
\hline \multicolumn{4}{|l|}{ Subgroup analyses } \\
\hline \multicolumn{4}{|l|}{ Readiness to quit within 1 month } \\
\hline VCT vs. TCT & 0.86 & $0.59-1.27$ & .46 \\
\hline VCT vs. CC & 1.07 & $0.71-1.62$ & .74 \\
\hline TCT vs. CC & 1.24 & $0.83-1.86$ & .29 \\
\hline \multicolumn{4}{|l|}{ Readiness to quit within 1-3 months } \\
\hline VCT vs. TCT & 1.23 & $0.63-2.40$ & .54 \\
\hline VCT vs. CC & 0.77 & $0.43-1.41$ & .40 \\
\hline TCT vs. CC & .63 & $0.34-1.18$ & .15 \\
\hline \multicolumn{4}{|l|}{ Readiness to quit within 4-6 months } \\
\hline$\overline{\text { VCT vs. TCT }}$ & 1.84 & $0.77-4.40$ & .17 \\
\hline VCT vs. CC & 5.13 & $1.76-14.92$ & .003 \\
\hline TCT vs. CC & 2.80 & $0.92-8.46$ & .07 \\
\hline
\end{tabular}




\section{Adherence to the intervention}

Table 5a presents the seven-day point prevalence abstinence rates for the two experimental conditions. Results showed significant higher abstinence rates for higher educated smokers in the text condition. Table $5 b$ shows the seven-day point prevalence abstinence rates for those who did and who did not adhere to at least one further intervention element.

Table 5a Abstinence rates per educational level for the video-based and text-based computer-tailored interventions

\begin{tabular}{llll}
\hline & Abstinent $\mathrm{n}(\%)$ & $\chi^{2}$ & $p$ \\
\hline Video condition $(\mathrm{N}=670)$ & & 0.02 & .99 \\
\hline Low educational level & $47(20.9)$ & & \\
Middle educational level & $51(20.6)$ & & .02 \\
High educational level & $42(21.2)$ & & \\
\hline Text condition $(\mathrm{N}=708)$ & & & \\
\hline Low educational level & $33(14.3)$ & & \\
Middle educational level & $41(16.1)$ & & \\
High educational level & $53(23.9)$ & & \\
\hline
\end{tabular}

Note: $p$-values $<.05$ are marked bold.

Table 5b Abstinence rates per educational level, stratified by adherence or non-adherence towards the intervention separately for the VCT and TCT conditions

\begin{tabular}{|c|c|c|c|}
\hline & Abstinent $\mathrm{n}(\%)$ & $\chi^{2}$ & $p$ \\
\hline \multicolumn{4}{|l|}{ Video condition $(N=670)$} \\
\hline $\begin{array}{l}\text { Low educational level } \\
\qquad \begin{array}{l}\text { Adherence }=0(n=128) \\
\text { Adherence }>1(n=97)\end{array}\end{array}$ & $\begin{array}{l}17(13.3) \\
30(30.9)\end{array}$ & 10.40 & $<.001$ \\
\hline $\begin{array}{l}\text { Middle educational level } \\
\text { Adherence }=0(n=135) \\
\text { Adherence }>1(n=112)\end{array}$ & $\begin{array}{l}15(11.1) \\
36(32.1)\end{array}$ & 16.53 & $<.000$ \\
\hline $\begin{array}{l}\text { High educational level } \\
\qquad \begin{array}{l}\text { Adherence }=0(n=113) \\
\text { Adherence }>1(n=85)\end{array}\end{array}$ & $\begin{array}{l}18(15.9) \\
24(28.2)\end{array}$ & 29.93 & .04 \\
\hline \multicolumn{4}{|l|}{ Text condition $(\mathrm{N}=708)$} \\
\hline $\begin{array}{l}\text { Low educational level } \\
\qquad \begin{array}{l}\text { Adherence }=0(n=218) \\
\text { Adherence }>1(n=238)\end{array}\end{array}$ & $\begin{array}{l}8(8.9) \\
25(17.7)\end{array}$ & 3.51 & .06 \\
\hline $\begin{array}{l}\text { Middle educational level } \\
\text { Adherence }=0(n=242) \\
\text { Adherence }>1(n=260)\end{array}$ & $\begin{array}{l}8(7.5) \\
33(22.3)\end{array}$ & 10.12 & $<.001$ \\
\hline $\begin{array}{l}\text { High educational level } \\
\qquad \begin{array}{l}\text { Adherence }=0(n=177) \\
\text { Adherence }>1(n=243)\end{array}\end{array}$ & $\begin{array}{l}10(15.6) \\
43(27.2)\end{array}$ & 3.37 & .07 \\
\hline
\end{tabular}

Note: $p$-values $<.05$ are marked bold.

Abstinence rates are presented separately for the two experimental conditions and are stratified by educational level. The results revealed significantly higher abstinence rates among those who adhered to the VCT condition across all educational groups. Additionally, in the TCT 
condition abstinence rates were significantly higher among smokers with a middle educational level who adhered to minimally one further session.

\section{Differences in appreciation of the program}

Table 6 shows the program evaluation of sample 2. To test possible interaction effects between the conditions (VCT vs. TCT) and educational level on process evaluation items, ANOVAs were conducted. There was no significant interaction between condition and educational level with respect to attention $(F(1,327)=0.17, p=.84)$, comprehension $F(1,327)=1.59, p=.21)$, adaptation $(F(1,327)=1.24, p=.29)$, appreciation $(F(1,326)=0.07, p=.93)$ or processing $(F$ $(1,318)=0.01, p=.99)$ of the feedback messages. As shown in Table 6 , the video feedback messages seem to be slightly better evaluated than the text-based messages in terms of appreciation and processing, but the differences did not reach significance.

Table 6 Means and standard deviations (SD) for evaluation of different aspects of the intervention program at sixmonth follow-up

\begin{tabular}{lllll}
\hline Evaluation items & $\begin{array}{l}\text { Overall sample } \\
(\mathrm{n}=333)\end{array}$ & $\begin{array}{l}\text { Video } \\
(\mathrm{n}=142)\end{array}$ & $\begin{array}{l}\text { Text } \\
(\mathrm{n}=191)\end{array}$ & $p$ \\
\hline $\begin{array}{l}\text { Feedback was attractive } \\
\text { (attendance); M (SD) }\end{array}$ & $3.40(1.04)$ & $3.49(1.07)$ & $3.37(1.03)$ & .18 \\
$\begin{array}{l}\text { Feedback was } \\
\text { understandable } \\
\text { (comprehensibility); M (SD) }\end{array}$ & $3.63(0.70)$ & $3.69(0.70)$ & $3.58(0.70)$ & .15 \\
$\begin{array}{l}\text { Feedback fit to own } \\
\text { situation; M (SD) }\end{array}$ & $3.31(0.74)$ & $3.35(0.78)$ & $3.28(0.71)$ & .40 \\
$\begin{array}{l}\text { Feedback was useful } \\
\text { (appreciation); M (SD) }\end{array}$ & $3.54(0.96)$ & $3.64(1.02)$ & $3.45(0.90)$ & .07 \\
$\begin{array}{l}\text { Feedback helped to make } \\
\text { quit attempt (processing); M (SD) } \\
\begin{array}{l}\text { Overall grade of feedback } \\
\text { (from 1-10); M (SD) }\end{array}\end{array}$ & $3.27(0.86)$ & $3.37(0.90)$ & $3.20(0.82)$ & .06 \\
\hline
\end{tabular}

\section{Discussion}

\section{Principal findings}

The aim of this study was to evaluate the effects and appreciation of two multiple computertailored smoking cessation interventions (video- vs. text-based messages) delivered via the Internet, regarding six-month smoking abstinence among different educational groups. To our knowledge, this study is one of the first studies to test the effects of mode of delivery in the context of smoking cessation. Low levels of adherence may lead to an underestimation of the effects, therefore, the effectiveness of the two computer-tailored interventions was assessed by analysing two samples. The first sample included all randomly assigned respondents who filled in the baseline questionnaire and followed the first session of the intervention whereas 
the second sample was a subsample of sample 1 including only respondents (in the experimental conditions) who at least adhered to one further session of the intervention.

Our study revealed several important findings. In contrast to our expectations, the results of all analyses revealed no significant differences in quit rates between smokers with low and high educational levels in the two experimental conditions (video- vs. text-based messages). However, in both samples, the video-based multiple computer-tailored smoking cessation intervention was effective in increasing seven-day point prevalence abstinence. The text-based multiple computer-tailored smoking cessation intervention, however, was only significantly effective in increasing seven-day point prevalence abstinence in people who adhered at least one further session (after baseline and session 1). The video-based condition was also more effective compared to the text-based condition, regarding seven-day point prevalence abstinence in sample 2 .

Moreover, with regard to prolonged abstinence our study revealed a differential effect of the intervention between people with a low or high readiness to quit, consistent with our second expectation. In sample 1, the video-based computer-tailored intervention appeared to be especially successful in increasing prolonged abstinence rates among smokers with a lower readiness to quit (four to six months) whereas in sample 2 , the video-based computer-tailored intervention was also effective among smokers willing to quit within one month. The multiple imputation and the complete case analyses yielded comparable results, a finding that may be attributed to the fact that rates of missing data were not extremely high at six-month followup (on average 30\%).

Consistent with previous findings (Campbell et al., 1999, Houts et al., 2006) in other behavioural domains, the results of our study indicate that tailored video-based messages might be more effective in supporting smokers to quit smoking regarding seven-day point prevalence abstinence compared to tailored text-based messages. Using video-based messages was equally effective in smokers of all educational levels. This is in line with past research which found that Internet users appreciate the concept of tailored video interventions over text-based interventions (Vandelanotte and Mummery, 2011). Contrary to our first hypothesis, the video-based computer-tailored intervention was not more effective in less educated groups than in highly educated groups. The delivery of information on the Internet is rapidly changing with the proportion of video content increasing (Purcell, 2010); therefore it is possible that there is a general preference for receiving tailored information in a video format. In line with this, our program evaluation also revealed a slightly better evaluation of the tailored video messages. Again, no differences were recognized between lower and higher educated respondents.

Our study revealed another interesting effect of the video-tailored intervention for people with a lower readiness to quit smoking. With different routings available in our smoking cessation intervention, we expected that both interventions would be effective for people with 
a lower motivation to quit at baseline. Partially consistent with our second hypothesis, the results revealed that only the video tailored intervention appeared to be successful in smokers with a lower readiness to quit. The availability of different intervention routings provided these less motivated smokers the possibility to reflect on their smoking behaviour and their potential quit attempt; these less motivated smokers may have benefited from this option in the videobased condition.

Consistent with our expectation, our study showed that abstinence rates were higher overall when respondents adhered to at least one further intervention element. In sample 2 , adherence can be regarded as a determinant of the efficacy of the program. These findings are in line with different previous research, which also found that the efficacy of a program increased when people adhered to the intervention (Borland et al., 2004, Elfeddali et al., 2012a).

\section{Strengths and limitations}

To our knowledge, this is the first study to assess the effectiveness of a web-based tailored video and text intervention aiming to promote smoking cessation in groups with varying levels of education and varying levels of readiness to quit. A strength of the study is that two different sensitivity analyses were performed to test the robustness of our results. However, our study is also subject to several limitations. First, a misreport may have occurred when respondents were asked to indicate their smoking status at the six-month follow-up measurement. For financial reasons, we were not able to biochemically validate respondents' self-assessed smoking status. Although future web-based intervention studies might be recommended to verify smoking status through the use of biochemical cotinine test as part of a more detailed follow-up assessment, it is also argued that this might be irrelevant, e.g. if anonymity has been guaranteed (Velicer et al., 1992). It may be that web-based interventions are attractive because they enable people to participate anonymously. This topic requires further elaboration in future studies. Second, we assessed smoking status after six months; however, it might be valuable to replicate these findings and investigate whether these results persist over a longer follow-up period. Lastly, during our intervention, respondents were not able to choose a quit date within a week of baseline. Although, there is value in taking advantage of current motivational readiness and not delaying an attempt, this could be seen as a weakness of our intervention (West and Sohal, 2006).

Despite these limitations, the present study provides evidence that video-based messages are successful in stimulating quitting behaviour. As past research has already indicated that Internet users prefer to receive content in the form of video-based messages (Vandelanotte and Mummery, 2011) and our results confirmed this, the use of audiovisual content might increase the appeal of future health interventions and smoking cessation interventions in particular. 


\section{Conclusion}

The current study provides important new evidence for the effectiveness of a video-based multiple computer-tailored smoking cessation intervention. The results suggest that a videobased computer-tailored intervention with personalised feedback adapted to the smokers' motivation to quit might be effective in increasing abstinence rates for smokers with different educational levels. The results support the feasibility of using video messaging to affect smoking behaviour. We measured smoking abstinence after six months; more research is needed to examine whether these results persist over longer follow-up periods. 



\section{CHAPTER 6}

Effectiveness of a video- and text-based computer-tailored smoking cessation intervention among LSES and HSES smokers

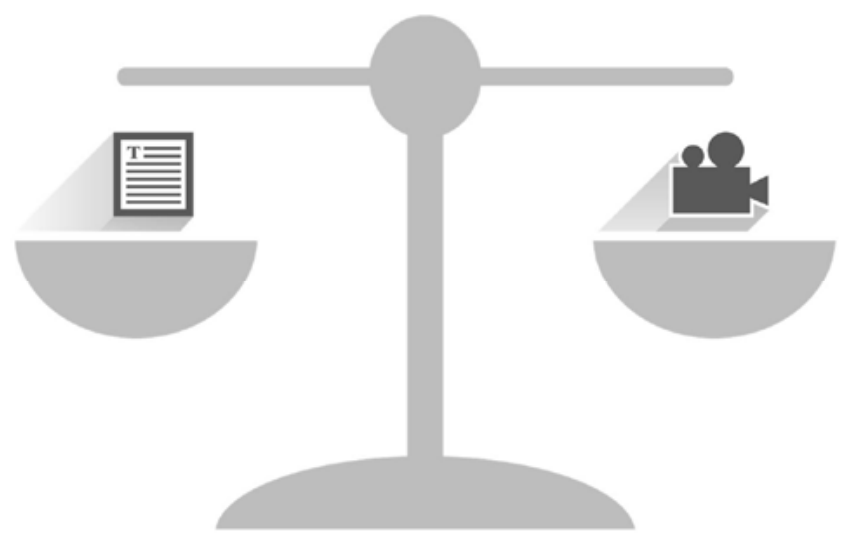

Submitted as:

Stanczyk, N. E., de Vries, H., Candel, M. J. J. M., Muris, J. W. M. \& Bolman, C. Effectiveness of a video- and text-based computer-tailored smoking cessation intervention among LSES and HSES smokers. 


\begin{abstract}
Background: In western countries smoking prevalence rates are high among unmotivated people and people with a lower socioeconomic status (LSES). Multiple computer tailoring and the use of visual aids may improve such interventions and increase cessation in LSES smokers. This study assessed the 12-month effectiveness of a video- and text-based computer-tailored intervention, and potential differential effects for subgroups varying in socioeconomic status (SES) and motivation to quit.
\end{abstract}

Methods: A randomized controlled trial was used in which smokers were allocated to the videobased condition ( $n=670)$, the text-based condition $(n=708)$ or the control condition (brief generic text advice) ( $n=721$ ). After 12 months, self-reported prolonged abstinence was investigated and biochemically verified in respondents who indicated they had quit smoking. Multiple imputation analyses were used as primary analyses, whereas complete case and a negative scenario (respondents lost to follow-up were considered as smokers) were conducted as secondary analyses.

Results: Primary analyses revealed that the video-based computer-tailored intervention was more effective on prolonged abstinence compared to the control condition $(\mathrm{OR}=1.90, p=$ .005) and the text-based condition (OR = 1.71, $p=.01)$. No differential effects were found for people with different SES levels and different motivational levels. Results were confirmed in the complete cases and negative scenario analyses.

Conclusion: The video-based computer-tailored intervention is effective to help smokers in achieving long-term smoking abstinence.

Key words: smoking cessation, multiple computer tailoring, indicators of socioeconomic status, income, occupational status, educational level, text-based messages, video-based messages 


\section{Introduction}

The problem of tobacco use is relevant to people with a lower socioeconomic status (LSES) (Jha et al., 2006, Kotz and West, 2009). In western countries, smoking prevalence rates are higher among LSES groups (Hiscock et al., 2011, Nagelhout et al., 2012). Studies reveal higher levels of addiction among this group, as well as lower levels of motivation and more relapse to smoking (Hiscock et al., 2011, Kotz and West, 2009).

Web-based computer-tailored smoking cessation interventions can be successful in increasing smoking abstinence rates and are (cost) effective (Smit et al., 2013, Strecher et al., 2008a, Te Poel et al., 2009). Computer-tailored messages are successful in keeping an individual's attention, resulting in better processing of the information (Hawkins et al., 2008, Ruiter et al., 2006). Although computer-tailored smoking cessation interventions have shown to be effective, research indicates that they may have difficulties to attract LSES groups (Brouwer et al., 2010). Furthermore, adherence to these interventions is especially low among unmotivated and LSES smokers (Moser et al., 2000, Strecher et al., 2008b). It is therefore, important to investigate how these interventions can be improved to attract different SES groups.

One possibility might be not to focus solely on smokers who are highly motivated to quit; most smoking cessation interventions target highly motivated smokers (Echer and Barreto, 2008). LSES smokers are often less motivated to quit, and as such, they may benefit from programs stimulating them to reflect on their smoking behaviour first, before directly advising them to quit. Hence, computer-tailored smoking cessation interventions could be more appealing for LSES smokers if they provide tailored feedback for different levels of motivation.

Another strategy is to use video-delivered information. Video information may be more effective in stimulating comprehension among LSES groups (Wilson et al., 2010). Consequently, the use of videos may improve the appeal of computer-tailored e-health smoking cessation interventions (Frenn et al., 2005).

This study assessed the effectiveness of two computer-tailored smoking cessation interventions after 12 months: 1) a text-based multiple computer-tailored intervention providing tailored feedback via text-based messages and 2) a video-based multiple computertailored smoking cessation intervention providing tailored feedback via video-based messages. Smokers could follow different routings that were tailored to their motivation/readiness to quit. By this latter approach, we expected smokers with a lower motivation to quit to be equally effective in their quit attempt as higher motivated smokers. Furthermore, we hypothesised that video computer tailoring (VCT) would be more successful in smokers with a LSES, whereas text computer tailoring (TCT) was expected to be more effective in smokers with a higher socioeconomic status (HSES) (Stanczyk et al., 2011). 
Measuring the impact of interventions among SES groups presupposes the existence of a clear operationalisation of SES. Educational level is a key indicator for monitoring inequalities in smoking, reflecting knowledge and skills which are necessary for successful quitting (Laaksonen et al., 2005). Occupational status, on the other hand, is associated with workrelated factors such as job stress and social influence, factors that often hinder successful quitting. Income has been used as an indicator of material deprivation and is often associated with persistent smoking problems (Laaksonen et al., 2005, Schaap and Kunst, 2009). To investigate possible differential intervention effects for SES groups, we took several socioeconomic measures (educational level and occupational status) into account.

\section{Methods}

The Medical Research Ethics Committee (MREC) of Atrium Medical Centre Heerlen judged that the study was not obliged to MREC approval, as respondents did not have to engage in medical acts. The study fulfilled the American Psychological Association (APA) ethical principles and was registered in the Dutch Trial Register (NTR3102).

\section{Recruitment}

A sample of 150 general practitioners (GP) was asked to refer smokers to the intervention website. Respondents were also recruited by advertising campaigns in newspapers and websites of health funds. We recruited respondents by social network websites such as Facebook. Advertisings directly referred smokers to the intervention website.

\section{Target population and inclusion criteria}

Respondents (i.e. daily smokers of 18 years or older, motivated to quit within six months) were recruited from December 2010 until June 2012 to take part in the study.

\section{Design and procedure}

The study concerned a randomised controlled trial. Smokers could sign in via the intervention website (http://www.steunbijstoppen.nl). Respondents were randomised to one of the two experimental conditions (TCT vs. VCT) or the control condition (CC). After giving online informed consent, respondents had to complete the baseline measurement. Tailored to the quit motivation, respondents received feedback over a period of three months (see Figure 1). Respondents in the TCT condition received tailored feedback via text-based messages. In the VCT condition, adults in video messages gave the same messages. Respondents in the CC received only one general text advice. Six and 12 months after baseline, respondents were invited to complete the follow-up measurements. 


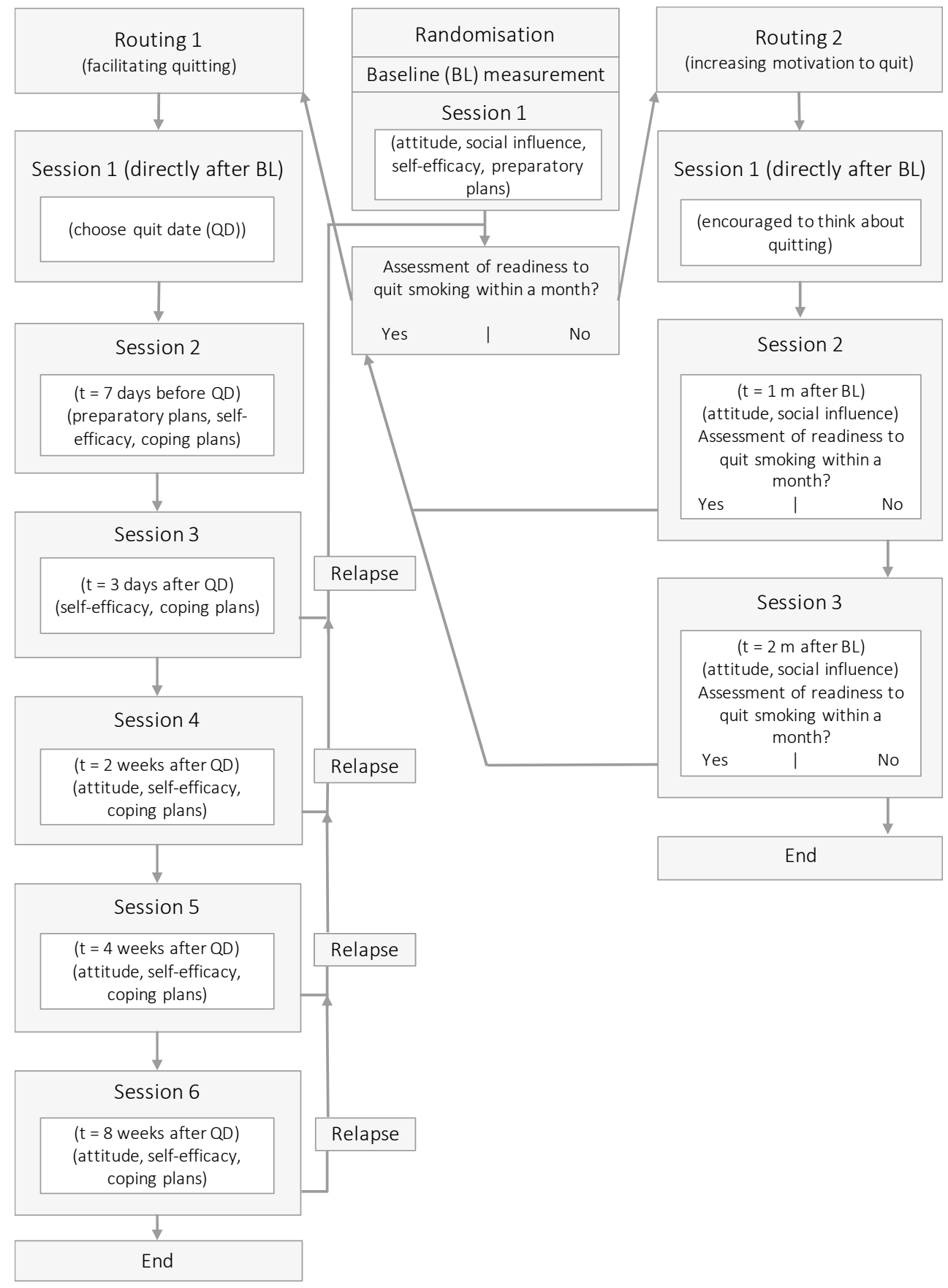

Figure 1 Two routings of a video- and text-based computer-tailored intervention 


\section{Intervention}

The two web-based multiple computer-tailored smoking cessation interventions (TCT vs. VCT) were based on two earlier computer-tailored smoking cessation interventions (Smit et al., 2012, Te Poel et al., 2009). Respondents in the two computer-tailored interventions received personalised feedback on their smoking behaviour, attitude, perceived social influence, selfefficacy and how to prepare to quit. Respondents received feedback during several feedback sessions, either in routing 1 or 2 . Respondents who set their quit date within a month were directed to routing 1 , aiding smokers to translate their intention to quit smoking into action. Respondents not ready to quit within one month were directed to routing 2, aimed at increasing smoker's motivation to quit. A detailed description of the intervention components is provided elsewhere (Stanczyk et al., 2011).

\section{Measurements}

\section{Baseline measurement}

Demographic variables age, gender $(0=$ male; $1=$ female), and nationality $(0=$ other nationality; 1 = Dutch nationality) were assessed. Socioeconomic status was measured by educational level, occupational status and income.

1) Educational level was measured by one item asking respondents which educational level they had completed (Mudde et al., 2006). Education was divided into three groups: $1=$ low (primary, basic vocational, lower education or no education); 2 = intermediate (higher general secondary education, preparatory academic education, medium vocational education); and 3 = high (higher vocational education or university level) (CBS, 2012).

2) Occupational status was measured by two items (Mudde et al., 2006). Respondents were first asked to indicate their working situation ( 1 = freelance; $2=$ employed; $3=$ working for the public sector; 4 = incapable of work; $5=$ unemployed; 6 = retired; 7 = student; $8=$ housewife/houseman). Respondents were then asked to specify their occupational level: $1=$ gardener/farmer; 2 = own company; 3 = free occupation (e.g. lawyer, physician); 4 = higher occupational level (e.g. manager, therapist); $5=$ middle occupational level (e.g. lab assistant, nurse); 6 = lower occupational level (e.g. sales assistant).

3) Income was measured by one item asking respondents to indicate their household income, excluding pre-taxes, resulting in a 10-point scale from $€ 0$ to $\geq € 3,400$ (CBS, 2011b).

4.) Housing tenure was measured by one item asking respondents whether they rent or own their housing ( 1 = rent; 2 = own). Housing tenure was combined with income and educational level to achieve an overall 'wealth' measure (Mudde et al., 2006). As income had a high rate of missing values (14.3\%), it was decided to exclude the 'wealth' measure in the operationalisation of SES. Furthermore, occupational status and educational level was combined into one measure (CBS, 2011b). 
Addiction level was measured by six items using the Fagerström Test for Nicotine Dependence (FTND) (0 = not addicted; 10 = highly addicted) (Heatherton et al., 1991, Fagerström, 2012). The items were summed into an index.

Readiness to quit smoking was assessed by one item asking respondents whether and when they intended to quit smoking, resulting in three categories ( $1=$ yes, within four to six months; 2 =yes, within one to three months; 3 =yes, within the following month) (De Vries et al., 2008).

Depressive symptoms were measured by the abbreviated CES-D, resulting in a fourpoint scale ( 1 = rarely or never to $4=$ most or all times) (Cole et al., 2004); a sum score was included in the analyses (Cronbach's alpha $=.85$ ).

Smoking habit was assessed using an abbreviated version of Verplanken and Orbell's Self-reported Habit Index of six items (e.g. Smoking is something which I do automatically), resulting in a five-point scale ( $1=$ I totally disagree to $5=$ I totally agree) (Verplanken and Orbell, 2003).

Occurrence of smoking-related diseases was measured by four questions on a dichotomous scale ('Do you suffer from chronic respiratory disease, cancer, diabetes or cardiovascular disease?'; 0 = no or 1 = yes).

Attitude was measured by three items assessing the pros and cons of quitting, resulting in a five-point scale ( $1=\mid$ totally disagree to $5=\mid$ totally agree). A mean scale score was included in the analyses (Cronbach's alpha $=.52$ ).

Social influence was measured by two scales. Social modelling was assessed by two items that measured whether other people in the environment smoked (respectively partners ( 1 = no; 2 = yes; 9 = not applicable) and social environment (family or friends; 1 = none; 2 = a minority; 3 =half; 4 = a majority; 5 = all; 9 = not applicable)). A number of respondents ( $n=552$ ) selected 'not applicable' when asked whether their partner smoked, 80 selected this choice when asked whether someone in their social environment smoked. Social support was measured by two items that asked whether smokers received social support (respectively partners and social environment) in favour of quitting on a four-point scale ( $1=$ no; 2 = yes, a bit; 3 = yes, moderate; 4 = yes, a lot; 9 = not applicable). Seven hundred and eighty-seven respondents selected 'not applicable' when asked whether they received support from their partner, 229 selected 'not applicable' when asked whether they received support from their social environment. 'Not applicable' was recoded into the lowest value ( $1=$ no support) for the social influence measure; items were summed into an index.

Preparatory plans were assessed by three items that measured whether respondents planned to execute different preparatory plans for their quit attempt (e.g. removing ashtrays) on a five-point scale ( 1 = surely not to 5 = surely yes).

Coping plans were assessed by four items that measured whether respondents had made specific plans to prevent relapse in difficult situations ( $0=$ no; $1=$ yes). 
Self-efficacy was measured by three items asking respondents whether they would be able to refrain from smoking in these difficult situations, resulting in a five-point scale ( $1=$ definitely not to $5=$ definitely yes). A mean scale score was included in the analyses (Cronbach's alpha $=.62)$.

\section{Follow-up measurement}

At 12-month follow-up, prolonged abstinence was the main outcome and was measured by one item asking respondents whether they refrained from smoking (including a grace period where they could have smoked one to five cigarettes within two weeks) since their last quit attempt ( 0 = no; 1 = yes; self-report) (Hughes et al., 2004, Mudde et al., 2006). Those who reported that they had quit less than nine months before the follow-up were regarded as smokers (Hughes et al., 2004). For secondary analyses, seven-day point prevalence abstinence was assessed by one item asking respondents whether they had refrained from smoking during the last seven days ( $0=$ no; 1 = yes) (Hughes et al., 2004, Mudde et al., 2006).

\section{Biochemical validation}

Respondents who indicated that they had quit smoking after 12 months ( $n=167$ ) follow-up were invited to biochemically validate their self-reported smoking status. NicAlert ${ }^{\circledR}$ test strips were used to measure cotinine in saliva. A cut-off point of $\geq 2$ for saliva indicated that respondents still smoked tobacco (Marrone et al., 2011). Sixty-two respondents (37\%) completed the test and sent it back to the research team. In 95.2\% ( $n=59)$ the cotinineassessment verified the non-smoking status. In $4.8 \%(n=3)$ cotinine was detected. Smoking status of these respondents was changed to 'smoker.'

\section{Statistical analysis}

Descriptive analyses were performed to test for differences between the three conditions. To detect possible drop-out at follow-up, logistic regression was used, including baseline factors and group assignment as predictors. All significant baseline differences and predictors of dropout were included in all effect analyses. Next, logistic regression analyses were performed to investigate the 12-month effectiveness of the intervention on smoking abstinence. Potential confounders, including demographic variables, variables with an expected independent influence on smoking cessation, baseline differences, drop-out predictors and different interaction terms (SES ('educational level' and 'occupational status with educational level') by condition and readiness to quit smoking by condition) were included as predictor variables. A top-down procedure was used, first removing non-significant interaction terms. In the effect analyses, we used a multiple imputation dataset (Schafer, 1999). Additionally, we tested the outcomes in a complete-case scenario (including only respondents who successfully completed 
the follow-up measurement) and a negative scenario (respondents missing at follow-up were regarded as smokers).

Data were analysed using SPSS 19.0 (SPSS, Inc., Chicago, IL. USA).

\section{Results}

\section{Sample characteristics}

Figure 2 presents the flow of respondents ( $N=2,099$ respondents). Respondents included in the analyses had a mean age of 45.7 years (SD 12.8), 1,278 (60.9\%) of them were female and 705 (33.6\%) had a low level of education. Additionally, respondents smoked on average about 19 (SD 8.6) cigarettes per day. Table 1 shows the baseline differences between the three conditions.

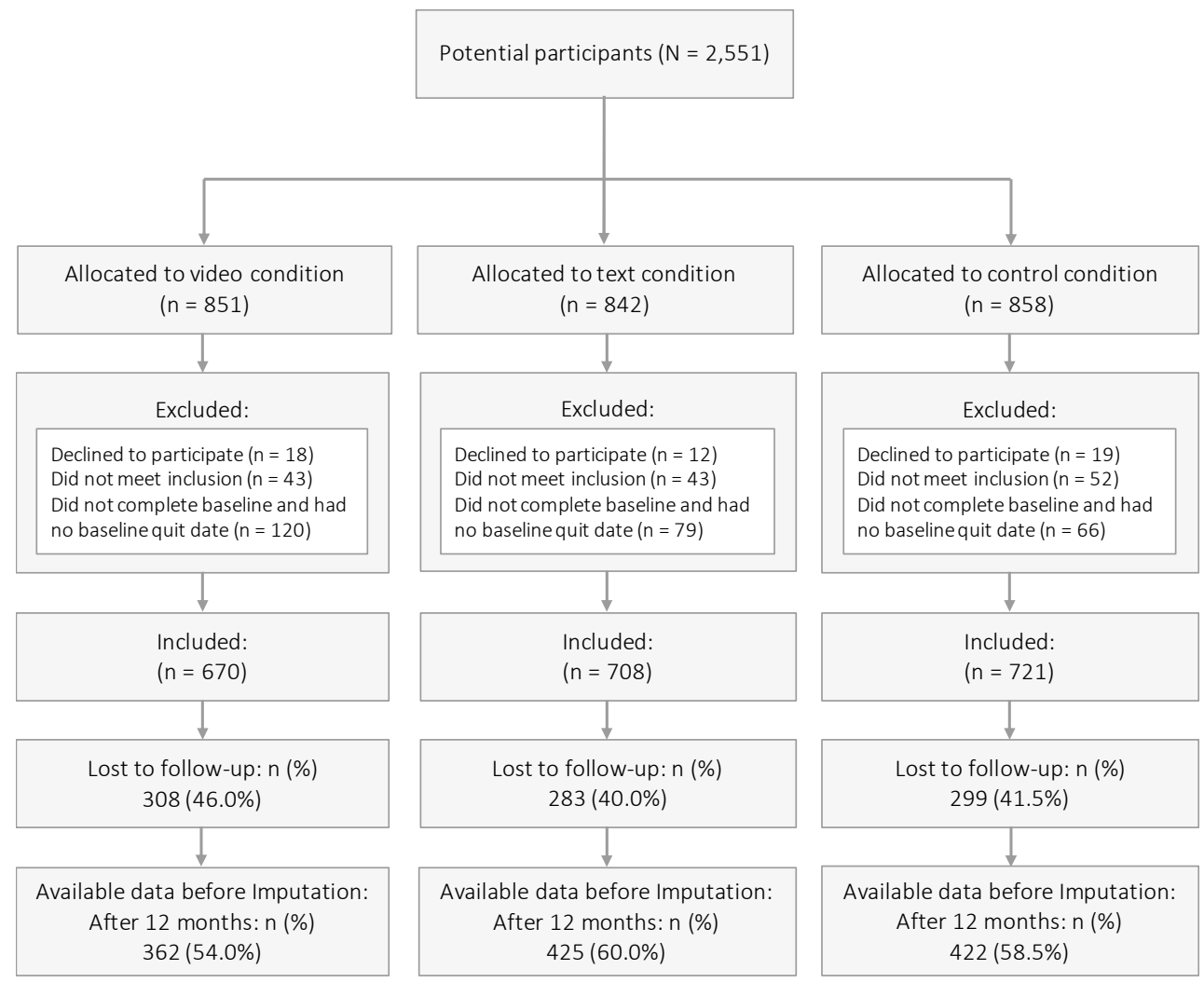

Figure 2 Flowchart of respondent's enrolment and inclusion 


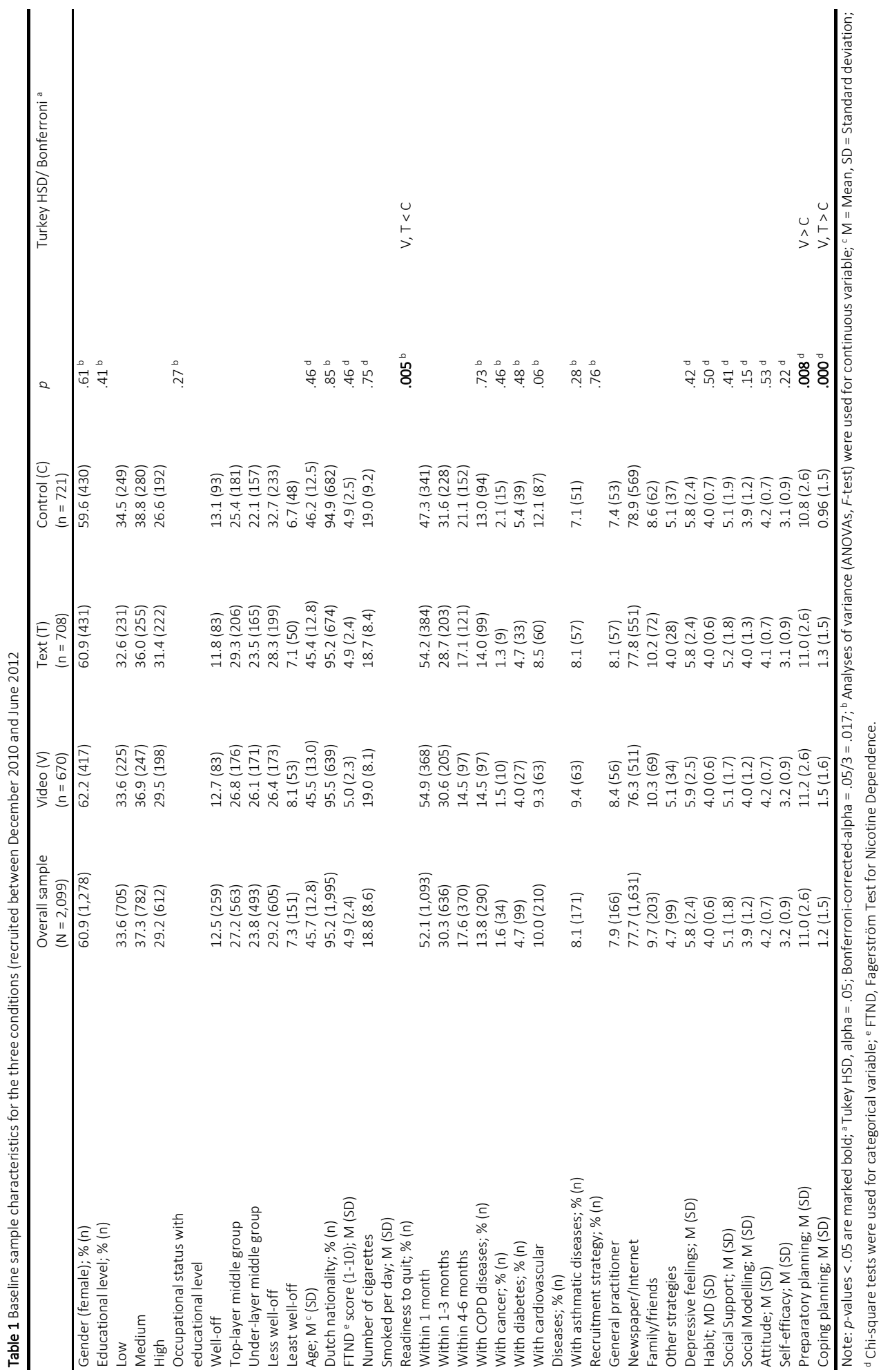




\section{Drop-out analysis}

During this study, 362 out of 670 (54.0\%) respondents were followed-up after 12 months in the VCT condition, versus 425 (60.0\%) out of 708 in the TCT condition and 422 (58.5\%) out of 721 in the CC. Retention after 12 months was significantly higher when respondents were older $(\mathrm{OR}=1.02,95 \% \mathrm{Cl} 1.01-1.03, p=.001)$, had a Dutch nationality $(\mathrm{OR}=1.63,95 \% \mathrm{Cl} 1.01-2.61$, $p=.04)$ and when they had fewer smokers in their environment $(\mathrm{OR}=0.91,95 \% \mathrm{Cl}$ 0.84-0.99, $p=.03)$.

\section{Quit rates after 12-month follow-up}

Table $2 a$ presents the quit rates for prolonged abstinence and seven-day point prevalence abstinence. In the VCT condition, a total of 66 (9.0\%) respondents reported not having smoked since their last quit date (prolonged abstinence), in the TCT condition 52 (7.3\%) respondents reported not having smoked, and $46(6.4 \%)$ respondents reported this in the control condition. Table $2 \mathrm{~b}$ and $2 \mathrm{c}$ present the prolonged abstinence and seven-day point prevalence abstinence rates for the three different conditions, stratified by educational level.

\section{Spearman correlations}

The Spearman correlation between the two variables 'educational level' and 'occupational status with educational level' was 0.71. The Variance Inflation factor (VIF) was below five, indicating no problem of multicollinearity. Both variables were used in the same regression models.

\section{Effects of the intervention at 12-month follow-up}

We assessed interaction effects between condition and the different operationalisation's of SES and condition and readiness to quit smoking. None of these were significant nor influenced smoking abstinence after 12 months.

However, multiple imputation analysis showed a main effect of condition on prolonged abstinence. The VCT condition was more effective than the CC (OR $=1.90, p=.005)$ and the TCT condition (OR $=1.71, p=.01$ ) (see Table 3). Complete cases (not presented in Table) revealed comparable results. The VCT condition was significantly more effective than the CC $(\mathrm{OR}=1.67, p=.02)$ and more effective than the TCT condition ( $\mathrm{OR}=1.59, p=.03)$. Analyses were also conducted in a negative scenario (not presented in Table). The differences between the VCT and the CC were borderline significant (OR = 1.38, $p=.06)$. The two experimental conditions (VCT vs. TCT) did not differ from each other ( $\mathrm{OR}=1.38, p=.10)$. For seven-day point prevalence abstinence, we were neither able to replicate our findings with the multiple imputation analyses (see Table 3), complete case or negative scenario. 

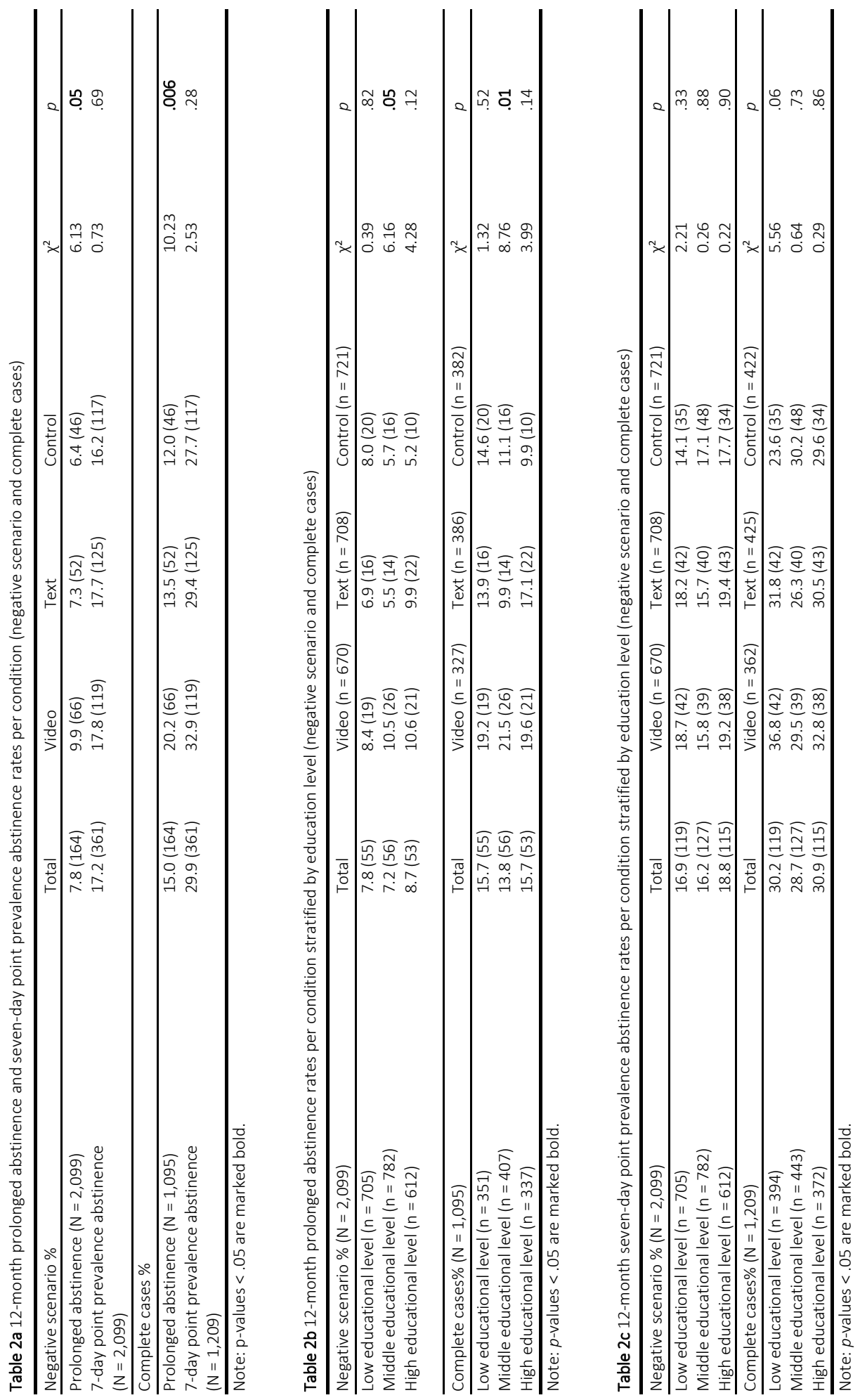


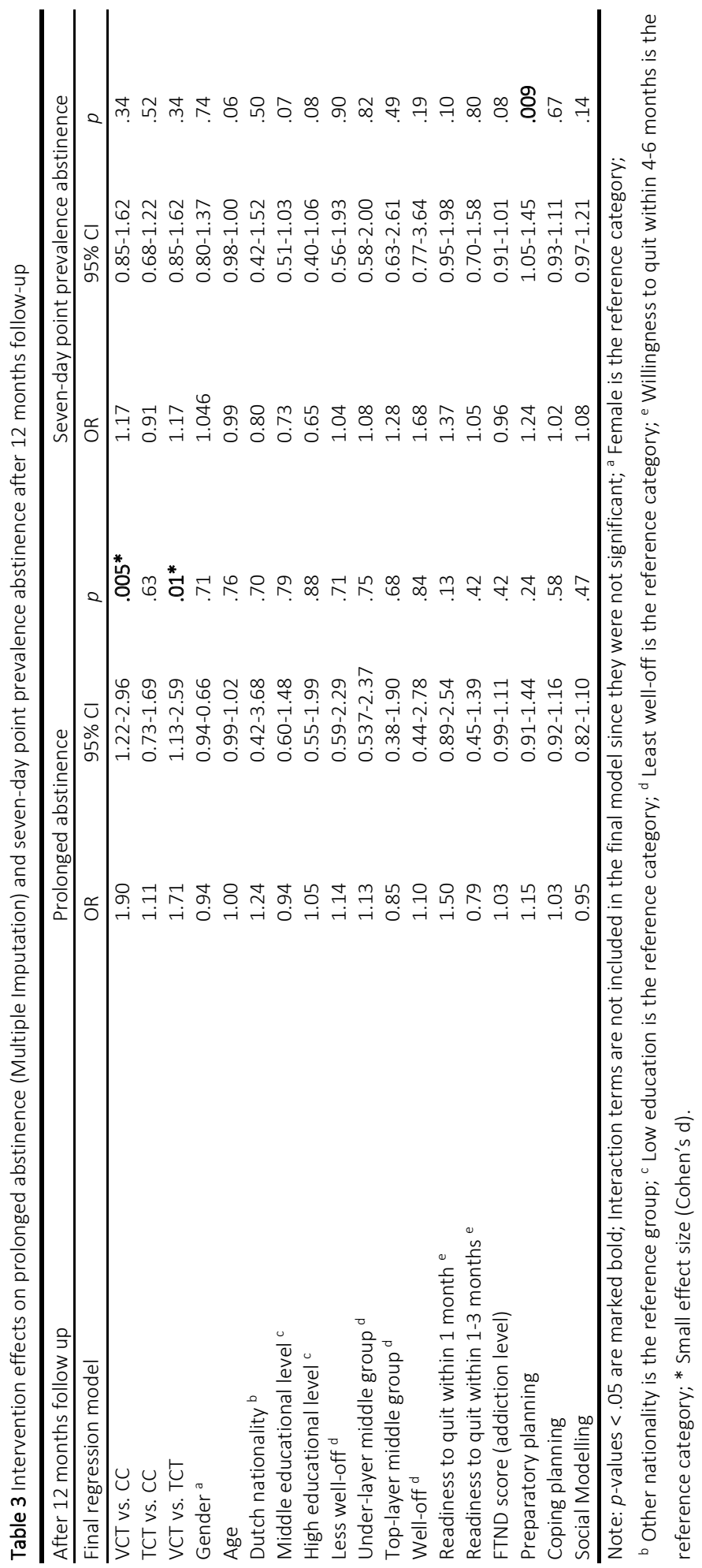




\section{Discussion}

\section{Main findings}

The study assessed the effectiveness of two computer-tailored smoking cessation interventions on smoking abstinence after 12-month follow-up. The video-based computertailored smoking cessation intervention was more effective for prolonged abstinence than the text-based intervention and the CC. No interaction was found between the condition and SES or between condition and motivation to quit smoking.

Confirming the results of earlier studies in other health-related domains (Frenn et al., 2005, Tuong et al., 2012), our study revealed that the video-based intervention is an effective strategy for increasing smoking abstinence. Although we hypothesised that LSES smokers might profit more from video computer tailoring and HSES smokers more from text computertailoring, we did not find this moderating effect of SES and abstinence rates were similar for LSES and HSES smokers. As information delivery via the Internet increasingly uses videos (Vandelanotte and Mummery, 2011) and our video-based intervention was slightly better evaluated by respondents during our six-month process evaluation - possibly caused by increased use of videos on the Internet, an explanation is that adults in general have started to prefer video information over text-based information, leading to similar effects for our SES groups and motivational groups. The latter finding supported our hypothesis that the use of a tailored approach using different routings for more and less motivated respondents was valuable.

Intervention effects could not be demonstrated when seven-day point prevalence abstinence was used as an outcome measure. Since seven-day point prevalence is able to include smokers who made a delayed quit attempt (not within in the intervention period itself), it might have underestimated the intervention's effectiveness.

Lastly, socioeconomic indicators did not predict smoking abstinence. A possible explanation is that the traditional measurements (such as educational level or occupational status) alone failed to reflect the complexity of SES. Since our question about income also showed to be sensitive for non-response, as in other research (Moore et al., 2000), other indicators should be also included in future research.

\section{Strengths and limitations}

The study strengths are that this was one of the first studies investigating the long-term effectiveness of a video- and text-based smoking cessation intervention, that two sensitivity analyses were performed, that we used a strong research design and had low drop-out rates comparable to similar studies (Smit et al., 2012).

Our study is subject to some limitations. First, measurement of SES has been shown to be subject to criticism and debate (Laaksonen et al., 2005). SES was indexed by respondent's 
educational level and their occupational status. Preferably, SES might have been operationalised on respondent's household income (Laaksonen et al., 2005, Schaap and Kunst, 2009). Yet, household income had high rates of missing values (14.3\%) and was therefore not included. Second, due to financial and logistic reasons, respondents themselves completed biochemical validation of self-assessed smoking status at home. It might have been better to visit the respondent to ensure that biochemical confirmation has been done correctly. A small percentage (37.1\%) of all self-reported quitters completed the test. If we assume that selfreports in respondents who did not undergo the test were not completely accurate, it is possible that smoking abstinence rates have been overestimated. Next, we did not investigate whether respondents adhered to all intervention elements, this might have influenced our effects. Lastly, effect sizes between VCT and CC and VCT and TCT were small but comparable to what was found in past research (Elfeddali et al., 2012, Smit et al., 2012).

\section{Conclusion}

This study provides evidence that a video-based computer-tailored smoking cessation intervention can be effective for achieving prolonged abstinence for both LSES and HSES smokers. Further research is needed to see how these types of interventions can be improved to increase abstinence rates in LSES smokers in particular. 



\section{CHAPTER 7}

An economic evaluation of a video- and text-based computer-tailored intervention for smoking cessation: a cost-effectiveness and cost-utility analysis of a randomized controlled trial

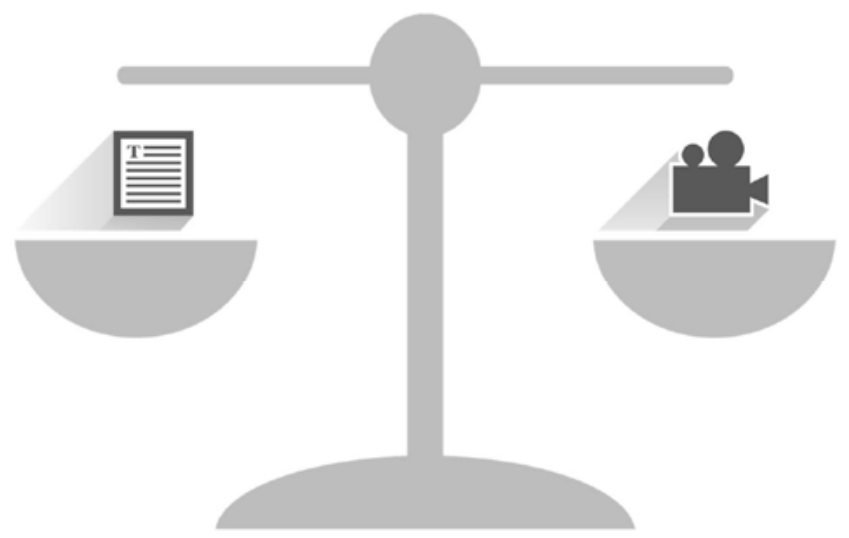

Submitted as:

Stanczyk, N. E., Smit, E. S., Schulz, D. N., de Vries, H., Bolman, C., Muris, J. W. M. \& Evers, S. M. A. A. An economic evaluation of a video- and text-based computer-tailored intervention for smoking cessation: a cost-effectiveness and cost-utility analysis of a randomized controlled trial. 


\begin{abstract}
Background: Although evidence exists for the effectiveness of web-based smoking cessation interventions, information about the cost-effectiveness of these interventions is limited.

Objective: The study investigated the cost-effectiveness and cost-utility of two web-based computer-tailored smoking cessation interventions (video- vs. text-based computer tailoring) compared to a control condition that received general text-based advice.
\end{abstract}

Methods: In a randomized controlled trial, respondents were allocated to the video-based condition ( $n=670$ ), the text-based condition $(n=708)$ or the control condition $(n=721)$. Societal costs, smoking status, and quality-adjusted life years (QALYs; EQ-5D-3L) were assessed at baseline, six-and 12-month follow-up. The incremental costs per abstinent respondent and per QALYs gained were calculated. To account for uncertainty, bootstrapping techniques and sensitivity analyses were carried out.

Results: No significant differences were found in the three conditions regarding demographics, baseline values of outcomes and societal costs over the three months prior to baseline. Analyses using prolonged abstinence as outcome measure indicated that from a willingness to pay of $€ 1,500$, the video-based intervention was likely to be the most cost-effective treatment, whereas from a willingness to pay of $€ 50,400$, the text-based intervention was likely to be the most cost-effective. With regard to cost-utilities, when quality of life was used as outcome measure, the control condition had the highest probability of being the most preferable treatment. Sensitivity analyses yielded comparable results.

Conclusion: The video-based computer-tailored smoking cessation intervention was the most cost-effective treatment for smoking abstinence after 12 months, varying the willingness to pay per abstinent respondent from $€ 0$ up to $€ 80,000$. With regard to cost-utility, the control condition seemed to be the most preferable treatment. Probably, more time will be required to assess changes in quality of life. Future studies with longer follow-up periods are needed to investigate whether cost-utility results regarding quality of life may change in the long run.

Keywords: smoking cessation, web-based computer-tailored intervention, video-based messages, text-based messages, cost-effectiveness, cost-utility, economic evaluation 


\section{Introduction}

Web-based smoking cessation interventions have large potential for public health (Civliak et al., 2010, Shahab and Mc Ewen, 2009). The enormous increase in the use of the Internet has made such interventions increasingly common all over the world (Tate et al., 2009). Different web-based computer-tailored smoking cessation interventions have already been developed and implemented to aid people to quit smoking (Civliak et al., 2010, Elfeddali et al., 2012c, Lancaster and Stead, 2005). Previous research has indicated that tailored health messages were successful in attracting smokers' attention, resulting in an enhanced processing of the health message (Dijkstra, 2005, Kreuter et al., 2003), and an increase in smoking cessation rates (Smit et al., 2012, Te Poel et al., 2009).

Even though evidence exists for the effectiveness of web-based computer-tailored smoking cessation interventions (Civliak et al., 2010, Elfeddali et al., 2012a, Smit et al., 2012), they often have difficulty attracting and holding respondents (Balmford et al., 2013, Brouwer et al., 2010, Eysenbach, 2005). Most of the web-based computer-tailored interventions consist of simple text-based messages (Civliak et al., 2010, Elfeddali et al., 2012c; Smit et al., 2012). However, since websites increasingly make use of new web-based technologies like videos or pictures, simple text-based messages may not longer be attractive enough for current Internet users. Less educated groups, in particular, often quit web-based interventions before completing all elements of the intervention (Fidler et al., 2008, Kotz and West, 2009). Since video-based messages seem to require less mental effort and may help the person to concentrate on the core elements of the message (Guimarães et al., 2000), the integration of video messages may increase the appeal and potentially the (cost) effectiveness of web-based computer-tailored smoking cessation interventions. As a result, two multiple computertailored smoking cessation interventions were developed: 1) a text-based multiple computertailored intervention, where smokers received tailored messages via text and 2 ) a video-based multiple computer-tailored smoking cessation intervention, where smokers received tailored messages via video (Stanczyk et al., 2011).

Although web-based smoking cessation interventions have already demonstrated favourable behaviour change outcomes (Civliak et al., 2010, Shahab and Mc Ewen, 2009), to date relatively little is known about whether these computer-tailored interventions are preferable in terms of their cost-effectiveness, when compared to other treatments. Given the fact that health-care budget holders have to make choices regarding the implementation of different smoking cessation programs, it is essential to know whether the societal benefits of these programs are worth the investments that have to be made to offer them (Park et al., 2013). To date, several economic evaluations of smoking cessation interventions have already been conducted (Feenstra et al., 2005, Mudde et al., 1996, Parrott et al., 1998, Song et al., 2002, Woolacott et al., 2002). The majority of these studies reported that these interventions 
were cost-effective since they were relatively low in costs accounting for the resulting gains in terms of avoided mortality and the prevention of care costs for smoking-related diseases. To our current knowledge, only one recent study in the Netherlands has investigated the costeffectiveness and cost-utility of a web-based computer-tailored smoking cessation intervention (Smit et al., 2013). This study showed that a web-based computer-tailored smoking cessation intervention seemed to be more cost-effective with regard to smoking abstinence rates assessed after 12 months than the usual smoking cessation care in the general practice setting, taking into account a willingness to pay of $€ 18,000$. However, the cost-utility analysis (calculating utility outcomes in monetary values) revealed that care as usual was the most efficient treatment (Smit et al., 2013). Even if web-based smoking cessation interventions are found to be as effective as, or less effective than traditional interventions (e.g. face-to-face counselling or care as usual), their possible lower delivery costs via the Internet may result in interventions being more cost-effective (Tate et al., 2009). As web-based interventions have the potential to contribute to reducing health-care and societal costs, economic evaluations of these interventions are required to be able to choose the most cost-effective treatment.

Given the importance of this topic, the purpose of the current study was to compare the cost-effectiveness and cost-utility of 1) a video-based multiple computer-tailored smoking cessation intervention, 2) a text-based multiple computer-tailored smoking cessation intervention, and 3) a control condition (respondents received brief general text advice about quitting) after a follow-up period of 12 months.

\section{Methods}

\section{Design and respondents}

The current economic evaluation study was embedded in a three-group randomized controlled trial $(R C T)$ that tested the effectiveness of two multiple computer-tailored smoking cessation interventions against a control condition. The study was submitted for approval to the Medical Research Ethics Committee (MREC) of the Atrium Medical Centre Heerlen, the Netherlands. The MREC decided that no MREC approval was needed for this study because respondents were not obliged to engage in a certain act. Additionally, the questionnaires of the intervention were judged not to have a deep psychological impact. Nonetheless, when submitting our study protocol for publication we were advised to register the trial and we did so shortly after the enrolment of respondents had started. We can thus confirm that all ongoing and related trials for this intervention are registered at the Dutch Trial Register (NTR3102) (Dutch Trial Register, 2013). The study was in line with the ethical codes of conduct of the American Psychological Association (APA) (American Psychological Association, 2002). Dutch smokers were included to participate in the RCT from December 2010 up to June 2012 by general practices throughout the Netherlands, several Dutch paid advertising campaigns and different health funds. 
Interested smokers could receive more information on the website and could sign up via the study website (http://www.steunbijstoppen.nl) (Steunbijstoppen.nl, 2012) by choosing their own username and password. This website was accessible on the Internet, so smokers could choose to sign up at home, at work, or anywhere else where they had access to the Internet. Smokers were randomly allocated to one of the two experimental conditions (video- vs. textbased computer tailoring) or the control condition. Randomization took place via a computer software device (OSE, 2011). After giving online informed consent, respondents were included when they were daily smokers, 18 years or older, motivated to quit smoking within the following six months and had Internet access. The flow chart of respondents is presented in Figure 1. The trial had follow-up measurements after six (between May 2011 and January 2013) and 12 months (between December 2011 and June 2013).

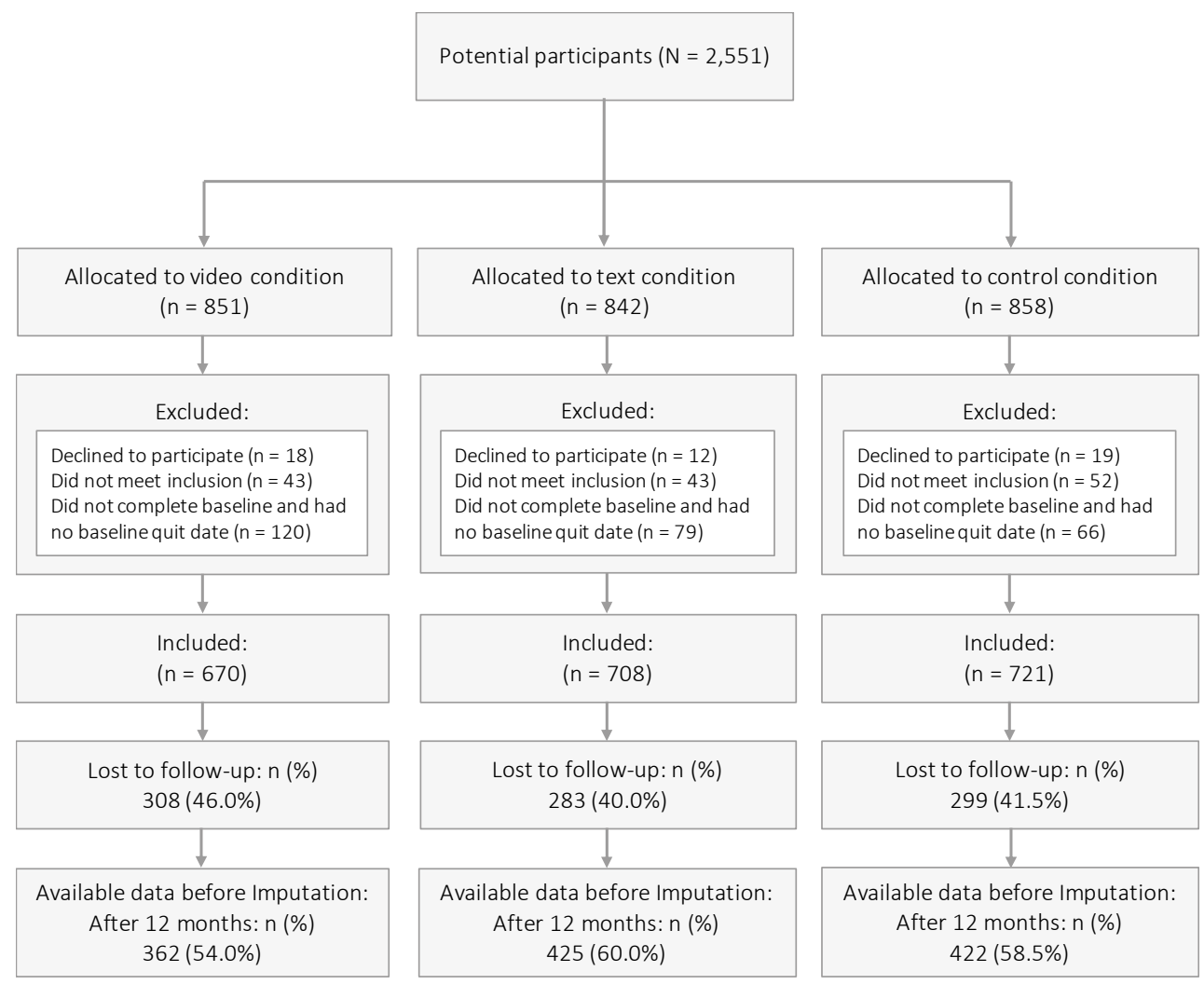

Figure 1 Flow chart of respondents 


\section{Intervention}

The two web-based multiple computer-tailored smoking cessation interventions were based on two earlier tested computer-tailored interventions that have been shown to be effective (Smit et al., 2012, Te Poel et al., 2009). The I-Change model formed the theoretical framework of the two interventions. After filling out the baseline measurement, respondents received tailored feedback on their smoking behaviour, attitude, perceived social influence, perceived self-efficacy, and on how to plan a quit date. At the end of the session, respondents were asked to set a quit date within the following month. Depending on respondents' readiness to quit smoking within the following month, they received personalised feedback during multiple computer-tailored sessions and were directed into one of two routings (see Figure 2).

\section{Routing 1}

The aim of routing 1 was to help smokers to facilitate the translation of their intention into action by providing tailored feedback to increase self-efficacy and effective action planning. Respondents ready to quit within one month were directed to routing 1 , which consisted of six different sessions: 1) at baseline, after feedback on their smoking behaviour, their attitude, social influence and self-efficacy, respondents were asked to choose a quit date; 2) one week before their quit attempt, respondents received tailored feedback on the extent to which they had planned their quit attempt (preparatory plans), on their perceived self-efficacy to deal with difficult situations and on how to cope with these situations (coping plans); 3 ) three days after their quit attempt, respondents received feedback on their perceived self-efficacy, including feedback on how to deal with difficult situations and were invited to formulate coping plans to prevent potential relapse; 4, 5 and 6) two, four and eight weeks after their quit date, respondents received tailored feedback on their perceived self-efficacy, on how to deal with difficult situations and on their attitude towards smoking and quitting smoking. During these last three sessions, respondents could choose to receive feedback on different items. This option was provided since it was expected that they would encounter different problems during their quit attempt. 


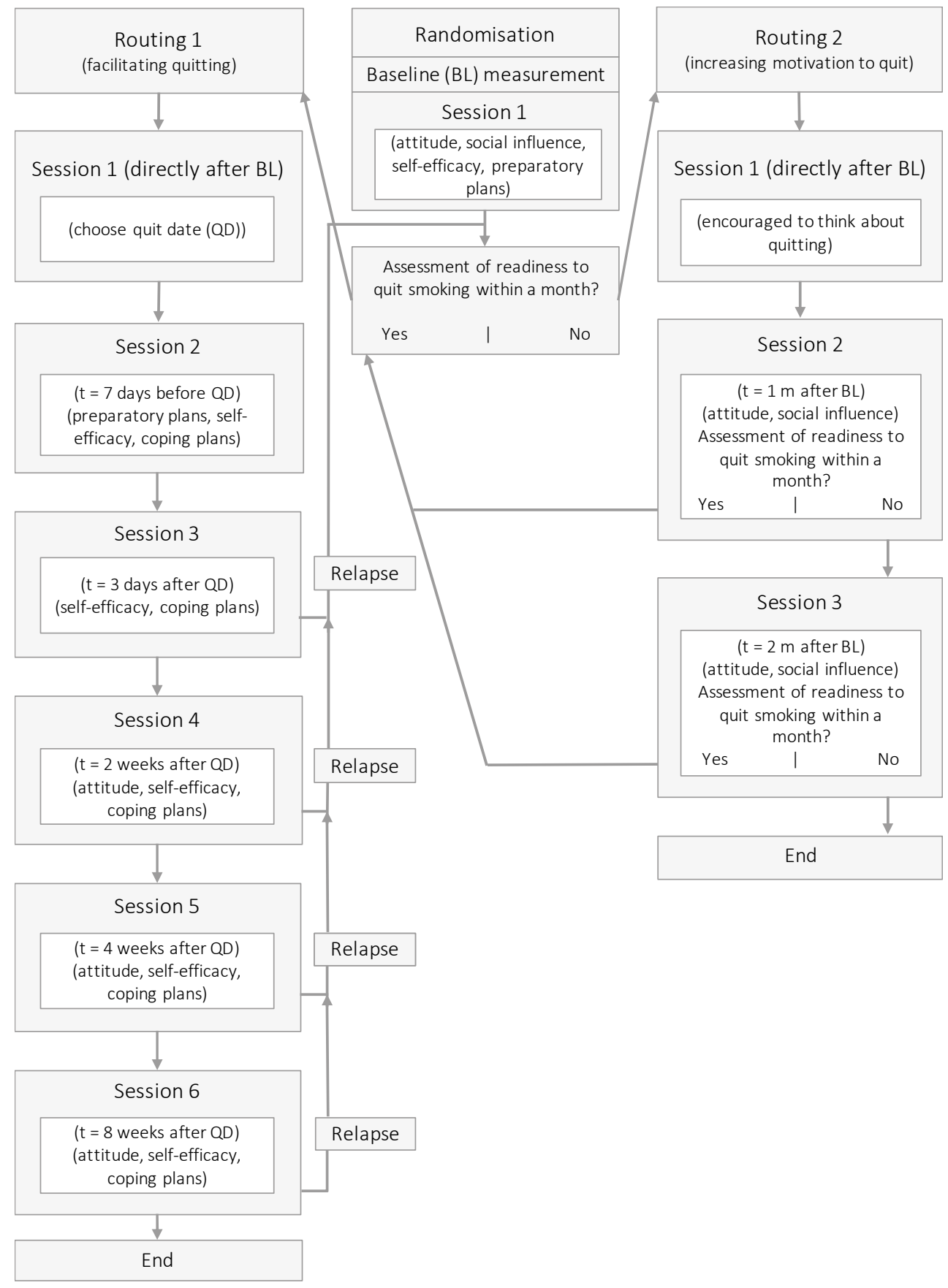

Figure 2 Intervention design 


\section{Routing 2}

The goal of routing 2 was to quit smoking and to increase motivation by increasing perceptions of the pros of quitting and how to obtain support for quitting. Respondents who were not ready to quit within one month were directed to routing 2 , which including several sessions: 1 ) directly after baseline, respondents were encouraged to use the next month to reflect on their motivation to quit smoking; 2) one month after baseline, respondents received tailored feedback on their smoking behaviour, their attitude regarding smoking and quitting smoking, their perceived social support and their readiness to quit smoking; respondents ready to quit within a month were directed to routing 1 ; 3) two months after baseline, respondents who were still not ready to quit received another tailored feedback letter, using a similar strategy as used before in session two; respondents who were ready to quit within one month were directed to routing 1 . If respondents were still not prepared to quit, they received an e-mail telling them that the program would end at this stage and that they would receive no further invitations.

After each feedback moment (in routings 1 and 2), a summary with all tailored feedback messages was sent to the respondent by e-mail. A detailed description of the study design can be found elsewhere (Stanczyk et al., 2011).

\section{Mode of delivery strategy}

In the text-based computer-tailored intervention, respondents received text-based computertailored messages (without any graphics or animations). In the video-based multiple computertailored intervention, the same tailored messages were presented as in the text-based intervention; however, the tailored messages were translated from text into videos. The tailored messages were presented by five different adults in a TV 'news program' format.

\section{Measurements}

Self-reported online questionnaires were used to assess effects and costs. Measurements were conducted at baseline, six- and 12-month follow-up. Respondents who did not complete the follow-up measurements after one and two weeks were prompted twice more by e-mail to complete the questionnaire on the intervention website. Respondents who did not react to the two reminders received another invitation by e-mail to briefly indicate their current smoking status. In this third e-mail, they were asked to fill out a shortened version of the sixand 12-month follow-up measurement, consisting of 10 important smoking-related questions, which they could send back by e-mail. Lastly, when the follow-up measurement was still not completed, respondents were contacted by a short phone call, with the same questions being asked as in the shortened questionnaire. 


\section{Baseline measurement}

The baseline measurement assessed factors related to smoking cessation and societal costs. These included demographics (age, gender, educational level), health-care-related costs and productivity costs (see below), occurrence of smoking-related diseases, and smoking-related factors such as addiction level, which was measured by the Fagerström test for cigarette dependence (FTCD) (Fagerström, 2012, Heatherton et al., 1991).

\section{Cost measures}

The present economic evaluation was conducted from a societal perspective. Intervention costs, health-care-related costs, productivity costs and respondent costs were assessed at baseline and at the follow-up measurements, using three-month retrospective questionnaires.

Intervention costs included costs that could be attributed to the delivery of the intervention, such as hosting costs for the web-based computer-tailored smoking cessation intervention. Costs for the development of the intervention and research-specific costs were excluded since these costs are non-recurring costs that will not be spent again if the intervention is implemented elsewhere.

Health-care-related costs related to general practitioners' or practice nurses' consultations or home visits, inpatient and outpatient specialist care, mental health care, alternative medicine, hospital admissions, smoking cessation aids, prescribed and over-thecounter medication, and other care (e.g. professional home care, paramedic consultations). Respondents had to indicate what type of care they had received and how often they had received it during the previous three months. The updated Dutch manual for cost analysis in health-care research was used to value the health-care-related costs of the respondents (Hakkaart-van Roijen et al., 2010). If costs for medical health care were not found in the manual, costs were searched on the Internet. Normally, the mean price of three different cost prices was used. In the case of uncertainty, the lowest cost price was taken. Furthermore, the Dutch website www.medicijnkosten.nl was used to value medication costs. The costs of medications were calculated based on the dose described by the respondent, i.e. costs per tablet, gramme or millilitre were used to calculate the total medication costs for each respondent.

Productivity costs related to absenteeism. Productivity losses for paid work were calculated according to the human capital method using a mean income valuation of the Dutch population (Hakkaart-van Roijen et al., 2010). This approach estimates the annual earnings for each year of potential lost employment and adds these earnings together. Since income differs between age and gender, productivity losses were valued differently based on respondents' age and gender (Hakkaart-van Roijen et al., 2010).

Respondent costs consisted of costs related to the time respondents spent on the computer-tailored intervention and travel costs. For the time spent on the intervention, we 
used a mean score that was needed to complete all intervention elements (without the followup measurements). A mean score of 120 minutes was used for the two computer-tailored interventions (video vs. text computer tailoring) and five minutes for the control condition. To determine the costs regarding time spent on the website, we used the human capital method using the mean valuation of the Dutch population (again, costs were valued differently based on age and gender) (Hakkaart-van Roijen et al., 2010). Travel costs were calculated based on the mean costs per kilometer and the average distance to health-care providers in the Netherlands (Hakkaart-van Roijen et al., 2010).

\section{Indexing costs}

Cost prices of the current study were from the years 2009 - 2013 and were therefore indexed to the year 2013. The consumer price indices used were 105.38 for the year 2009, 106.72 for the year 2010, 109.22 for the year 2011, 111.90 for the year 2012 and 115.00 for the year 2013 (CBS, 2013). Since the follow-up period was 12 months, discounting for differential timing was not necessary (Smit et al., 2013).

\section{Outcome measure cost-effectiveness and cost-utility}

Cost-effectiveness analysis shows the effects of alternative interventions in units that are relevant to the condition (e.g. in our case smoking abstinence) and the costs of these interventions in monetary units. It provides information about the relative efficiency of alternative interventions that aim at the same goal (e.g. smoking cessation). The benefit of our cost-effectiveness analysis is that it allows policy makers to compare the net benefit of the intervention to other smoking cessation interventions. Cost-utility analysis on the other hand is a form of cost-effectiveness analysis that aims to calculate the cost per unit of utility. A common unit of utility is quality adjusted life-years (QALY). The benefit of cost-utility analysis is that its results allow comparing outcomes of economic evaluations across diverse other health-related interventions, also (having other goals than smoking cessation, e.g. improvements in dietary behaviour or physical activity) (Gold, 1996, Siegel et al., 1996).

The primary outcome measure for the cost-effectiveness analysis (CEA) was prolonged abstinence, which was assessed by one item asking respondents whether they had refrained from smoking (including a grace period of two weeks where someone could have smoked a maximum of one to five cigarettes) since their last quit attempt ( $1=y e s ; 0=$ no). In line with the definition of prolonged abstinence, those who reported that they had quit less than nine months before the follow-up measurements were not included as quitters in the prolonged abstinence measurement (Hughes et al., 2004). A secondary outcome measure for the CEA was seven-day point prevalence abstinence, which was assessed by one item, asking respondents whether they had refrained from smoking during the last seven days ( 1 = yes; $0=$ no). 
The outcome measure for the cost-utility analysis (CUA) was quality of life, measured in terms of QALYS. In order to estimate the effects in QALYs, utility scores needed to be calculated. Utilities can be regarded as the preferences that society has for a set of healthrelated outcomes (Drummond et al., 2005). In the current study, health states were assessed by the Dutch version of the Euro-Qol (EQ-5D-3L) which is regarded as a validated measure to assess quality of life and which is often used in the Netherlands (Drummond, 2001, Drummond et al., 2005, Sculpher, 2008). The Dutch EQ-5D is a measure of self-reported health outcomes that is applicable to a wide range of health conditions. The EQ-5D-3L entails five health state dimensions: mobility, self-care, usual activity, pain/discomfort, and anxiety/depression. Each dimension was assessed by asking respondents to specify their health status, resulting in threepoint scale ( 3 = many complaints; 2 = some complaints; 1 = no complaints) (EuroQol, 1990). The health states, coming from the Euro-Qol questionnaire, were transformed into a utility score, using the Dutch tariff (Lamers et al., 2005). Utility scores could range from -0.33 (worst imaginable health status) to 1 (perfect health status). The utility scores of the three measurements (baseline, and six- and 12-month follow-up) were computed into an overall QALY score, whereby the 'area under the curve method' was used. The area under the curve is regarded as the duration of the health state, i.e. survival (on the x-axis; one year) multiplied by the quality weight for the health state ( $y$-axis; QALYs gained). In this case, the number of QALYs represents the life years gained during a one-year follow-up measurement (Drummond et al., 2005).

\section{Analyses}

\section{Baseline characteristics and dropout}

To investigate possible differences between the three conditions with regard to demographics, baseline values of the outcomes and societal costs over the previous three months, Chi-square tests and one-way analyses of variance (ANOVAs) were conducted. If Chi-square or ANOVA tests showed a $p<.05$, post-hoc pairwise comparisons were performed. Logistic regression analysis was used to explore whether there were differences between those lost to follow-up and those who remained in the study after 12 months follow-up.

\section{Imputation}

During this study, missing data were imputed by several strategies. Missing data for the costs, EQ-5D-3L questions and smoking-related variables were first replaced by mean imputation, using respondents' scores on the previous and next measurements. If we were not able to impute missing data by mean imputation, we replaced our missing data by using the last observation carried forward technique (LOCF). Unrealistic values (e.g. more than 150 meetings with a psychologist during the last three months) were replaced into the highest possible value (Hendriks et al., 2013). The same imputation method was used to fill in incomplete data for the 
six- and 12-month measurement. For smoking abstinence, a negative scenario was used to replace missing values, in which respondents who did not fill in the follow-up measurement were regarded as smokers.

\section{Costs and effects at the 12 month follow-up}

Mean costs of the three groups were compared using nonparametric bootstrapping (5000 times) with 95\% confidence intervals in percentiles (Drummond et al., 2005). One-way ANOVAs and Chi-square tests were conducted in order to investigate whether any differences existed between the groups regarding costs and effects after 12 months follow-up.

\section{Cost-effectiveness and cost-utility analyses}

In order to compare the cost-effectiveness and cost-utility of the three conditions, an incremental cost-effectiveness ratio (ICER) was calculated (probability of smoking abstinence/QALYS). For quality of life, ICERs are often called incremental cost-utility ratios (ICURs). However, since ICERs and ICURs can only compare two groups, a net monetary benefit (NMB) had to be calculated in order to compare the cost-effectiveness and cost-utility of the three conditions in our study (Stinnett and Mullahy, 1998). The NMB was calculated by valuing the effectiveness and utility outcomes in monetary values using a threshold for society's willingness to pay. In the Netherlands, cut-off points for preventive interventions have been established for the willingness to pay, varying from $€ 0$ to $€ 80,000$ (RVZ, 2006). A value of $€ 18,000$ is an accepted Dutch cut-off point for the willingness to pay per QALYs (RVZ, 2006); therefore, for both outcome measures (smoking abstinence and QALY) we present the monetary threshold, ranging from $€ 0$ to $€ 18,000$ to $€ 80,000$.

\section{Sensitivity analyses}

In order to deal with uncertainty around the estimates of cost-effectiveness and cost-utility, a nonparametric bootstrap resampling technique was used with 1,000 replacements. Random samples were drawn from the original dataset resulting in 1,000 different samples and accompanying ICERs (Drummond et al., 2005, Glick et al., 2007). Percentages were calculated so that the 1,000 slightly different ICERs resulted in a certain outcome. Four different outcomes were possible: 1) more effects and lower costs (dominant); 2) fewer effects and lower costs; 3) more effects and higher costs; or 4) fewer effects and higher costs (inferior). The resulting decision uncertainty is graphically presented by a cost-effectiveness acceptability curve (CEAC) and a cost-utility acceptability curve (CUAC). In addition, different sensitivity analyses were executed to control for possible uncertainty of parameter estimates during the primary analyses: (1) we used seven-day point prevalence abstinence as outcome variable (instead of prolonged abstinence); (2) we excluded all surgery costs since these costs were extremely high compared to the other health-care-related costs; (3) we tested the results from a health-care 
perspective and excluded the respondent costs and productivity costs because these might be reflected in the respondents' reported quality of life (Hakkaart-van Roijen et al., 2010); (4) we excluded all medication costs, because these were very high compared to the other societal costs.

Data were analysed using SPSS 19.0 (SPSS, Inc., Chicago, IL. USA). Microsoft Office Excel 2010 was used for all bootstrap analyses.

\section{Data availability}

We would like to acknowledge a recently made plea for full disclosure to maximize scrutiny, foster accurate replication, and facilitate future data syntheses (Crutzen et al., 2012). Therefore the Excel files, including bootstrapping algorithms, are available at https://osf.io/hcwj3/files/.

\section{Results}

\section{Baseline characteristics}

Of the 2,099 respondents who were eligible for the current study, 670 were randomized to the video-based condition, 708 to the text-based condition and 721 to the control condition. Table 1 shows the baseline characteristics of respondents in each of the three conditions, as well as their health-care-related costs and productivity costs over three months prior to baseline. Respondents had a mean age of 45.7 years (SD 12.8), 1,278 (60.9\%) of them were female and 705 (33.6\%) had a lower level of education. No significant baseline differences were found between the three groups.

Dropout analyses showed that respondents were more likely to return to the study when they were older $(O R=1.02, p=.000)$ and when they had Dutch nationality $(O R=1.62, p$ $=.02$ ). After the imputation of missing values, total cost data were available for 2,082 respondents (99.2\%), whereas effect data were available for 2,099 respondents (100\%) for the two abstinence measures (prolonged abstinence and seven-day point prevalence abstinence) and 2,088 (99.5\%) for the QALYs.

\section{Costs and effects at the 12 months follow-up}

Table 2 shows the mean societal costs for the three conditions over a period of one year. Since the experimental conditions were the most time-intensive conditions, the time costs were significantly higher than in the control condition. No significant differences were found between the three conditions with regard to health-care-related costs and productivity costs. Furthermore, no significant group differences were found in QALYs (Table 3).

A significant difference was found between the three conditions regarding the effects of the interventions on prolonged abstinence $\left(\chi^{2}=6.13, \mathrm{df}=2, p=.05\right)$ (see Table 3). Pairwise 
comparisons between the different groups revealed that the video-based condition significantly differed from the control condition $(F(2,2,096)=3.07, p=.05)$. A higher proportion of respondents in the video-based condition achieved prolonged abstinence than in the control condition.

\section{Cost-effectiveness and cost-utility analyses}

Table 4 presents the incremental costs and effects per prolonged abstinent smoker and per QALY gained. Analyses showed that the video-based condition resulted in slightly higher costs, but also in more effects. From a willingness to pay of $€ 1,500$ or higher, the video-based condition appeared to be more likely to be cost-effective than the control condition. From a willingness to pay of $€ 50,400$ or higher, the text-based condition seemed to be more costeffective than the control condition. When costs and effects were compared between the two experimental conditions (video-vs. text-based computer tailoring), the video-based condition dominated the text-based condition. Costs in the text-based condition were higher, whereas effects were lower than in the video-based condition.

The probability that the video-based condition was cost-effective at a willingness to pay of $€ 0.00$ per abstinent respondent was $42 \%$, for the text-based condition $16 \%$ and for the control condition $42 \%$. With a willingness to pay of $€ 18,000$ per abstinent respondent, the video-based condition would probably be the most cost-effective (i.e. 70\%), followed by the control condition (i.e. 20\%) and the text-based condition (i.e. 11\%). With increasing the threshold value up to $€ 80,000$, again the video-based condition was the most effective treatment, followed by the text-based condition (i.e. 7\%) and control condition (i.e. 3\%). The probability of each treatment being more cost-effective than the two other treatments is also presented in the CEAC (see Figure 3). In secondary analyses, when seven-day point prevalence abstinence was used as outcome measure (see Table 5 and Figure 3), comparable results were found. Here again, the video condition was the most cost-effective treatment (i.e. > 38\%, > $46 \%$ and $>48 \%$ with a willingness to pay of $€ 0.00, € 18,000$ and $€ 80,000$, respectively). All other sensitivity analyses yielded a similar pattern (see Table 5 and Figure 3). 


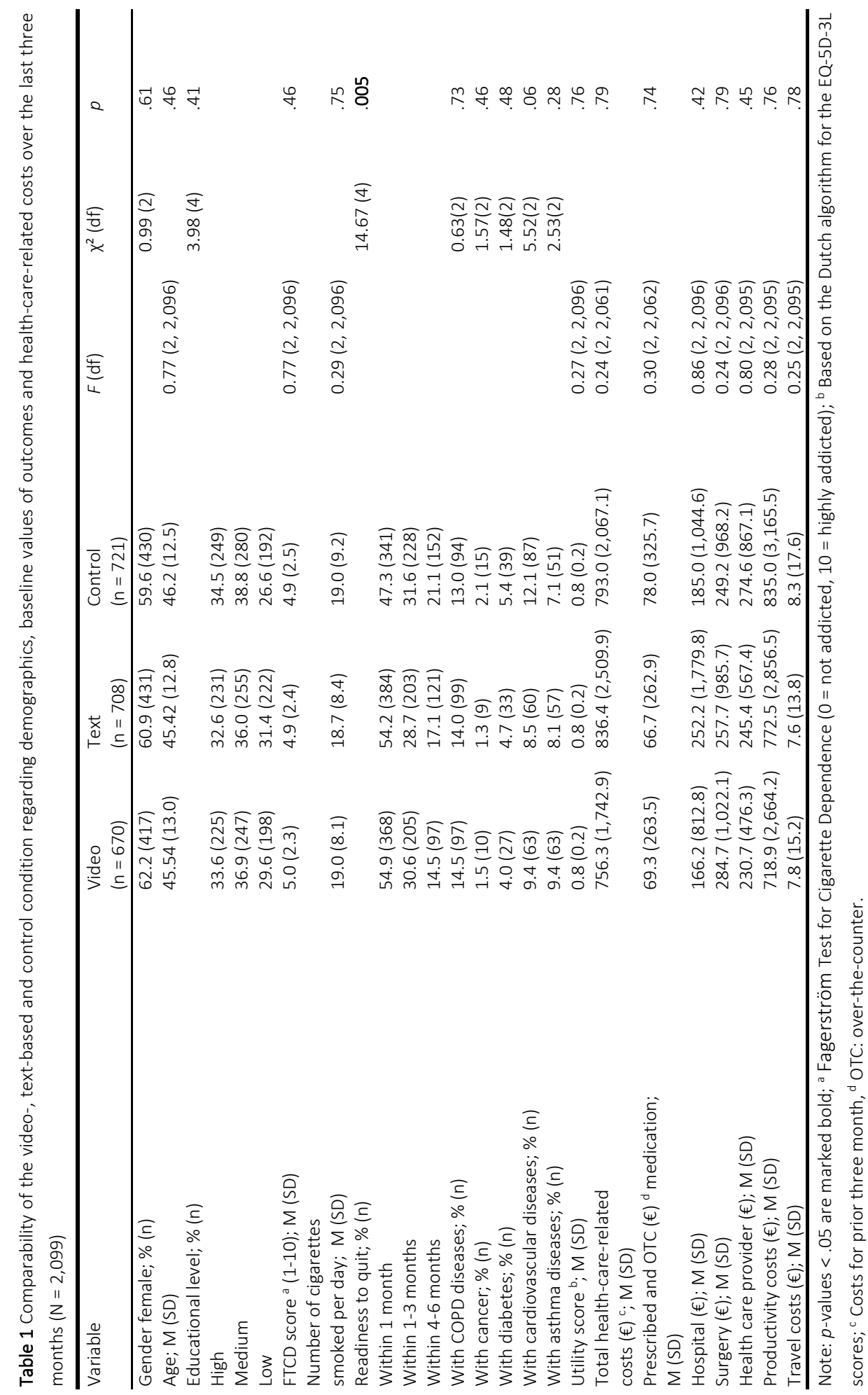




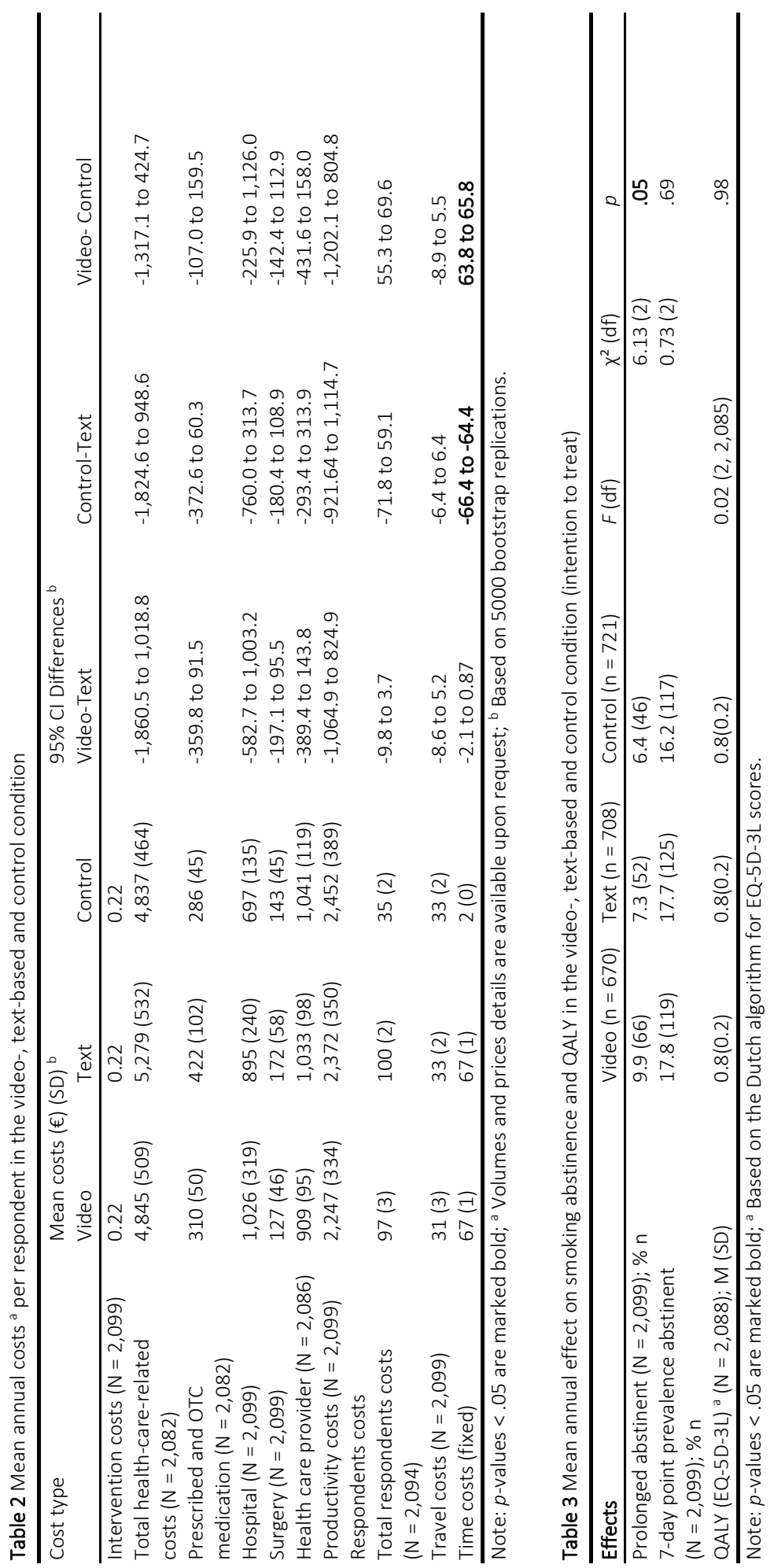




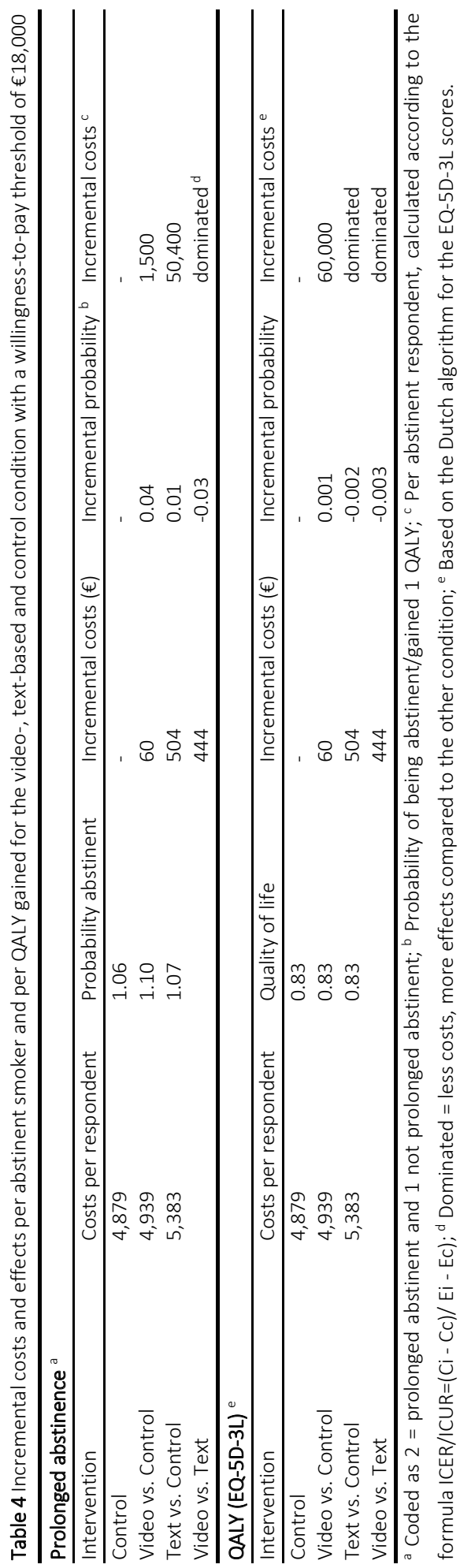




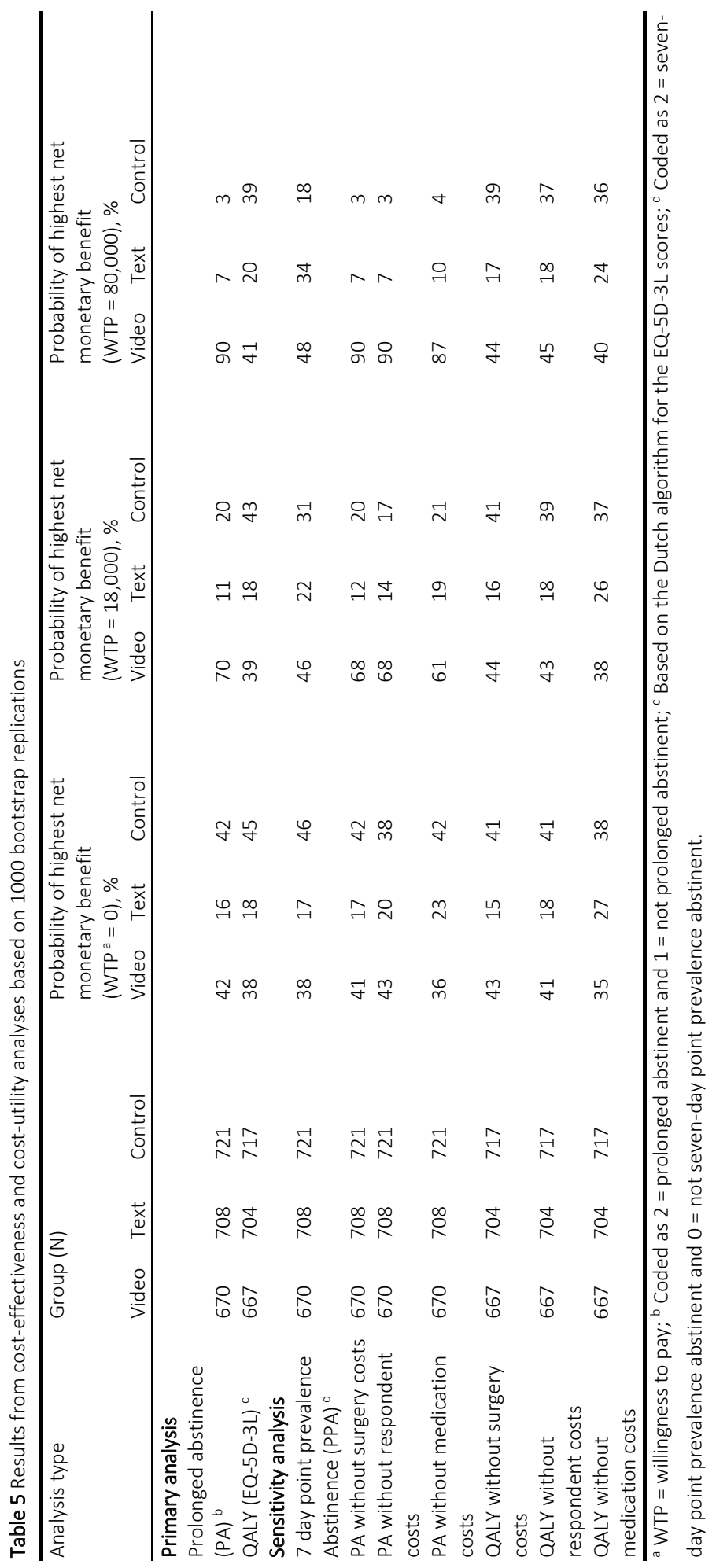




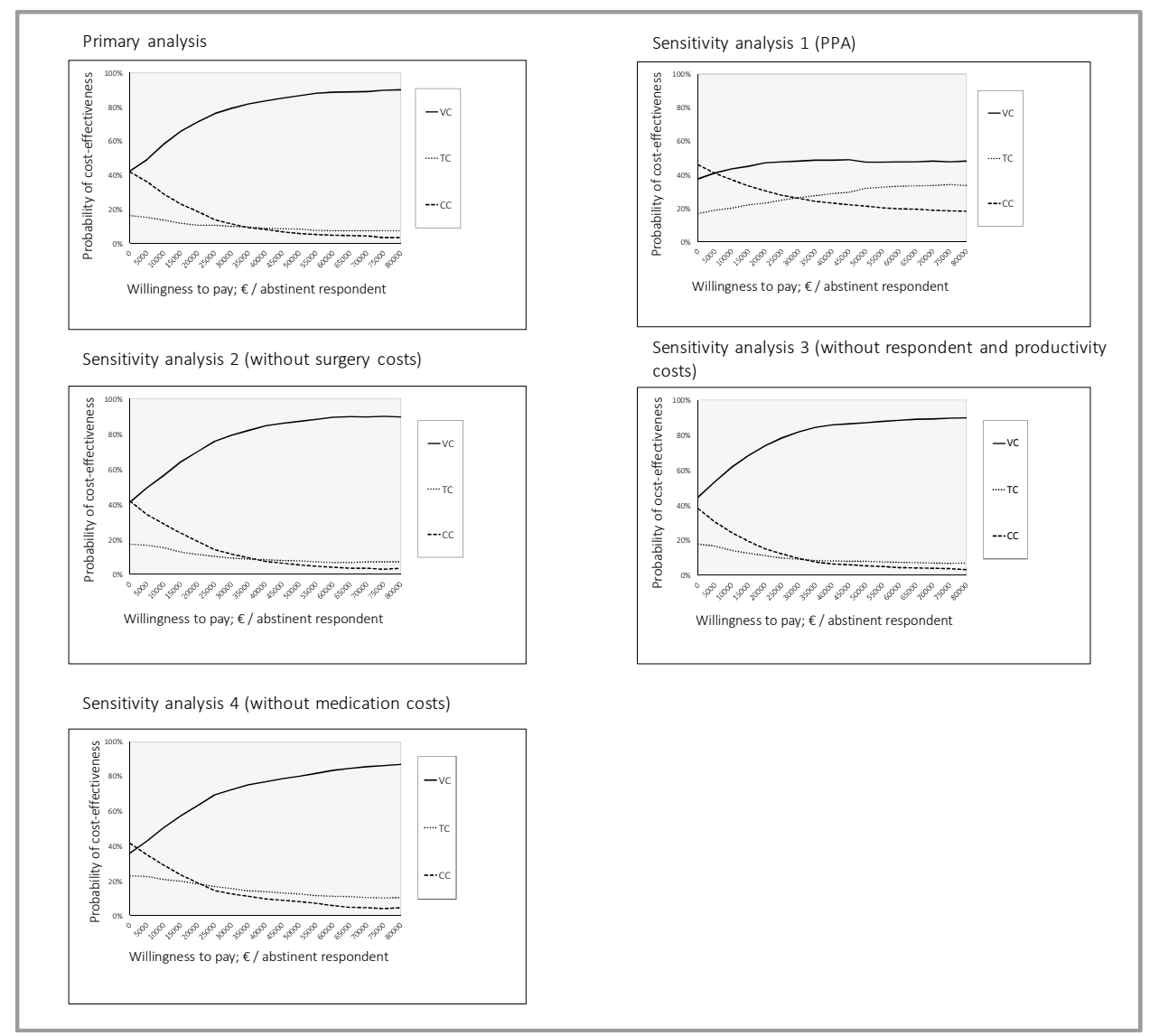

Figure 3 Primary and sensitivity analyses of cost-effectiveness acceptability curve for the three conditions: videobased, text-based and control condition

Results from the cost-utility analyses showed that the text-based condition was dominated by the control condition, since this treatment was less effective and more expensive (see Table 4). Furthermore, results revealed that the video-based condition was more expensive and more effective than the control condition in increasing the number of QALYs gained. When comparing the video-based condition with the text-based condition, costs were lower and effects were higher regarding QALYs gained within the video-based condition. Therefore, the text-based condition was dominated by the video-based condition.

The probability that the control condition was efficient at a willingness to pay of $€ 0.00$ per QALY was 45\%, for the video-based condition 38\%, and for the control condition $18 \%$. With a willingness to pay of $€ 18,000$ per QALY, the control condition would probably be the most efficient treatment (i.e. 43\%), followed by the video-based condition (i.e. 39\%) and the textbased condition (i.e. 18\%). However, increasing the threshold value up to $€ 80,000$, the video- 
based condition would probably be the most efficient treatment (i.e. 41\%), followed by the control condition (i.e. 39\%) and text-based condition (i.e. 20\%). All sensitivity analyses showed a similar pattern (see Table 5). The CUAC for primary and sensitivity analyses are presented in Figure 4.

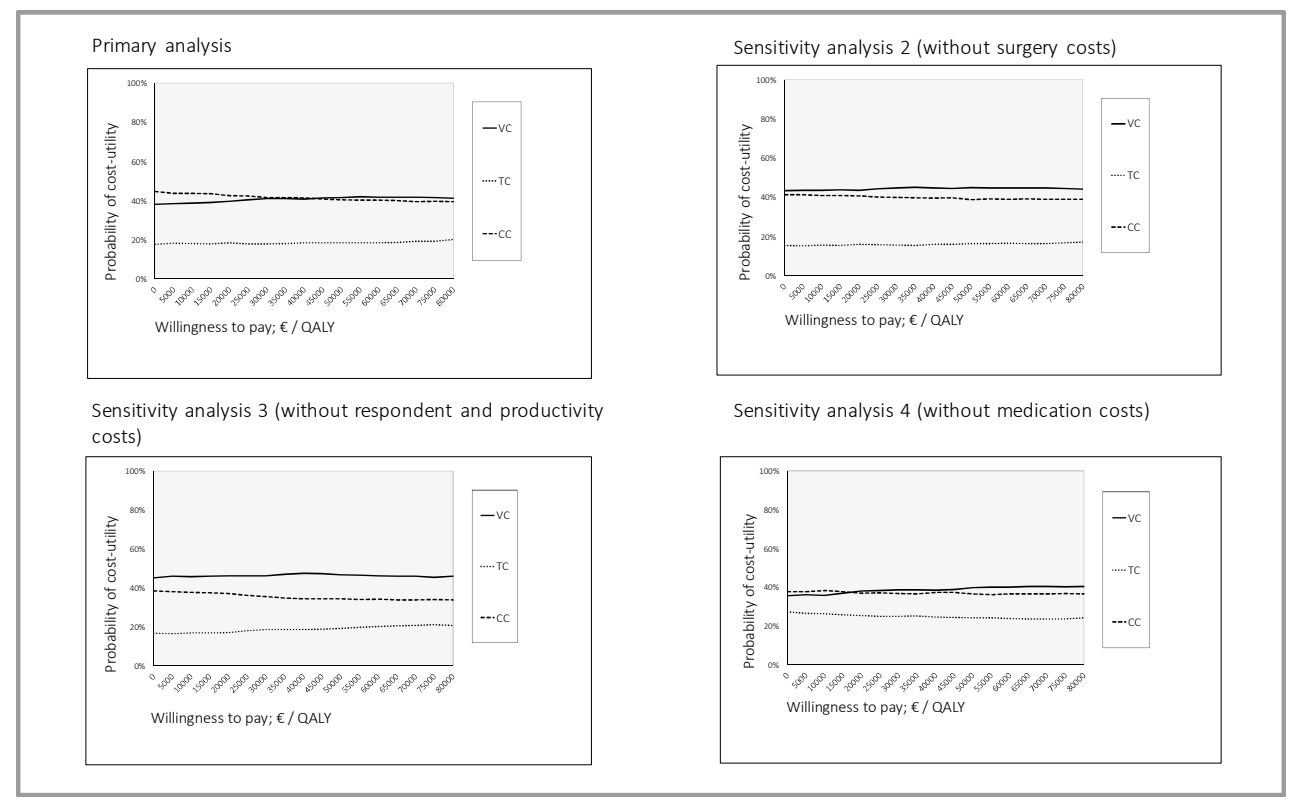

Figure 4 Primary and sensitivity analyses of cost-utility acceptability curve for the three conditions: video-based, text-based and control condition

\section{Discussion}

\section{Main findings}

Until now, there have been only a limited number of economic evaluations of web-based smoking cessation interventions conducted from a broad societal perspective (Smit et al., 2013). In addition, even less is known regarding the relative cost-effectiveness and cost-utility of different delivery methods for web-based computer-tailored smoking cessation interventions (Chen et al., 2012). Therefore, the aim of this study was to investigate the costeffectiveness and cost-utility of two computer-tailored smoking cessation interventions (videovs. text-based computer tailoring), delivered via the Internet. The results of the present study revealed that the video-based computer-tailored smoking cessation intervention was the most cost-effective treatment for smoking abstinence assessed after 12 months from a willingness to pay of $€ 18,000$. Varying monetary threshold values up to $€ 80,000$ showed a similar pattern. Findings suggest that the video-based intervention was far more cost-effective than the textbased intervention and the brief generic advice that respondents received in the control 
condition, since it resulted in more quitters and lower societal costs. The cost-utility analyses with quality of life as outcome measure revealed a different pattern. The QALYs gained by the video-based condition during the one year follow-up period were almost the same as those gained among respondents in the control condition, with far greater costs in the video-based computer-tailored intervention. Consequently, in terms of QALYs, the control condition seemed to be the most preferable treatment when using a monetary threshold of $€ 0$ to $€ 18,000$. When increasing the threshold value for an additional QALY up to $€ 80,000$, the results changed and showed a preference for the video-based condition. The results from sensitivity analyses revealed comparable results. When cost estimations varied, the video-based computer-tailored intervention remained the most cost-effective treatment, whereas costutility was still the highest within the control condition.

Consistent with findings from other economic evaluations of smoking cessation interventions (Smit et al., 2013, Song et al., 2002, Woolacott et al., 2002), our results indicated that the video-based computer-tailored smoking cessation intervention is likely to be most cost-effective. Compared to the control condition, $€ 1,500$ had to be paid within the videobased intervention per abstinent respondent. This amount is somewhat lower than what was found in previous research (Salize et al., 2009, Smit et al., 2013). Nevertheless, these results are difficult to interpret since little information is available on the amount of money that society is willing to pay for smoking cessation and as a result no accepted cut-off points exist for smoking abstinence rates (Smit et al., 2013).

Since difficulties may arise when policy makers try to compare results from costeffectiveness analyses in which the cost-effectiveness ratios are expressed as concerning different outcome measures, we furthermore included the cost-utility analyses to our study. With regard to cost-utilities, if society is willing to pay only a small amount per QALY gained, our results showed that the control group might be the most preferable treatment compared to both computer-tailored smoking cessation interventions. Comparable results were found in a previous study (Smit et al., 2013), where the control condition (usual smoking cessation care in the general practice setting) was also more preferable compared to a computer-tailored smoking cessation intervention and a computer-tailored smoking cessation intervention with face-to-face counseling by a practice nurse. One possible reason for this finding might be that the follow-up period of 12 months was not long enough to observe improvements in quality of life. Previous economic evaluations in respondents with smoking-related diseases indicated that quality of life could only be detected with longer time periods (Ladapo et al., 2011, QuistPaulsen et al., 2006). One possible explanation for the different results of the costeffectiveness and cost-utility analyses could be that respondents may not have perceived improvements in their quality of life during the one-year follow-up period used in the present study. Since we used a relatively short follow-up period of 12 months, respondents may not have perceived any direct health benefits in the short term, due to possible withdrawal 
symptoms associated with quitting (Shiffman et al., 2006, Wiggers et al., 2006). Although, other studies did identify a positive association between smoking abstinence and quality of life yet over a 12-month follow-up period (Hays et al., 2012, Sales et al., 2009), future economic evaluations should include longer time horizons in order to detect an improved quality of life for respondents who quit smoking.

\section{Strengths and limitations}

To our current knowledge, this was one of the first studies examining the cost-effectiveness and cost-utility of a video- and text-based computer-tailored intervention for smoking cessation. In order to compare the three different conditions more specifically, the effects of the current study were not only assessed in terms of quality of life but also in terms of smoking abstinence. The inclusion of this cost-effectiveness analysis (using smoking abstinence as outcome measure) allows policy makers to compare the net benefit of the intervention to other smoking cessation interventions. Moreover, several sensitivity analyses were conducted to test for uncertainty of parameter estimates which can be seen as another strength of our study.

Yet, several limitations of the current study should also be discussed. First, we interpreted our results at varying monetary threshold values of smoking abstinence and QALY up to $€ 80,000$. However, until now no information has been available on the exact amount of money that society is willing to pay for smoking cessation, i.e. per additional ex-smoker, which makes interpretation difficult. Although the willingness to pay of $€ 18,000$ is an accepted Dutchcutoff point for measuring the QALY (Casparie et al., 1998), past research has suggested varying these willingness to pay values (Gerhards et al., 2010). Nevertheless, we assume that the chosen range of our study from $€ 0$ to $€ 80,000$ was broad enough to capture all important threshold values. As already recommended by previous research (Smit et al., 2013), future studies should however aim to determine a cut-off point for the willingness to pay per abstinent respondent in order to enable an accurate interpretation of the findings from the present and similar studies. Second, we were not able to detect improvements in quality of life between the different conditions. For future economic evaluations regarding smoking cessation interventions, it may be useful to include other operationalisations of the QALY or other outcomes, which may be better able to detect short-term health changes (e.g. nonhealth-care-related outcomes such as satisfaction or enablement) (Chalkidou et al., 2008, Lorgelly et al., 2010). Third, health-care utilization was based on self-reported data, which might have introduced recall bias. However, we tried to keep recall bias low by using a threemonth instead of six-month retrospective questionnaire (Hassan, 2006, Margetts et al., 2003). Upcoming economic evaluation studies may, however, want to include more objective measurements (such as data from health insurance, or medication registration from the pharmacy or medical professions) of health-care utilization. 


\section{Conclusion}

The results of our study revealed that the video-based computer-tailored smoking cessation intervention had the highest probability of being cost-effective. Yet, the cost-utility outcomes tended to be in favour of the control condition, but interpretation of this finding is hindered by the relatively short follow-up and great likelihood of insensitivity of the QALY assessment. Future studies need to assess which QALY measure may be the most sensible method to detect short-term improvements regarding quality of life. Finally, more research is needed to identify an acceptable cutoff-point for the willingness to pay per abstinent respondent in order to interpret incremental costs in future studies concerning the cost-effectiveness of smoking cessation interventions. 



\section{CHAPTER 8}

General discussion

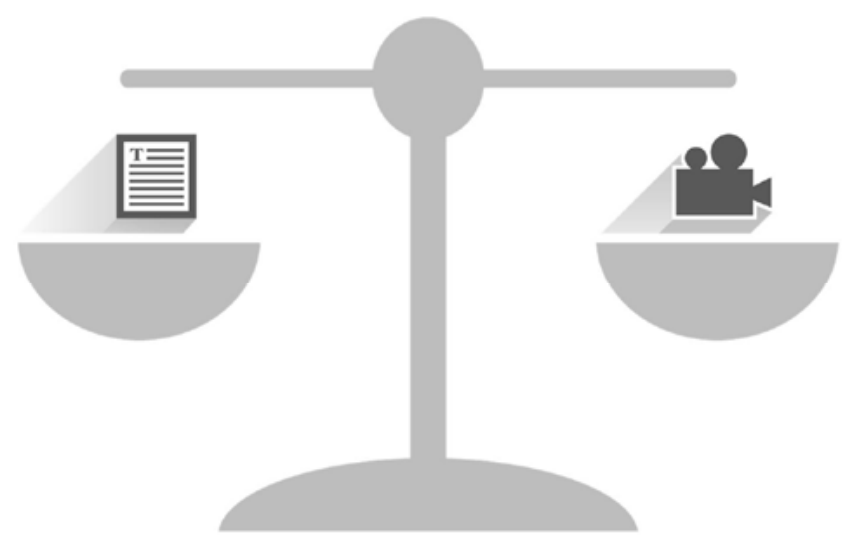



The main objective of this dissertation was to test two web-based computer-tailored smoking cessation interventions, a video-based and text-based multiple computer-tailored intervention with different motivational routings, aimed at various socioeconomic status (SES) groups, with regard to effectiveness and cost-effectiveness. Additionally, this dissertation aimed to provide more insight into the reach, adherence and processing of the two computer-tailored smoking cessation interventions among different socioeconomic groups. In this chapter, all main findings will be summarised and will be related to previous research in this area. Furthermore, several methodological and practical considerations will be discussed; in addition, recommendations for future research and practice will be given. The chapter will end with a general conclusion.

\section{Summary and discussion of the main findings}

\section{Reach of the computer-tailored smoking cessation interventions}

The study presented in Chapter 3 investigated the reach, retention and costs of four different strategies (i.e. recruitment through General practitioners (GPs), newspapers, Internet, and other strategies such as mailing organisations) aimed to recruit smokers with different SES levels for participation in the computer-tailored smoking cessation intervention (SES was indexed by educational level).

Recruitment via GPs reached a relatively large proportion of lower educated smokers and smokers with smoking-related diseases. Nevertheless, in absolute numbers, more smokers were reached by newspapers, Internet and mailing strategies. Furthermore, retention rates were higher among those recruited by GPs compared to those recruited through other strategies. Yet, this applied only to respondents with a lower readiness to quit smoking (readiness to quit within two months and five years). Results, furthermore, suggested that highly addicted respondents recruited via newspapers were less likely to make a quit attempt compared to highly addicted respondents recruited via GPs or the Internet. Concerning smoking abstinence, females recruited via newspapers were less likely to be abstinent compared to females recruited via GPs. No significant differences were found between different SES groups regarding retention and smoking behaviour. Finally, the recruitment costs via GPs were the highest compared with the other three strategies.

Although earlier studies often reported problems in engaging and retaining lower socioeconomic status (LSES) groups in particular (Brouwer et al., 2010, Businelle et al., 2010, Eysenbach, 2005), our study showed that retention rates did not differ between LSES, middle and higher socioeconomic status (MSES and HSES) smokers. Additionally, in line with previous research (Belisario et al., 2012, Hoving et al., 2007, Smit et al., 2011), our findings showed that recruitment via GPs resulted in a relatively larger population of smokers who participated and made a successful quit attempt compared with recruitment via mass media strategies (such as 
newspaper advertisement). A possible explanation could be that respondents recruited via GPS were more motivated and involved in quitting compared to the more 'cold-contacted' groups who were recruited by mass media strategies (e.g. newspapers) and who may have become, by chance, aware of the program (Balmford et al., 2008, Balmford et al., 2013). Although the findings confirm that recruitment via GPS was especially successful in reaching LSES smokers who made a successful quit attempt, the net effect in quitters was still more in favour of the mass media approach in which a larger absolute number of (LSES) smokers were recruited. In line with similar findings found in previous research (Smit et al., 2011), the results are inconsistent with the idea that mass media approaches are less successful in recruiting smokers with a LSES (Dalstra et al., 2002, Marmot, 2005). As a result, the public health impact of the intervention might be higher when the potential target group is recruited via a mass media approach since more smokers were reached by lower costs compared to the other strategies (Graham et al., 2008). In sum, recruitment of smokers via mass media channels (such as newspapers and the Internet) seems to be a better and lower-priced approach for reaching a higher number of smokers for future web-based smoking cessation interventions.

\section{Future adherence and processing towards the computer-tailored smoking cessation intervention} The study presented in Chapter 4 investigated whether differences exist in message-processing mechanisms (i.e. attention, comprehension, self-reference, appreciation and processing towards the computer-tailored messages) and future adherence (i.e. intention to visit/use the Intervention website again; recommend the website to others), according to delivery strategy (video- vs. text-based messages) and SES (SES; indexed by educational level) towards the smoking cessation intervention.

Delivery strategy was found to have no influence on future adherence and processing mechanisms and did not differ between HSES and LSES smokers. However, in both conditions (video-based vs. text-based messages) results showed that LSES smokers paid significantly more attention to the tailored messages and seemed to process the information more deeply than HSES smokers. Additionally, results revealed that LSES smokers were more inclined to revisit the intervention website compared to HSES smokers.

The idea that processing of the computer-tailored information and future adherence towards the intervention would depend on the delivery strategy did not hold for this study and is in line with previous findings (Vandelanotte et al., 2012). A first explanation could be the limited exposure time. During this study, respondents had to follow only one session of the intervention (total intervention has three to six sessions, depending on smoker's readiness to quit smoking). It is therefore conceivable that the delivery mode would have influenced revisits when respondents had followed more sessions. A second explanation could be that we recruited respondents via an Internet research agency, which may have, in general, more innovative members. For these people, delivery mode may not make a difference. If 
respondents were recruited by other strategies (e.g. newspaper advertisement or GP practices (Chapter 3, 5 and 6)), delivery mode preferences may have been different. Third, it is possible that the recruited sample was not enthusiastic about the design of the intervention components. It is possible that the experimental conditions (video- vs. text-based messages) may have been offered in a more engaging way with other elements such as graphics, cartoons and animation effects. Finally, a further potential explanation could be related to the motivational level of respondents. Respondents recruited via the Internet research agency may have been less motivated to quit smoking because they did not actively search for a smoking cessation program and may not have felt the need to process the tailored information, which in turn may have led to adverse effects of our delivery strategy. Furthermore, findings of the study confirm that LSES smokers were more inclined to revisit the intervention website compared to HSES smokers. These findings correspond with a previous study that found that lower educated respondents were more likely to finish a component of a computer-tailored life style intervention (Schneider et al., 2012). In sum, our findings confirm that lower educated smokers more positively evaluated the given tailored advice. Although findings of this study should be interpreted with caution since we did not measure actual revisits; the results indicated that the intervention succeeded in approaching LSES smokers in the population who might profit the most from these computer-tailored interventions.

\section{Effectiveness of the two computer-tailored smoking cessation interventions}

Respondents willing to quit within six months received tailored feedback over a period of three months in the text- and video-based intervention. In the text computer-tailored (TCT) condition, respondents received multiple sessions of text-based advice without any graphics or animations. In the video computer-tailored (VCT) condition, the same tailored advice was presented by adults in a video message. In both interventions, respondents with different levels of motivation were directed to one of the two motivational routings and received tailored feedback, adapted to their readiness to quit smoking. The effectiveness of the two computer-tailored interventions was compared to a control condition (in which respondents received a short text advice after baseline) after six and 12 months.

Data of the six- and 12-month follow-up measurements were analysed using three methods. The first method implied a negative scenario, where respondents who did not return to the follow-up measurement were regarded as smokers. The second method entailed a multiple imputation approach in which missing values for the outcome variables were imputed based on the regression of all other relevant variables (Schafer, 1999). Third, complete case analyses were performed, including only respondents who completed the follow-up measurement. Since not all respondents in the experimental conditions adhered to all intervention elements, the inclusion of these respondents in the effect analyses might distort the assessment of an intervention's effectiveness. For assessing the six-month effects, we 
therefore analysed two different samples (Elfeddali et al., 2012a). The first sample included all randomly assigned respondents that completed baseline questionnaires and session 1 (directly after baseline; including setting a quit date). The second sample included only respondents in the experimental conditions who at least finished one further session of the two different routings of the intervention.

Chapter 5 described the results six months after baseline. When seven-day point prevalence was used as an outcome measure in the most conservative negative scenario, no interaction effect was found between SES (SES; indexed by educational level (categorized into LSES, MSES and HSES)) and condition nor between readiness to quit smoking (motivation/intention to quit) and condition (main analyses). However, in sample 1, the videobased condition was more effective than the control condition in increasing seven-day point prevalence abstinence (VCT: 20.9\%; TCT: 17.9\%; CC: 14.6\%). In sample 2, both conditions were significantly more effective than the CC (VCT: 30.6\%; TCT: 22.6\%; CC: 14.6\%). Additional analyses with the multiple imputation dataset and complete cases revealed similar findings in favour of the video-based intervention.

When prolonged abstinence was used as outcome measure (also negative scenario), an interaction effect was found between motivation to quit smoking and the conditions. In sample 1, subsequent subgroup analysis showed that the video-based computer-tailored intervention resulted in significantly higher abstinence rates compared to the control condition among respondents with a lower motivation to quit smoking (within four to six months) (VCT: 14.4\%; TCT: 8.3\%; CC: 3.3\%). In sample 2, comparable results were found. The video-based computer-tailored intervention resulted in higher abstinence rates compared to the control condition among respondents with a low readiness to quit (intending to quit within four to six months) (VCT: 23.1\%; TCT: 9.5\%; CC: 3.3\%) and high readiness to quit (intending to quit within one month) (VCT: 25.1\%; TCT: 21.4\%; CC: 15.2\%). The multiple imputation analyses, as well as the complete case analyses, yielded comparable results in which the video-based computertailored intervention was more effective compared to the control condition in smokers with a lower readiness to quit smoking. Additionally, we found significantly higher abstinence rates among those respondents who adhered to more than one session in the video-based condition across all educational groups.

Chapter 6 described the results 12 months after baseline. Again no interaction effects between SES (SES; indexed by educational level (LSES, MSES and HSES) and a combined measure of occupational status with educational level) and condition and readiness to quit smoking and condition were found. However, we found significant overall effects of the videobased computer-tailored intervention on prolonged abstinence, using multiple imputation analyses (main analyses). Complete case analyses revealed comparable findings to the multiple imputation approach (VCT: 20.2\%; TCT: 13.5\%; CC: 12.0\%). In the negative scenario approach, the video-based computer-tailored intervention still reported higher prolonged abstinence 
rates compared to the control condition (VCT: 9.9\%; TCT: 7.3\%; CC: 6.4\%), though differences did not reach significance. When we used seven-day point prevalence as an outcome measure, we were not able to detect significant differences between conditions.

While we expected positive effects for the video-based computer-tailored intervention on smoking abstinence for LSES smokers and positive effects for the text-based computertailored intervention for HSES smokers, no significant differences in quit rates were found in the two experimental conditions between LSES, MSES and HSES smokers. Whereas many health promotion interventions have the negative side effect that they contribute to an increase in health disparities (Bauld et al., 2007, Mackenbach et al., 2003), the findings of our studies showed that the tailored video-based intervention was equally effective for all socioeconomic groups of smokers. In line with previous research in other health behaviour related fields (Campbell et al., 1999, Houts et al., 2006, Tuong et al., 2012), respondents are likely to profit the most from video-based information. A possible explanation for the finding that LSES, MSES as well as HSES smokers profited from the video-based intervention could be that Internet users have a general preference towards video information. Since more and more websites make use of video content (Purcell, 2010) and most of LSES, MSES and HSES Internet users are probably used to receive video-based information, they might have developed a general preference towards video information compared to information that is presented in plain text format (Purcell, 2010). In line with this, recent research indicated that Internet users support the concept of video-tailored information above text-based information (Calvert et al., 2005, Vandelanotte and Mummery, 2011, Wilson et al., 2010). Moreover, it is possible that smokers were better able to process the video-tailored messages compared to text-based messages. As video-based information has shown to require less mental effort and our program evaluation also showed a slightly better processing of the video-based messages (although not significant), it is likely that, in general, smokers better understood and processed the video tailored advice compared to the text tailored advice. Furthermore, although the textbased intervention was effective in respondents who adhered to more than one session of the intervention (after six months), a potential shortcoming of this intervention may be that it did not include any other elements, such as graphics, illustrations or cartoons. We can assume that respondents in the text-based condition were not engaged by the design of the intervention, which in turn, may have decreased the overall effectiveness of the text-based condition.

Additionally, a benefit of the video-based intervention for respondents with a high (ready to quit within one month) and low motivation to quit (ready to quit within four to six months) was observed in our trial after six months. The video-based computer-tailored intervention was not only successful in stimulating smoking cessation in highly motivated smokers but also in smokers with a lower motivation to quit. Although we did not assess the unique effects of the two motivational routings on smoking cessation among different subgroups of smokers, we posit that it is likely that lower motivated smokers in the video-based 
intervention profited from the special motivational routing in which they got the opportunity to reflect on their smoking behaviour before deciding on their quit attempt and were acknowledged in their unwillingness to quit in the short-term without any pressure to quit. Although the text-based condition likewise included motivational routings, a possible explanation why this intervention was less effective may be that respondents were more exhausted by reading their advice. An explanation could be that the text-based messages demanded more cognitive effort, which in turn may have led to adverse effects among smokers with different motivational levels. Furthermore, as mentioned already, respondents might have been less attracted by the design of the text-based messages.

Furthermore, the video-based intervention was ineffective for smokers with a moderate motivation to quit smoking (ready to quit within one to three months) in the six months effect measurement. During our intervention, stage-based tailoring was used in which smokers in preparation (intending to quit within a month) received different feedback than smokers in contemplation (intending to quit within two to six months) (De Vries et al., 2003, De Vries and Mudde, 1998). Past research has already suggested that the contemplation phase may entail a mixture of smokers who are high to moderate in their motivation to quit (Herzog, 2008). A possible explanation could be that those smokers with a moderate motivation to quit, who were directed to routing 2 , faced different barriers and problems compared to smokers with a lower motivation to quit. Since they already had a relatively high intention to quit smoking, they may have profited less from routing 2 , in which smokers were able to reflect on their intention to quit smoking.

\section{Cost-effectiveness of the two computer-tailored smoking cessation interventions}

Results from the cost-effectiveness study after the 12-month follow-up showed that the videobased computer-tailored intervention was the most cost-effective treatment for smoking abstinence, varying the willingness to pay per abstinent respondent from $€ 0$ up to $€ 80,000$ (Chapter 7). From a willingness to pay of $€ 1,500$, the video-based intervention was likely to be the most cost-effective treatment whereas from a willingness to pay of $€ 50,400$, the text-based intervention was likely to be the most cost-effective after 12 months. Thus, the video-based computer-tailored intervention showed higher effects on smoking abstinence and lower costs compared to the text-based intervention. Regarding cost-utilities, when quality of life was used as an outcome measure, the control condition seemed to be the most preferable treatment. Sensitivity analyses, for which costs estimations varied, revealed comparable results to the cost-effectiveness and cost-utility analyses.

In line with findings of previous economic evaluations of smoking cessation interventions (Smit et al., 2013, Song et al., 2002), the results showed that the video-based computer-tailored intervention was the most cost-effective option compared to usual smoking cessation care. Compared to the control condition, the costs per abstinent respondents were 
$€ 1,500$ within the video-based condition and were slightly lower compared to what was found in previous studies (Salize et al., 2009, Smit et al., 2013). In the Netherlands, a willingness to pay of $€ 18,000$ per quality adjusted life years (QALY) is an accepted cut-off point when it concerns preventive interventions (Casparie et al., 1998). Yet, up to now, no information is available on the societal willingness to pay per abstinent smoker; this makes interpretation of the results in terms of clinical implications difficult. Moreover, the results of our cost-utility analysis revealed that care as usual (short generic text advice) was the most efficient treatment and is comparable to previous findings (Smit et al., 2013). An explanation for this finding could be that the relatively short follow-up period of 12 months was possibly not long enough to detect changes and improvements in quality of life. Past research on respondents with smoking-related diseases showed that relevant changes in quality of life are often detected with longer time periods (Ladapo et al., 2011, Quist-Paulsen et al., 2006). Perhaps, more time might be needed to assess changes and improvements in quality of life. Another topic relates to the differences we found between cost-effectiveness and cost-utility analyses. A possible explanation could be that respondents may not have perceived any direct health benefits in the short-term (after 12 months) due to nicotine withdrawal symptoms related to their quit attempt (Wiggers et al., 2006). Difficulties in refraining from smoking and several health problems due to withdrawal may have diminished improvements in quality of life (Shiffman et al., 2006).

\section{Methodological and practical considerations}

The results of the studies should be interpreted in light of several methodological and practical considerations that could have affected the validity of the different studies presented in this dissertation. Although different methodological shortcomings were already discussed in earlier chapters, the following paragraphs will summarize the most important methodological and practical concerns.

\section{Study validity}

A randomised controlled trial (RCT) was considered as the most appropriate design for the (cost) effectiveness studies described in this dissertation. The video- and text-based computertailored smoking cessation interventions were compared with a control group, receiving only a short generic text-based advice. It is possible that the external validity of the studies performed in this dissertation may have been limited.

First, a selection bias might have occurred. Those respondents who agreed to take part in our study might have differed from those who did not participate. Due to our inclusion criteria, it is likely that we included a relatively highly motivated group of smokers, causing a potential overestimation of our smoking abstinence outcomes. Since these smokers were 
included in our effect analyses, they could have increased success rates compared to what we can reach in terms of quitting in real life implementation and compared to other studies which included smokers with a lower or no motivation to quit (Chan et al., 2011, Civljak et al., 2013, Oenema et al., 2008). Moreover, selection bias might have also occurred due to our recruitment strategies. As described in Chapter 3 of this dissertation, mode of recruitment influenced the type of smokers participating in the computer-tailored smoking cessation intervention. It was shown that the differently recruited samples differed with regard to their retention and quitting behaviour. Recruitment via GPs reached particularly LSES smokers with more smoking-related diseases (such as chronic obstructive pulmonary disease (COPD)). In addition, the national financial compensation for smoking cessation pharmacotherapy in the Netherlands was introduced (in 2011) during our recruitment period (Willemsen et al., 2013). During this period, smokers received financial reimbursement for smoking cessation pharmacotherapy in combination with behavioural support, which may also have influenced the enrolment of our smokers. For this reason, smokers who wanted to quit with pharmacotherapy may not have chosen to participate in our computer-tailored intervention. The above-mentioned considerations indicate a potentially limited generalisability of our results to other populations of smokers.

\section{Measurement of smoking abstinence and validation}

In most of the studies that are subject of this dissertation we used prolonged abstinence and seven-day point prevalence abstinence as our outcome measures. Since prolonged abstinence is considered to be a better predictor of long-term abstinence than seven-day point prevalence abstinence (Hughes et al., 2003), we used prolonged abstinence as our primary outcome measure in our 12-month effectiveness paper. In our study, prolonged abstinence was defined as being abstinent since the last quit attempt (setting a quit date within the intervention period), allowing a grace period of two weeks in which respondents were permitted to smoke a maximum of five cigarettes (Hughes et al., 2004, West et al., 2005). While prolonged abstinence is able to measure a longer period of abstinence (often several months) and appears to be a conservative measure for smoking abstinence, seven-day point prevalence abstinence is able to include smokers who made a delayed quit attempt or who relapsed, without classifying the respondent as a smoker (Velicer and Prochaska, 2004). Seven-day point prevalence abstinence is therefore able to include a broader range of smokers, including those who just quit before the follow-up measurement and those who were abstinent for several months (Oka et al., 2006). Since seven-day point prevalence is able to include smokers who made a delayed quit attempt not within the intervention period itself, it might have underestimated the intervention's effectiveness and is a possible explanation for why we did not find any effects of seven-day point prevalence abstinence after 12 months. To draw strong 
conclusions about whether smoking abstinence is completely evoked by the effects of the intervention, it would be probably better to report only prolonged abstinence.

One methodological concern is related to the biochemical validation of smoking abstinence. Although self-reported smoking status is seen as a reliable measure in the general population (Patrick et al., 1994, Rebagliato, 2002, Velicer et al., 1992), self-reports could, of course, still lead to biased results (Connor Gorber et al., 2009). Possible reasons for inaccurate self-reports might be the social pressure of other people in the smoker's environment or a feeling of shame that the desired goal (to quit smoking) is not achieved. In the 12-month effectiveness study presented in this dissertation (Chapter 6), which concerns the last followup moment, biochemical cotinine validation was conducted (West et al., 2005). Respondents were asked to test their smoking status themselves, using the NicAlert ${ }^{\circledast}$ test strips at home. Only $4.3 \%$ of the respondents misinterpreted their non-smoking status and were consequently treated as smokers in the effect analysis of this study (a cut-off point of $\geq 2$ for saliva (testing area 1 to 6 ) indicated that respondents still smoked tobacco) (Marrone et al., 2011). Response rates to saliva testing were relatively low compared to other studies (Elfeddali et al., 2012a, Smit et al., 2012), only $37 \%$ of the respondents completed the test and sent the test back to the research team. If self-reported outcomes were not completely truthful in those respondents who did not undergo the test, smoking abstinence may have been slightly overestimated.

\section{Operationalisation of socioeconomic status}

In the majority of studies presented in this dissertation, socioeconomic status was operationalised by respondent's educational level (categorized into LSES, MSES and HSES), which is a frequently used measure to identify social inequalities in the Netherlands and other countries (Elfeddali et al., 2012, Regidor, 2006, Schaap and Kunst, 2009, Smit et al., 2012). Preferably, SES should have been operationalised by several variables, representing different dimensions of SES. Although most of the studies focus on educational inequalities, an alternative strategy is to use other variables such as household income or occupation (Schaap and Kunst, 2009). However, questions about income often show higher non-response rates than other socioeconomic variables and are often less likely to be answered by LSES smokers (Acock, 2005). We tried to collect all these SES variables. Yet, since household income had very high rates of missing values (14.3\%) in our study, we could not utilize it in the operationalisation of socioeconomic status. In the 12-month effectiveness study (Chapter 6), we therefore tested conditional effects of the intervention for different SES groups by taking two socioeconomic measures (i.e. educational level and a combination measure of educational level by occupation) into account. By comparing educational level with occupational status, we were able to investigate whether the combined approach (educational level by occupation) had an additional value above our traditional measure 'educational level.' Results showed that neither 
the combination of educational level by occupation nor educational level alone predicted smoking abstinence. Overall, outcomes indicated that the combined measure (educational level by occupation) had no beneficial effect.

\section{Study drop-out}

Although we made efforts to minimise drop-out by using e-mail reminders and telephone interviews at the follow-up measurements, we still had to deal with drop-out rates of about $30 \%$ and $48 \%$ after six and 12 months, respectively. Although drop-out rates in our study were not extremely high when compared to other web-based smoking cessation interventions (Elfeddali et al., 2012a, Shahab and Mc Ewen, 2009, Smit et al., 2012), they still have some implications and therefore, might bias our results. All three approaches used to deal with missing values on the outcomes in the effectiveness studies (Chapter 5 and 6), have their own weaknesses and strengths. For our main analyses in the two effectiveness studies (Chapter 5 and 6), missing values on the primary outcome measures were replaced, using a negative scenario in which respondents lost to follow-up were regarded as smokers. Although this approach is widely used in order to prevent lack of power, it might be too strict since many non-respondents might actually be quitters (Heritier et al., 2003, Hollis and Campbell, 1999). Whereas the negative scenario might have resulted in an underestimation of the intervention's effectiveness, complete case analyses might have caused an overestimation of the findings (Eysenbach, 2005). Past research has already suggested the use of multiple imputation for the replacement of missing values (Blankers et al., 2010, Schafer, 1999). Besides complete case analyses and the negative scenario analyses, a multiple imputation dataset was therefore used, in which missing values were imputed based on the regression of all relevant variables (Bodner, 2008). During our effect analyses we found similar results with all three approaches, a finding that may be attributed to the fact that rates of missing data were not extremely high. Although the use of multiple imputation seems to be a useful strategy to replace missing values, previous research found that multiple imputation might result in unpredictable estimates when missing values are relatively high (Schulz et al., 2014). Until now, there seems to be no consensus about the best analysis strategy for handling missing data. Therefore, the provision of several approaches, as we did in our papers, may give a broader view of the effects and sheds light on the robustness of the results (Elfeddali et al., 2012a, Schulz et al., 2014, Smit et al., 2012, Te Poel et al., 2009).

Another limitation concerns the selective drop-out. In both effectiveness studies, respondents who were lost to follow-up, were more likely to be younger compared with those who remained in the study. Consistent with previous research (Berndt et al., 2013, Smit et al., 2012), younger smokers might have experienced less smoking-related illnesses and might therefore have been less motivated to change their behaviour. Future research should therefore concentrate on ways to make the interventions more attractive for the younger 
smoking population. Furthermore, drop-out was also determined by nationality. Respondents with a nationality other than Dutch were more likely to drop-out compared with Dutch smokers. A potential explanation could be a barrier of language. Furthermore, respondents who dropped out had furthermore more smokers in their environment and a lower level of self-efficacy. The dropout findings support the idea that smoking cessation interventions should pay more attention to the smoker's ethnical background, the social environment and the smoker's perception of reaching their goal.

\section{Implications and recommendations for future research and practice}

This dissertation investigated the influence of delivery strategy towards a computer-tailored smoking cessation intervention among smokers with different SES and motivational quitting levels. The following section will discuss several implications and recommendations for future research and practice.

\section{Improving the reach of the intervention}

In line with previous research, there is evidence to suggest that recruitment via GPs has the potential to reach more LSES smokers who are often more likely to make a successful quit attempt, as compared to recruitment via mass media strategies (Smit et al., 2011). Proactively identifying smokers (through the GP or other health services) appears to be an effective way of recruiting smokers to smoking cessation interventions (Murray et al., 2009). However, results of the current dissertation also indicate that the net effect in quitters is in favour of the mass media strategies, which often recruit a larger absolute number of smokers. Since the mass media approach reaches a larger number of smokers against lower costs, future research should focus on optimising this approach to better reach LSES smokers who might profit the most from these interventions. Past research has already indicated that LSES smokers appear to be more concerned with their current health status and less triggered by concerns about possible future health issues (Pisinger et al., 2011, Vangeli and West, 2008). As a result, interest in the intervention and motivation to quit may be related to the LSES smoker's current health status (Pisinger et al., 2011). Earlier research suggests that highly emotive advertisements (pictorial messages) could be more effective in getting LSES smokers to participate in a smoking cessation intervention and to make a quit attempt, since this may make health concerns more urgent (Hammond, 2011). Hence, research is needed to confirm whether the use of emotive advertisements may further enhance the reach and the enrolment of LSES smokers in smoking cessation interventions in general and in web-based computer-tailored cessation interventions in particular. 


\section{Optimizing adherence towards the intervention}

Delivery strategy (i.e. tailored feedback via text or video) was not found to influence future adherence to the intervention nor processing mechanisms. Nor did it result in differences on these factors between HSES and LSES smokers. Since dose-response associations are often studied in web-based interventions (Elfeddali et al., 2012a, Verheijden et al., 2007, Zebrack et al., 2005), we conducted further in-depth analyses (results not included in this dissertation) to test whether delivery strategy determined actual adherence and whether this differed between LSES and HSES smokers. Even a previous study suggests that LSES people are often more likely to discontinue the intervention (Nevid et al., 1996), adherence to our smoking cessation interventions did not differ significantly between LSES and HSES smokers.

Although our approach was able to keep LSES smokers (who were also less motivated to quit) into our intervention, additional research is needed to gain more insight into the underlying factors that foster adherence among LSES smokers. Even though we investigated differences in message processing mechanisms between LSES and HSES smokers, future studies may investigate more in-depth the influence of several affective components, such as user experiences, which may be related to actual use of the intervention (Crutzen et al., 2011). Previous research has already indicated that a positive user experience is related to increased use of the website (Crutzen et al., 2009b). User experience refers to what a person thinks and feels during and after being exposed to a website (Crutzen et al., 2009b). It might be important to study whether LSES and HSES smokers differ in their user perceptions (such as trustworthiness, attractiveness and enjoyment of the intervention) and whether these factors influence actual revisits of the intervention.

Moreover, the short-term results (Chapter 5) suggest that the effects of the intervention depends on adherence, since abstinence rates were overall higher when respondents adhered to more than one session of the intervention. One could argue that adhering to a higher proportion of sessions, results in higher levels of intervention effects. However, too many sessions may, on the other hand, also have reverse effects, especially among LSES smokers who may be exhausted after some time. Therefore, additional research is needed to investigate and determine the optimum dose for a tailored smoking cessation intervention and whether this optimum dose differs between LSES and HSES smokers.

\section{Potential ways to enhance quitting in LSES smokers}

Despite the emerging evidence of the video-based computer-tailored intervention outlined in Chapter 5 and 6, our computer-tailored interventions did not seem to have the burden of increasing inequalities in smoking prevalence, as HSES, MSES and LSES respondents profited equally from the intervention. Continued effort is therefore needed to further optimise and enhance the effectiveness of web-based smoking cessation interventions among LSES smokers in particular. 
The web-based computer-tailored intervention tested in this dissertation was based on a socio-cognitive model in which smoking behaviour is explained by several cognitive factors such as someone's attitude, social norms and perceived self-efficacy towards quitting. Although our approach made it possible to investigate main effects of the video- and textbased intervention among LSES, MSES and HSES smokers, a more in-depth analysis concerning the underlying working mechanisms of the intervention is needed for the refinement of future tailored cessation interventions. To optimise computer-tailored e-health interventions, it is important to know what key components are needed to target LSES smokers (Michie et al., 2009). A detailed analysis of the socio-cognitive key factors used in the two interventions may therefore be desirable to gain better insight into the working mechanisms and to further improve the computer-tailored web-based intervention for LSES smokers who are also often less motivated to quit smoking. In addition, a further exploration of moderating effects for delivery strategy is warranted. This could give us more information regarding what factors should be explicitly targeted to stimulate smoking cessation in LSES groups.

Since our cognitive oriented approach was indicated to be successful in increasing abstinence rates among all socioeconomic groups of smokers, future research may also focus on several affective oriented aspects related to smoking. Previous studies have already indicated that LSES smokers in particular, are often exposed to higher levels of stress and often have fewer affective resources (Albertsen et al., 2003). Furthermore, LSES smokers often perceive smoking as a coping strategy to deal with emotional and stressful situations (Tsourtos and O'Dwyer, 2008). Although respondents during our intervention were stimulated to make plans for emotional situations, future research should aim at investigating specific strategies that could be included in tailored interventions to help LSES smokers to cope with emotional and stressful situations.

Another important step for future research may be to focus more on the smoker's social environment. Although integrated to some extent in our intervention, interventions may profit from also targeting the smoker's social environment (Hiscock et al., 2013). Social support has already been found to be an important factor underlying smoking cessation (Fisher, 1997). Previously, it was indicated that smokers with a lower SES might find quitting more difficult because they often have fewer people in their environment who support their quit attempt (Giskes et al., 2006, Lacey et al., 1993, Lindstrom et al., 2000); this may reduce their chances of quitting. It is therefore plausible that LSES smokers need even more support regarding their social environment. Therefore, additional research is needed to investigate the benefits of a multiple tailored intervention approach targeting both the individual as well as the community. 


\section{Refining motivational routing adapted to smoker's readiness to quit}

We showed that the video-based intervention with the different motivational routings was most effective for smokers with a high (within one month) and low (within four to six month) readiness to quit smoking, but still not adequate for smokers with a moderate (from more than one month to three months) motivation to quit. During our intervention stage-based tailoring was used, which is based on the assumption that smokers in the same stage profit from the same tailored advice (De Vries and Mudde, 1998, Weinstein et al., 1998). In our intervention, smokers intending to quit within a month (preparation) were directed to another motivational routing compared to smokers intending to quit within two to six months (contemplation) (De Vries and Mudde, 1998). Since past research has suggested that the contemplation phase may entail a combination of smokers who are high as well as moderate in their motivation to quit (Herzog, 2008), results were analysed according to three motivational stages (ready to quit within one month, ready to quit within one to three months and ready to quit within four to six months). Results from our effect analyse (Chapter 5) revealed that smokers with a lower motivation to quit profited more from the tailored advice compared to smokers with a moderate motivation to quit. Although these smokers may be categorised in the same stage (according to the I-Change Model), they did not profit equally from the tailored feedback. Future studies might therefore focus on developing and testing tailored feedback that is capable of increasing abstinence rates among smokers with a moderate motivation to quit smoking.

\section{Improve standards of cost-effectiveness studies}

In the cost-effectiveness study presented in this dissertation (Chapter 7), results were interpreted by varying monetary threshold values of smoking abstinence from $€ 0$ up to 80,000. Although a willingness to pay of $€ 18,000$ is an accepted Dutch cut-off point with regard to preventive interventions, to date, no information is available on the societal willingness to pay per abstinent smoker. Although the chosen range of our study from $€ 0$ to $€ 80,000$ might be broad enough to capture all important threshold values, future research should aim to determine a cut-off point for the societal willingness to pay per abstinent respondent in order to choose the most cost-effective treatment, something also suggested by others (Smit et al., 2013). Moreover, future research should also aim to evaluate the QALY measure for smoking populations. Since we were not able to detect short-term health changes in smokers, other measures, which might better capture short-term changes (e.g. satisfaction or enablement), should be considered for inclusion. To enable appropriate interpretation of the results, future studies should also aim to investigate the cost-effectiveness of the intervention among certain subgroup of smokers (smokers with different SES and motivational levels). 


\section{Increasing consensus in analyzing results of a randomized controlled trial}

As was also briefly mentioned earlier, there is inconsistency in previously published papers regarding the analytic approach to test the effectiveness of smoking cessation interventions. There is, for example, still debate about whether the negative scenario approach (treating missing's as smokers) is a too conservative method (Heritier et al., 2003). Although it is a wellestablished approach in smoking-related studies, it could lead to an underestimation of the effects, whereas the complete case scenario might lead to an overestimation of the results (Eysenbach, 2005, Heritier et al., 2003, Hollis and Campbell, 1999). While multiple imputation is regarded as a new preferred method to handle missing data, reliable estimates often depend on the number of missing values (White et al., 2011). Since the result of the multiple imputation technique often depends on the created imputation model (Schulz et al., 2013, Sinharay et al., 2001, White et al., 2011), future research would benefit from investigating different rates of attrition and their influence on the intervention's effectiveness. Although, in the majority of studies in this dissertation, a multiple analytic approach was used to provide a broader perspective, future research should gain more insight into the best analysis approach for smoking-related randomised controlled trials to facilitate interpretation of the intervention effects. Finally, it is also important to obtain more information about the smoking status of respondents who did not return to the follow-up measurements to better interpret the results, as suggested by Elfeddali (Elfeddali et al., 2012a).

\section{Reduction in study-drop out}

To reduce study drop-out in our (cost) effectiveness studies, respondents who did not fill in the six- and 12-month follow-up measurements were reminded after one week by e-mail to complete the web-based questionnaire. A further e-mail reminder was sent after two weeks. Respondents, who did not complete the measurement, received another e-mail inviting them to shortly indicate their current smoking status. This e-mail requested completion of a shortened version of the follow-up measurement, consisting of the 10 most important questions. Finally, when the follow-up measurement was still not completed, respondents were contacted by phone for a short interview, asking the same questions as in the shortened questionnaire. Since drop-out rates in our study were not enormously high compared to those in other e-health studies, about $30 \%$ and $48 \%$ after six and 12 months, future web-based smoking cessation interventions should consider reminding patients by additional reminders or telephone interviews to prevent study drop-out. Additionally, more systematic research may be needed to test strategies to reduce drop-out. 


\section{Implications for improvement of the computer-tailored interventions}

A recommendation concerns the improvement of the computer-tailored smoking cessation intervention. Although we made efforts to ensure that the smoking cessation intervention was adapted to the needs of smokers with different SES and motivational levels, the intervention may benefit from further refinement.

One possibility might be to focus on the preference of delivery strategy. Due to the experimental nature of our study, respondents were not able to choose their favourite delivery strategy (video- vs. text-based messages). A lack of this option may have decreased the overall effectiveness of the intervention. Enabling respondents to choose for their favourite delivery strategy (video- vs. text-based messages) might also improve respondents desire to complete the interventions. Although past research has indicated that respondent's preference regarding delivery mode did not significantly influence the effectiveness of a physical activity intervention (Vandelanotte et al., 2012), it would be useful to examine whether this holds true for our web-based tailored smoking cessation intervention.

Another topic is related to the presenters of the video-based messages. In its current form, five different adults delivered the tailored messages in a TV news program format. To ensure that respondents could identify with the persons delivering the messages, we used a variety of actors with different ages, genders and ethnicities. Yet, another option could be to use several modelling strategies (Hinyard and Kreuter, 2007, Walthouwer et al., 2013). To further increase the credibility of the tailored advice, role models could be included with which respondents can identify. Role models could explain how they struggled with their own quit attempt and how they succeeded in achieving their goal (Walthouwer et al., 2013). Additionally, future interventions might profit from recording the video message, illustrating specific at risk situations for relapse (such as at a party) to increase authenticity of the videobased messages and to provide more detailed feedback. Research investigating under which circumstances and for whom different role models are preferred might lead to an enhanced effect of the intervention.

Next, drop-out rates were higher among respondents in the video-based intervention (Chapter 5). To boost participation in video-based smoking cessation interventions, tailored messages might need to be shortened. Past research in another health-related domain has already indicated that respondents want the messages to be shorter (Vandelanotte and Mummery, 2011). Although previous research has shown that higher exposure rates have led to higher intervention effectiveness (Elfeddali et al., 2012a, Kreuter et al., 1999), a potential way to improve participation and adherence in these interventions may be to shorten the duration of the video messages.

Furthermore, the content of the two interventions was exactly the same to test the added effect of one of the two delivery strategies. It is possible that the experimental conditions (video- vs. text-based messages) may have been offered in a more engaging way. 
To further improve the intervention, elements such as graphics, cartoons and other animations effects could be included.

Additionally, the intervention probably may not have included enough social support strategies to increase smoking cessation among LSES smokers. Past research has already shown that couple-based smoking cessation interventions were effective in increasing smoking abstinence rates among smokers (Lee and Kahende, 2007, Shoham et al., 2006). To enhance the effectiveness of the intervention especially among LSES smokers, the intervention could further integrate strategies to increase support from their social environment, this could be done by involving respondent's social environment (e.g. friends, partners etc.) in the intervention.

During our intervention, respondents received tailored feedback at fixed time points and were not able to receive support at other times when they might need it the most. As previously indicated by others (Smit et al., 2012), smoking cessation interventions targeted to the personal needs of respondents (such as receiving feedback at individual time-moments) may be more effective in increasing smoking abstinence rates. Smokers and especially LSES smokers may need more flexible programs, targeted to their personal wishes at times when they need support. To increase participation and quit rates in these interventions, ideally the number and duration of the different sessions should be adjusted in response to the wishes of LSES respondents. Consequently, ecological momentary assessment strategies, which are able to monitor fluctuations in smoking behaviour, might be fruitful to incorporate in future smoking cessation interventions (Heron and Smyth, 2010) in order to increase participation and engagement among LSES smokers.

\section{General conclusion}

Based on the different studies presented in the current dissertation, several conclusions can be drawn with regard to the computer-tailored smoking cessation intervention. First, mode of recruitment influenced the type of smoker that participated in the computer-tailored intervention. While recruitment via GPs resulted in a higher percentage of LSES smokers who quit smoking, recruitment via mass media approaches (such as Internet and newspaper advertising) resulted in a larger absolute number of smokers who made a quit attempt. Second, although delivery strategy (video-based vs. text-based messages) seemed to play no role in the processing of the tailored information, LSES smokers showed higher attention and processing levels of the tailored messages and were more inclined to visit the computer-tailored intervention again compared to HSES smokers. Third, the video-based computer-tailored intervention was especially effective in increasing short-term abstinence compared to the control condition in smokers with a lower motivation to quit smoking, on the most valid outcome measurement, namely prolonged abstinence. Furthermore, the video-based 
computer-tailored intervention was effective for achieving long-term smoking abstinence. No differential effects were found for LSES, MSES and HSES smokers. Fourth, the video-based computer-tailored intervention was also the most cost-effective treatment for smoking abstinence, when varying the willingness to pay per abstinent respondent from $€ 0$ up to $€ 80,000$. Since the video-based intervention showed more effects and lower costs, we advise to implement video-based messages above text-based messages. Fifth, even though the videobased intervention was successful in increasing smoking abstinence rates, the intervention may benefit from further improvement to optimise its potential. 
Valorisation addendum

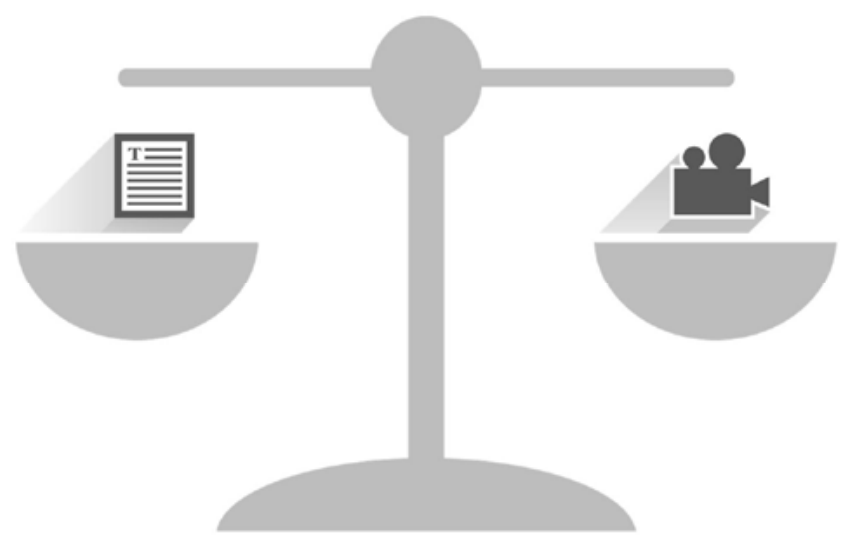





\section{Relevance}

Smoking can lead to several illnesses not only in smokers but also in non-smokers who are exposed to second hand smoke (World Health Organization, 2011). The resulting smokingrelated diseases affect the individual and their families, but also put an economic burden on society as they increase overall health-care costs (Parrott and Godfrey, 2004). The costs of smoking to society include not only health-care-related costs but also costs related to productivity loss due to morbidity (Barendregt et al., 1997, Welte et al., 2000). Society pays for the costs of smoking in the form of higher health insurance, medical related costs, higher taxes and higher prices for health-care products and services. Even if smokers, on average, die earlier than people who have never smoked, savings as a result of earlier morbidity among smokers in terms of medical expenditures often do not counterweight their overall higher medical costs (Hodgson, 1998, Rasmussen et al., 2004). Furthermore, lifetime work productivity is often higher among non-smokers compared to smokers. Although smoking increases the risk of illness and premature death among all consumers, tobacco use is greatest in groups with a lower socioeconomic status (LSES) (Giskes et al., 2005, Kotz and West, 2009). Compared to smokers with higher socioeconomic status (HSES), LSES smokers are more often daily smokers, they often have a lower motivation to quit, show fewer attempts to quit and more often relapse to smoking. Consequently, the reduction of tobacco use and smoking cessation in LSES groups is necessary to reduce health-care-related costs in the future and can be seen as a major benefit for society.

Both the six- and 12-month effects of the randomised controlled trial, as well as the cost-effectiveness study of this dissertation, demonstrated that a video-based computertailored intervention can be (cost) effective in achieving smoking abstinence in smokers, independent of the SES status of the smoker. Therefore, implementation of this video-based intervention is recommended in order to contribute to a reduction of the number of smokers among different SES groups in the Netherlands.

\section{Target groups}

The results presented in this thesis are of interest for several sectors of society. First, the results might be of interest to policy makers of the Dutch Ministry of Health, Welfare and Sport. One of the primary aims of the Dutch government's IT policy for the health-care sector is to improve affordability, access and quality of health care. Decisions in the public sector are more driven by cost-effective treatments. Given the fact that policy makers and health-care budget holders have to make choices regarding the implementation of different smoking cessation programmes, it is essential to know whether the societal benefits of these smoking cessation programmes are worth the investments that have to be made to offer them (Park et al., 2013). Especially in times of rising health-care costs and limited resources, the results might guide 
policy makers who need to make choices regarding the implementation of several smoking cessation programmes.

Second, another highly relevant task group consists of Dutch health care organisations such as the Dutch Cancer Society, the Dutch Asthma Foundation, the Dutch Heart Foundation, the Diabetes Fund, the Dutch Association for Residential and Home Care, the Centre of Expertise on Mental Health and Addiction and the Netherlands Smokefree Alliance. As our web-based smoking cessation intervention has shown to be effective as well as cost-effective, they might use this information for the delivery of tailored smoking advice to their customers. With the current intervention, they could complement their current offer of programmes with an evidence- and web-based program that is also cost-effective.

Third, the insights could also be useful for the Dutch College of General Practitioners (GPs) who support evidence-based general practice. The GP setting can be considered an important access point to reach smokers and LSES citizens in particular. In the Netherlands, smoking cessation treatment recommendations (which are described in the guideline of the 'Dutch College of GPs') are to ask patients with smoking-related illnesses about their smoking behaviour, to give advice on how to stop smoking and to refer the patient to stop smoking treatment programmes (Chavannes et al., 2007). However, GPs, practice nurses and other health professionals often indicate a lack of time and skills to provide their smoking patients with smoking cessation advice. As the workload of Dutch health professionals and GPs remains high, the integration into health care practice of an effective web-based tailored smoking cessation program that does not require a great amount of time - as our web-based smoking cessation intervention - may lower the burden of work for the GPs and practice nurses.

Fourth, the insights of the results might also be of interest for the Dutch Association of E-health, which contributes to the development of policy and standards in e-health on behalf of health professionals and patients. They offer a platform to exchange knowledge, skills and research outcomes and collaborate with professionals to improve the quality of e-health services.

Finally, the results might be of interest for the Centre for Healthy Living (Centrum Gezond Leven (in Dutch)). The Centre supports the delivery of efficient and effective local health promotion in the Netherlands and presents current information on the quality, efficiency and coherence of health promotion interventions.

\section{Activities and products}

The results of the present dissertation have documented that a video-based, computertailored smoking cessation intervention is successful as well as cost-effective. This means that it is recommended to implement this effective video-based smoking-cessation intervention nationwide among the Dutch population. Smokers should receive a brief advice to stop 
smoking by GP's. If appropriate, the smoker could be advised to our smoking cessation intervention. Smokers will receive personalised feedback on their smoking behaviour, their attitude, perceived social influence, self-efficacy and how to prepare to quit. The intervention is effective and suitable for all smokers, independent of their motivation and intention to quit. Therefore, the intervention is also effective for those who are less motivated to quit smoking. Smokers who will set their quit date within a month will be helped to translate their intention to quit smoking into action. Smokers who are not ready to quit smoking will receive feedback to increase their motivation by increasing the perception of the pros of quitting and knowledge of how to obtain support for quitting.

\section{Innovation}

Most of today's computer-tailored smoking cessation approaches often rely on text-based messages. Previous research has suggested that health communication probably needs a different approach with less cognitive elaboration and the utilisation of videos, especially in LSES smokers (Ma et al., 2005, Mayer and Massa, 2003). To our knowledge, computer-tailored interventions do not yet incorporate the potential of video-based messages in their communication strategy to better reach LSES smokers. Therefore, the aim of this dissertation was to investigate the influence of delivery strategy (video vs. text-based messages) towards a computer-tailored smoking cessation intervention among smokers with different SES and motivational quitting levels. This was one of the first research projects that has tested the effects of video information in the context of smoking cessation among different socioeconomic groups.

Results of the current dissertation provide important new evidence for the effectiveness of video-tailored information towards smoking abstinence in both LSES and HSES smokers and smokers unmotivated to quit. As video content has shown to improve the appeal of our smoking cessation intervention, the integration of video messages in future smoking cessation programs might be a promising new strategy to attract and engage smokers of different SES levels.

\section{Schedule and implementation}

Given the encouraging results of the video-based, computer-tailored intervention, nationwide implementation is recommended.

An important first step is to certify the web-based intervention at the Centre for Healthy Living. The Centre has already certified an earlier version of the web-based intervention for Healthy Living as an effective and evidence-based intervention. Currently, plans for certification have been developed for this program as well. The current intervention - as it is a standalone intervention - can then be implemented in multiple settings. One setting 
would be the GP setting. As mentioned earlier, the GP setting represents an important opportunity to reach LSES smokers. Yet, in practice, time limits the amount of attention GPS and their practice nurses can give their smoking patients. The implementation of a web-based intervention that does not require an extensive amount of time from the GP or practice nurse might, therefore, be beneficial. Another possibility that does not preclude the former is that the intervention will be implemented by several Dutch health care organisations (such as the Centre of Expertise on Mental Health and Addiction and the Netherlands Smokefree Alliance) that are able to spread the intervention among the Dutch smoking population and who may reach a greater number of smokers. Moreover, the intervention could also be adopted and disseminated by several regional public health authorities (in Dutch GGD) that provide an important access point for reaching a large segment of the Dutch population.

Yet, in today's cost-conscious environment, there are often limited resources available to guarantee adoption of effective interventions. Main barriers to implementing such interventions include lack of funding, incentives, time of health professionals, knowledge concerning effective programmes, support from organisations and repetitive other new programmes (Fleuren et al., 2004). Several steps might be required for a successful implementation. As already formulated by the Diffusion of Innovation Theory, implementing an intervention is a process that occurs over time and consists of multiple phases: knowledge, persuasion, decision, implementation and confirmation (Rogers, 2003). The formation of a multidisciplinary team that develops an implementation plan (consisting of policy makers, academic researchers, health professionals and leaders of Dutch health funds) could be an important first step for implementation of the intervention in the Dutch smoking population to ensure its integration and support at different levels. The next step is to encourage health organisations and health professionals to deliver the intervention. The expected benefits of the program need to be promoted in order to encourage health care workers to use the cessation intervention in their daily practice. Besides, guidelines need to be developed that explain the cessation intervention and how it should be delivered and promoted by health care workers. Finally, when the program is implemented, it is also important to ensure that the intervention will be monitored and evaluated over time to ensure that barriers and problems are being addressed and resolved. 


\section{REFERENCES}

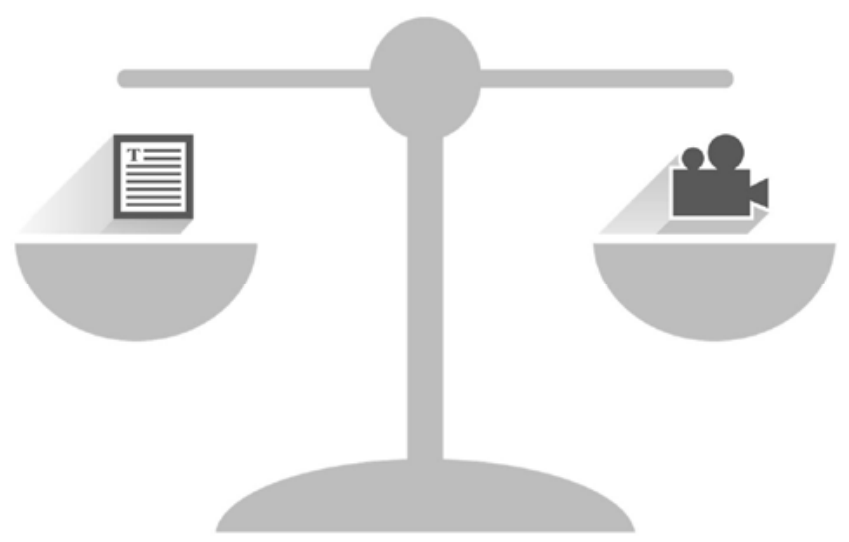



Abrams, D. B., Orleans, C. T., Niaura, R. S., Goldstein, M. G., Prochaska, J. O. \& Velicer, W. (1996). Integrating individual and public health perspectives for treatment of tobacco dependence under managed health care: a combined stepped-care and matching model. Annals of Behavioral Medicine: a publication of the Society of Behavioral Medicine, 18, 290-304.

Acock, A. C. (2005). Working with missing values. Corvallis, Oregon State University.

Ajzen, I. (1985). From intentions to actions: A theory of Planned Behaviour. Heidelberg, Springer.

Albertsen, K., Hannerz, H., Borg, V. \& Burr, H. (2003). The effect of work environment and heavy smoking on the social inequalities in smoking cessation. Public Health, 117, 383-388.

Alexander, G. L., McClure, J. B., Calvi, J. H., Divine, G. W., Stopponi, M. A., Rolnick, S. J., Heimendinger, J., Tolsma, D. D., Resnicow, K., Campbell, M. K., Strecher, V. J. \& Johnson, C. C. (2010). A randomized clinical trial evaluating online interventions to improve fruit and vegetable consumption. American Journal of Public Health, 100, 319-326.

American Psychological Association. (2002). Ethical Principles of Psychologists and Code of Conduct. American Psychologist, 57, 1060-1073.

Armitage, C. J. \& Conner, M. (2001). Efficacy of the Theory of Planned Behaviour: a meta-analytic review. British Journal of Social Psychology, 40, 471-499.

Ausems, M., Mesters, I., van Breukelen, G. \& de Vries, H. (2002). Short-term effects of a randomized computerbased out of school smoking prevention trial aimed at elementary school children. Preventive Medicine, 34, 581-589.

B

Bailenson, J. N., Blascovich, J. \& Guadagno, R. E. (2008). Self-representations in immersive virtual environment. Journal of Applied Social Psychology, 38, 2673-2690.

Balmford, J., Borland, R., \& Benda, P. (2008). Patterns of use of an automated interactive personalized coaching program for smoking cessation. Journal of Medical Internet Research, 10, e54.

Balmford, J., Borland, R., Benda, P. \& Howard, S. (2013). Factors associated with use of automated smoking cessation interventions: findings from the eQuit study. Health Education Research, 28, 288-299.

Bandura, A. (1986). Social foundations of thought and action: a social cognitive theory. Engewood Cliff, New York, Prentice-Hall.

Barendregt, J. J., Bonneux, L. \& van der Maas, P. J. (1997). The health care costs of smoking. The New England Journal of Medicine, 337, 1052-1056. 
Bauld, L., Bell, K., McCullough, L., Richardson, L. \& Greaves, L. (2010). The effectiveness of NHS smoking cessation services: A systematic review. Journal of Public Health, 32, 71-82.

Bauld, L., Judge, K. \& Platt, S. (2007). Assessing the impact of smoking cessation services on reducing health inequalities in England: observational study. Tobacco Control, 16, 400-404.

Baylor A. L. (2009). Promoting motivation with virtual agents and avaters: role of visual presence and appearance. Philosophical Transactions of the Royal Society, 364, 3559-3565.

Belisario, J. S. M., Bruggeling, M. N., Gunn, L. H., Brusamento, S. \& Car, J. (2012). Interventions for recruiting smokers into cessation programmes. Cochrane Database of Systematic Reviews, CD009187.

Bell, K., McCullough, L., Greaves, L., Mulryne, R., Jategaonkar, N. \& de Vries, K. (2006). The Effectiveness of National Health Service Intensive treatments for Smoking Cessation in England: a rapid review. London, UK, National Institute for Clinical and Health Excellence.

Bennett, G. G. \& Glasgow, R. E. (2009). The delivery of public health interventions via the Internet: actualizing their potential. Annual Review of Public Health, 30, 273-292.

Berndt, N., Bolman, C., Froelicher, E. S., Mudde, A., Candel, M., de Vries, H. \& Lechner, L. (2013). Effectiveness of a telephone delivered and a face-to-face delivered counseling intervention for smoking cessation in patients with coronary heart disease: a 6-month follow-up. Journal of Behavioral Medicine, 1-16.

Blankers, M., Koeter, M. W. \& Schippers, G. M. (2010). Missing data approaches in e-health research: simulation study and a tutorial for nonmathematically inclined researchers. Journal of Medical Internet Research, 12 , e54.

Bodner, T. E. (2008). What improves with increased missing data imputations? Structural Equation Modeling. A Multidisciplinary Journal, 14, 651-675.

Bolman, C. \& de Vries, H. (1998). Psycho-social determinants and motivational phases in smoking behaviour of cardiac inpatients. Heart and Lung, 31, 15-24.

Borland, R., Balmford, J. \& Hunt, D. (2004). The effectiveness of personally tailored computer-tailored advice letters for smoking cessation. Addiction, 99, 369-377.

Bouwens J., Kleinjan, M., Peters, L. \& Eijnden, R. (2007). Aanbevelingenonderzoek en implementatie op het terrein van de aanpak van roken [Online]. Woerden/Rotterdam, Nigz/IVO. Available from: http:// www.nigz.nl/index.cfm?act=winkel.detail\&pid=912\&ShoplD=.

Brouwer, W., Kroeze, W., Crutzen, R., de Nooijer, J., de Vries, N. K., Brug, J. \& Oenema, A. (2011). Which intervention characteristics are related to more exposure to internet-delivered healthy lifestyle promotion interventions? A systematic review. Journal of Medical Internet Research, 13, e2. 
Brouwer, W., Oenema, A., Raat, H., Crutzen, R., de Nooijer, J., de Vries, N. K. \& Brug, J. (2010). Characteristics of visitors and revisitors to an Internet-delivered computer-tailored lifestyle intervention implemented for use by the general public. Health Education Research, 25, 585-595.

Brouwner, C., Preloran, M. \& Press, N. (1996). The effects of ethinicity, education and an informational video on pregnant women's knowledge and decisions about a prenatal diagnostic screening test. Patient Education and Counseling, 27, 135-146.

Brug, J., Campbell, M. \& van Assema, P. (1999). The application and impact of computer-generated personalized nutrition education: a review of the literature. Patient Education and Counseling, 36, 145-156.

Brug, J., Oenema, A. \& Campbell, M. (2003). Past, present, and future of computer tailored nutrition education. American Journal of Clinical Nutrition, 77, 1028-1034.

Businelle, M. S., Kendzor, D. E., Reitzel, L. R., Costello, T. J., Cofta-Woerpel, L., Li, Y., Mazas, C. A., Vidrine, J. L., Cinciripini, P. M., Greisinger, A. J. \& Wetter, D. W. (2010). Mechanisms linking socioeconomic status to smoking cessation: a structural equation modeling approach. Health Psychology, 29, 262-273.

C

Cacioppo, J., Petty, R., Feinstein, J. \& Jarvis, W. B. G. (1996). Dispositional differences in cognitive motivation: The life and times of individuals varying in need for cognition. Psychological Bulletin, 119, 197-253.

Cacioppo, J. T. \& Petty, R. E. (1982). The need for cognition. Journal of Personality and Social Psychology, 42, 116131.

Calvert, S. L., Rideout, V. J., Woolard, J. L., Barr, R. F. \& Strouse, G. A. (2005). Age, ethnicity, and socioeconomic patterns in early computer use - A national survey. American Behavioral Scientist, 48, 590-607.

Campbell, M., DeVellis, B., Strecher, V. J., Ammerman, A. S., DeVellis, R. F. \& Sandler, R. S. (1994). Improving dietary behaviour: the effectiveness of tailored messages in primary care settings. American Journal of Public Health, 84, 783-787.

Campbell, M. K., Honess-Morreale, L., Farrell, D., Carbone, E. \& Brasure, M. (1999). A tailored multimedia nutrition education pilot program for low-income women receiving food assistance. Health Education Research, 14, 257-267.

Casparie, A. F., van Hout, B. A. \& Simoons, M. L. (1998). Guidelines and costs. Nederlands Tijdschrift voor Geneeskunde, 142, 2075-2077.

Cavelaars, A. E., Kunst, A. E., Geurts, J. J., Crialesi, R., Grotvedt, L., Helmert, U., Lahelma, E., Lundberg, O., Matheson, J., Mielck, A., Rasmussen, N., Regidor, E., Rosário-Giraldes, M., Spuhler, T. \& Mackenbach, J. P. (2000). Educational differences in smoking: international comparison. British Medical Journal, 320, 1102-1107. 
CBS. (2011a). ICT gebruik van personen naar persoonskenmerken [Online]. Heerlen, Dutch Central Institute for statistics. Avalaible from: http://statline.cbs.nl/StatWeb/publication/?VW=T\&DM=SLNL\&PA=71098 ned\&D1=33,55-59,93-95,97-100,102-104,106-109,112-113,119,124-133\&D2=0\&D3=0,I\&HD=130422$1124 \& H D R=G 2, G 1 \& S T B=T$.

CBS. (2011b). CBS/MOA GS definitie[Online]. Heerlen, Dutch Central Institute for statistics. Available from: http://www.google.nl/url?sa=t\&rct=j\&q=\&esrc=s\&frm=1\&source=web\&cd=1\&ved=0CDQQFjAA\&url=ht tp\%3A\%2F\%2Fwww.moaweb.nl\%2FServices\%2Fgouden-standaard\%2Fpresentaties-goudenstand aard\%2FMOA\%2520GS-Constructen-afgeleiden.pdf\%2Fat_download\%2Ffile\&ei=NTEgU8mvJ6iq0QW VsYH gCw\&usg=AFQjCNGuaoJl6zZieNhj2qUsfgA5dDZT1g.

CBS. (2012). Opleidingniveaus van de standard onderwijsindeling [Online]. Heerlen, Dutch Central Institute for statistics. Avalaible from: http://www.cbs.nl/NR/rdonlyres/7C94DE33-621C-4355-928A-8B90F9F5 D777/0/2006soiniveauindeling201213.pdf.

CBS. (2013). Overview of consumer price indexes [Online]. Heerlen, Dutch Central Institute for statistics. Avalaible from: http://statline.cbs.nl/statweb/.

CDC. (2004). The health consequences of smoking: a report of the Surgeon General. Washington D. C., Centers for Disease Control and Prevention.

Chalkidou, K., Culyer, A., Naidoo, B. \& Littlejohns, P. (2008). Cost-effective public health guidance: Asking questions from the decision-maker's viewpoint. Health Economics, 17, 441-448.

Chan, S. S., Leung, D. Y., Abdullah, A. S., Wong, V. T., Hedley, A. J. \& Lam, T. H. (2011). A randomized controlled trial of a smoking reduction plus nicotine replacement therapy intervention for smokers not willing to quit smoking. Addiction, 106, 1155-1163.

Chandola, T., Head, J. \& Bartley, M. (2004). Socio-demographic predictors of quitting smoking: how important are household factors? Addiction, 99, 770-777.

Chavannes, N. H., Kaper, J., Frijling, B. D., van der Laan, J. R., Jansen, P. W. M., Guerrouj, S., Drenthen, A. J. M., Bax, W. \& Wind, L. A. (2007). NHG-Standaard Stoppen met roken. Huisarts Wet, 50, 306-314.

Chen, Y. F., Madan, J., Welton, N., Yahaya, I., Aveyard, P., Bauld, L., Wang, D., Fry-Smith, A. \& Munafo, M. R. (2012). Effectiveness and cost-effectiveness of computer and other electronic aids for smoking cessation: a systematic review and network meta-analysis. Health Technology Assessment, 16, 1-205.

Civljak, M., Sheikh, A., Stead, L. F. \& Car, J. (2010). Internet-based interventions for smoking cessation. Cochrane Database of Systematic Reviews, CD007078.

Civljak, M., Stead, L. F., Hartmann-Boyce, J., Sheikh, A. \& Car, J. (2013). Internet-based interventions for smoking cessation. Cochrane Database of Systematic Reviews, 7, CD007078. 
Cohen, A. L., Bayer, U. C., Jaudas, A. \& Gollwitzer, P. M. (2008). Self-regulatory strategy and executive control: implementation intentions modulate task switching and Simon task performance. Psychological Research, 72, 12-26.

Cole, J. C., Rabin, A. S., Smith, T. L. \& Kaufman, A. S. (2004). Development and validation of a Rasch-derived CESD short form. Psychological Assessment, 16, 360-372.

Connor Gorber, S., Schofield-Hurwitz, S., Hardt, J., Levasseur, G. \& Tremblay, M. (2009). The accuracy of selfreported smoking: a systematic review of the relationship between self-reported and cotinine-assessed smoking status. Nicotine Tobacco Research, 11, 12-24.

Crutzen, R. (2009a). Hard to get, hard to keep: Dissemination of and exposure to Internet-delivered Health behaviour change interventions aimed at adolescents (Doctoral Thesis), Maastricht University, Maastricht.

Crutzen, R., Cyr, D. \& de Vries, N. K. (2011). Bringing loyalty to e-health: Theory validation using three Internetdelivered interventions. Journal of Medical Internet Research, 13, e73.

Crutzen, R., de Nooijer, J., Brouwer, W., Oenema, A., Brug, J. \& de Vries, N. K. (2009b). A conceptual framework for understanding and improving adolescents' exposure to Internet-delivered interventions. Health Promotion International, 24, 277-284.

Crutzen, R., de Nooijer, J., Brouwer, W., Oenema, A., Brug, J. \& de Vries, N. K. (2009c). Effectiveness of online word of mouth on exposure to an Internet-delivered intervention. Psychology and Health, 24, 651-661.

Crutzen, R., Peters, G. J. Y., \& Abraham, C. (2012). Data sharing among trialists. What about trialists sharing other study materials? British Medical Journal, 345, e8352.

Cyr, D., Bonanni, C., Bowes. J. \& Ilsever, J. (2005). Beyond Trust: website design preferences across cultures. Journal of Global Information Management, 13, 24-52.

Cyr, D., Head, M. \& Ivanov, A. (2009). Perceived interactivity leading to e-loyalty: development of a model for cognitive-affective user responses. International Journal of Human-Computer Studies, 67, 850-869.

Czarnecki, K. D., Vichinsky, L. E., Ellis, J. A. \& Perl, S. B. (2010). Media campaign effectiveness in promoting a smoking-cessation program. American Journal of Preventive Medicine, 38, 333-342.

D

Dalstra, J. A., Kunst, A. E., Geurts, J. J., Frenken, F. J. \& Mackenbach, J. P. (2002). Trends in socioeconomic health inequalities in the Netherlands, 1981-1999. Journal of Epidemiology and Community Health, 56, 927934.

Danaher, B. G., Jazdzewski, S. A., McKay, H. G. \& Hudson, C. R. (2005). Bandwidth constraints to using video and other rich media in behavior change websites. Journal of Medical Internet Research, 7, e49. 
De Nooier, J., Oenema, A., Kloek, G., Brug, H., de Vries, H. \& de Vries, N. (2005). Bevordering van gezond gedrag via het Internet: Nu en in de toekomst [Promotion of healthy behavior through the Internet: now and in the future]. Maastricht, Universiteit Maastricht.

De Vries H. \& Brug H. (1999). Computer tailored interventions motivating people to adopt health promoting behaviours: introduction to a new approach. Patient Education and Counseling, 36, 99-105.

De Vries, H., Eggers, S. M. \& Bolman, C. (2013). The role of action planning and plan enactment for smoking cessation. BMC Public Health, 13, 393.

De Vries, H., Kremers, S. P. J., Smeets, T., Brug, J. \& Eijmael, K. (2008). The effectiveness of tailored feedback and action plans in an intervention addressing multiple health behaviors. American Journal of Health Promotion, 22, 417-425.

De Vries, H., Mudde, A., Leijs, I., Charlton, A., Vartiainen, E., Buijs, G., Clemente, M. P., Storm, H., GonzálezNavarro, A., Nebot, M., Prins, T. \& Kremers, S. (2003). The European smoking prevention framework approach (EFSA): an example of integral prevention. Health Education Research, 18, 611-626.

De Vries, H. \& Mudde, A. N. (1998). Predicting stage transitions for smoking cessation applying the attitude-social influence-efficacy model. Psychology and Health, 13, 369-385.

Dijkstra, A. (2005). Working mechanims of computer-tailored health education: evidence from smoking cessation. Health Education Research, 20, 527-539.

Dijkstra A. \& de Vries, H. (1999). The development of computer-generated tailored interventions. Patient Education and Counseling, 36, 193-203.

Dijkstra, A. \& de Vries, H. (2000a). Clusters of precontemplating smokers defined by the perception of the pros, cons, and self-efficacy. Addictive Behaviors, 25, 373-385.

Dijkstra, A. \& de Vries, H. (2000b). Self-efficacy with regard to different tasks in smoking cessation. Psychology and Health, 14, 501-511.

Dijkstra, A., de Vries, H. \& Bakker, M. (1996). Pros and cons of quitting, self-efficacy, and the stages related to smoking cessation. Journal of Consulting and Clinical Psychology, 64, 758-763.

Dijkstra, A., de Vries, H. \& Roijackers, J. (1998a). Long-term effectiveness of computer-generated tailored feedback in smoking cessation. Health Education Research, 13, 207-214.

Dijkstra, A., de Vries, H., Roijackers, J. \& van Breukelen, G. (1998b). Tailoring information to enhance quitting in smokers with low motivation to quit: three basic efficacy questions. Health Psychology, 17, 513-531.

Dijkstra, A., de Vries, H. \& Roijackers, M. A. (1999). Targeting smokers with low readiness to change with tailored and nontailored self-help materials. Preventive Medicine, 28, 203-211. 
Dotinga, A., Schrijvers, C. T., Voorham, A. J. \& Mackenbach, J. P. (2005). Correlates of stages of change of smoking among inhabitants of deprived neighbourhoods. European Journal of Public Health, 15, 152-159.

Drummond, M. (2001). Introducing economic and quality of life measurements into clinical studies. Annals of Medicine, 33, 344-349.

Drummond, M. F., Sculpher, M. J. \& Thorrance, G. W. (2005). Methods for the economic evaluation of health care programmes, New York, Oxford University Press.

Dutch Trial Register. (2013). Dutch Trial Register [Online]. Available from: http://www.trialregister.nl/trial reg/admin/rctview.asp?TC=3102.

$E$

Echer, I. C. \& Barreto, S. S. (2008). Determination and support as successful factors for smoking cessation. Revista Latino-Americana de Enfermagem, 16, 445-451.

Elfeddali, I., Bolman, C., Candel, M. J., Wiers, R. W. \& de Vries, H. (2012a). Preventing smoking relapse via Webbased computer-tailored feedback: a randomized controlled trial. Journal of Medical Internet Research, 14, e109.

Elfeddali, I., Bolman, C., Candel, M. J. J. M., Wiers, R. W. \& de Vries, H. (2012b). The role of self-efficacy, recovery self-efficacy, and preparatory planning in predicting short-term smoking relapse. British Journal of Health Psychology, 17, 185-201.

Elfeddali, I., Bolman, C. \& de Vries, H. (2012c). SQ4U- a computer tailored smoking relapse prevention program incorporating planning strategies and multiple feedback time points after the quit-attempt: development and design protocol. Contemporary Clinical Trials, 33, 151-158.

Ellaway, A. \& Macintyre, S. (2009). Are perceid neighbourhood problems associated with the likelihood of smoking? Journal of Epidemiology and Community Health, 63, 78-80.

Etter, J. F. (2005). Comparing the efficacy of two Internet-based computer-tailored smoking cessation programs: A randomized trial. Journal of Medical Internet Research, 8, e2.

EuroQol - a new facility for the measurement of health-related quality of life. (1990). Health Policy, 16, $199-208$.

Evers, K. E., Cummins, C. O., Prochaska, J. O. \& Prochaska, J. M. (2005). Online health behaviour and diseases management programs: are we ready for them? Are they ready for us? Journal of Medical Internet Research, 7, e27.

Evers, S., Wolfs, C. \& Van Heugten, C. (2010). Economische evaluatie van neuropsychologische behandeling. Amsterdam, Boom Uitgeverij.

Eysenbach, G. (2005). The law of attrition. Journal of Medical Internet Research, 7, e11. 
Ezzati, M. \& Lopez, A. D. (2003). Estimates of global mortality attributable to smoking in 2000. Lancet, 362, 847852 .

F

Fagerström, K. (2012). Determinants of tobacco use and renaming the FTND to the Fagerström Test for Cigarette Dependence. Nicotine Tobacco Research, 14, 75-78.

Feenstra, T. L., Hamberg-van Reenen, H. H., Hoogenveen, R. T. \& Rutten-van Molken, M. P. (2005). Costeffectiveness of face-to-face smoking cessation interventions: a dynamic modeling study. Value in health: the Journal of the International Society for Pharmacoeconomics and Outcomes Research, 8, 178-190.

Fidler, J. A., Jarvis, M. J., Mindell, J. \& West, R. (2008) Nicotine intake in cigarette smokers in England: Distribution and demographic correlates. Cancer Epidemiology, Biomakers and Prevention, 17, 3331-3336.

Fiore, M. C., Jaen, C. R., Baker, T. B., Bailey, W. C., Benowitz, N., Curry, S. J., Dorfman, S. F., Fraser, D., Froehlicher, E. S., Goldstein, M. G., Hasselblad, V., Healton, C. G., Heishman, R. B., Huston, C., Koh, H. K., Kottke, T. E., Lando, H. A., Leitzke, C., Mecklenburg, R. E., Mermelstein, R. J., Morgan, G., Mullen, P. D., Murray, E. W., Orleans, C. T., Piper, M. E., Robinson, L., Stitzer, M. L., Theobald, W., Tommasello, A. C., Villejo, L., Webers, M. E. \& Williams, C. (2008). Clinical practice guideline: Treating tobacco use and dependence. Public Health Service, 35, 158-176.

Fishbein, M. \& Ajzen, I. (1975). Belief, Attitude, Intention and Behaviour: An introduction to theory and research. Reading, MA, Addison-Wesley.

Fishbein M, \& Ajzen I. (2010). Predicting and Changing behaviour: The Reasoned Action Approach. New York, Psychology Press, Taylor and Francis.

Fisher, E. B. (1997). Two approaches to social support in smoking cessation: commodity model and nondirective support. Addiction Behavior, 22, 819-833.

Fisher, E. B., Auslander, W. F., Munro, J. F., Arfken, C. L., Brownson, R. C. \& Owens, N. W. (1998). Neighbors for a smoke free north side: Evaluation of a community organization approach to promoting smoking cessation among African Americans. American Journal of Public Health, 88, 1658-1663.

Fleuren, M., Wiefferink K. \& Paulussen, T. (2004). Determinants of innovation within health care organizations. International Journal for Quality in Health Care, 16, 107-123.

Flycatcher Internet Research. (2012). Het onderzoeksbureau voor online onderzoek [Online]. Available from: http://www.flycatcher.eu.

Frenn, M., Malin, S., Brown, R. L., Greer, Y., Fox, J., Greer, J. \& Smyczek, S. (2005). Changing the tide: An Internet/video exercise and low-fat diet intervention with middle-school students. Applied Nursing Research, 18, 13-21. 
Gallo, L. C. \& Matthews, K. A. (2003). Understanding the association between socioeconomic status and physical health: Do negative emotions play a role? Psychological Bulletin, 129, 10-51.

Gerhards, S. A., de Graaf, L. E., Jacobs, L. E., Severens, J. L., Huibers, M. J., Arntz, A., Riper, H., Widdershoven, G., Metsemakers, J. F. \&Evers, S. M. (2010). Economic evaluation of online computerised cognitivebehavioural therapy without support for depression in primary care: Randomised trial. The British Journal of Psychiatry, 196, 310-318.

Giskes, K., Kunst, A. E., Ariza, C., Benach, J., Borrell, C., Helmert, U., Judge, K., Lahelma, E., Moussa, K., Ostergren, P. O., Patja, K., Platt, S., Prättälä, R., Willemsen, M. C. \& Mackenbach, J. P. (2007). Applying an equity lens to tobacco-control policies and their uptake in six Western-European countries. Journal of Public Health Policy, 28, 261-280.

Giskes, K., Kunst, A. E., Benach, J., Borrell, C., Costa, G., Dahl, E., Dalstra, J. A., Fedrico, B., Helmert, U., Judge, K., Lahelma, E., Moussa, K., Ostergren, P. O., Platt, S., Prattala, R., Rasmussen, N. K. \& Mackenbach, J. P. (2005). Trends in smoking behaviour between 1985 and 2000 in nine European countries by education. Journal of Epidemiology and Community Health, 59, 395-401.

Giskes, K., van Lenthe, F. J., Turrell, G., Brug, J. \& Mackenbach, J. P. (2006). Smokers living in deprived areas are less likely to quit: A longitudinal follow-up. Tobacco Control, 15, 485-488.

Glasgow, R. E. (2007). E-health evaluation and dissemination research. American Journal of Preventive Medicine, $32,119-126$.

Glasgow, R. E., Vogt, T. M. \& Boles, S. M. (1999). Evaluating the public health impact of health promotion interventions: The RE-AIM framework. American Journal of Public Health, 89, 1322-1327.

Glick H. A., Doshi, J. A., Sonnad, S. S. \& Polsky, D. (2007). Economic evaluation in clinical trials. Oxford, Oxford University Press.

Gold, M. (1996). Panel on cost-effectiveness in health and medicine. Medical Care, 34, 197-199.

Gollwitzer, P. (1999). Implementation intentions. Strong effects of simple plans. American Psychologist, 54, 439503.

Graham, A. L., Milner, P., Saul, J. E. \& Pfaff, L. (2008). Online advertising as a public health and recruitment tool: comparison of different media campaigns to increase demand for smoking cessation interventions. Journal of Medical Internet Research, 10, e50.

Griffiths, F., Lindenmeyer, A., Powell, J., Lowe, P. \& Thorogood, M. (2006). Why are health care interventions delivered over the Internet? A systematic review of the published literature. Journal of Medical Internet Research, 8, e10. 
Guimarães, N., Chambel, T. \& Bidarra, J. (2000). From cognitive maps to hypervideo: Supporting flexible and rich learner-centered environments [Online]. Available from: http://imej.wfu.edu /articles/2000/2/03 /index.asp

Gwaltney, C. J., Metrik, J., Kahler, C. W. \& Shiffman S. (2005). Dynamic Self-Efficacy and Outcome Expectancies: prediction of smoking lapse and relapse. Journal of Abnormal Social Psychology, 114, 661-675.

Gwaltney, C. J., Metrik, J., Kahler, C. W. \& Shiffman, S. (2009). Self-efficacy and smoking cessation: a meta-analysis. Psychology of Addictive Behaviours, 23, 56-66.

$\mathrm{H}$

Hakkaart-van Roijen L., Tan, S. S. \& Bouwmans, C. (2010). Handleiding voor kostenonderzoek: Methoden en standaard kostprijzen voor economusche evaluaties in de gezondheidszorg [Online]. Diemen, College voor zorgverzekeringen. Available from: http://www.cvz.nl/binaries/content/documents/zinl-www/ documenten/publicaties/overige-publicaties/1007-handleiding-voor-kostenonderzoek/Handleiding+ voor+kostenonderzoek.pdf.

Hammond, D. (2011). Health warning messages on tobacco products: A review. Tobacco Control, 20, $327-337$.

Harper, S. \& Lynch, J. (2007). Trends in socioeconomic inequalities in adult health behaviours among U.S. states, 1990-2004. Public Health Report, 122, 377-388.

Hassan E. (2006). Recall bias can be a threat to retrospective and prospective research designs. The Internet Journal of Epidemiology, 3.

Haug, S., Meyer, C., Ulbricht, S., Gross, B., Rumpf, H. J. \& John, U. (2010). Need for cognition as a predictor and a moderator of outcome in a tailored letters intervention for smoking cessation. Health Psychology, 29, 367-373.

Hawkins, R. P., Kreuter, M., Resnicow, K., Fishbein, M. \& Dijkstra, A. (2008). Understanding tailoring in communicating about health. Health Education Research, 23, 454-466.

Hays, J. T., Croghan, I. T., Baker, C. L., Cappelleri, J. C. \& Bushmakin, A. G. (2012). Changes in health-related quality of life with smoking cessation treatment. European Journal of Public Health, 22, 224-229.

Heatherton, T. F., Kozlowski, L. T., Frecker, R. C. \& Fagerström, K. O. (1991). The Fagerström Test for Nicotine Dependence: A revision of the Fagerström Tolerance Questionnaire. British Journal of Addiction, 86, 1119-1127.

Hendriks, M. R., Al, M. J., Bleijlevens, M. H., van Haastregt, J. C., Crebolder, H. F., van Eijk, J. T., \& Evers, S. M. (2013). Continuous versus intermittent data collection of health care utilization. Medical decision making: An international Journal of the Society for Medical Decision Making, 1-11. 
Heritier, S. R., Gebski, V. J. \& Keech, A. C. (2003). Inclusion of patients in clinical trial analysis: the intention-totreat principle. The Medical Journal of Australia, 179, 438-440.

Heron, K. E. \& Smyth, J. M. (2010). Ecological momentary interventions: incorporating mobile technology into psychosocial and health behaviour treatments. British Journal of Health Psychology, 15, 1-39.

Herzog, T. A. (2008). Analyzing the transtheoretical model using the framework of Weinstein, Rothman, and Sutton (1998): the example of smoking cessation. Health Psychology, 27, 548-556.

Hinyard, L. J. \& Kreuter, M. W. (2007). Using narrative communication as a tool for health behavior change: a conceptual, theoretical, and empirical overview. Health Education and Behavior, 34, 777-792.

Hiscock, R., Bauld, L., Amos, A., Fidler, J. A. \& Munafo, M. (2012). Socioeconomic status and smoking: a review. Annals of the New York Academy of Sciences, 1248, 107-123.

Hiscock, R., Judge, K. \& Bauld, L. (2011). Social inequalities in quitting smoking: what factors mediate the relationship between socioeconomic position and smoking cessation? Journal of Public Health, 33, 3947.

Hiscock, R., Murray, S., Brose, L. S., McEwen, A., Bee, J. L., Dobbie, F. \& Bauld, L. (2013). Behavioural therapy for smoking cessation: the effectiveness of different intervention types for disadvantaged and affluent smokers. Addictive Behavior, 38, 2787-2796.

Hodgson, T. A. (1998). The health care costs of smoking. The New England Journal of Medicine, 338, 470.

Hollis, S. \& Campbell, F. (1999). What is meant by intention to treat analysis? Survey of published randomised controlled trials. British Medical Journal, 319, 670-674.

Honjo, K., Tsutsumi, A., Kawachi, I. \& Kawakami, N. (2006). What accounts for the relationship between social class and smoking cessation? results of a path analysis. Social Science and Medicine, 62, 317-328.

Houts, P. S., Doak, C. C., Doak, L. G. \& Loscalzo, M. J. (2006). The role of pictures in improving health communication: a review of research on attention, comprehension, recall, and adherence. Patient Education Counseling, 61, 173-190.

Hoving, C., Mudde, A. N. \& de Vries, H. (2006a). Smoking and the O pattern: predictors of transitions through the stages of change. Health Education Research, 21, 305-314.

Hoving C, Mudde A. N. \& de Vries H. (2006b). Predictors of smoking relapse in a sample of Dutch adult smokers: the roles of gender and action plans. Addictive Behaviours, 31, 1177-1189.

Hoving, C., Mudde, A. N. \& de Vries, H. (2007). Effect of recruitment method and setting on the composition of samples consisting of adult smokers. Patient Education and Counseling, 65, 79-86. 
Hoving, C., Mudde, A. N., Dijk, F. \& de Vries H. (2010). Effectiveness of a computer-generated tailored smoking cessation expert system in Dutch community pharmacy and general practice setting. Health Education, 110, 17-29.

Hughes, J. R., Keely, J. \& Naud, S. (2004). Shape of the relapse curve and long-term abstinence among untreated smokers. Addiction, 99, 29-38.

Hughes, J. R., Keely, J. P., Niaura, R. S., Ossip-Klein, D. J., Richmond, R. L. \& Swan, G. E. (2003). Measures of abstinence in clinical trials: issues and recommendations. Nicotine Tobacco Research, 5, 13-25.

I

Irvine, A. B., Ary, D. V., Grove, D. A. \& Gilfillan-Morton, L. (2004). The effectiveness of an interactive multimedia program to influence eating habits. Health Education Research, 19, 290-305.

J

Janz, N. K., Champion, V. L. \& Strecher, V. J. (2002). The Health Belief Model. San Francisco, Jossey-Bass.

Jha, P., Peto, R., Zatonski, W., Boreham, J., Jarvis, M. J. \& Lopez, A. D. (2006). Social inequalities in male mortality, and in male mortality from smoking: indirect estimation from national death rates in England and Wales, Poland, and North America. Lancet, 368, 367-370.

K

Kessels, L., Ruiter, R., Brug, J. \& Jansma, B. (2011). The effects of tailored and threatening nutrition information on message attention. Evidence from an event-related potential study. Appetite, 56, 32-38.

Kotz, D. \& West, R. (2009). Explaining the social gradient in smoking cessation: It's not in the trying, but in the succeeding. Tobacco Control, 18, 43-46.

Krebs, P., Prochaska, J. O. \& Rossi, J. S. (2010). A meta-analysis of computer-tailored interventions for health behavior change. Preventive Medicine, 51, 214-221.

Kreuter, M. W., Farwell, D., Olevitch, L. \& Brennan, L. (1999a). Tailored Health messages: Customizing communication with computer technology. Mahwah, New Jersey, Lewrence Erlbaum.

Kreuter, M. W., Bull, F. C., Clark, E. M. \& Oswald, D. L. (1999b). Understanding how people process health information: a comparison of tailored and untailored weight loss materials. Health Psychology, 18, 487494.

Kreuter, M. W. \& Wray, R. J. (2003). Tailored and targeted health communication: Strategies for enhancing information relevance. American Journal of Health Behavior, 27, 227-232.

Krouse, H. J. (2001). Video modelling to educate patients. Journal of Advanced Nursing, 33, 748-757. 
Kunst A., Giskes, K. \& Mackenbach, J. P. (2004). Socio-economic inequalities in smoking in the European Union. Applying an equity lens to tobacco control policies. Rotterdam: Erasmus University [Online]. Available from: http://www.ensp.org/sites/default/files/ensp_socioeconomic_inequalities_in_smoking_in_eu. pdf.

L

Laaksonen, M., Rahkonen, O., Karvonen, S. \& Lahelma, E. (2005). Socioeconomic status and smoking: analysing inequalities with multiple indicators. European Journal of Public Health, 15, 262-269.

Lacey, L. P., Manfredi, C., Balch, G., Warnecke, R. B., Allen, K. \& Edwards, C. (1993). Social support in smoking cessation among black women in Chicago public housing. Public Health Reports, 108, 387-394.

Ladapo, J. A., Jaffer, F. A., Weinstein, M. C. \& Froelicher, E. S. (2011). Projected cost-effectiveness of smoking cessation interventions in patients hospitalized with myocardial infarction. Archives of Internal Medicine, $171,39-45$

Lamers, L. M., Stalmeier, P. F., McDonnell, J., Krabbe, P. F. \& van Busschbach, J. J. (2005). Measuring the quality of life in economic evaluations: the Dutch EQ-5D tariff. Nederlands Tijdschrift Geneeskunde, 149, 15741578.

Lancaster. T. \& Stead, L. (2005). Self-help interventions for smoking cessation. Cochrane Database of Systematic Reviews, CD001118.

Lee, C. W. \& Kahende, J. (2007). Factors associated with successful smoking cessation in the United States, 2000. American Journal of Public Health, 97, 1503-1509.

Lee, J. M. (2008). Effect of a large increase in cigarette tax on cigarette consumption: an empirical analysis of cross-sectional survey data. Public Health, 122, 1061-1067.

Lemmens, V., Oenema, A., Knut, I. K. \& Brug, J. (2008). Effectiveness of smoking cessation interventions among adults: a systematic review of reviews. European Journal of Cancer Prevention, 17, 535-544.

Lenert, L., Munoz, R. F., Stoddard, J., Delucchi, K., Bansod, A., Skoczen, S. \& Pérez-Stable, E. J. (2003). Design and pilot evaluation of an Internet smoking cessation program. Journal of the American Medical Informatics Association, 10, 16-20.

Leslie, E., Marshall, A. L., Owen, N. \& Bauman, A. (2005). Engagement and retention of participants in a physical activity website. Preventive Medicine, 40, 54-59.

Leung L. (2008). Internet embeddedness: links with online health information seeking, expectancy value/quality of health information websites, and Internet usage patterns. Cyberpsychology and Behavior, 11, 565569. 
Levy, D., Chaloupka, F. \& Gitchell, J. (2004). The effects of tobacco control policies on smoking rates: a tobacco control scorecard. Journal of Public Health Management Practice, 10, 338-353.

Levy, D. T., Hyland, A., Higbee, C., Remer, L. \& Compton, C. (2007). The role of public policies in reducing smoking prevalence in California: results from the California tobacco policy simulation model. Health Policy, 82, 167-185.

Lim, K. H., Sumarni, M. G., Amal, N. M., Hanjeet, K., Wan Rozita, W. M. \& Norhamimah, A. (2009). Tobacco use, knowledge and attitude among Malaysians age 18 and above. Tropical Biomedicine, 26, 92-99.

Lindstrom, M., Hanson, B. S., Ostergren, P. O. \& Berglund, G. (2000). Socioeconomic differences in smoking cessation: the role of social participation. Scandinavian Journal of Public Health, 28, 200-208.

Liu, Z. (2005). Reading behavior in the digital environment: Changes in reading behavior over the past ten years. Journal of Documentation, 61, 700-712.

Lopez, A. D., Collishaw, N. E. \& Piha, T. (1994). A descriptive model of the cigarette epidemic in developed countries. Tobacco Control, 3, 242-247.

Lorence, D. \& Park, H. (2008). Group disparities and health information: a study of online access for the underserved. Health Informatics Journal, 14, 29-38.

Lorgelly, P. K., Lawson, K. D., Fenwick, E. A. \& Briggs, A. H. (2010). Outcome measurement in economic evaluations of public health interventions: a role for the capability approach? International Journal of Environmental Research, 7, 2274-2289.

Lustria, M. L. A., Cortese, J., Noar, S. M. \& Glueckauf, R. L. (2009). Computer-tailored health interventions delivered over the Web: review and analysis of key components. Patient Education and Counseling, 74, 156-173.

M

Ma, Y., Hua, X., Lu, L. \& Zhang, H. (2005). A generic framework of user attention model and its application in video summarization. IEEE Transactions on Multimedia, 7, 907-919.

Mackenbach, J. P., Bos, V., Andersen, O., Cardano, M., Costa, G., Harding, S., Reid, A., Hemström, Ö., Valkonen, T. \& Kunst, A. E. (2003). Widening socioeconomic inequalities in mortality in six Western European countries. International Journal of Epidemiology, 32, 830-837.

Mackenbach, J. P., Stirbu, I., Roskam, A. J., Schaap, M. M., Menvielle, G., Leinsalu, M. \& Kunst, A. E. (2008). Socioeconomic inequalities in health in 22 European countries. New England Journal of Medicine, 358, 2468-2481.

Manfredi, C., Cho, Y. I., Crittenden, K. S. \& Dolecek, T. A. (2007). A path model of smoking cessation in women smokers of low socio-economic status. Health Education Research, 22, 747-756. 
Margetts, B., Vorster, H. \& Venter, C. (2003). Evidence-based nutrition: the impact of information and selection bias on the interpretation of individual studies. South African Journal of Clinical Nutrition, 16, 78-87.

Marmot, M. (2005). Social determinants of health inequalities. Lancet, 365, 1099-1104.

Marrone, G. F., Shakleya, D. M., Scheidweiler, K. B., Singleton, E. G., Huestis, M. A. \& Heishman, S. J. (2011). Relative performance of common biochemical indicators in detecting cigarette smoking. Addiction, 106, 1325-1334.

Marshall, S. W. (2007). Power for tests of interaction: Effect of raising the Type I error rate. Epidemiologic Perspective and Innovations, 4, 4.

Martinez, M. (2003). High attrition rates in e-learning: challenges, predictors, and solutions. E-Learning Developers' Journal, 14, 1-9.

Mayer, R. \& Massa, L. (2003). Three facets of visual and verbal learners: cognitive ability, cognitive style and learning preference. Journal of Education Psychology, 95, 833-846.

McClure, J. B., Greene, S. M., Wiese, C., Johnson, K. E., Alexander, G. \& Strecher, V. (2006). Interest in an online smoking cessation program and effective recruitment strategies: results from Project Quit. Journal of Medical Internet Research, 8, e14.

McDonald, P. W. (1999). Population-based recruitment for quit-smoking programs: an analytic review of communication variables. Preventive Medicine, 28, 545-557.

McGuire, W. J. (1985). Attitudes and attitude change. In M. Lindsay and E. Aronson. The Handbook of Social Psychology, New York, Random House.

Michie, S., Jochelson, K., Markham, W.A. \& Bridle, C. (2009). Low-income groups and behaviour change interventions: a review of intervention content, effectiveness and theoretical framework. Journal of Epidemiology and Community Health, 63, 610-622.

Moore, J. C., Stinson, L. L. \& Welniak, J. (2000). Income Measurement Error in Surveys: a review. Journal of Official Statistics, 16, 331-361.

Moser, D. K., Dracup, K. \& Doering, L. V. (2000). Factors differentiating dropouts from completers in a longitudinal, multicenter clinical trial. Nursing Research, 49, 109-116.

Mudde, A. N., de Vries, H. \& Strecher, V. J. (1996). Cost-effectiveness of smoking cessation modalities: comparing apples with oranges? Preventive Medicine, 25, 708-716.

Mudde A. N., Willemsen, M. C., Kremers, S. \& de Vries H. (2006). Meetinstrumenten voor onderzoek naar roken en stoppen met roken [Measurements from studies about smoking and smoking cessation]. The Hague, Dutch Expert Center for Tobacco Control (STIVORO). 
Murphy, P. W., Andrew, L., Chesson, A. L., Walker, L. \& Arnold, C. (2000). Comparing the effectiveness of video and written material for improving knowledge among sleep disorders clinic patients with limited literacy skills. South Medical Journal, 93, 297-304.

Murray, R. L., Bauld, L., Hackshaw, L. E. \& McNeill, A. (2009). Improving access to smoking cessation services for disadvantaged groups: a systematic review. Journal of Public Health, 31, 258-277.

Myung, S. K., McDonnell, D. D., Kazinets, G., Seo, H. G., \& Moskowitz, J. M. (2009). Effects of Web- and computerbased smoking cessation programs: meta-analysis of randomized controlled trials. Archives of Internal Medicine, 169, 929-937.

N

Nagelhout, G. E., de Korte-de Boer, D., van der Meer, R., Zeegers, T., van Gelder, B. \& Willemsen, M. (2011a). Theampublication: Sociaaleconomische verschillen in roken in Nederland 1988-2010 [Online]. The Hague, Dutch Expert Center for Tobacco Control (STIVORO). Available from: http://stivoro.nl/wpcontent/uploads/themapublicaties/sociaaleconomischeverschillen/Themapublicatie\%20SES.pdf.

Nagelhout, G. E., Willemsen, M. C. \& de Vries, H. (2011b). The population impact of smoke-free workplace and hospitality industry legislation on smoking behaviour. Findings from a national population survey. Addiction, 106, 816-823.

Nagelhout, G. E., de Korte-de Boer, D., Kunst, A. E., van der Meer, R. M., de Vries, H., van Gelder, B. M. \& Willemsen, M. C. (2012). Trends in socioeconomic inequalities in smoking prevalence, consumption, initiation, and cessation between 2001 and 2008 in the Netherlands. Findings from a national population survey. BMC Public Health, 12, 303.

Nagelhout, G. E., van den Putte, B., Allwright, S., Mons, U., McNeill, A., Guignard, R., Beck, F., Siahpush, M., Joossens, L., Fong, G. T., de Vries, H. \& Willemsen, M. C. (2013). Socioeconomic and country variations in cross-border cigarette purchasing as tobacco tax avoidance strategy. Findings from the ITC Europe Surveys. Tobacco Control, 23, 30-38.

Nevid, J. S., Javier, R. A. \& Moulton, J. L. (1996). Factors predicting participant attrition in a community-based, culturally specific smoking-cessation program for Hispanic smokers. Health Psychology, 15, 226-229.

Niederdeppe, J., Fiore, M. C., Baker, T. B. \& Smith, S. S. (2008a). Smoking-cessation media campaigns and their effectiveness among socioeconomically advantaged and disadvantaged populations. American Journal of Public Health, 98, 916-924.

Niederdeppe, J., Kuang, X., Crock, B. \& Skelton, A. (2008b). Media campaigns to promote smoking cessation among socioeconomically disadvantaged populations: what do we know, what do we need to learn, and what should we do now? Social Science and Medicine, 67, 1343-1355.

Nielsen, E. \& Sheppard, M. A. (1988). Television as a patient education tool: a review of its effectiveness. Patient Education and Counseling, 11, 3-16. 
Noar, S. M., Benac, C. N. \& Harris, M. S. (2007). Does tailoring matter? Meta-analytic review of tailored print health behavior change interventions. Psychological Bulletin, 133, 673-693.

0

Oenema, A., Brug, J., Dijkstra, A., de Weerdt, I. \& de Vries, H. (2008). Efficacy and use of an internet-delivered computer-tailored lifestyle intervention, targeting saturated fat intake, physical activity and smoking cessation: a randomized controlled trial. Annual Behaviour Medicine, 35, 125-135.

Oenema, A., Brug, J. \& Lechner, L. (2001). Web-based tailored nutrition education: results of a randomized controlled trial. Health Education Research, 16, 647-660.

Oka, R. K., Katapodi, M. C., Lim, J. W., Bacchetti, P. \& Froelicher, E. S. (2006). Quantifying smoking cessation outcomes: From The women's initiative for nonsmoking study $(\mathrm{X})$ : methodological implications. Nursing Research, 55, 292-297.

OSE. (2011). OverNite Software Europe BV. Available from: http://www.ose.nl/home.

P

Park, A. L., McDaid, D., Weiser, P., Von Gottberg, C., Becker, T. \& Kilian, R. (2013). Examining the cost effectiveness of interventions to promote the physical health of people with mental health problems: a systematic review. BMC Public Health, 13, 787.

Parrott, S., Godfrey, C., Raw, M., West, R. \& McNeill, A. (1998). Guidance for commissioners on the cost effectiveness of smoking cessation interventions. Health Educational Authority, 53, 1-38.

Parrott, S., \& Godfrey, C. (2004). Economics of smoking cessation. British Medical Journal, 947-949.

Patrick, D. L., Cheadle, A., Thompson, D. C., Diehr, P., Koepsell, T. \& Kinne, S. (1994). The validity of self-reported smoking: A review and meta-analysis. American Journal of Public Health, 84, 1086-1093.

Patterson, J., Eberly, L., Ding, Y. \& Hargreaves, M. (2004). Associations of smoking prevalence with individual and area level social cohesion. Journal of Epidemiology and Community Health, 58, 692-697.

Peels, D. A., Van Stralen, M. M., Bolman, C., Golsteijn, R. H. J., de Vries, H., Mudde, A. N. \& Lechner, L. (2012). Development of web-based computer-tailored advice to promote physical activity among people older than 50 years. Journal of Medical Internet Research, 14, e39.

Peters, G. J. Y., Ruiter, R. \& Kok, G. (2012) Threatening communication: A critical re-analysis and a revised metaanalytic test of fear appeal theory. Health Psychology Review, 7, 1-24. 
Peters, L. W. H., Pos, S. \& Bouwens J. (2005). Bevorderen van stoppen met roken bij achterstandsgroepen en allochtonen op lokaal niveau [Online]. Woerden, Nationaal Instituut voor Gezondheidsbevordering en Ziektepreventie (NIGZ). Available from: http://www.alcoholinfo.nl/upload/winkel/review\%20KW0 41560.

Petty, R. E., Cacioppo, J. \& Goldman, R. (1981). Personal involvement as a determinant of argument-based persuasion. Journal of Personality and Social Psychology, 41, 847-855.

Petty, R. E. \& Cacioppo, J. T. (1986). The elaboration likelihood model of persuasion. London, Academic Press.

Pisinger, C., Aadahl, M., Toft, U., \& Jorgensen, T. (2011). Motives to quit smoking and reasons to relapse differ by socioeconomic status. Preventive Medicine, 52, 48-52.

Prochaska, J. O. \& DiClemente, C. C. (1983). Stages and processes of self-change of smoking: toward an integrative model of change. Journal of Consulting and Clinical Psychology, 51, 390-395.

Prochaska, J. O., Redding, C. A. \& Evers, K. E. (1997). The transtheoretical model and stages of change. San Fransciso, Jossey-Bass.

Purcell, K. (2010). The state of online videos. Washington, PEW Research Center.

Q

Quist-Paulsen, P., Lydersen, S., Bakke, P. S. \& Gallefoss, F. (2006). Cost effectiveness of a smoking cessation program in patients admitted for coronary heart disease. European Journal of Preventive Cardiology, 13, 274-280.

R

Rasmussen, M., Due, P., Damsgaard, M. T. \& Holstein, B. E. (2009). Social inequality in adolescent daily smoking: Has it changed over time? Scandinavian Journal of Public Health, 37, 287-294.

Rasmussen, S. R., Prescott, E., Sorensen, T. I. \& Sogaard, J. (2004). The total lifetime costs of smoking. European Journal of Public Health, 14, 95-100.

Rebagliato, M. (2002). Validation of self reported smoking. Journal of Epidemiology and Community, Health, 56, 163-164.

Regidor, E. (2006). Social determinants of health: a veil that hides socioeconomic position and its relation with health. Journal of Epidemiology and Community Health, 60, 896-901.

Richardson, A., Graham, A. L., Cobb, N., Xiao, H., Mushro, A., Abrams, D. \& Vallone, D. (2013). Engagement promotes abstinence in a web-based cessation intervention: cohort study.

Journal of Medical Internet Research, 15, e14. 
Rimer, B. K. \& Kreuter, M. W. (2006). Advancing tailored health communication: A persuasion and message effects perspective. Journal of Communication, 15, 184-201.

Rise, J., Kovac, V., Kraft, P. \& Moan, I. S. (2008). Predicting the intention to quit smoking and quitting behaviour: extending the theory of planned behaviour. British Journal of Health Psychology, 13, 291-310.

Rogers E. M. (2003). Diffusion of innovations. New York, Free Press.

Ronda, G., van Assema, P., Candel, M., Ruland, E., Steenbakkers, M., van Ree, J. \& Brug, J. (2004). The Dutch Heart Health Community Intervention 'Hartslag Limburg': Effects on smoking behaviour. European Journal of Public Health, 14, 191-193.

Rooke, S., Thorsteinsson, E., Karpin, A., Copeland, J. \& Allsop, D. (2010). Computer-delivered interventions for alcohol and tobacco use: A meta-analysis. Addiction, 105, 1381-1390.

Ruiter, R. A., Kessels, L. T., Jansma, B. M. \& Brug, J. (2006). Increased attention for computer-tailored health communications: An event-related potential study. Health Psychology, 25, 300-306.

RVZ. (2006). Zinnige en duurzame zorg [Online]. Available from: http://rvz.net/uploads/docs/Advies-Zinnige_ en_duurzame_zorg.pdf.

S

Sales, M. P., Oliveira, M. I., Mattos, I. M., Viana, C. M. \& Pereira, E. D. (2009). The impact of smoking cessation on patient quality of life. The Journal Brasileiro de Pneumologia, 35, 436-441.

Salize, H. J., Merkel, S., Reinhard, I., Twardella, D., Mann, K. \& Brenner, H. (2009). Cost-effective primary carebased strategies to improve smoking cessation: more value for money. Archives of Internal Medicine, $169,230-235$.

Salomon, G. (1984). Television is easy and print is tough: The differences investment of mental effort in learning as a function of perceptions and attributions. Journal of Education Psychology, 76, 647-658.

Salomon, G. \& Gardner, H. (1986). The computer as educator: Lessons from television research. Educational Researcher, 15, 13-17.

Schaap, M. W. \& Kunst, A. E. (2009) Monitoring of socio-economic inequalities in smoking: learning from the experiences of recent scientific studies. Public Health, 123, 103-109.

Schaap, M. M., Kunst, A. E., Leinsalu, M., Regidor, E., Ekholm, O., Dzurova, D.,Helmert, U., Klumbiene, J., Santana, P. \& Mackenbach, J. P. (2008). Effect of nationwide tobacco control policies on smoking cessation in high and low educated groups in 18 European countries. Tobacco Control, 17, 248-255.

Schafer, J. L. (1999). Multiple imputation: a primer. Statistical Methods in Medical Research, 8, 3-15. 
Schneider, F., van Osch, L., Schulz, D. N., Kremers, S. P. J. \& de Vries, H. (2012). The influence of user characteristics and a periodic e-mail prompt on exposure to an Internet-delivered computer tailored lifestyle program. Journal of Medical Internet Research, 14, e40.

Schulz, D. N., Candel, M. J., Kremers, S. P., Reinwand, D. A., Jander, A. \& de Vries, H. (2013). Effects of a web-based tailored intervention to reduce alcohol consumption in adults: Randomized controlled trial. Journal of Medical Internet Research, 15, e206.

Schulz, D. N., Kremers, S. P., Vandelanotte, C., van Adrichem, M. J., Schneider, F., Candel, M. J. \& de Vries, H. (2014). Effects of a web-based tailored multiple-lifestyle intervention for adults: a two-year randomized controlled trial comparing sequential and simultaneous delivery modes. Journal of Medical Internet Research, 16, e26.

Sculpher, M. (2008). NICE's 2008 Methods Guide: Sensible consolidation or opportunities missed? Pharmacological Economics, 26, 721-724.

Secker-Walker R., Gnich, W., Platt, S. \& Lancaster, T. (2008). Community interventions for reducing smoking among adults. Cochrane Database of Systematic Reviews, CD001745.

Seidman, D. F., Westmaas, J. L., Goldband, S., Rabius, V., Katkin, E. S., Pike, K. J., Wiatrek, D., \& Sloan, R. P. (2010). Randomized controlled trial of an interactive internet smoking cessation program with long-term followup. Annals of Behavioral Medicine, 39, 48-60.

Shahab, L. \& McEwen, A. (2009). Online support for smoking cessation: a systematic review of the literature. Addiction, 104, 1792-1804.

Sheeran, P., Webb, T. L. \& Gollwitzer, P. M. (2005). The interplay between goal intentions and implementation intentions. Personality and Social Psychology Bulletin, 31, 87-98.

Shelley, D., Cantrell, M. J., Moon-Howard, J., Ramjohn, D. Q. \& VanDevanter, N. (2007). The \$5 man: the underground economic response to a large cigarette tax increase in New York City. American Journal of Public Health, 97, 1483-1488.

Shiffman, S., Patten, C., Gwaltney, C., Paty, J., Gnys, M., Kassel, J., Hickcox, M., Waters, A. \& Balabanis, M. (2006). Natural history of nicotine withdrawal. Addiction, 101, 1822-1832.

Shoham, V., Rohrbaugh, M. J., Trost, S. E., \& Muramoto, M. (2006). A family consultation intervention for healthcompromised smokers. Journal of Substance Abuse Treatment, 31, 395-402.

Siahpush, M., McNeill, A., Borland, R. \& Fong, G. T. (2006). Socioeconomic variations in nicotine dependence, selfeffiacy and intention to quit across four countries: findings from the International Tobacco Control (ITC) four country survey. Tobacco Control, 15, 71-75. 
Siegel, J. E., Weinstein, M. C., Russell, L. B. \& Gold, M. R. (1996). Recommendations for reporting costeffectiveness analyses. Panel on Cost-Effectiveness in Health and Medicine. JAMA: The Journal of the American Medical Association, 276, 1339-1341.

Sinharay, S., Stern, H. S. \& Russell, D. (2001). The use of multiple imputation for the analysis of missing data. Psycholical Methods, 6, 317-329.

Skinner, C., Strecher, V. \& Hospers, H. (1994). Physicians recommendations for mammography: do tailored messages make a difference? Journal of Public Health, 84, 43-49.

Smeets, T., Brug, J. \& de Vries, H. (2008). Effects of tailoring health messages on physical activity. Health Education Research, 23, 402-413.

Smit, E. S., de Vries, H. \& Hoving, C. (2012). Effectiveness of a web-based multiple tailored smoking cessation program: a randomized controlled trial among Dutch adult smokers. Journal of Medical Internet Research, 14, e82.

Smit, E. S., de Vries, H. \& Hoving, C. (2010). The PAS study: a randomized controlled trial evaluating the effectiveness of a web-based multiple tailored smoking cessation programme and tailored counselling by practice nurses. Contemporary Clinical Trials, 31, 251-258.

Smit, E. S, Hoving, C., Cox, V. C. M. \& de Vries, H. (2011). Influence of recruitment strategy on the reach and effect of a web-based multiple tailored smoking cessation intervention among Dutch adult smokers. Health Education Research, 27, 191-199.

Smit, E. S., Evers, S. M., de Vries, H. \& Hoving, C. (2013). Cost-effectiveness and cost-utility of Internet-based computer tailoring for smoking cessation. Journal of Medical Internet Research, 15, e57.

Smit, E. S., Fidler, J. A. \& West, R. (2011). The role of desire, duty and intention in predicting attempts to quit smoking. Addiction, 106, 844-851.

Smith, P., Frank J. \& Mustard, C. (2009) Trends in educational inequalities in smoking and physical activity in Canada: 1974-2005. Journal of Epidemiology and Community Health, 63, 317-323.

Sniehotta, F., Scholz, U. \& Schwarzer, R. (2005). Bridging the intention-behaviour gap: Planning, self-efficacy, and action control in the adoption and maintenance of physical exercise. Psychology and Health, 20, 143160 .

Soetens, K. C. M., Vandelanotte, C., de Vries H. \& Mummery, K. W. (2014). Using online computer tailoring to promote physical activity: a randomized trial of text, video and combined intervention delivery-modes, Journal of Health Communication, in press.

Song, F., Raftery, J., Aveyard, P., Hyde, C., Barton, P. \& Woolacott, N. (2002). Cost-effectiveness of pharmacological interventions for smoking cessation: a literature review and a decision analytic analysis. An international Journal of the Society for Medical Decision Making, 22, 26-37. 
Spittaels, H., De Bourdeaudhuij, I., Brug, J. \& Vandelanotte, C. (2007). Effectiveness of an online computer-tailored physical activity intervention in a real-life setting. Health Education Research, 22, 385-396.

Stanczyk, N. E., Crutzen, R., Bolman, C., Muris, J. \& de Vries, H. (2013). Influence of delivery strategy on messageprocessing mechanisms and future adherence to a dutch computer-tailored smoking cessation intervention. Journal Medical Internet Research, 15, e28.

Stanczyk, N. E., Bolman, C., Muris, J. W. \& de Vries, H. (2011). Study protocol of a Dutch smoking cessation ehealth program. BMC Public Health, 11, 847.

Steele, R., Mummery, W. K. \& Dwyer, T. (2007). Using the Internet to promote physical activity: a randomized trial of intervention delivery modes. Journal of Physical Activity and Health, 4, 245-260.

Steunbijstoppen.nl. (2012). Online stoppen met roken interventie. Available at: http://www.steunbijstoppen.nl (Archived by WebCite at http://www.webcitation.org/6BbakEW1j).

Stevens, W., Thorogood, M. \& Kayikki, S. (2002). Cost-effectiveness of a community anti-smoking campaign targeted at a high risk group in London. Health Promotion International, 17, 43-50.

Stinnett, A. A. \& Mullahy, J. (1998). Net health benefits: a new framework for the analysis of uncertainty in costeffectiveness analysis. Medical decision making: an International Journal of the Society for Medical Decision Making, 18, 68-80.

Stivoro. (2011a). Kerncijfers roken in Nederland: Een overzicht van recente Nederlandse basisgegevens over rookgedrag [Online]. The Hague, the Netherlands, Dutch Expert Center for Tobacco Control (STIVORO). Available from: http://stivoro.nl/wp-content/uploads/2012/docs/ factsheets/Kerncijfers\%20roken\%20in\%20Nederland\%202011.pdf.

Stivoro. (2011b). Trendpublicatie percentage rokers in de Nederlandse bevolking 1958-2011 [Online]. The Hague, the Netherlands, Dutch Expert Center for Tobacco Control (STIVORO). Available from: http://www.gezondezorg.org/files/Trendpublicatie\%20Percentage\%20Rokers\%202011.pdf.

Strecher, V. (2007). Internet methods for delivering behavioral and health-related interventions. Annual review of Clinical Psychology, 3, 53-76.

Strecher, V. J., McClure, J., Alexander, G., Chakraborty, B., Nair, V., Konkel, J., Greene, S., Couper, M., Carlier, C., Wiese, C., Little, R., Pomerleau, C. \&Pomerleau, O. (2008a). The role of engagement in a tailored webbased smoking cessation program: Randomized controlled trial. Journal of Medical Internet Research, 10 , e36.

Strecher, V. J., Mc Cure, J., Alexander, G., Chakraborty, B., Nair, V. \& Konkel, J. (2008b). Web-based smokingcessation programs: Results of a randomized trial. American Journal of Preventive Medicine, 34, 373-381.

Swartz, L. H., Noell, J. W., Schroeder, S. W. \& Ary, D. V. (2006). A randomised control study of a fully automated Internet based smoking cessation programme. Tobacco Control, 15, 7-12. 
Tariq, L., van Gelder, B. M., Van Zutphen, M. \& Feenstra, T. L. (2009). Smoking cessation strategies targeting people with low socio-economic status: a first exploration of the effectiveness of available interventions [Online]. Bilthoven, the Netherlands, RIVM National Institute for Public Health and the Environment. Available from: http://www.rivm.nl/dsresource?objectid=rivmp:11929\&type=org\& disposition =inline \&ns_nc=1.

Tate, D. F., Finkelstein, E. A., Khavjou, O. \& Gustafson, A. (2009). Cost effectiveness of internet interventions: Review and recommendations. Annals of Behavioural Medicine, 38, 40-45.

Te Poel, F., Bolman, C., Reubsaet, A. \& de Vries, H. (2009). Efficacy of a single computer-tailored e-mail for smoking cessation: results after 6 months. Health Education Research, 24, 930-940.

Townsend, J., Roderick, P. \& Cooper, J. (1994). Cigarette smoking by socioeconomic group, sex, and age: effects of price, income, and health publicity. British Medical Journal, 309, 923-927.

Tsourtos, G. \& O'Dwyer, L. (2008). Stress, stress management, smoking prevalence and quit rates in a disadvantaged area: has anything changed? Health Promotion Journal of Australia, 19, 40-44.

Tuong, W., Larsen, E. R. \& Armstrong, A. W. (2012). Videos to influence: a systematic review of effectiveness of video-based education in modifying health behaviors. Journal of Behavioral Medicine, 37, 218-233.

Tversky, B., Morrison, J. \& Betrancourt, M. (2002). Animation: Can it facilitate? International Journal of Human Computer Studies, 57, 247-262.

Tyler-Smith, K. (2006). Early attrition among first time eLearners: a review of factors that contribute to drop-out, withdrawal and non-completion rates of adult learners undertaking eLearning programmes. Journal of Online Learning and Teaching, 2, 73-85.

v

Van 't Riet, J., Crutzen, R. \& de Vries, H. (2010). Investigating predictors of visiting, using, and revisiting an online health-communication program: a longitudinal study. Journal of Medical Internet Research, 12, e37.

Van den Berg, M. (2003). The GPs work-related pressure. Medisch Contact, 58, 1054-1056.

Van Lenthe, F. J., Schrijvers, C. T., Droomers, M., Joung, I. M., Louwman, M. J. \& Mackenbach, J. P. (2004). Investigating explanations of socio-economic inequalities in health: the Dutch GLOBE study. European Journal of Public Health, 14, 63-70.

Van Osch, L., Lechner, L., Reubsaet, A., Wigger, S. \& de Vries, H. (2008). Relapse prevention in a national smoking cessation contest: Effects of coping planning. British Journal of Health Psychology, 13, 525-535. 
Vandelanotte, C., Duncan, M. J., Plotnikoff, R. C. \& Mummery, W. K. (2012). Do participants preferences for mode of delivery (text, video or both) influence the effectiveness of a web-based physical activity intervention? Journal of Medical Internet Research, 14, e37.

Vandelanotte, C. \& Mummery, W. K. (2011). Qualitative and quantitative research into the development and feasibility of a video-tailored physical activity intervention. The International Journal of Behavioral Nutrition and Physical Activity, 8, 70.

Vangeli, E. \& West, R. (2008). Sociodemographic differences in triggers to quit smoking: findings from a national survey. Tobacco Control, 17, 410-415.

Velicer, W. F. \& Prochaska, J. O. (2004). A comparison of four self-report smoking cessation outcome measures. Addiction Behavior, 29, 51-60.

Velicer, W. F., Prochaska, J. O., Rossi, J. S. \& Snow, M. G. (1992). Assessing outcome in smoking cessation studies. Psychological Bulletin, 111, 23-41.

Verheijden, M. W., Jans, M. P., Hildebrandt, V. H. \& Hopman-Rock, M. (2007). Rates and determinants of repeated participation in a web-based behaviour change program for healthy body weight and health lifestyle. Journal of Medical Internet Research, 9, e1.

Verplanken, B. \& Orbell, S. (2003). Reflections on past behavior. A self-report index of habit strength. Journal of Applied Social Psychology, 33, 1313-1330.

Walthouwer, M. J., Oenema, A., Soetens, K., Lechner, L. \& de Vries, H. (2013). Systematic development of a textdriven and a video-driven web-based computer-tailored obesity prevention intervention. BMC Public Health, 13, 978.

W

Wantland, D. J., Portillo, C. J., Holzemer, W. L., Slaughter, R. \& McGhee, E. M. (2004). The effectiveness of Webbased vs. non-Web-based interventions: a meta-analysis of behavioural change outcomes. Journal of Medical Internet Research, 10, e40.

Warner, K. E. (2008) Smoking cessation treatment in a public-health context. The Lancet, 371, 1976-1978.

Warren-Findlow, J., Prohaska, T. R. \& Freedman, D. (2003). Challenges and opportunities in recruiting and retaining underrepresented populations into health promotion research. Gerontologist, 43, 37-46.

Webb, T. L. (2009) Commentary on Shahab \& Mc Ewen (2009). Understanding and preventing attrition in online smoking cessation interventions: A self-regulatory perspective. Addiction, 104, 1805-1806.

Webb, T. L., Joseph, J., Yardley, L. \& Michie, S. (2010). Using the Internet to promote health behavior change: A systematic review and meta-analysis of the impact of theoretical basis, use of behavior change techniques, and mode of delivery on efficacy. Journal of Medical Internet Research, 12, e4. 
Weinstein, N. D., Rothman, A. J. \& Sutton, S. R. (1998) Stage theories of health behavior: conceptual and methodological issues. Health Psychology, 17, 290-299.

Welte, R., Koning, H. H. \& Leidl, R. (2000). The costs of health damage and productivity losses attributable to cigarette smoking in Germany. European Journal of Public Health, 10, 31-38.

Wensing, M., Vedsted, P., Kersnik, J., Peersman, W., Klingenberg, A., Hearnshaw, H., Hjortdahl, P., Paulus, D., Künzi, B., Mendive, J. \& Grol R. (2002). Patient satisfaction with availability of general practice: An international comparison. International Journal for Quality in Health Care, 14, 111-118.

West, R., Hajek, P., Stead, L. \& Stapleton, J. (2005). Outcome criteria in smoking cessation trials: Proposal for a common standard. Addiction, 100, 299-303.

West, R., \& Sohal, T. (2006). "Catastrophic" pathways to smoking cessation: findings from national survey. British Medical Journal, 332, 458-460.

White, I. R., Royston, P., \& Wood, A. M. (2011). Multiple imputation using chained equations: issues and guidance for practice. Statistics in Medicine, 30, 377-399.

World Health Organization. (2003). WHO Framework Convention on Tobacco Control [Online]. Geneva, Switzerland, World Health Organization. Available from: http://whqlibdoc.who.int/publications /2003/9241591013.pdf?ua=1.

World Health Organization. (2008). WHO report on the global tobacco epidemic, 2008: The MPOWER package [Online]. Geneva, Switzerland, World Health Organisation. Available from: http://whqlibdoc.who.int/ publications /2008/9789241596282_eng.pdf.

World Health Organization. (2010). WHO technical manual on tobacco tax administration [Online]. Geneva, Switzerland, World Health Organization. Available from: http://whqlibdoc.who.int/publications/ 2010/9789241563994_eng.pdf?ua=1.

World Health Organization. (2011). WHO report on the global tobacco epidemic, 2011: The MPOWER package [Online]. Geneva, Switzerland, World Health Organisation. Available from: http://whqlibdoc. who.int/publications/2011/9789240687813_eng.pdf.

Wiggers, L. C., Oort, F. J., Peters, R. J., Legemate, D. A., de Haes, H. C. \& Smets, E. M. (2006). Smoking cessation may not improve quality of life in atherosclerotic patients. Nicotine Tobacco Research, 8, 581-589.

Willemsen, M. C., Segaar, D. \& van Schayck, O. C. (2013). Population impact of reimbursement for smoking cessation: a natural experiment in The Netherlands. Addiction, 108, 602-604.

Wilson, E. A., Park, D. C., Curtis, L. M., Cameron, K. A., Clayman, M. L., Makoul, G., Vom Eigen, K. \& Wolf, M. S. (2010). Media and memory: the efficacy of video and print materials for promoting patient education about asthma. Patient Education and Counseling, 80, 393-398. 


\section{REFERENCES}

Wiltshire, S., Bancroft, A., Amos, A. \& Parry, O. (2001). "They're doing people a service"-qualitative study of smoking, smuggling, and social deprivation. British Medical Journal, 323, 203-207.

Woolacott, N. F., Jones, L., Forbes, C. A., Mather, L. C., Sowden, A. J., Song, F. J., Raftery, J. P., Aveyard, P. N., Hyde, C. J. \& Barton, P. M. (2002). The clinical effectiveness and cost-effectiveness of bupropion and nicotine replacement therapy for smoking cessation: a systematic review and economic evaluation. Health Technology Assessment, 6, 1-245.

z

Zebrack, J. R., Mitchel, J. L., Davids, S. L. \& Simpson, D. E. (2005). Web-based curriculum. A practical and effective strategy for teaching women's health. Journal of General Internal Medicine, 20, 68-74. 


\section{APPENDICES}

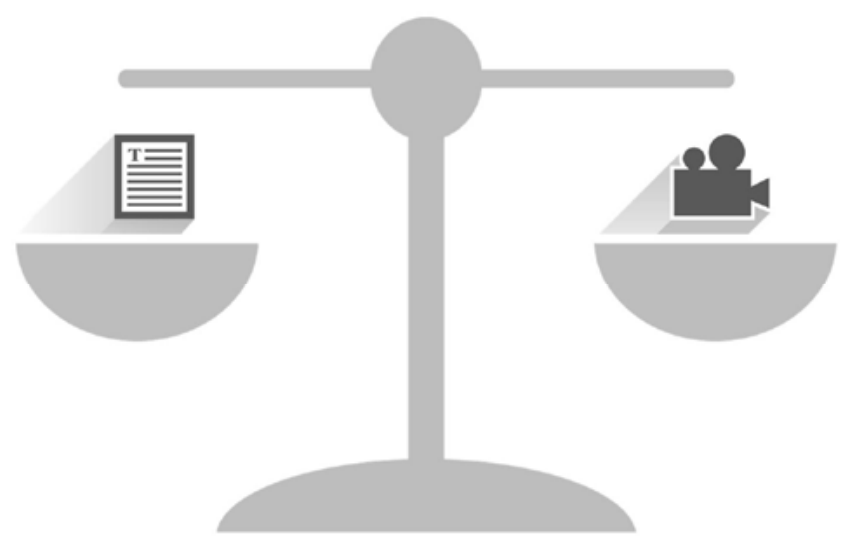





\section{Appendix 1}

\section{Results of regression analysis on sample 2}

Table 1 Six-month abstinence rates (seven-day point prevalence abstinence and prolonged abstinence) per condition for sample 2 (negative scenario)

\begin{tabular}{llllll}
\hline Negative scenario & Total $(\mathrm{N})$ & Video & Text & Control & $p$ \\
\hline Seven-day PPA ; \% (n) & 1,462 & $30.6(90)$ & $22.6(101)$ & $14.6(105)$ & .000 \\
PA b; \% (n) & 1,462 & $22.8(67)$ & $33.3(77)$ & $37.7(87)$ & .000 \\
PA ; \% (n) & 1,462 & & & & \\
Readiness to quit within 1 month & $25.1(45)$ & $21.4(60)$ & $15.2(52)$ & .02 \\
Readiness to quit within 1-3 months & $17.1(13)$ & $10.6(11)$ & $13.2(30)$ & .44 \\
Readiness to quit within 4-6 months & $23.1(9)$ & $9.5(6)$ & $3.3(5)$ & .000 \\
\hline
\end{tabular}

Note: $p$-values <.05 are marked bold; ${ }^{a}$ point prevalence abstinence (PPA); ${ }^{b}$ prolonged abstinence (PA).

Table 2 Factors associated to seven-day point prevalence abstinence in samples 2 (negative scenario) in the present study

\begin{tabular}{|c|c|c|c|}
\hline \multirow{2}{*}{$\begin{array}{l}\text { Negative scenario } \\
\text { Variable } \\
\end{array}$} & \multicolumn{3}{|c|}{ Sample $2(N=1,462)$} \\
\hline & OR & $95 \% \mathrm{Cl}$ & $p$ \\
\hline VCT vs. CC & 2.29 & $1.64-3.20$ & .000 \\
\hline TCT vs. CC & 1.57 & $1.15-2.15$ & .005 \\
\hline Gender (male) & 0.88 & $0.66-1.16$ & .35 \\
\hline Age & 1.01 & $0.99-1.02$ & .19 \\
\hline Dutch nationality & 1.24 & $0.65-2.39$ & .51 \\
\hline Middle education level ${ }^{a}$ & 1.13 & $0.81-1.57$ & .46 \\
\hline High education level ${ }^{a}$ & 1.07 & $0.75-1.52$ & .70 \\
\hline Readiness to quit within 1 month $^{\mathrm{b}}$ & 1.64 & $1.06-2.55$ & .02 \\
\hline Readiness to quit within $1-3$ months $^{b}$ & 1.40 & $0.88-2.22$ & .16 \\
\hline FTND score & 0.94 & $0.89-1.00$ & .05 \\
\hline CES-D score & 0.94 & $0.89-1.00$ & .07 \\
\hline With COPD ${ }^{\mathrm{c}}$ & 0.93 & $0.60-1.43$ & .74 \\
\hline With cancer ${ }^{\mathrm{c}}$ & 0.89 & $0.31-2.50$ & .82 \\
\hline With diabetes ${ }^{c}$ & 0.98 & $0.53-1.84$ & .96 \\
\hline With cardiovascular diseases ${ }^{c}$ & 1.17 & $0.74-1.86$ & .49 \\
\hline With asthma ${ }^{\mathrm{c}}$ & 1.21 & $0.69-2.14$ & .50 \\
\hline Recruitment strategy & 0.63 & $0.41-0.98$ & .04 \\
\hline \multicolumn{4}{|l|}{ Newspaper/Internet ${ }^{d}$} \\
\hline Preparatory planning & 1.09 & $1.03-1.15$ & .005 \\
\hline Coping planning & 0.99 & $0.91-1.08$ & .87 \\
\hline Self-efficacy & 1.15 & $0.98-1.35$ & .08 \\
\hline
\end{tabular}

Note: $p$-values < .05 are marked bold; interaction terms are not included in the final model since they were not significant and ORs are adjusted for variables significant at baseline and drop-out; ${ }^{a}$ low education is the reference category; ${ }^{b}$ willingness to quit within 4-6 months is the reference category; ${ }^{c}$ not suffering from the disease is the reference category; ${ }^{d}$ general practitioner (GP) is the reference category. 
Table 3 Factors associated to prolonged abstinence in samples 2 (negative scenario) in the present study

\begin{tabular}{|c|c|c|c|}
\hline \multirow{2}{*}{$\begin{array}{l}\text { Negative scenario } \\
\text { Variables }\end{array}$} & \multicolumn{3}{|c|}{ Sample $2(N=1,462)$} \\
\hline & OR & $95 \% \mathrm{Cl}$ & $p$ \\
\hline VCT vs. CC & 8.99 & $2.75-29.41$ & .000 \\
\hline TCT vs. CC & 3.13 & 0.91-10.77 & .07 \\
\hline Gender (male) & 0.77 & $0.56-1.05$ & .10 \\
\hline Age & 1.02 & $1.00-1.03$ & .030 \\
\hline Dutch nationality & 1.12 & $0.52-2.40$ & .77 \\
\hline Middle Education level ${ }^{a}$ & 1.08 & $0.75-1.55$ & .69 \\
\hline High education level ${ }^{a}$ & 0.91 & $0.62-1.34$ & .63 \\
\hline Readiness to quit within 1 month $^{b}$ & 4.14 & 1.59-10.79 & .004 \\
\hline Readiness to quit within $1-3$ months $^{b}$ & 4.10 & $1.59-10.79$ & .005 \\
\hline FTND score & 0.95 & 0.89-1.01 & .09 \\
\hline CES-D score & 0.91 & $0.85-0.98$ & .01 \\
\hline With COPD ${ }^{\mathrm{c}}$ & 1.20 & $0.74-1.95$ & .46 \\
\hline With cancer ${ }^{c}$ & 0.69 & $0.24-1.97$ & .49 \\
\hline With diabetes ${ }^{c}$ & 0.90 & $0.46-1.77$ & .77 \\
\hline With cardiovascular diseases ${ }^{c}$ & 1.34 & $0.80-2.24$ & .27 \\
\hline With asthma ${ }^{c}$ & 1.22 & $0.65-2.30$ & .54 \\
\hline Recruitment strategy & 0.63 & $0.39-1.01$ & .05 \\
\hline \multicolumn{4}{|l|}{ Newspaper/Internet ${ }^{d}$} \\
\hline Preparatory planning & 1.12 & $1.05-1.20$ & .001 \\
\hline Coping planning & 1.06 & $0.96-1.18$ & .24 \\
\hline Self-efficacy & 1.16 & $0.98-1.39$ & .09 \\
\hline \multicolumn{4}{|l|}{ Interaction } \\
\hline High readiness to quit $*$ VCT & 0.18 & $0.05-0.66$ & .009 \\
\hline High readiness to quit $*$ TCT & 0.47 & $0.13-1.73$ & .25 \\
\hline Middle readiness to quit $* \mathrm{VCT}$ & 0.13 & $0.03-0.53$ & .004 \\
\hline Middle readiness to quit $*$ TCT & 0.24 & $0.05-0.99$ & .05 \\
\hline \multicolumn{4}{|l|}{ Subgroup analyses } \\
\hline \multicolumn{4}{|l|}{ Readiness to quit within 1 month } \\
\hline$\overline{\text { VCT vs. TCT }}$ & 1.13 & $0.71-1.79$ & .60 \\
\hline VCT vs. CC & 1.65 & $1.04-2.63$ & .03 \\
\hline TCT vs. CC & 1.46 & $0.95-2.24$ & .08 \\
\hline \multicolumn{4}{|l|}{ Readiness to quit within 1-3 months } \\
\hline VCT vs. TCT & 1.61 & $0.66-3.91$ & .29 \\
\hline VCT vs. CC & 1.18 & $0.57-2.46$ & .65 \\
\hline TCT vs. CC & 0.74 & $0.35-1.56$ & .42 \\
\hline \multicolumn{4}{|l|}{ Readiness to quit within 4-6 months } \\
\hline$\overline{\text { VCT vs. TCT }}$ & 2.88 & $0.91-9.06$ & .07 \\
\hline VCT vs. CC & 8.99 & $2.75-29.41$ & .000 \\
\hline TCT vs. CC & 3.13 & 0.91-10.77 & .07 \\
\hline
\end{tabular}

Note: $p$-values <.05 are marked bold and ORs are adjusted for variables significant at baseline and drop-out; ${ }^{a}$ low education is the reference category; ${ }^{b}$ willingness to quit within 4-6 months is the reference category; ${ }^{c}$ suffering not from the disease is the reference category; ${ }^{d}$ general practitioner (GP) is the reference category. 


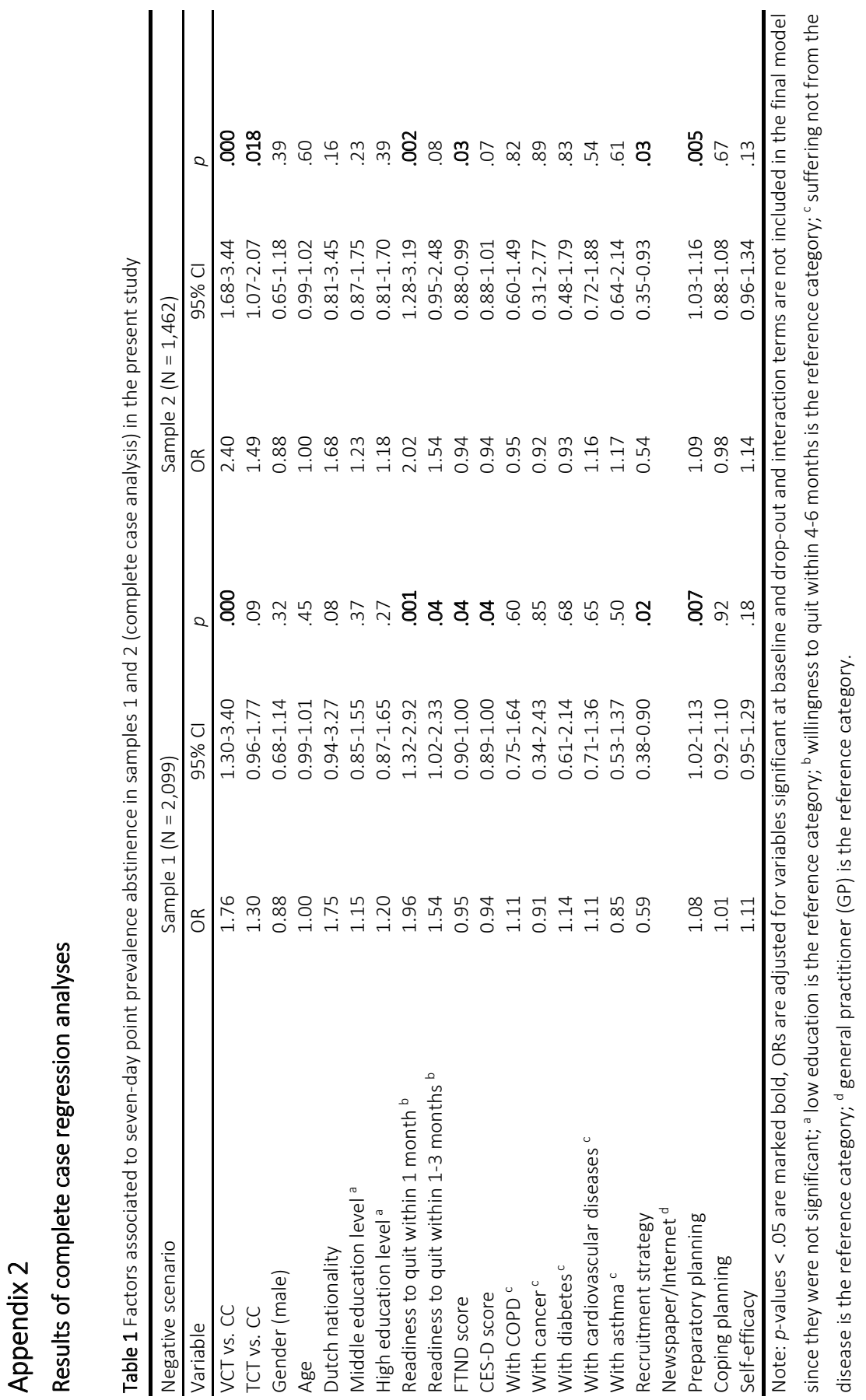




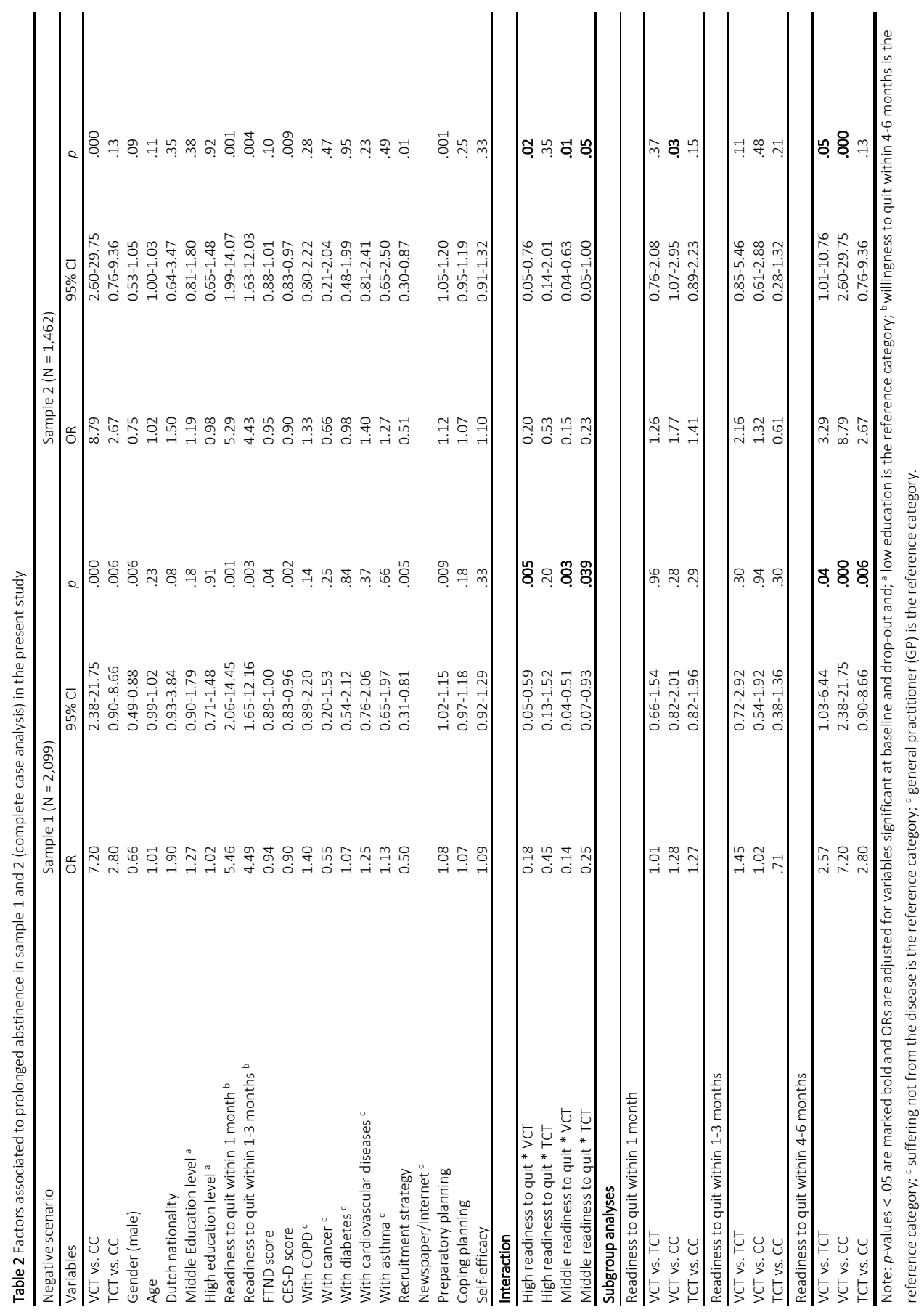


SUMMARY

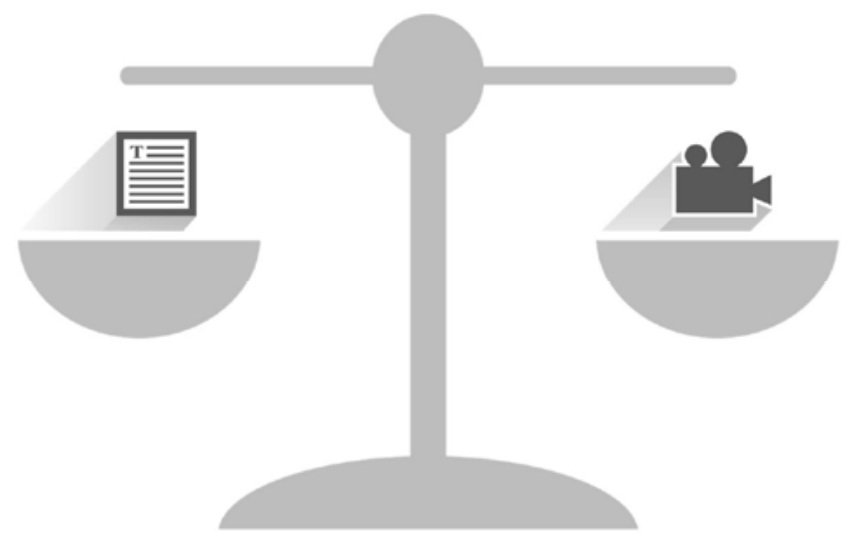



Tobacco use is still one of the leading causes of illnesses and death in the Netherlands. Although smoking increases the risk of premature death among all consumers, the problem of tobacco use has become most salient for people with a lower socioeconomic status (LSES). Smoking prevalence is highest among LSES smokers and compared to smokers with higher socioeconomic status (HSES) they often have a lower motivation to quit, show fewer attempts to quit and more often relapse to smoking. Although computer-tailored smoking cessation interventions have shown to be effective, results from previous research have shown that they often have difficulties attracting LSES smokers; reach and adherence in these interventions is especially low among LSES smokers. Hence, it is important to assess how these interventions can be improved to stimulate and attract different socioeconomic status (SES) groups, especially LSES groups. One strategy could be the use of video-delivered information. Earlier research has indicated that video information might be more effective in attracting LSES groups and to stimulate their comprehension of the smoking cessation messages. Furthermore, another problem concerns the motivation to quit smoking. Most of today's computer-tailored smoking cessation interventions have been developed for highly motivated smokers. However, particularly LSES, who are often less motivated to quit, and unmotivated smokers in general might profit from interventions that stimulate them to reflect on their smoking behaviour and their intention to quit. Accordingly, two multiple web-based computer-tailored smoking cessation interventions were developed, incorporating different delivery approaches (videovs. text-based computer-tailored messages) and two different motivational routings to target smokers with different motivational and SES levels. One of the interventions provided respondents with text-based messages whereas the other intervention provided respondents with video-based messages.

First, the current dissertation aimed to provide more insight into the reach, adherence and processing of the two computer-tailored smoking cessation interventions among smokers with different SES levels (Chapter 3 and Chapter 4). Second, the dissertation aimed to evaluate the two computer-tailored interventions with regard to effectiveness and cost-effectiveness (Chapter 5, 6 and 7).

Chapter 1 gives a general introduction to the studies presented in this dissertation. The background, the theoretical framework for the intervention as well as the different research questions of the current dissertation is presented. Moreover, an overview of each of the studies is provided at the end of this chapter.

Chapter 2 provides a detailed description of the development and design of the two computer-tailored smoking cessation interventions to increase smoking abstinence among LSES and HSES smokers.

Chapter 3 describes a study investigating the reach, retention and costs of four different recruitment strategies (i.e. General practitioners (GPs), Internet, newspaper and other mailing organisations) aimed to recruit smokers ( $N=832)$ for participation in the 
computer-tailored smoking cessation intervention. Recruitment via GPs reached a larger proportion of lower educated smokers and smokers with smoking-related diseases. Respondents recruited via GPs were more likely to make a quit attempt as well as more likely to maintain non-smoking compared with the other recruitment strategies. Yet, in absolute numbers, more smokers with a lower education level were reached by the newspaper, Internet and mailing strategies. The recruitment via mass media channels (e.g. newspapers and the Internet) might be therefore a better and lower-priced approach to reach a higher number of smoker's in future web-based computer-tailored smoking cessation interventions.

Chapter 4 describes a study investigating whether differences exist in messageprocessing mechanisms (i.e. attention, comprehension, self-reference, appreciation, processing) and future adherence (intention to visit/use the website again; recommend the website to others), according to the delivery strategy (video vs. text-based messages) and SES (SES; indexed by educational level) for the computer-tailored smoking cessation intervention among smokers $(N=139)$. Results showed that LSES smokers paid more attention to the computer-tailored messages and seemed to process the information more intensely. Additionally, they were more inclined to revisit the website compared to HSES smokers. Processing of tailored messages was not influenced by delivery strategy. The findings suggest that the computer-tailored programs have the potential to positively influence LSES groups as they might be more involved in these programs compared to HSES smokers.

Chapter 5 describes the randomised controlled trial (RCT), investigating the short-term effectiveness (six months after baseline measurement) of the video-based and text-based computer-tailored intervention compared to a control condition. Moreover, differential effects of the intervention among different SES levels and different motivational levels were assessed. Smokers willing to quit within six months were assigned to the video-based condition $(n=670)$, the text-based condition $(n=708)$ or the control condition (receiving general text advice $(n=$ 721). Analyses were conducted in two different samples. The first sample included all randomly assigned respondents that completed the baseline questionnaire and session 1 (directly after baseline). The second sample included only respondents in the experimental conditions who completed at least one further session of the intervention. All respondents of the control condition were just normally included. No significant differences were found between LSES, middle SES (MSES) and HSES smokers (negative scenario; primary analysis) in both samples on the outcome variables seven-day point prevalence abstinence and prolonged abstinence. However, the results showed that in sample 1, the video-based computer-tailored intervention was 'overall' effective in increasing seven-day point prevalence abstinence compared to the control condition. Furthermore, the video-based intervention was particularly effective in increasing prolonged abstinence among respondents with a low (within four to six months) readiness to quit smoking. Similar results for both outcomes were found in sample 2 . In addition, in sample 2 , the text-based condition was more effective in realising seven-day point 
prevalence abstinence compared to the control condition. Complete case and multiple imputation analyses showed comparable results. Lastly, the video feedback messages seem to be slightly better evaluated than the text-based messages in terms of appreciation and processing, but differences did not reach significance.

Chapter 6 describes the RCT, investigating the long-term effectiveness (12-months after baseline measurement) of the video-based and text-based computer-tailored intervention compared to a control condition, and differential effects of the intervention for smokers with different SES and motivational levels. No differences in effects were found for people with a high or low motivation to quit smoking. Additionally, no significant differences in abstinence rates were found for people with a LSES and HSES (multiple imputation; primary analyses). However, the video-based computer-tailored intervention resulted in significantly higher prolonged abstinence rates. Comparable results were found in the complete cases and negative scenario approach. For seven-day point prevalence abstinence, we were neither able to replicate our findings with the multiple imputation analyses nor complete cases or negative scenario. These findings suggest that the video-based computer-tailored intervention is effective in helping smokers to achieve prolonged abstinence. The results support the effectiveness of using web-based computer-tailored video messaging to influence smoking behaviour.

Chapter 7 presents the cost-effectiveness and cost-utility of the two computer-tailored smoking cessation interventions (video-based vs. text-based messages) compared to the control condition after 12 months. The cost-effectiveness analysis, using prolonged abstinence as the main outcome measure, showed that from a willingness to pay of $€ 1,500$, the videobased intervention was likely to be the most cost-effective treatment, whereas from a willingness to pay of $€ 50,400$, the text-based intervention was likely to be most cost-effective. Regarding cost-utilities, when quality of life was used as an outcome measure, the control condition was probably most efficient. Difficulties in refraining from smoking and several health problems due to withdrawal may have diminished improvements in quality of life. For proper assessments of cost-utility, more time will be required in order to be able to measure changes in quality of life validly. Sensitivity analyses showed comparable results.

In Chapter 8, the general discussion of this dissertation, the different results of Chapter's 2 to 7 are integrated and the main findings are related to previous research. Furthermore, several methodological considerations and implications for future research and practice are discussed. Several conclusions can be drawn. While recruitment via GPs resulted in a higher percentage of LSES smokers who quit smoking, recruitment via mass media approaches (such as the Internet and newspaper advertising) resulted in a larger absolute number of smokers who made a quit attempt. Next, although delivery strategy (video-based vs. text-based messages) seemed to play no role in the processing of the tailored information, LSES smokers showed higher attention and processing levels of the tailored messages and were 
more inclined to visit the computer-tailored intervention again compared to HSES smokers. Furthermore, the video-based computer-tailored intervention was especially effective in increasing short-term abstinence compared to the control condition in smokers with a lower motivation to quit smoking on the most valid outcome measurement, namely prolonged abstinence. Moreover, the video-based computer-tailored intervention was effective for achieving long-term smoking abstinence. No differential effects were found for LSES, MSES and HSES smokers. Additionally, the video-based computer-tailored intervention was also the most cost-effective treatment for smoking abstinence. Since the video-based intervention showed more effects and lower costs, we advise the implementation of video-based messages above text-based messages. Even though the video-based intervention was successful in increasing short- and long-term smoking abstinence rates, the intervention may benefit from further improvement to optimise its potential. 
SAMENVATTING

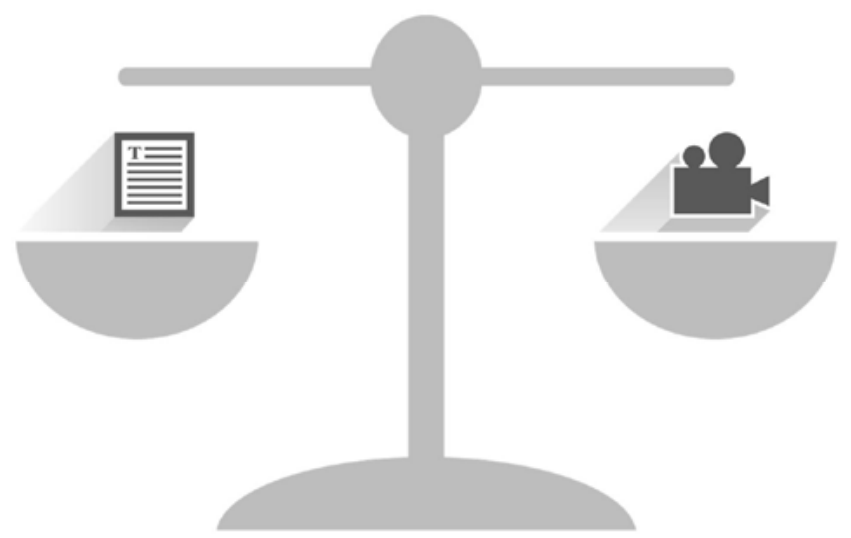



Roken van tabak is in Nederland één van de belangrijkste oorzaken van ziekte en vroegtijdige sterfte. Hoewel roken het risico op vroegtijdig overlijden onder alle rokers verhoogt, is roken vooral een probleem onder mensen met een lagere sociaal economische status (LSES). De rookprevalentie is op dit moment het hoogst bij rokers met een LSES. Zij zijn vaak minder gemotiveerd om te stoppen met roken, zijn minder succesvol in hun stoppogingen en beginnen vaak sneller met roken vergeleken met rokers met een hogere sociaal economische status (HSES). Ondanks het feit dat stoppen-met-roken advies-op-maat interventies effectief blijken te zijn, heeft onderzoek op dit gebied uitgewezen dat het bereik en het gebruik van deze interventies vaak laag is onder LSES rokers. Daarom is het belangrijk om te onderzoeken hoe deze interventies zodanig verbeterd kunnen worden dat ze aantrekkelijk zijn voor verschillende sociaal economische groepen (SES) en in het bijzonder voor groepen met een LSES. Het gebruik van video-informatie zou een strategie kunnen zijn. Eerder onderzoek heeft laten zien dat video/beeldmateriaal mogelijk een geschikt kanaal is om informatie over te brengen en dat het effectief kan zijn in het stimuleren van LSES groepen om te stoppen met roken. Een ander probleem bij stoppen-met-roken interventies betreft de motivatie om te stoppen met roken. De meeste van de hedendaagse stoppen-met-roken advies-op-maat interventies zijn ontwikkeld voor gemotiveerde rokers. Echter, LSES rokers, die vaak minder gemotiveerd zijn om te stoppen met roken, en ongemotiveerde rokers in het algemeen, zijn mogelijk meer gebaat bij interventies die hen stimuleren om na te denken over hun voornemen om te stoppen met roken. Gebaseerd op deze kennis zijn twee online stoppen-met-roken advies-opmaat interventies ontwikkeld. Deze interventies verschillen in aanbiedingsvorm: een online tekst advies-op-maat interventie, waarbij rokers persoonlijke geschreven tekstboodschappen ontvangen en een online video advies-op-maat interventie, waarbij rokers persoonlijke video boodschappen ontvangen. Afhankelijk van de motivatie om te stoppen met roken konden de respondenten twee verschillende motivationele routes volgen.

Het eerste doel van dit proefschrift was om het bereik, het gebruik en de verschillende verwerkingsmechanismen (het proces om informatie te verwerken) te vergelijken en te onderzoeken (Hoofdstuk 3 en Hoofdstuk 4). Het tweede doel was om de twee interventies te evalueren wat betreft de effectiviteit en kosteneffectiviteit (Hoofdstuk 5, 6 en 7).

Hoofdstuk 1 geeft een algemene introductie van de studies die in dit proefschrift gepresenteerd zijn. De achtergrond, het theoretische kader voor de interventie als ook de verschillende onderzoeksvragen worden beschreven. Bovendien is er een overzicht gegeven van de studies die in het proefschrift aan bod komen.

Hoofdstuk 2 geeft een beschrijving van de ontwikkeling en het ontwerp van de twee online advies-op-maat interventies om stoppen met roken te bevorderen onder zowel LSES als ook HSES rokers.

Hoofdstuk 3 beschrijft een studie die het bereik, het gebruik, het behoud en de kosten van vier verschillende wervingsstrategieën (huisartsen, internet, kranten en andere mailing 
organisaties) onderzocht voor deelname aan de stoppen-met-roken advies-op-maat interventie. Er werden 832 rokers geworven. Werving via huisartsen bereikte een groter deel van de lager opgeleide rokers en rokers met rook-gerelateerde ziektes. Respondenten die werden geworven via de huisartsenpraktijk hadden zowel een grotere kans om een stoppoging te doen als een grotere kans om stoppen-met-roken vol te houden, vergeleken met de andere wervingsstrategieën. In absolute aantallen werden er echter meer laag opgeleide rokers bereikt met de krant, internet en mailing strategieën. Voor toekomstige online stoppen-metroken advies-op-maat interventies zou daarom de werving via massamedia kanalen (bijvoorbeeld kranten en/of internet) een betere en goedkopere benadering zijn. Op deze manier zijn in verhouding meer rokers te bereiken.

Hoofdstuk 4 beschrijft een studie naar de verschillen in verwerkingsmechanismen (aandacht, begrip, zelf-reflectie, waardering en verwerking) en toekomstig gebruik (intentie om de interventie website opnieuw te bezoeken of de website aan anderen aan te bevelen) van de twee stoppen-met-roken advies-op-maat interventies (video- versus tekst-gebaseerde berichten). Hierbij is tevens gekeken naar verschillen tussen rokers van verschillende SES groepen (SES is geïndexeerd door opleidingsniveau) $(N=139)$. De resultaten lieten zien dat LSES rokers meer aandacht besteden aan de advies-op-maat berichten dan HSES rokers en dat zij de informatie intensiever verwerkten. Daarnaast hadden ze ook vaker de intentie om de website opnieuw te bezoeken vergeleken met HSES rokers. De verwerking van de advies-opmaat boodschappen werd niet beïnvloed door het type aanbiedingsvorm (tekst versus video). De bevindingen suggereren dat advies-op-maat programma's de potentie hebben om LSES groepen positief te beïnvloeden, aangezien ze meer betrokken waren bij het programma ten opzichte van HSES rokers.

Hoofdstuk 5 beschrijft een gerandomiseerd gecontroleerd experiment (Engels: RCT, voor randomized controlled trial), dat de korte-termijn effectiviteit (zes maanden na de nulmeting) van de video- en tekst-gebaseerde advies-op-maat interventie onderzocht in vergelijking tot een controlegroep. Bovendien zijn differentiële effecten van de interventie onderzocht bij mensen met verschillende SES en bij mensen met een lage, middelmatige en hoge motivatie om te stoppen met roken. Rokers die bereid waren om binnen zes maanden te stoppen met roken werden toegewezen aan de video groep $(n=670)$, de tekst groep $(n=708)$ of de controlegroep $(n=721)$. Vervolgens zijn de analyses als volgt uitgevoerd. Allereerst zijn alle respondenten (zoals ze waren toegewezen aan de drie hiervoor genoemde groepen) geanalyseerd (eerste groep). Voor de respondenten in de experimentele groepen (videoversus tekst-gebaseerde berichten) gold hierbij dat ze de nulmeting en sessie 1 (direct na de nulmeting) hadden afgerond. Vervolgens zijn alleen respondenten geanalyseerd die minimaal nog een nieuwe bijeenkomst van de interventie hadden doorlopen (in aanvulling op sessie 1). De tweede groep was dus kleiner. Er werden geen significante verschillen gevonden tussen LSES, midden SES (MSES) en HSES rokers ('negative scenario'; primaire analyses) in de twee 
groepen op zevendaagse puntprevalentie abstinentie en geprolongeerde abstinentie. De resultaten lieten echter in de eerste groep zien dat de video-gebaseerde advies-op-maat interventie effectief was in het verhogen van zevendaagse puntprevalentie abstinentie vergeleken met de controlegroep. Bovendien was de video-gebaseerde interventie in het bijzonder effectief in het verhogen van geprolongeerde abstinentie bij mensen met een lage motivatie om te stoppen met roken. Vergelijkbare resultaten voor beide uitkomstmaten werden in de tweede groep gevonden. Verder was de tekst-gebaseerde interventie ook effectief in het realiseren van zevendaagse punt prevalentie abstinentie in de tweede groep vergeleken met de controlegroep. 'Complete case analyses' en 'multiple imputation analyses' toonden vergelijkbare resultaten. Tenslotte werden de video-gebaseerde berichten iets beter geëvalueerd dan de tekst-gebaseerde berichten in termen van waardering en verwerking. Deze verschillen waren echter net niet significant.

Hoofdstuk 6 beschrijft dezelfde RCT als in hoofdstuk 5, maar dan met betrekking tot de lange-termijn effectiviteit (12 maanden na de nulmeting) van de video- en tekst-gebaseerde advies-op-maat interventie in vergelijking tot een controlegroep. Hierbij werd ook weer onderzocht of er differentiële effecten van de interventie waren voor rokers van verschillende SES groepen en voor rokers met verschillende motivationele niveaus. Er zijn geen verschillen gevonden in effecten voor mensen met een hoge of lage motivatie om te stoppen met roken. Bovendien werden er geen significante verschillen gevonden op abstinentie voor mensen met een LSES en HSES ('multiple imputation'; primaire analyses). Er werden echter wel interventie effecten gevonden op geprolongeerde abstinentie in de video-gebaseerde interventie. Vergelijkbare resultaten werden gevonden in de 'complete cases' en de 'negative scenario' aanpak. Voor zevendaagse puntprevalentie waren we niet in staat om de resultaten te repliceren met de 'multiple imputation' analyses noch met de 'complete cases' of 'negative scenario' aanpak. De bevindingen indiceren dat de video-gebaseerde interventie effectief is in het bevorderen van geprolongeerde abstinentie. Dit betekent dat de advies-op-maat video boodschappen het rookgedrag effectief kunnen beïnvloeden.

In hoofdstuk 7 werd de kosteneffectiviteit en kostenutiliteit gepresenteerd van de twee advies-op-maat interventies (video- versus tekst-gebaseerde berichten) in vergelijking met de controlegroep na 12 maanden. Bij de kosten-baten analyse, waarbij geprolongeerde abstinentie als uitkomstmaat werd gebruikt, bleek dat bij een betalingsbereidheid (in vakjargon 'willingness to pay') van $€ 1.500$, de video-gebaseerde interventie de meest kosteneffectieve behandeling is, terwijl bij een 'willingness to pay' van $€ 50.400$, de tekst-gebaseerde interventie waarschijnlijk het meest kosteneffectief is. Wat betreft kostenutiliteit, waarbij kwaliteit van leven als uitkomstmaat werd gebruikt, is de controlegroep, dus het niet aanbieden van een interventie, het meest efficiënt. Moeilijkheden tijdens het volhouden van de stoppoging en het niet direct bemerken van positieve gezondheidseffecten kunnen een mogelijke verklaring zijn waarom respondenten geen hogere kwaliteit van leven rapporteren na het deelnemen aan de 
interventie in vergelijking met de controlegroep. Voor een goede evaluatie van de kostenutiliteit is waarschijnlijk meer tijd nodig om positieve veranderingen in levenskwaliteit te kunnen meten. Sensitiviteitsanalyses toonden vergelijkbare resultaten.

Hoofdstuk 8 omvat de algemene discussie van dit proefschrift. De resultaten die in hoofdstuk 2 tot met 7 zijn gepresenteerd, worden hier geïntegreerd en gerelateerd aan de resultaten van eerder onderzoek. Daarnaast worden methodologische beperkingen en implicaties voor toekomstig onderzoek en praktijk behandeld. Op basis hiervan worden in dit hoofdstuk een aantal conclusies getrokken. Terwijl de werving via huisartsen zorgde voor een hoger percentage van LSES rokers voor deelname aan het interventieonderzoek die ook daadwerkelijk een stoppoging ondernamen, resulteerde de werving via de massa media aanpak (zoals internet en kranten) in een groter absoluut aantal rokers die een stoppoging deden. Terwijl de aanbiedingsvorm (video-vs. tekst-gebaseerde boodschappen) geen rol leek te spelen bij de verwerking van de advies-op-maat informatie, lieten de resultaten zien dat LSES rokers meer aandacht besteedden aan de advies-op-maat berichten, de informatie dieper verwerkten en een grotere intentie hadden om de advies-op-maat interventie opnieuw te bezoeken, vergeleken met HSES rokers. Bovendien was de video-gebaseerde advies-op-maat interventie vooral effectief in het verhogen van de korte-termijn abstinentie ten opzichte van de controlegroep bij rokers met een lagere motivatie om te stoppen met roken op de meest valide uitkomstmaat, namelijk geprolongeerde abstinentie. Er zijn geen differentiële effecten gevonden voor LSES, MSES en HSES rokers. Daarnaast was de video-gebaseerde advies-opmaat interventie ook de meest kosteneffectieve behandeling voor rookabstinentie. Aangezien de video-gebaseerde interventie effectief is tegenover de controlegroep en lagere kosten met zich meebrengt, wordt implementatie van video-gebaseerde boodschappen aanbevolen boven tekst-gebaseerde boodschappen. Hoewel de video-gebaseerde interventie succesvol was in het verhogen van rookabstinentie op korte en lange termijn, dient men een aantal aanbevelingen voor verbetering van de interventie in oogschouw te nemen. 
CURRICULUM VITAE

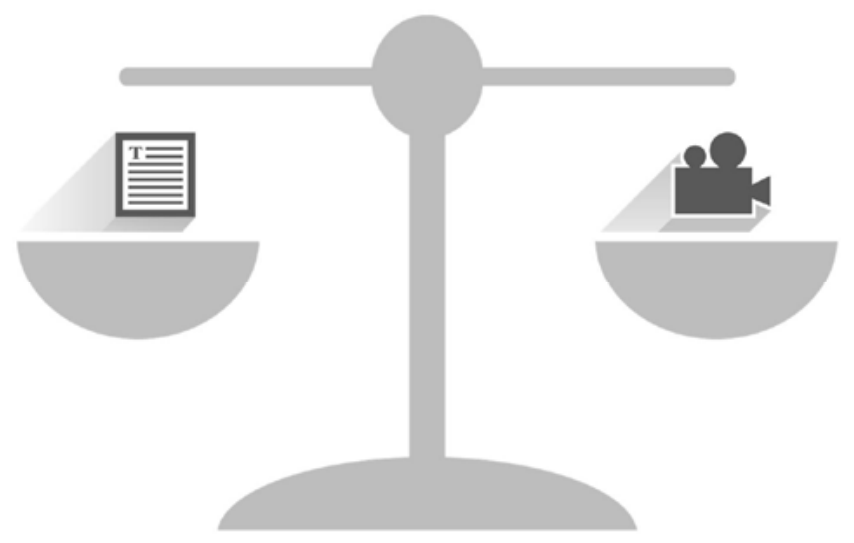





\section{Biography}

Nicola Esther Stanczyk was born on November 12, 1986 in Cologne, Germany. After receiving her Gymnasium diploma from the Erzbischhoefliche Liebfrauenschule in Bonn (Germany), she moved to Maastricht to study Psychology at Maastricht University. During her Bachelor curriculum, she followed several elective courses at the University of Sussex in the United Kingdom. She obtained her degree in 2009, after which she started a Master's program in Social and Health Psychology at Maastricht. She obtained her Master's degree in 2010.

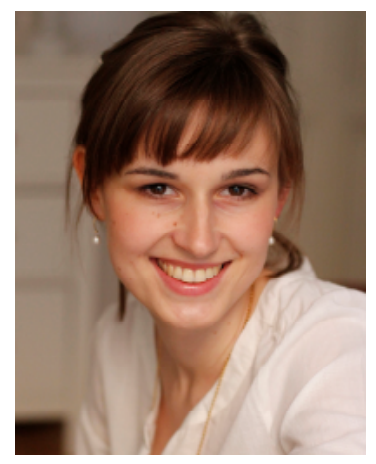
Later that year, she began working as a PhD student at the department of Health Promotion at Maastricht University on the project which is described in this dissertation. Nicola has presented her research at different national and international conferences. Since June 2014, Nicola started to work at the Elkerliek hospital in Helmond as a project manager to facilitate the implementation of an electronic medication prescription system.

\section{Publications}

d.d. 03-08-2014

\section{Publications from this thesis}

Stanczyk, N. E., Bolman, C., Muris, J. W. M., de Vries, H. (2011). Study protocol of a Dutch smoking cessation e-health program. BMC Public Health, 11, 847.

Stanczyk, N. E., Crutzen, R., Bolman, C., Muris, J. W. M., de Vries, H. (2013). Influence of delivery strategy on message-processing mechanisms and future adherence to a Dutch computer-tailored smoking cessation intervention. Journal of Medical Internet Research, 15, e28.

Stanczyk, N. E., Bolman, C., Smit, E. S., Candel, M. J. J. M., Muris, J. M. W., de Vries, H. (2013). How to encourage smokers to participate in web-based computer-tailored smoking cessation programs: a comparison of different recruitment strategies. Health Education Research, cyt104v1-cyt104.

Stanczyk, N. E., Bolman, C., Candel, M. J. J. M., van Adrichem, M. J. G., Muris, J. W. M., de Vries, H. (2014). Comparison of text and video computer-tailored interventions for smoking cessation: Randomized controlled trial. Journal of Medical Internet Research, 16, e69. 


\section{Other publications}

Guerrieri, R., Stanczyk, N. E., Nederkoorn, C., Jansen, A. (2012). Reward-sensitive women overeat in a varied food environment, but only when hungry. Eating Behaviour, 13, 31720.

Schulz, D. N., Smit, E. S., Stanczyk, N. E., Kremers, S. P. J., de Vries, H. \& Evers, S. M. A. A. (2014). Economic evaluation of a web-based tailored lifestyle intervention for adults: findings regarding cost-effectiveness and cost-utility from a randomized controlled trial. Journal of Medical Internet Research, 16, e91. 
DANKWOORD

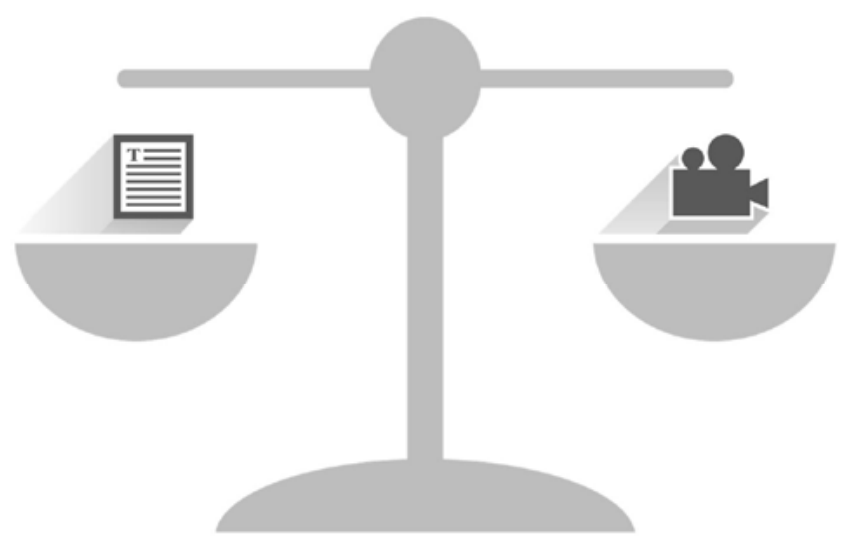



Graag wil ik als laatste een aantal mensen bedanken die op welke wijze dan ook hebben bijgedragen aan het tot stand komen van dit proefschrift.

Hein, Catherine en Jean, ik wil jullie bedanken voor de kans die jullie mij hebben gegeven om dit project uit te voeren. Ook wil ik jullie bedanken voor jullie expertise en wetenschappelijke begeleiding de afgelopen jaren. „The only source of knowledge is experience.” (Albert Einstein)

Mijn collega's wil ik graag bedanken voor de prettige samenwerking, het uitwisselen van kennis, ervaring, voor alle hulp, discussies en gezellige momenten op de afdeling.

"Success consists of going from failure to failure without loss of enthusiasm." (Winston Churchill)

Paul en Danou, fijn dat jullie mijn paranimfen willen zijn. Bedankt voor jullie steun en alle leuke momenten samen. Jullie humor en optimisme waren een goed tegenwicht voor alle tegenslagen de afgelopen jaren. „De beste manier om een probleem op te lossen, is de humor ervan te ontdekken." (Arthur Clarke)

Lieve familie en vrienden, dank voor jullie betrokkenheid, alle bemoedigende woorden en de vele gezellige momenten samen. „Kein Weg ist lang mit einem Freund an deiner Seite. "(Japans gezegde)

Liebe Mama, lieber Papa, liebe Oma's, ein kleines Wort „DANKE“ findet zu Euch, weil große Worte für alles was ich sagen möchte, viel zu klein sind.

Luc, jij compenseerde voor alle dipjes en stress die promoveren soms met zich meebracht. Bedankt voor je geduld, steun en dat je iedere dag weer met me oefent om te genieten van het NU. „Lebe den guten Augenblick, genieße das schöne Gefühl, vertage das Lebenswerte nie auf morgen. Morgen kommt zu spät." (Hans Kruppa)

Veel dank, Nicola 


